University of Louisville

ThinkIR: The University of Louisville's Institutional Repository

$12-2004$

\title{
The interrelationships of parent rural values, parent religiosity, parent involvement, and student outcomes in a small, southern, rural middle school.
}

E. Carolyn Tucker 1949-

University of Louisville

Follow this and additional works at: https://ir.library.louisville.edu/etd

\section{Recommended Citation}

Tucker, E. Carolyn 1949-, "The interrelationships of parent rural values, parent religiosity, parent involvement, and student outcomes in a small, southern, rural middle school." (2004). Electronic Theses and Dissertations. Paper 1464.

https://doi.org/10.18297/etd/1464

This Doctoral Dissertation is brought to you for free and open access by ThinkIR: The University of Louisville's Institutional Repository. It has been accepted for inclusion in Electronic Theses and Dissertations by an authorized administrator of ThinkIR: The University of Louisville's Institutional Repository. This title appears here courtesy of the author, who has retained all other copyrights. For more information, please contact thinkir@louisville.edu. 
IN A SMALL, SOUTHERN, RURAL MIDDLE SCHOOL

\title{
By
}

\author{
E Carolyn Tucker
}

B. A., Murray State University, 1972

M. A., Murray State University, 1980

\author{
A Dissertation \\ Submitted to the Faculty of the \\ Graduate School of the University of Louisville \\ In Partial Fulfillment of the Requirements \\ For the Degree of \\ Doctor of Philosophy \\ Department of Educational Foundations \\ University of Louisville \\ Louisville, Kentucky
}

December 16, 2004 
Copyright 2004 by E Carolyn Tucker

All rights reserved 
THE INTERRELATIONSHIPS OF RURAL VALUES, PARENT RELIGIOSITY, PARENT INVOLVEMENT, AND STUDENT OUTCOMES IN A SMALL, SOUTHERN, RURAL MIDDLE SCHOOL IN WESTERN KENTUCKY

$$
\text { By }
$$

E. Carolyn Tucker

B.S., Murray State University, 1972

M.A., Murray State University, 1980

A Dissertation Approved on

November 29, 2004

By the following Dissertation Committee:

Dissertation Director 


\section{DEDICATION}

This dissertation is dedicated

to my husband

Larry Allen Tucker,

Who learned to love, protect, and honor

All of my selves and gave us reason;

to my daughters,

Tari and Tami,

Who have no idea how much I love them;

and to my Mima,

Who always believed in me. 


\section{ACKNOWLEDGMENTS}

The author wishes to acknowledge several people for their contributions to the completion of this document. First is Dr. Stephen K. Miller at the University of Louisville, who, after enduring long hours and several visits to "the farm," has become a member of the extended Tucker family. He supplied answers to questions the author did know enough to ask. He went above and beyond the "call of duty" to help finish the cathartic, arduous tasks. And, most important, he would not let the author give up.

The other members of the dissertation committee, who were so patient, kind, and supportive throughout the "birthing" of this body of work, are acknowledged for their expertise and guidance. Dr. Dwight Cline and Dr. Christopher Wagner at Western Kentucky University, Dr. Darren Sherkat at Southern Illinois University, and Dr. Thomas Rieo at the University of Louisville were invaluable. In addition, Dr. Mary O'Phelan at Western Kentucky University and Dr. Beverly Moore at the University of Louisville offered statistical insight that escaped this numerically challenged researcher.

Because of the physical disability that struck half-way into the process, friends were called upon to taxi the author six-hour round trips back and forth to campus. Their help must be acknowledged. To Marilyn Clark, to Wilma Branson, and to Eaglewoman is offered heartfelt appreciation.

Finally, in memoriam, Dr. H. Richard Herrington, II, provided inspiration and challenge to reach always upward. 


\begin{abstract}
THE INTERRELATIONSHIPS OF PARENT RURAL VALUES, PARENT RELIGIOSITY, PARENT INVOLVEMENT, AND STUDENT OUTCOMES

IN A SMALL, SOUTHERN, RURAL MIDDLE SCHOOL

E Carolyn Tucker

December 16, 2004
\end{abstract}

This dissertation is a case study of 109 students and their parents from a small rural, middle school community in Western Kentucky. It seeks to determine the interrelationships between conservative Protestant Parent Values (Parent Rural Values and Parent Religiosity), Parental Involvement, and Student Outcomes. For the mixedmethods study, mail-administered Parent and Student Surveys provide quantitative data. Interviews, document analysis, and participant-observer field notes are utilized to supplement statistical findings. Ten Research Questions reflect Demographic Factors (Socioeconomic Status, Family Structure, and Specially Designed Instruction), Independent Variables (Parent Values--Rural Values and Parent Religiosity), Mediating Factor (Parent Involvement), and the Dependent Variable (Student Outcome). Rural Values constructs include Anti-Intellectualism, Conservatism/Traditionalism, Ethnocentrism, Good Old Boy, and Stewardship of Earth. Religiosity constructs are Belief, Ritual, Experience, Primary Affiliation, and Affiliation Bond. Parent Involvement constructs are Belief and Action. GPA measures Student Outcomes. 
Quantitative results include descriptive statistics reported for a small $N(38)$. Psychometric analyses reduce the number of variables because of the small $N$ and optimal scales yield Parent and Student Combined responses for nine of twelve scales. The separate constructs are then combined to produce a final set of scales for regressions: a reduced set of five Rural Values, one composite Parent Religiosity variable, and one Parent Involvement variable. Regressions reveal that only SDI and Anti-Intellectualism influence GPA. The small $N$ and possible biased sample (restricted range due to higher SES respondents) may be related to the fact that more variables are not significant.

Qualitative data provide supplemental detail establishing rich texture and a sense of a "living laboratory." Previously unexamined relationships between constructs such as Anti-Intellectualism, Stewardship of Earth, and Good Old Boy provide intriguing descriptions. In addition, this exploratory case study of the overlapping spheres of Parent Religiosity, Parent Involvement, and Rural Values offers insights into previously unrelated areas. 
TABLE OF CONTENTS

PAGE

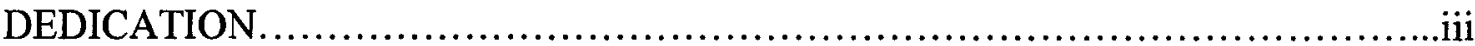

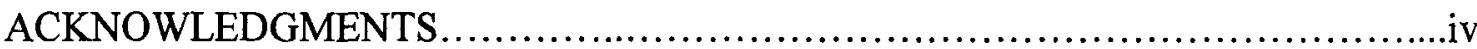

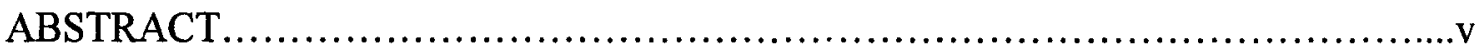

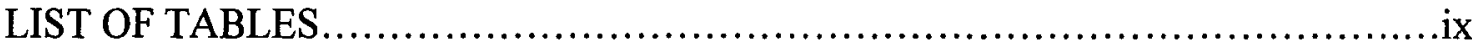

CHAPTER

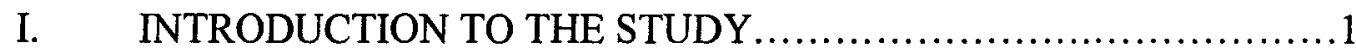

The Problem Defined.................................................8

The Setting...................................................

Significance of the Study ......................................25

Limitations of the Study......................................26

II. REVIEW OF RELATED LITERATURE..............................29

Parent Involvement...........................................30

Religion.................................................... 34

Religion and Secular Education...................................43

Rural Values...................................................57

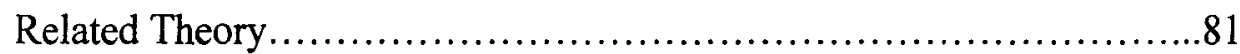

III. METHODOLOGY........................................... 98

Sources of Data..................................................99

Procedures..................................................... 105

Instrumentation and Variables.................................... 112 
Independent Variables.........................................115

Dependent Variable.............................................133

Supplemental Qualitative Data..................................... 135

Validation Process................................................141

Analysis of Data..................................................

Human Subjects Review Board......................................153

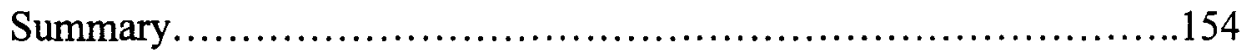

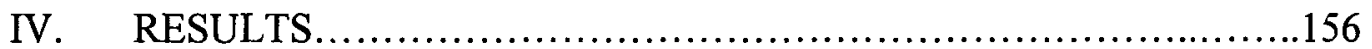

Descriptive Statistics................................................. 157

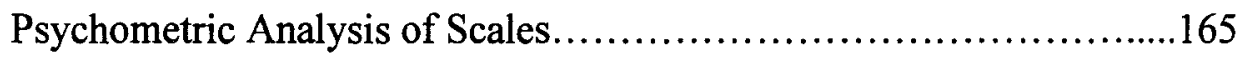

Research Questions...........................................185

Qualitative Analysis...........................................205

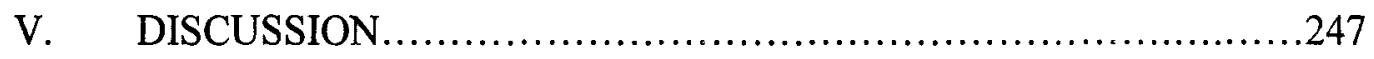

The Study in Brief................................................248

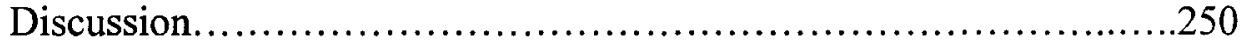

Recommendations.................................................285

Conclusion.......................................................290

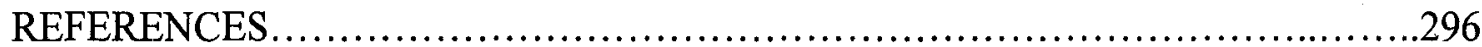

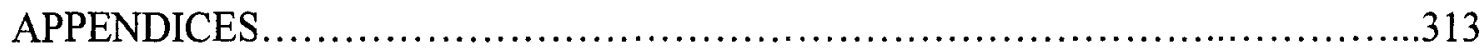

CURRICULUM VITAE ............................................................ 410 


\section{LIST OF TABLES}

TABLE

PAGE

1. Characteristics of Church as Primary Affiliation Group Versus Church as Secondary Affiliation Group...............................................93

2. Type of Multiple Regression Analysis across Variable Type by Research Question.

3. Descriptive Statistics for Family Structure..................................159

4. Descriptive Statistics for Socioeconomic Status Factors.....................160

5. Summary Descriptive Statistics for Rural Values...........................162

6. Descriptive Statistics for Parent Religiosity...............................164

7. Descriptive Statistics for Parent Involvement...................................

8. Initial Coefficient Alphas for Parent Values and Parent Involvement

Constructs.

9. Optimal Coefficient Alphas for Rural Values Constructs with Item Deleted....173

10. Optimal Derived Scales Based on Reliability Analysis......................174

11. Final Data Reduction Scales................................................176

12. Inter-Correlation Statistics for Optimum Parent Values and Parent Involvement Scales

13. Correlation Statistics for Parent Values (Rural Values and Parent Religiosity) and Parent Involvement Based on Optimal Coefficient Alpha....................180

14. Pearson r Correlation Matrix of Variables Utilized in Multiple Regressions.....186

15. Regression of GPA on Demographic Control Variables 188 
16. Regression of Social Conservatism/Traditionalism on Demographic

Control Variables.

17. Regression of Ethnocentricity on Demographic Control Variables.

18. Regression of Stewardship of Earth on Demographic Control Variables.

19. Regression of Good Old Boy on Demographic Control Variables

20. Regression of Parent Religiosity on Demographic Control Variables

21. Regression of Parent Involvement on Demographic Factors

22. Regression of Student Outcomes on Parent Values (Rural Values and Parent Religiosity)

23. Regression of Parent Involvement on Parent Values (Rural Values and Parent Religiosity)

24. Regression of Student Outcomes on Parent Values while Controlling for Demographic Factors.

T1 Reliability Analysis for Independent Variable: Rural Values-AntiIntellectualism.

T2 Reliability Analysis for Independent Variable: Rural Values-Social Conservatism/Traditionalism.

T3 Reliability Analysis for Independent Variable: Rural Values-Ethnocentricity..367

T4 Reliability Analysis for Independent Variable: Rural Values-Stewardship of Earth .368

T5 Reliability Analysis for Independent Variable: Rural Values-Good Old Boy...369

T6 Reliability Analysis for Independent Variable: Parent Religiosity-Belief.......370

T7 Reliability Analysis for Independent Variable: Parent Religiosity-Ritual.......372

T8 Reliability Analysis for Independent Variable: Parent ReligiosityExperience

T9 Reliability Analysis for Independent Variable: Parent Religiosity-Primary Affiliation 
T10 Reliability Analysis for Independent Variable: Parent ReligiosityAffiliation Bond.

T11 Reliability Analysis for Independent Variable: Parent Involvement-Belief......382

T12 Reliability Analysis for Independent Variable: Parent Involvement-Action...383

T13 Secondary Reliability Analysis for Independent Variable: Rural ValuesAnti-Intellectualism.

T14 Secondary Reliability Analysis for Independent Variable: Rural ValuesEthnocentricity....

T15 Secondary Reliability Analysis for Independent Variable: Rural ValuesStewardship of Earth.

T16 Reliability Analysis for Overall Parent Values and Parent Involvement Constructs. 


\section{CHAPTER I \\ INTRODUCTION TO THE STUDY}

John Taylor Gatto (1992), 1991 New York State's Teacher of the Year, issued a scathing indictment of schools in the United States, claiming that the public school is a "vampire network." He charged that because it "tears off huge chunks of time and energy needed for building community and family," it "needs to have a stake driven through its heart and be nailed into its coffin.... [It] has wounded us seriously in our ability to form families and communities, by bleeding away time we need with our children and our children need with us" (p. 57).

Not all critics of public education would agree, that, "schools are already a major cause of weak families and weak communities" which "separate parents and children from vital interaction with each other" (Gatto, 1992, p. 75). Few, however, would argue that parents are not as involved in their children's education as they should be, especially as children leave the elementary school and enter junior and senior high school.

On the other hand, if, as Gatto (1992) suggests, schools do serve as a malevolent force separating children from their parents, and if, as others claim (Epstein, 1995; Fullan 1991; O’Neil, 1991; Steinberg, 1996), parents must be involved in schools for our children to gain as much as possible from their educational opportunities, then it seems imperative to consider all possible causes for the great divide. Ultimately, awareness of causes for lack of parent involvement in schools could lead to solutions to the problem. 
Researchers have suggested and studied many possible reasons for lack of parental involvement in their children's education. For example, Epstein (1995) and Steinberg (1996) contend that "disengagement" of parents is a cause. Rosow (1991) believes parent illiteracy plays a part. Kelly and Pope (1995) identify conflicting schedules as part of the problem, and Barber and Patin (1997) explain that uninviting schools discourage parental involvement. Many teachers agree (D. Cohen, 1995): collaboration with parents takes up time--time that is unpaid, so it is rarely worth the extra effort. In addition, teachers often live in other communities and choose to maintain emotional distance for survival. Administrators, too, are often wary of non-educators entering their schools and they communicate those reservations to parents. Admittedly, all of these elements have been found to generate differing degrees of interference between schools and parents.

But in the conservative, rural South, another element might exert an even stronger wedge between schools and parents. Considering rural values, conservative/fundamentalist tendencies, and religious orthodoxy of the stereotypical rural South (Hofstadter, 1963; Seal \& Harmon, 1995; Sherkat, 1998), religiosity (the degree of a person's religious belief and practice) could be a factor deserving of attention as it pertains to parent involvement. A study by Peavie (1999) suggests that both religiosity and parental separation from children are factors in the fast-growing home school movement--especially in the rural conservative South. Peavie's results are supported by Philip Cohen (1995), who reported that the overwhelming majority of home-schooling parents are still devout Christians who seek control over the curriculum and values influencing their children" (p. 6). Sherkat (1998) also found that "both educational 
attainment and college-track curricula reduce faith in the Bible" (p. 1103), leading religious conservatives to complain that "secular education undermines faith in the Bible" (p. 1108). The University of New York's Graduate Center found that, as opposed to more liberal denominations, "Pentecostal and Baptist women attended college less often than non-religious women" ("Religious," 1995, p. 7).

Remarkably, however, few studies have been found to investigate seriously the impact of religiosity on parental school involvement in small, rural, Southern schools. Many studies of religiosity have, instead, concentrated on differences between Catholics and Protestants (Stark \& Glock, 1968).

\section{Background}

Schools in the United States are facing some of the most intense challenges since the beginning of public education and the establishment of the church-supported schools in colonial times (Fullan, 1991; Gregg, 1993; Hofstadter, 1963). "The American public school system was invented at a time when it seemed that the purpose of education was, or should be, to promote republican/Protestant morality and civic literacy ... [for] rural agrarian ... white Anglo-Saxons" (Schlechty, 1990, p. 5). From the earliest periods of American history, schools were established so students could learn to read the Bible. Classes were often located in the local church; teachers were often clergymen; texts were often religious in nature; and teaching centered on Bible truths and Christian doctrines. Even as late as the 1940s, rural schools allowed for instruction of Sunday school curriculum (Theobald \& Nachtigal, 1995). But many children were excluded from education under this system (Center on National Education Policy, 1996). "African Americans, Native Americans, many girls, and many poor white children who did not 
belong to a church" (p. 2) or children who lived in rural areas were not able to acquire an education. The supporters of public education have historically contended that education would help improve social conditions, decreasing crimes and violence and improving the possibilities for individual happiness and success, and by the 1850 s, free public schooling was available to most European-Americans.

Times have changed. Education experts tell us that in order to prepare our students to function successfully in today's world, our schools must address demands that were unfamiliar to citizens of the colonial period (Kentucky Board of Education, 1995; Kentucky Department of Education, 1993; Sizer, 1992). For example, according to demographic research (Crouch, 1995), Kentuckians, one of the least educated work forces in the U.S., must now face job changes for which they are not prepared: foreign employers, additional education requirements, short-term jobs, and a demand for continuously up-graded skills (G. Church, 1993). At the same time, Kentucky is evidencing a smaller work force, a larger educationally at-risk population, a smaller tax base, a decreasing economic base, "smarter" jobs, increasing dropout rates, and an inverted pyramid of workers/nonworkers-taxpayers/taxsupported (Crouch).

The Center on National Education Policy (CNEP) (Kober, 1996) reported, "Half of the people in prison in 1992 were high school dropouts" (p. 13). The CNEP also cited an estimate indicating “one murder, on average, costs society $\$ 2.7$ million" (p. 13). Additional statistics given by the CNEP underscore the continued need for public education: "People with more education have higher earnings. In 1993 male high school dropouts earned just two-thirds as much as their counterparts who graduated from high school but were not enrolled in college.... [And] high school dropouts are much more 
likely to be unemployed or on welfare" (p. 16).

In 1987, McLaughlin and Shields reported shocking statistics of school children: fourteen percent were illegitimate, forty percent lived in single-parent homes, thirty percent came home to empty houses, twenty percent lived in poverty, fifteen percent spoke non-English native language, fifteen percent had mental or physical handicaps, and ten percent had poorly educated parents.

Indeed, the demands of today's world are in stark contrast to the demands of colonial America. Being able to read the Bible and to do simple math will no longer suffice (DeYoung \& Lawrence, 1995). Moreover, the rural foundations of colonial America have shifted; now we are primarily a technological, industrialized society (Howley \& Howley, 1995). While before 1940, rural education valued "place" (or the community and its resources), and everything connected in some way to the "local," currently, for the most part, the valuing of "place" or "local" is considered primitive (Theobald \& Natchtigal, 1995). Emphasis is on learning to live in a global community-without much regard to rural values or place (DeYoung \& Lawrence, 1995; Seal \& Harmon, 1995). Often, entire issues of educational journals are devoted to global education and/or multiculturalism (e.g., Collins, Czarra, \& Smith, 1998). In contrast, only small publications by small groups offer consistent studies in the value of place or "rural-ness" (e.g., National Rural Education Association).

Responding to the assumptions that city life is better than farm life (DeYoung \& Lawrence, 1995), that city dwellers are smarter than country folk (Creed \& Ching, 1997; Fox, 1997), that urban culture is richer than rural culture (Theobald \& Nachtigal, 1995), and that industrial opportunities are much greater than agrarian (Herzog \& Pittman, 
1995), schools have been transformed from Bible-teaching, prayer directing, Christianvaluing, agrarian-preparing entities. They now seem to embrace the factory model of education (Sher, 1995; Theobald \& Nachtigal, 1995) that Elwood Cubberley claimed would "provide" children with the "necessary" skills for finding work in the larger, more culturally "advantaged" city. Even schools in the deepest, rural South teach subjects and content devoid of connections to rusticity or place (DeYoung \& Lawrence, 1995; Herzog \& Pittman, 1995; Seal \& Harmon, 1995).

At the same time, schools nationwide are seen as declining in the areas of student achievement, classroom control, moral education, discipline, expectations, and work preparation (Steinberg, 1996). According to experts, students are exhibiting a sense of being "adrift" due to many factors. Those factors may include divorce, low birth weights, poverty, suicide, child abuse, neglect, malnutrition, free sex, poor medical care, spiritual poverty, latch key families, loss of social capital, anti-achievement identities, and disengaged parents (Epstein, 1995; Gregg 1993; O’Neil, 1991; Steinberg).

According to L. Scott Miller (1995), "children from high-SES families, as measured by education and family income, do much better in school than low-SES children" because of the differences "in education-relevant family resources" available to each group (p. 337). "Cultural dissimilarities also account in part for the diversity of parenting strategies and parent-school relations associated with variations in student achievement patterns" (p. 338). Education-relevant family resources include an assortment of items and opportunities offered by the home environment: a computer, access to books and other print material, a home study area, study supplies, educational toys, trips to the zoo and museum, caretaker or parent tutoring, and active parent 
involvement in school. It is true, too, that "educational advancement [is] an intergenerational process ... dependent upon the quality of the education-relevant opportunity structure over a long period of time" (p. 339). The more educated people within the multi-generational history of the child, the longer the education-relevant resources have been available, the more positive the impact. Educators must also recognize, however, that the inverse is also true. If a child comes from a family where few individuals have been educated, if he or she has had few education-relevant resources available, if his or her education has been a family value for only one generation, then the child will be less successful in school.

Furthermore, anti-intellectualism has been noted in many American sub-cultures. For example, some minority children have received much less education-relevant resources, but they have also experienced an active education-repulsive pressure (cf. Ogbu, 1992). Anti-intellectualism was identified by Richard Hofstadter (1963) as a powerful force shaping American attitudes toward education as early as the 1950s. For example, the term "egghead" (p. 9) was used during the presidential campaign of 1952 to describe intellectuals--referred to as individuals who were extremely remote from the feelings of the common people. According to Hofstadter, the evangelist Billy Graham accused intellectuals of substituting reason, science, humanism, materialism, mind culture, and Freudian thinking for moral standards taught in the Bible. And schools were often chastised for having a too rigid system of education that emphasized academics instead of "good citizenship, occupational competence, successful family life, selfrealization in ethical, moral, aesthetic and spiritual dimensions" (p. 16).

Moreover, Southern, rural students face additional challenges to educational 
attainment. Since the early nineteenth century, the goal of the common man has been seen as the attempt to "build a society that would show how much could be done without literature and learning" (Hofstadter, 1963, p. 51). Given momentum by Jacksonian democracy, this goal was embraced most solidly by rural America. Particularly in the South, anti-intellectualism was affirmed as an element of egalitarianism. As a lasting reminder of this anti-intellectualism, today's rural students are still commonly stereotyped and tracked as non-intellectuals (DeYoung \& Lawrence, 1995). Even if students are intellectually gifted, they are frequently embarrassed and cajoled by their peers into embracing anti-intellectual stances or identities (Creed \& Ching, 1997; Fox, 1997; Herzog \& Pittman, 1995). At the same time, rural students are encouraged by the educational community to abandon the family farm or homestead for a better life in the city where the "smarter people are" (DeYoung \& Lawrence, 1995; Herzog \& Pittman, 1995).

The institutions that once supported Bible teaching, required prayer in schools, and honored agrarian careers now prohibit the religious connections, ridicule the agrarian choices, and threaten the conservative/fundamental values of a typical southern rural family (DeYoung \& Lawrence, 1995). Sadly, the professionals who have the task of educating the rural students have received little or no training to address the specific needs of those students (Herzog \& Pittman, 1995). It is not surprising that modern reformers "loathe the industrial/factory model of schooling that has come to dominate American K-12 education" (Sher, 1995, p. 143).

\section{The Problem Defined}

Because education must place emphasis on teaching students to survive in a 
global economy, the valuing of the "local," or "place," and all of its culture, seems to have been neglected (DeYoung \& Lawrence, 1995; Herzog \& Pittman, 1995). However, to farmers or people employed in farm-related jobs, who have willingly accepted stewardship of the earth (Verhoog, 1995; Verkleij, 1995; Witte, 1995; Zweers, 1995) (as well as the responsibility for producing all food resources), a sense of place is one of the most important values (Howley \& Howley, 1995). In fact, their culture is built on the "love of the land"--their place (Verkleij, 1995). To help educators realize the need for valuing place in a rural setting (and it would follow, that in order for rural students to attain as much educationally as they can), it would be logical to assume that rural parents must be re-engaged in their children's educational processes and in connecting education to rural life. However, educators must develop an awareness of the reasons parents are not involved before those reasons can be addressed for solutions.

The problems experienced by urban educators, as the educators try to involve parents in schools, are problems that are realized by rural educators, also. In addition, rural educators contend with a little recognized source of potential interference:

Conservative Christian religiosity exerts powerful, even if not recognized, pressure on the actions of people who live in the rural south (Seal \& Harmon, 1995).

An ordered look at the topic in question reveals a need for research into the area of conservative religion and its impact on student achievement as mediated by Parental Involvement (PI):

1. PI is mandatory for students to benefit as much as possible from their education (Epstein, 1995).

2. Many programs exist to promote PI, but, at the same time, many barriers serve to impede the involvement of parents on different levels (Steinberg, 1996). 
3. PI decreases significantly as children enter middle school (Barber \& Patin, 1997).

4. Although causes of limited PI have been identified, few studies have examined how conservative religiosity relates to PI, and, ultimately, to student outcomes.

Developing a general awareness of the differences and degrees of impact exerted by varying levels of religiosity on PI and middle school might provide an understanding of additional barriers to or supports for PI. With that added to the element of conservative rural values, a significant relationship to PI (positive or negative) could be identified. With awareness and understanding, ways to mitigate negative relationships, or ways to emphasize positive relationships through linkage with place and school, might be suggested. The results of a study could be used to improve motivation for PI, and, consequently, increase levels of PI. In turn, this could increase the potential for improved student outcomes.

\section{Purpose of the Study}

This study seeks to investigate the relationship of parent religiosity (PR) to parental involvement (PI) in a small, secular, rural, Kentucky middle school. It will be an exploratory case study of mixed design (using qualitative and quantitative measures) to establish a baseline description of "what exists" in the Dixon Elementary/Middle School. Although a case study is limited in scope, it is sometimes possible to examine larger social forces through the lens of a specific site. In this regard, the current study can be a window to examine the overlapping relationships of rural values, conservative parent religiosity, parent involvement in rural southern middle schools, and student outcomes. Through surveys that look at parental rural values and religiosity, kinds and quantities of parental involvement, and student perspectives, and through qualitative descriptions of 
the rural environment and the rural culture, a clearer picture can be drawn of how these elements influence student grades by investigating their independent and overlapping spheres of relationship.

The topic to be addressed is, how do conservative Christian religiosity, rural values, and parental involvement (PI) influence the rural Southern school? Studies have shown that PI decreases as children advance into middle school (Barber \& Patin, 1997), but few studies have considered religiosity as a possible element in the change of PI. Thus, the central Research Question can be refined as: How do conservative Christian parent religiosity $(\mathrm{PR})$ and southern parent rural values $(\mathrm{RV})$, as mediated by parental involvement (PI), and controlling for demographics, relate to student outcomes (SO) in a southern rural middle school?

\section{Research Questions}

Researchers must recognize the unique characterizations of rural culture at the outset of any study of rural values. Assumptions are widely accepted characterizations that may be held as valid for a population under study.

\section{Assumptions}

Given the Central Research Question, there are four assumptions that can be made about the rural culture concepts underlying this study:

1. Rural cultures differ from other cultures in values and educational opportunities.

2. Rural culture tends to be conservative.

3. Rural culture is perceived to de-value education.

4. The Rural South has a high concentration of conservative religions and members of conservative religions. 
The primary research question explores the factors considered in the assumptions: conservative Christian parent religiosity (PR), parent rural values (RV), and parental involvement (PI). These factors are examined in the context of demographics for their effects on Student Outcomes (SO), all as situated in a southern rural middle school. The exploratory case study addresses three types of independent variables--demographic controls, alterable parent values (rural and religiosity), and the mediating factor of parent involvement; these will be analyzed for their relationship to the dependent variable of SO. (The different types of variables are illustrated in the theoretical model in Figure 1.) 


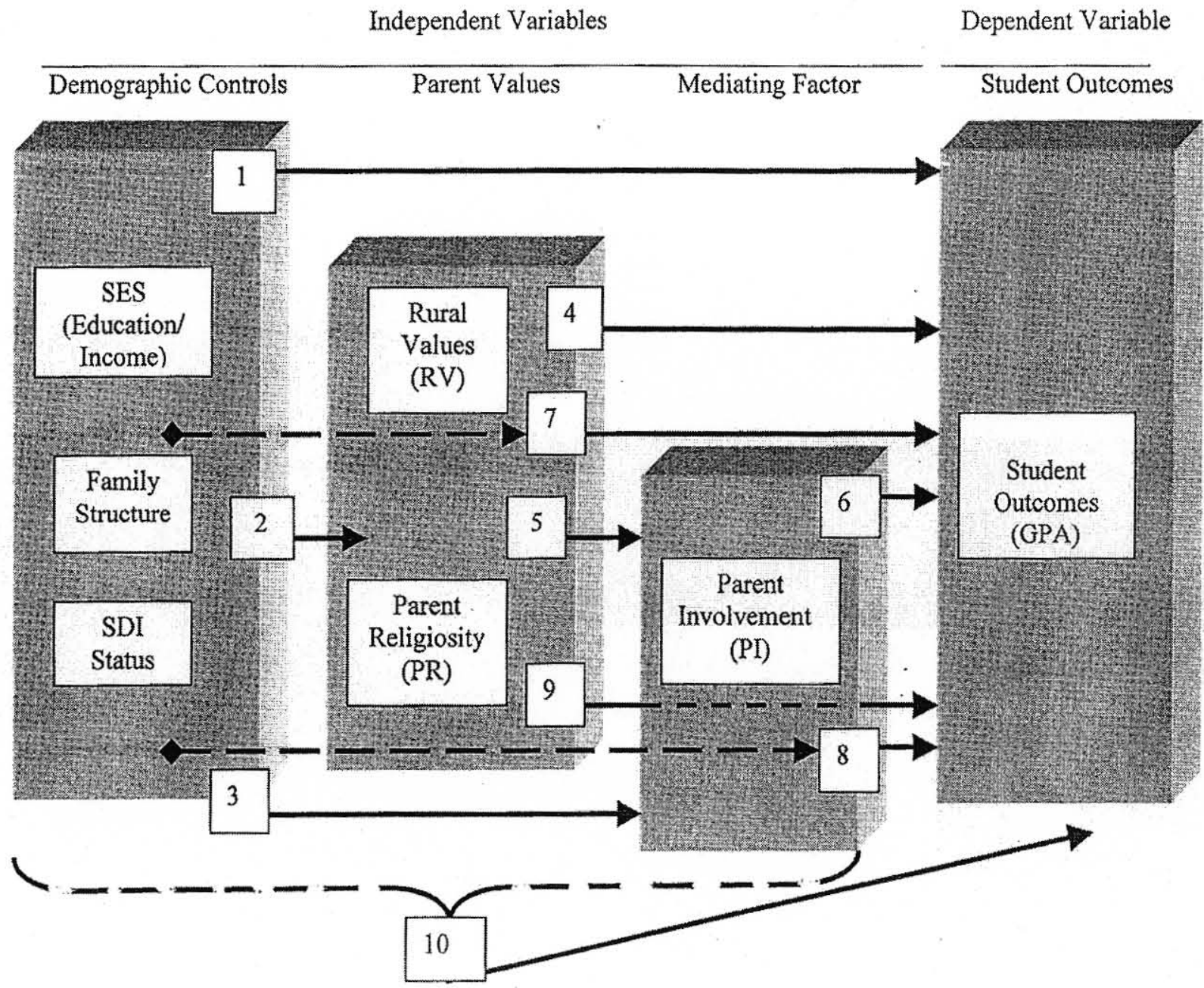

Figure 1. The relationship of Parental Rural Values and Parent Religiosity to Student Outcomes as mediated by Parent Involvement and controlled for Demographics. 
Figure 1 illustrates the independent variables of parent rural values and parent religiosity as they relate to parent involvement and student outcomes. Also illustrated are the independent variables of demographic controls: parent socioeconomic status (SES), family structure (FS), and student special education--specially designed instruction (SDI) status. Arrows provide indications of possible relationship between each of the variables and between their overlapping and/or mediating spheres of relationship. The arrows are numbered to correspond to the following research questions:

1. How do Demographic factors relate to Student Outcomes?

2. How do Demographic factors relate to Parent Values:

a. Rural Values?

b. Parent Religiosity?

3. How do Demographic factors relate to Parent Involvement?

4. How do Parent Values:

a. Rural Values

b. Parent Religiosity relate to Student Outcomes?

5. How do Parent Values:

a. Rural Values

b. Parent Religiosity

relate to Parent Involvement?

6. How does Parent Involvement relate to Student Outcomes?

7. How do Parent Values:

a. Rural Values

b. Parent Religiosity relate to Student Outcomes, controlling for Demographic factors?

8. How does Parent Involvement relate to Student Outcomes, controlling for Demographic factors?

9. How do Parent Values:

a. Rural Values

b. Parent Religiosity relate to Student Outcomes, as mediated by Parent Involvement? 
10. How do Parent Values:

a. Rural Values

b. Parent Religiosity

relate to Student Outcomes, controlling for Demographic factors, as mediated by Parent Involvement?

\section{The Setting}

In order to have an accurate picture of the situation under investigation, a thorough examination of the specific school environment to be studied is presented.

Dixon Elementary/Middle School is located in Dixon, a small rural community of about 4,000 residents, the centrally located county seat of Webster County in Western Kentucky. Most people in the community are employed in coal mining, in farming related jobs, in light manufacturing, or in the county school system. Some residents commute to Evansville, Indiana (about 50 miles away), to work in factories there. Most of the inhabitants belong to the upper-lower or the lower-middle economic level. Few minorities live in the community in spite of the influx of migrant workers for the chicken farms that have recently spread throughout the county.

Dixon's central commercial district consists primarily of a family hardware store, a small bank, the court house, a chiropractor's office, the post office, a flower shop, a gas station, the city offices, a Bible book store, a Human Resources office, a hometown lawyer's office, several empty buildings, and one stop light on Main Street (old Highway 41). Because it is a stereotypically lazy little town, it is not uncommon to see whitehaired men sitting on courthouse park benches watching traffic, playing checkers, or greeting passers-by year-round.

Houses of worship in Dixon include a General Baptist church, two Southern Baptist churches, and a Methodist church. Within two or three miles of downtown are a 
Church of Christ, a Freewill Baptist church, another General Baptist church, and another Southern Baptist church. Serving people within a few miles from Dixon are other nearby houses of worship: a Catholic church, another Church of Christ, several more Baptist and Methodist churches, a Jehovah's Witness Temple, a Christian church, Assembly of God churches, Pentecostal churches, a Church of God in Prophesy, Apostolic churches, Cumberland Presbyterian churches, Presbyterian churches, a Church of God in Christ, and several non-denominational churches. Many of theses churches are very small, and "new" churches have often been the result of splintering of existing churches due to disagreements on doctrine, social values, and family feuds (though most of the splinterings occurred some time ago).

The county is divided into a county school system and an independent city school system. Providence, the largest town in Webster County, and the town closest to Dixon, maintains two independent schools, Broadway Elementary and Providence High School. However, the schools are very small: Broadway enrolls about 350 students and Providence High School enrolls around 125 students. Many of the students in the city districts attend Dixon Elementary and Webster County High School on contracts: they are allowed to attend schools outside their district of residence tuition free on a reciprocal basis.

Just south of Dixon city limits lies the Webster County school campus which is made up of the high school football field and several connected buildings: Webster County High School, Webster County Annex, Kentucky Tech, and Dixon Elementary/Middle School (DEMS). (Other county elementary schools are located in Sebree, Slaughters, and Clay.) The basic description that follows is based on the 1999- 
2000 school year, although dates and years have been updated to the 2002-2003 school year where appropriate.

DEMS was built in the late sixties as one of the state's experiments with open school concept. It originally had few interior walls except for those around the restrooms, the office complex, the music room, and the media center office. The class areas were linked in a circular fashion around the restroom block, in such a way that a person standing in one corner of the building could communicate with someone standing in the farthest corner. Not long after moving into the building, however, teachers began to "manufacture" their own walls with bookshelves, filing cabinets, storage centers, etc. Subsequently, in many areas, walls were added between sections of the building and few areas remain without some walled spaces. There are currently five walled class sections: Early Primary (EP) is walled off from Late Primary (LP); fifth and sixth grades are walled off from seventh and eighth grades; and most of the special education teachers (Specially Designed Instruction or SDI) and the computer lab instructor have succeeded in having movable partitions installed around their areas.

As part of the Webster County School District, DEMS, for seven years, was under the leadership of a male superintendent reared in another rural Kentucky community. In July of 2000 , the superintendent's position was filled by a long-time resident of Webster County, who has served the county school system in a variety of roles: teacher, principal, assessment coordinator, and assistant superintendent. His wife is a teacher in the high school; his daughter (formerly a student at DEMS) is now a teacher at DEMS. Summer of 2002, an unemployed man (from the Covington, Kentucky area) was hired as superintendent. Other leadership is provided by a five-member school board (for the year 
2000 it was made up of a rural mail carrier, the wife of a local car salesman, a stay-athome mother, a vocational school teacher, and a farmer), a male principal, and a SiteBased Decision Making Council (SBDM).

The male principal of DEMS, who came to DEMS in 1997, was formerly an agriculture teacher at the high school where he gained a reputation for being anti-reform: he was outspoken in his resistance to KERA. He currently lives near Dixon with his wife (who substitute teaches at DEMS) and three daughters (who all graduated from DEMS). He farms tobacco and raises horses and hunting dogs. He is a life-long resident of Dixon and a graduate of DEMS (he and the author of this study have been schoolmates and acquaintances since 1954).

He and his family are active members in the Dixon Methodist Church: he and his wife both teach Sunday School classes and often participate in other church activities. His daughters are leaders in church groups and, as well, have led observable Christian dialogues and activities at school. They have been prominent leaders at DEMS in academics (all three girls were valedictorians of their classes) and athletics (basketball, track, cross-country, tennis).

The SBDM in 1999-2000 was made up of a pre-school teacher, an SDI teacher, a middle school teacher, and two stay-at-home mothers. The council has a conservative history, tending to serve as a rubber stamp to the principal's pre-conceived plans rather than as a truly working representation of the school community. Few ideas or proposals have had genesis within regularly scheduled SBDM sessions during the current principal's tenure.

The DEMS faculty has been very stable and changes in faculty placement take 
place rarely. Currently seven teachers have been at DEMS for more than twenty years; six have been at DEMS for more than fifteen years; and two are former DEMS students who have returned to teach. Much of the support staff reflects the same constancy.

Organizationally, the school structure is patterned much like the walled spaces. The Early Primary includes students in their first, second, and sometimes third year of school. The Late Primary students are generally in their second, third, and sometimes fourth year of school. A preschool room was added to an end of the building away from the EP in 2000. Students traveled to the EP for integrated work frequently during the week. However, beginning in the fall of 2002, the preschool room was closed due to lack of enrollment. Students are bussed to another county school, and the preschool room is undergoing transition into fulfillment of other needs. The "fourth grade" students were located in what was designed to be the "amphitheater" area of the school and the school "stage." They are considered "self-contained" in that they are instructed by the same teacher all day long, except for special classes. The "amphitheater" fourth-graders have an area of generous space for centers and group work, but the "stage" fourth-graders are confined to a space that is cramped and difficult to maneuver through. Fifth and sixth grade students are taught (as a departmentalized section) in the largest area of the building, near the old preschool room. They are located near the media center, the outside door, the office, and the restrooms.

DEMS has a student enrollment of approximately 420; about 109 of those students are in the seventh and eighth grades ( $97 \%$ European-American/white). The school was created to be a K-8 elementary, but with recent changes brought on by the Kentucky Education Reform Act (KERA) and emphasis on middle school education, the 
seventh and eighth grade sections refer to themselves, and function as, the middle school.

The two seventh and two eighth grade classes are located near the back of the building. Though enlarged by a new science lab, added at the same time the pre-school room was built, the area is space-limited and congested. Recently partitions were installed which cut the useable space even more. The classes are departmentalized into Language Arts, Social Studies, Math, and Science for four core hours of the day. Electives are offered for the remaining time.

Language Arts and Math are ability grouped. The teachers of those subject areas feel that more progress can be made by the more academically able by grouping them. Curricular differences resulted in the higher-level group receiving instruction in algebra and more writing opportunities in the Language Arts. Science and Social Studies are heterogeneous classes: groups are determined by balancing SDI, gifted, slower, academically able, "trouble-makers," and natural leaders in each class.

Each seventh and eighth grade student moves through class area to class area during the day to receive instruction in all four "core" subjects. Additionally, students may choose from "electives" that are offered during the first hour and last hour of each day. In order to go to any other part of the building (restroom, cafeteria, library, office, gymnasium, music room) students must travel through at least one additional class area, usually through two class areas and sometimes three depending on point of origin. Disruptions and distractions are frequent as students move from one section to another. Electives offered to middle school students have included band, chorus, performing arts, speech, health, first aid, advanced physical education, art, library, computer skills, and French. An apprentice program also utilizes students who choose to work during their 
elective periods for staff members in many parts of the building. Extra-curricular activities provided for middle school students have included academic teams, speech teams, math team, Beta club, performing arts, band, basketball, football, track, crosscountry, cheerleading, drill team, and 4-H.

Each grading period the middle school students are offered an incentive trip. If they "earn" the privilege (through good behavior, passing grades, working in class), they are permitted to go on the incentive trip: bowling, skating, movies, camps, museums, and other enrichment opportunities. Parents are allowed to accompany students on the incentive trips. In fact, the trip permission form always states: "Parents are encouraged to participate in our incentive trips."

The Language Arts teacher from 1994-2000 is now the school counselor. She lives in an adjoining county and drives sixty miles round-trip daily to work; she lived in Webster County for over fifteen years before moving away in 1997. She has taught at Dixon for five years and is an active Methodist who shares her Christian testimony with students during counseling sessions and during religious holiday activities (Thanksgiving and Christmas, for example). The Math teacher also lives in an adjoining county and commutes daily. He has taught Math at Dixon for twenty years, although Math is not his area of certification. He is certified in PE but was "grand fathered" into math. He is well known and remembered by his students for his frequent lectures encouraging students to become involved. He readily shares his Christian faith with his students. The Social Studies teacher has been moved into this position after having taught Science at DEMS for over twenty years. He is certified in Social Studies, but he had not taught it until fall of 2000. He has lived in Dixon for over twenty years and is an active member of the 
Church of Christ. For some students, he serves as a church activity leader. The Science teacher is in his third year as a middle school teacher, having been moved from the fifth and sixth grade area this year. He also serves as the middle school football coach and is open about his Christian faith.

The author of this study currently remains at home on disability. She has served as the Social Studies, Language Arts, Speech, Drama, Art, and French instructor. She is a life-long resident of the Dixon area and a teacher of more than twenty-two years for the Webster County system. As an active member of a local Baptist church, she has (in years past) served as Sunday School teacher, Bible Training leader, and Youth Choir leader to many DEMS students. When still teaching she was open about her Christian faith at school, but she also taught about other faith doctrines as part of the Social Studies curriculum: Hinduism, Buddhism, Confucianism, Taoism, Judaism, Islam, and ancient Greek, Roman, and Egyptian mythologies.

The final faculty member in the junior high area is a second-year teacher who lives in Clay, the nearest town to Dixon. She replaced the author of this study upon her disability leave. She is middle-aged and the mother of grown children. She openly speaks of her religion and "witnesses" to others around her. Her husband was at one time a lay minister of the Baptist faith.

Students who make up the student body of DEMS are drawn primarily from the Dixon community; however, several students commute from other districts, both inside and outside Webster County. Students graduating from DEMS usually move to Webster County High School, though some do transfer to the independent city school in a nearby town, Providence, or other schools in adjoining counties. During the 1999-2000 
academic year, there were between fifteen and twenty students who were officially part of the Dixon district who attended a nearby parochial school, Christian Heritage Academy. That school has since moved out of the district and no Dixon Elementary/Middle school students are known to attend CHA at this time; however, the students attending during the 1999-2000 school year are relevant to this study. Two middle school students, who had withdrawn to attend Christian Heritage Academy, returned to Dixon School for the 1999-2000 school year. Some students have withdrawn from Dixon to home school.

Parent involvement at Dixon is limited mostly to support of athletic teams. Though the PTO claims many members, only a very few are actively involved. Rumors circulated in 2000 warning that the PTO would be dissolved because no parents would serve as leaders. But no official report of plans for such dissolution was ever given to the staff. SBDM committees have only one or two parent members; on some committees there is no parent representative. The fall and spring regularly scheduled evening parentteacher conferences typically draw only ten to twenty visitors to the middle school section, as illustrated by the "Sign in Sheet" in Appendix A.

The School Report Card prepared by the SBDM Council (Dixon, 2000) for the 1999-2000 year (see Appendix B) supplies school academic statistics based on the Commonwealth Accountability Testing System (CATS) for the preceding year. The CATS also includes self-reported Parental Involvement (PI) scores. Such information provides a description of student outcomes, and some evidence as to the degree of PI at the school. The school uses the Kentucky Core Contents Test (KCCT) of the Commonwealth Accountability Testing System (CATS) to assess student progress and 
outcomes at grades 7 and 8 . The CATS also evaluates attendance rates and retention rates. Prior to CATS, Kentucky Instructional Results Information Systems (KIRIS) provided information of student achievement using a combination of tests and portfolio assessment. The Comprehensive Test for Basic Skills-5 (CTBS-5), a nationally-normed test, providing percentile scores of student basic skills that allow for comparison nationwide, is given at the sixth grade. However, no nationally-normed test was given to students at grades 7 and 8 during the years of study.

In 2000, DEMS students at grade 7 scored 62 (out of 140) in reading, 33 in science, and 27 in writing (state scores were $68,37,39$ ) on the CATS. At grade 8 , students scored 78 in math, 54 in social studies, 40 in arts and humanities, and 35 in practical living/vocational studies (state scores were $67,51,39,34$ ). Non-academic data were collected from 1997-1998 figures. They include average daily attendance, $94 \%$ (state, $94 \%$ ), retention rate, $0 \%$ (state, $3 \%$ ), and student dropout rate, $2 \%$ (state, $1 \%$ ). The School Report Card (Dixon, 2000) also indicates that $100 \%$ of the classes were taught by teachers with a major, minor, or equivalent in the subject, and $77 \%$ (state, $78 \%$ ) of teachers had a masters degree or higher. The average number of years of experience was 17 years (state, 13). Evidence of involvement by parents includes 120 enrolled in parent organizations, 200 hours of volunteer service, 5 who served on school council or its committees, 75 who voted in school council elections, and 225 students who had parents who attended at least one teacher conference during the year. Only $6 \%$ of the classrooms had "telephones able to access outside lines" (Dixon, 2000, p. 3); however, telephones have since been added to each classroom.

Student-to-teacher ratio is listed at 17:1 (state, 18.7:1), but the student-to- 
computer ratio is listed at 11:1 (state, 7:1). Per-student spending was said to be $\$ 4,338.59$ while the state figure was $\$ 6,498$. Because these figures were self-reported, there exists some doubt about the accuracy of the stated amounts. The school mission reads, "To meet the unique needs of every child with the assistance of parents in our community" (Dixon, 2000, p. 1).

\section{Significance of the Study}

There exists a great wealth of research on the types and benefits of PI (Epstein, 1995; Dornbusch, 1988; Fehrmann et al., 1987; Fullan, 1991; Henderson, 1987; Maxim \& Five, 1997; O’Neil, 1991; Smith \& Martin, 1997; Steinberg, 1996; Tennies, 1986; Useem, 1991). Organizations and programs have been established nation-wide to address the specific needs evidenced by middle school learners, and student achievement at all levels has been a focus of numerous studies for many decades. However, only recently, more consideration has been given to the element of rural values or sense of place in the development of schools within the rural areas of America (Little, 1998). DeYoung and Lawrence (1995) claim that the professional education community "has historically ignored questions of the social purpose of schooling in the U.S. when it comes to either rural or urban schools" (p. 112).

While religiosity has been studied as an important social force, few studies have investigated the impact of religiosity on secular education. One reason for the lack of research in the area appears to be lack of funding for such projects. Churches have conducted studies, but usually only those useful as they apply to their denominations (Stark \& Glock, 1968). Even as recent as the October 2000 Conference, out of approximately 170 presentations offered by the Society for the Scientific Study of 
Religion on Religious Research at their annual conference in Houston, Texas, only one or two were focused primarily on religion's role in secular education (Society, 2000). Another possible reason for the few large-scale research projects on sociology of religion is the protest from conservative religious groups who suspect such studies. In light of the apparent void, Ellison and Sherkat (1997) call for further research that determines the impact of religiosity on parenting: the effects on academic achievement outcomes for children.

Though conservative religious groups and influences have increased, in number as well as influence (e.g., the Religious Right, the Christian Coalition, Southern Baptist Convention, Focus on the Family, religion-based home schools) (Peshkin, 1986), few researchers have looked at the impact of religiosity within the secular school environment. Because of the relative lack of such research, religiosity is examined for its role in parents' school involvement, and, specific to this study, in Southern, conservative, rural schools.

\section{Limitations of the Study}

Because this is an exploratory study, many areas could be included in the investigation. The relationships of values, PI, and SO might vary at other levels within the same school. The different religious affiliations in other school communities might cause other relationships to appear. Generalization of data is impossible considering the case study methodology and small $N$. Minority differences might affect the outcomes of a similar study done elsewhere. The impact of parental involvement could easily extend into post-secondary education and well into adult career choices, but those areas are beyond the scope of this study. 
However, in order for the study to be an in-depth exploratory investigation of one school, and so that the study can remain "do-able" within the time constraints allowed, the study constitutes an exploratory case study of the middle school section of one small rural school in western Kentucky.

All conservative religious denominations, considered conservative Christian by experts in the field, are treated as a monolith because the questions deal only with conservative religiosity, not with particular denominational interpretations. Generalizing for denominations based on information gleaned from this study is not possible.

Denominations generally considered Conservative, however, can be further divided within this small community into three groups: Ultra-conservative (or "literalistic"), Conservative, or Moderately Conservative.

Minority impact is not considered separately in this study because the school in question has less than $1 \%$ non-European population at the middle school level. Though PI and PR could well affect minority populations, the minority population within this school community is not large enough to measure. In addition, the small number of minorities limits the generalization of results to broader populations.

Even though parental impact could extend into college and adulthood, that area of concern is beyond the scope of public education and of this study. Therefore, this study of a middle school section focuses only on the period of schooling that is still considered elementary, but, at the same time, is the area that evidences the greatest absence of PI, which, again, limits its generalizability.

Because this study is an exploratory case study and there is not a high survey response return (low $N$ ), that fact limits complete quantitative analysis. Testing the 
proposed theoretical model assumes the need for multiple regression, but multiple regression requires an adequate ratio of cases to Independent Variables. The $N$ in this study falls short of that ideal ratio. However, an exploratory case study is used for development of theory, not testing of a model. Consequently, the quantitative analyses used in this study are limited to examining sections of the proposed model as the data allow. These results inform theory building concerning the value of rural culture and its effect on schools. The full model can be further tested in subsequent research.

The study is further limited by incomplete return of surveys, by virtue of the selfreporting nature of the surveys and interviews, and by the possibility of unintentional bias injected by the researcher who is a Baptist and has been a twenty-year faculty member at the school. That potential for bias is addressed in several ways in Chapter III.

\section{Summary}

In summary, it can be said that there is a substantiated need for PI in education in order for students to achieve. It is also clear that there is not enough PI in most schools, especially from the middle school up, and that children and school systems suffer because parents are uninvolved. Many factors have been shown to relate negatively or positively to PI. However, in spite of the claims that America was founded primarily as a Christian nation, and that schools in America were begun by Christians to aid in religious education, few studies have focused specifically on the role or relationship of religiosity to PI. This study focuses on one aspect of that issue as reflected in the central research question: How do conservative Christian parent religiosity (PR) and southern parent rural values (RV), as mediated by parental involvement (PI), and controlling for demographics, relate to student outcomes (SO) in a southern rural middle school? 


\section{CHAPTER II}

\section{REVIEW OF RELATED LITERATURE}

If according to our Puritan heritage, America was founded to provide freedom for Christian Protestants to worship as they chose and, as an integral part of that freedom, to build schools in support of religious education, it is then natural to assume that religiosity has historically had a strong relationship to student outcomes and to parent involvement. How that relationship has changed over the years since our founding fathers stepped foot on Plymouth Rock has been the subject of few studies, but the ideal of fundamental Protestant values still provides a force of strong percussion, especially in the rural South.

Education has changed. No longer does it exist exclusively to teach future Christian citizens to read the Bible. No longer do schools need to prepare students merely to be able to defend rights and freedoms won at great cost. A few years of learning basic reading, writing, and math from the local schoolmaster, hired by the local church, will no longer suffice for today's youth. As American society has grown and evolved, so have the obstacles to education, and impediments to student achievement are more numerous and varied than ever before. According to Jones, cited in Roberts (1995), since 1960 "teenage suicides and homicide rates have more than tripled, [and] the current generation of children and youth is the first in our nation's history to be less well-off-psychologically, socially, economically and morally--than their parents were at the same age" (p. 204). At the same time, the need for a sound education has become more 
necessary (D. Cohen, 1995): as recently as 1970 there were five workers to every retiree; in 2030 the ratio is expected to be 2.6 to one ("Baby," 1996). According to many scholars (including Schlechty, 1990, and Haas \& Lambert, 1995), a team approach is needed to address such social and economic bankruptcy. In fact, some school restructuring specialists advocate that every stakeholder in the school community (anyone who has something to gain or lose by what happens) should be involved in the struggle to rise to the challenges (Schlechty). More emphatically, Mitroff (1983) goes so far as to say, "The state of an organization ... will be the result of the interaction of the behavior of all the organization's stakeholders from the beginning of its history up to a particular point in time" (p. 373).

In other words, the stakeholders must be motivated to become involved in order for any organization to operate at the optimum level; schools are no exception. Schlechty (1990) identifies the groups of stakeholders critical to the success of schools as teachers, teacher groups, administrators, parents, civic leaders, legislators, businesses, community organizations, and taxpayers.

What is known about the impact of these stakeholders on educational attainment in secular settings can be viewed through several categorical lenses of existing literature that are relevant to the current study: Parent Involvement, Religion, Religion and Secular Education, Rural Values, Related Theory, and Affiliation Theory.

\section{Parent Involvement}

Much research suggests that the most significant of stakeholders in the school community is the parent (or primary caretaker) (Clark, 1995; Cushman, 1993; Davies, 1996; Fullan, 1991). According to the Coleman Report (1988), the child's home 
environment accounts for as much as $65 \%$ to $85 \%$ of the variance in student achievement. Student achievement, though not the only area of improvement, is the primary area that realizes significant gains when parents become involved in their child's education (Epstein, 1995). The earlier Parent Involvement (PI) begins, the more ardent it is, the longer it lasts, the more significant the benefits are (Fullan, 1991). PI is beneficial in raising student achievement at all levels in many ways (Cushman, 1993; Dornbusch, 1988; Fehrmann et al., 1987; Henderson, 1987; Tennies, 1986; Useem, 1991). And it is crucial in order to "ensure the best possible learning environment for children" (Maxim \& Five, 1997, p. 1). The U.S. Secretary of Education, Richard Riley (1996), proclaims parents to be an "essential link in improving American education" (p. iii). In 1996, the U.S. Department of Education released a report that summarized thirty years of research in the area of PI. The report states that $90 \%$ of the difference in students from schools with high performance levels compared to schools with low performance levels could be explained by three factors: length of time watching television, absences from school, and the variety of reading materials at home. Obviously, all three elements are within the domain of PI.

The 25th Annual Phi Delta Kappa Gallup Poll of national public attitude toward public education (Elam, Rose, \& Gallup, 1993) revealed that in nearly every demographic group surveyed, $96 \%$ of the public thought parent involvement in children's education is very important. A PTA poll cited in the same report indicates that $95 \%$ of parents surveyed wanted written plans for PI because they felt it was "crucial to school success" (p. 149).

Although all forms of PI result in gains in student achievement, thirty years of 
study suggest that it is active PI that truly reaps the most benefits. Active PI may be defined as the participatory roles the parents play in the students' learning activities. Their roles may include working with the children at home on homework or projects, attending and supporting school activities, serving as assistants or tutors in the classroom, becoming learners themselves, and being realized as partners in their children's educational processes (Fullan, 1991; Meade, 1992).

Joyce Epstein (1987), in an article for Principal Leadership journal and in an interview for Educational Leadership (Brandt, 1989), identified five types of PI: (1) Parents are involved with their children at home so that they are mentally and physically healthy and strong and so that they have an environment that will support learning; (2) Parents are in contact with the school as often as possible to make sure they understand the school's programs and their child's progress in those programs; (3) Parents are involved actively in the school as tutors, classroom assistants, and coaches; (4) Parents are involved with the child's assignments, providing support and assistance at home; and, (5) Parents are involved in school groups that provide direction for the school and its mission. Epstein (1995) later adds a sixth dimension of PI--collaboration with community.

Active PI is evidenced most often at the primary levels, when children are still strongly bonded to parents, when parents are more protective of the influences on their offspring, and when schools seem more receptive to parental involvement. For example, active PI in one program (working with at-risk fifth graders in a rural school) was found to "lead to greater sense of belonging at school, greater confidence in ... academic ability, and increased interest in helping other children," as well as "significant gains in 
self-esteem and self-control" (Smith \& Martin, 1997, p. 19).

\section{Middle School}

As children move into adolescence with its myriad unique challenges of that tumultuous age, the need for PI seems to intensify, and the middle-school-aged child seems to be particularly in need (Barber \& Patin, 1997). Youth, who comprise $20 \%$ of the population, account for $40 \%$ of crimes reported in the U.S. (Walker \& Sylwester, 1991). About half of the youth committing serious crimes are under fifteen years of age ( $75 \%$ of them are boys). Many of those children come from impoverished homes in which adults have limited parenting skills. The environment of such homes often leads to anti-social behavior of boys early in life. A longitudinal study of fifth-grade boys found that more than $50 \%$ of boys exhibiting such anti-social behavior had been arrested for crimes by the seventh grade. Even though the school and other social agencies attempted to address the problems as they were recognized, the behavior was predictive of the patterns revealed as they progressed through school.

A primary cause of the anti-social behavior, not addressed by the school, was determined to be ineffective parenting. Parents were "not involved in their children's lives as playmate, mentor, role model, or advocate. They didn't encourage their children and didn't positively manage or interact with them" (Walker \& Sylwester, 1991, p. 15). Walker and Sylwester concluded, "The single best predictor of adolescent criminal behavior is a long established pattern of early school antisocial behavior" (p. 15). Recommendations drawn from the study include school initiated home/school collaboration in behavioral intervention, parent training programs to help parents develop reward incentives for positive student attitudes and academic success, and the 
development of communication for school/home monitoring of school performance.

However needy the children are of their parents' involvement, there is no doubt that PI decreases as children progress through middle and secondary levels of school (Barber \& Patin, 1997; Cushman, 1993). Many reasons have been offered for the lack of PI at all levels: teacher resistance (Becker \& Epstein, 1982); parental disengagement and lack of opportunity (Steinberg, 1996); parent illiteracy (Rosow, 1991); parent perceived intimidation and lack of respect (Lindle, 1989); unwelcoming schools (D. Cohen, 1995; Cushman, 1993; Steinberg, 1996); lack of time (Feldman, 1994); and misunderstanding by parents and school staffs. In some cases, evidence reveals that planned PI programs were simply not carried out as intended (McLaughin \& Shields, 1987). A sharp decline in PI is noted between elementary and middle school, and at the secondary levels the decline is even greater and the reasons for it more complex, suggesting that parents at those levels feel even less needed or wanted in their children's education (Barber \& Patin, 1997). In work done by Becker and Epstein (1982), it was noted that school staff attitudes toward parents made a significant difference in the degree of corresponding PI. Many teachers in some schools are notorious for posturing against such outsider intrusion.

In one survey (Epstein, 1987), over a third of the parents recorded no conference with their child's teacher during the entire year; $60 \%$ had not talked to a teacher by telephone. Another study (Meade, 1992) reported that only $17 \%$ of parents regularly communicated with their children's teachers.

\section{Religion}

The words "liberal," "moderate," and "conservative" have varied meanings as 
they relate to the political, economic, and social aspects of society. Those monikers are similarly applicable to religion as well.

According to researchers in the field of religion, "liberal" refers to those who "reject firm belief in central tenants of Christian orthodoxy" (Glock \& Stark, 1965, p. 120). Included in the liberal Protestant category are Congregationalists, Methodists, and Episcopalians. Moderates, on the other hand, are considered to be "less secular" than liberals, holding more closely to religious doctrines. They include Disciples of Christ and Presbyterians. For both liberals and moderates, however, there can be considerable variation within the denomination from one local church to the next.

Lastly, conservatives (often called fundamentalists) are recognized as the most religious of all the Protestant Christian groups. These groups include members of Independent Baptists, Seventh Day Adventists, Nazarene (Ellison \& Sherkat, 1997), American Lutherans, the Missouri Synod Lutherans, American Baptists (Glock \& Stark, 1965), Assembly of God, Pentecostals, Holiness, Church of Christ, and Church of God (Roof \& McKinney, 1987). The largest Protestant denomination in America is also considered to be conservative--the Southern Baptists (Sherkat \& Ellison, 1993). In addition, small sects (Glock \& Stark) and cults (Bader \& DeMaris, 1996) are added to the conservative category by some researchers. Sherkat and Ellison (1997) suggest five identifiers of conservative Protestants in their study on opposition to pornography: (a) frequent church attendance, (b) strong stance on Biblical inerrancy, (c) moral absolutism, (d) fear of social contamination, and (specific to their study) (e) opposition to pornography.

Even though the Catholic Church is a Christian organization, its basic doctrines 
and tenants are so different from the Protestant covenants, it is difficult to draw comparisons between the two in terms of liberal, moderate, or conservative labels (Hofstadter, 1963). Because the rural South is known to be disproportionately, predominantly conservative Protestant, and because there are fewer than 1\% Catholic families represented in the DEMS student population (this study), the Catholic question will not be included within the scope of this study. It should also be noted that, for the most part, conservative Catholic families tend to seek out parochial schools for their children to attend and avoid the public schools.

\section{Religiosity}

Religion is generally considered to be one's faith in a supernatural force or being. Religiosity, on the other hand, can be revealed through many facets of religious behavior as well as belief. The more closely a person adheres to the standards of practice and belief, the more religious she or he is determined to be--the higher the degree of religiosity the person exhibits.

At first thought, it would seem simple to know what is meant by "a very religious person." But researchers have apparently grappled with the definition of the term for many years with limited consensus.

\section{Dimensions of Religiosity}

Two of the earlier researchers in the sociology of religion (Stark \& Glock, 1968) presented 5 dimensions of religiosity:

1. Intellectual Dimension--knowledge of the "basic tenants of ... faith and its sacred scriptures" (p. 22).

2. Ideological Dimension--beliefs in the basic tenants of faith.

3. Ritualistic Dimension--practices of faith such as "worship, prayer, 
participation in special sacraments" (p. 22) (e.g., Holy Communion, baptism).

4. Experiential Dimension--“direct knowledge of ultimate reality" (p. 22) or experiencing the divine nature of God.

5. Consequential Dimension--"secular effects of religious belief, practice, experience and knowledge" (p. 23) or how religion impacts a person's relationships with others.

Glock and Stark (1965) also developed an orthodoxy index to quantify the degree of a person's religiosity ranging from complete acceptance of traditional Christian doctrines to complete rejection of Christian doctrines. During the years since the Glock and Stark (1965) study, the dimensions of religiosity have been expanded to include other manifestations of religion other than subdivisions of the five they first identified, but the elements that seem to be consistently identified by researchers are presented here.

Intellectual dimension. Work by Glock (1973a), King and Hunt (1969), Stark and Glock (1968), and Wuthnow (1973) holds that the intellectual dimension is an indicator of religiosity. These researchers assert that knowledge of fundamental doctrine of the faith, of the Ten Commandments, of Old Testament prophets, of selected Bible scripture, and of the Bible's general format is expected of a religious person.

Ideological dimension. Another dimension identified by most researchers as a strong indicator of religiosity is the ideological dimension: the beliefs that individuals affirm. According to many studies (Cornwall, Albrecht, Cunningham, \& Pitcher, 1986; Davis \& Robinson, 1996; Glock, 1973b; Glock \& Stark, 1965; King \& Hunt, 1975; Sherkat \& Ellison, 1997; Stark \& Glock, 1968; Wuthnow, 1973), a strict adherence to the belief that the Bible is the holy word of God, without fault or contradiction (inerrant) and should be taken literally, word for word, is one of the most accurate measures of a high degree of religiosity. 
The dimension of ideology is also manifested in the belief in a supernatural force or being and in miracles. For example, Glock and Stark (1965) hold that an all-powerful Creator is a universal Christian belief. Additionally, they say that the omnipotent Creator is believed to be a personal, omnipresent God. Davis and Robinson (1996) add that a truly religious Christian believes that "the course of our lives is decided by God" (p. 233) and that "God is the ultimate moral arbiter of right and wrong" (p. 242). Sherkat and Ellison (1997) indicate that the persons exhibiting the highest degrees of religiosity could be called moral absolutists, those who believe in standards of right and wrong that are based on God's laws (Davis \& Robinson, 1996).

Other ideological indicators of religiosity are beliefs in the supernatural nature of the Christian faith. These include the virgin birth of Jesus as the divine Son of God; the acts of Jesus walking on water and raising people from the dead; the death and resurrection of Jesus; salvation through the death of Jesus; the existence of life after death; authenticity of other Biblical miracles; and the existence of a supernatural, active devil (Glock \& Stark, 1965).

Some researchers (Sherkat \& Ellison, 1997) contend that very religious believers accept sin as human behavior that is in conflict with Biblical truth, that sin leads to social contamination or loss of social capital, and that sin causes physical and/or mental illness. "True believers" think that physical and/or mental sickness is caused by spiritual sickness (sin)--sometimes the sickness is caused by the sin of our ancestors whose punishment is passed on to subsequent generations (Paloma \& Hoelter, 1998).

Ritual dimension. The next dimension held as an indicator of religiosity is ritual. Glock and Stark (1965) offer "ritual" to mean the practice of religion, the acting on 
knowledge held and beliefs maintained. They identify several actions as evidence of this dimension: attending church, accepting Christ for salvation, being baptized, taking Holy Communion, joining the church, and praying.

Most researchers (Asa, 1995; Bahr \& Martin, 1983; Cornwall et al., 1986; Glock, 1973b; Faulkner \& DeJong, 1966; Harris \& Mills, 1985; Hoffman \& Miller, 1997; King \& Hunt, 1969, 1975; McIntosh, Alston, \& Alston, 1979; Sherkat \& Ellison, 1997; Stark \& Glock, 1968; Wuthnow, 1973) deem church attendance as a good indicator of religiosity. In addition to attendance at church, Glock and Stark (1965), King and Hunt $(1969,1975)$, and Wuthnow (1973) suggest that spending time in other church activities be used as an indicator. Activities might include mission societies, choirs, youth groups, clubs, study groups, fellowship dinners, and others.

Other activities that are considered ritual indicators of religiosity are meditating (Bahr \& Martin, 1983) or having devotions (King \& Hunt, 1975; Stark \& Glock, 1968; Wuthnow, 1973), reading scripture (Asa, 1995; Bahr \& Martin, 1983; Glock \& Stark, 1965), holding church leadership positions (King \& Hunt, 1969), praying publicly (Asa, 1995; Bahr \& Martin, 1983; Glock \& Stark, 1965), praying privately (Glock \& Stark, 1965), contributing to the church (Glock \& Stark, 1965; King \& Hunt, 1969, 1975), and participating in religious activities at home (Cornwall et al., 1986). Many other activities may be cited: e.g., spiritual dancing, weeping, singing, fasting, preaching.

Experiential dimension. The next dimension of religiosity seems to be one not necessarily known by all practicing Christians. In fact, the more intense levels of this dimension are "discouraged or even opposed by the vast majority of religious institutions" (Glock \& Stark, 1965, p. 61). The experiential dimension is "some sense of 
contact, however vague or fleeting, with a supernatural agency ... from the vaguest glimmerings of something sacred to rapturous mystical unions with the divine" (p. 125) (see also Davis \& Robinson, 1996). Glock and Stark (1965) go further to subdivide four types of revelation experience.

1. Confirming Experience--a person senses the reality of a supernatural being that does not apparently sense the person

2. Responsive Experience--a person senses the reality of a supernatural being that acknowledges the person's presence

3. Ecstatic Experience--a person senses a mutual affection between himself/herself and the supernatural being

4. Revelation Experience--a person is a "confidant of and/or a fellow participant in action with the supernatural actor" (p. 127).

The confirming experience occurs when a person makes note of the divine existence. A "generalized sense of sacredness" (p. 44) might take place during rituals like communion, baptism, or marriage ceremony. Or a "specific awareness of the divinity" (p. 44) might be noted with a sense "of Jesus in the room" (p. 45) or a sense of a diabolic presence might be noted with a "generalized sense of evil" (p. 62).

With a responsive experience, "mutual presence is acknowledged" (p. 43) in one of three forms: salvation, miraculous, or sanctioning. During a salvation experience, "the divine has chosen to count [a person] among his own" (p. 46) and a person receives a "sense of being saved in Christ" (p. 47) as his/her sins are cleansed from the soul. A miraculous experience occurs when the divine "actively intervenes in the processes of the physical world" (p. 49) for the benefit of healing, rescue, survival, or economic good fortune. If the divine is sensed to intervene actively to punish or guide in another direction, the experience is considered to be sanctioning. Similarly, the evil presence or 
devil may be experienced through evil temptations, evil miracles, or curses (Glock \& Stark, 1965).

Giving rise to the term "holy rollers," the ecstatic experience describes "an affective relationship akin to love" (p. 43) which produces "a physical and psychological upheaval of intense proportions, similar to orgasm, intoxication, seizures" (p. 53). Such experiences are often expressed by "jumping, shaking, [or] screaming" (p. 53) or by speaking in tongues (Glock \& Stark, 1965).

Glock and Stark (1965) note that a sense of enlightenment accompanies a revelation experience. A person may receive visions--the person sees the divine but the divine "does not acknowledge the presence of the viewer" (p. 55). Or a person may hear voices that allow the hearer to receive messages about the future. If the enlightenment includes information about future events it is categorized as prophetic. Theological enlightenment reveals information about "divine will and character" (p. 58) and commission revelation includes instructions "to take particular actions" (p. 58): for example, to lead crusades, to conduct revivals, or to fight in a holy war.

Consequential experience. The consequential dimension of religious experience is expressed by good deeds, tithing, keeping the Sabbath, loving one's neighbors, etc. (Glock \& Stark, 1965). This dimension goes beyond that of ritual. It is more than simply attending church or participating in church activities. The consequential dimension has to do with putting into action what religion teaches is the correct thing to do as a Christian (Asa, 1995; Cornwall et al., 1986; Glock, 1973a; King \& Hunt, 1975; Perrin, 2000; Stark \& Glock, 1968; Wuthnow, 1973). According to Asa, the consequential dimension may be considered ethical behavior such as, doing good, loving neighbors, 
being honest, or respecting others.

It is commonly assumed that, because of the Christian imperatives to do good and to help others, conservative Christians feel compelled to do what is right and ethical. Researchers Edmond, Shipman, and Cahoon (1993) do claim, "Religious persons are generally actively involved with societal issues related to personal responsibility and good citizenship" (p. 142). This claim has been supported by Evans, Cullen, Dunaway, and Burton (1995), a very recent study by Perrin (2000), and a study on civic membership by Bellah, Madsen, Sullivan, Swidler, and Tipton (1996).

However, all researchers do not agree. Asa (1995) contends that most people who are quite religious tend to be strongly opposed to the "church's involvement with social issues" (p. 30). Asa maintains, "concern for man-to-man ethics is for all practical purposes not a part of general Protestant religious commitment" (p. 181) (see also Roof, 1972). Park and Smith (2000) find that Protestants are either "unaffected or in some cases discouraged from volunteering by their existing religious capital" (p. 283). Likewise, Hirsch and Stark (1969) find that religiosity is not related to incidences of juvenile crime.

Investigation into the apparent contradiction provides some resolution. In essence, Protestant teachings suggest that Christians should be doing good and doing good for others; however, it has been shown that strongly conservative Christians believe social evils will disappear through divine intervention when/if all people become Christians. So, instead of addressing social needs, they concentrate on evangelism and conversion (Asa, 1995), holding that social ills will be remedied permanently through salvation. Watt (2002, p. 28.) further maintains, "Bible-carrying [very conservative] 
Christians are hostile to welfare states and to socialist ones" (p. 28) and believe that "the Social Security system is sinful, that the Federal Reserve System is sinful ... [ and that] income tax is sinful"'(p. 38). Obviously, the consequential dimension of religiosity is difficult to measure because of the multiple perceptions of what "being good" and "doing good" really mean; because of the contradictions and difficulty in measurement, it will not be used as an indicator in this study.

\section{Religion and Secular Education}

Multiple perception of what "doing good" means leads to a dichotomous response to secular education. If, as Glock (1973a) suggests, the consequential experience is realized by Christians who "live" their religion by becoming involved in the world so that "good" might be done, it would seem to follow that those with the highest degree of religiosity would be the most involved in education--so that their children might receive the most good from their involvement. If, on the other hand, as Asa (1995) and others contend, the Protestant religious experience is focused inward on personal salvation, with the belief that evil is defeated when all individuals have been converted, it may well be that these parents will not be much involved in their children's education because that secular concern is secondary to (and dependent on) one's personal relationship to God. The question then becomes, does religious commitment compel parents to become involved in their children's education to advance the ideology of good works, to fulfill moral/ethical obligations, or rather to turn inward (with little direct attention to education) to respond to biblical teachings?

Some evidence of conservative Christian Parent Involvement exists. School prayer is an issue of social morality to conservative Protestants and they have been active 
in promoting its re-initiation into public schools (Hoffman \& Miller, 1997). Conservative Protestant groups have sought to control curricula, texts, and teaching methods so that fundamental Christian values are not threatened (Darnell \& Sherkat, 1997; Kennedy, 1986; B. LaHaye, 1977; T. LaHaye, 1983; Maddoux, 1992). Additionally, orthodox Christians are generally considered conservative on all issues of schooling (Davis \& Robinson, 1996).

On the other hand, evidence even more strongly suggests that conservative, Protestant parents "regard education with enmity" (Sherkat \& Darnell, 1999, p. 30), possibly because of the role education has played in transmitting social values (Darnell \& Sherkat, 1997). Secular curriculum often promotes humanistic values--in direct conflict with conservative Protestant belief that the Bible is the inerrant word of God.

Fundamentalists "subscribe to a vividly otherworldly belief system that is often antagonistic toward secular education" (Sherkat \& Darnell, 1999, p. 24). For them, education serves to "undermine both secular and divine authority by promoting humanism and denigrating faith" (Darnell \& Sherkat, 1997, p. 307). Modern education practices strongly support the inquiry method, which teaches students to question everything. Critical thinking skills teach children to challenge mentally everything they are taught, in school and out. The scientific method, included in most tests of educational skills, rejects acceptance of any reality based solely on faith (Ellison \& Musick, 1995). There is little wonder that, when the beliefs and values taught by educational institutions so strongly conflict with conservative religious values and literal interpretations of Bible scripture, opposition to public education is especially vehement (Darnell \& Sherkat, 1997). A prime example is the study of evolution as the central organizing principle of 
biology instruction. Evolution, or Darwinism, is a clear affront to fundamentalists' belief in the Genesis creation. Their consequent strategy of designating creationism as "Creation Science" is an effort to have science classes taught with equal time given to both "theories," Creationism and Darwinism.

Though "more tolerant views among conservative Protestants have been tied to increasing education levels" by some (Hoffman \& Miller, 1997, p. 57) (see Exchange Theory, below), Bader and DeMaris (1996) find that each year of educational attainment increases chances of cult membership. This finding contradicts traditional thought unless it is considered in the light of typical adolescent rebellion and acceptance of their own knowledge (education) as superior to that of their parents. On the other hand, apparently diametric to Bader and Demaris, Asa (1995) reports that more education increases the likelihood of a subject going from "religious belief to no religious belief" (p. 79). But, again, if viewed in the light of typical adolescent rejection of parental ideals, the denial of a parent's religion after being subjected to modern educational theories is understandable and predictable. Iannaccone (1990) finds support for the contention that higher levels of education negatively affect traditional religious beliefs, and Sherkat (1997) also finds that the higher the educational attainment the more likely affiliation with religious organizations will be severed. It is apparent that conservative religious groups have reason to feel threatened by and suspicious of education goals and methods.

Indeed, conservative Protestant authors demonstrate "antipathy to secular education" (Sherkat \& Darnell, 1999, p. 24), claiming that "the whole education system has been taken over by an atheistic, humanist philosophy that is largely anti-God, antimoral, and anti-American" (p. 25). According to a report in a Baptist newsletter 
("Schools," 1999), Christians are pulling their children out of public school because of school violence, poor discipline, and inferior academics, and they have increasingly offered alternatives to public education. For example, Rescue 2010, based in California, seeks to promote Christian education. Exodus 2000, led by E. Ray More, has been endorsed by Tim LaHaye, a conservative Baptist pastor. The Exodus Project of Minnesota issued a statement urging members of the American Family Institute to withdraw their children from public schools. The Separation of School and State Alliance, founded in 1994, strives to eliminate government involvement in schools. Because secular education is considered "antagonistic to fundamentalist Christians' religious values" (Darnell \& Sherkat, 1997, p. 309), "fundamentalist parents and denominations may not encourage children to excel in secular studies, may discourage educational aspirations, and may direct children away from college" (p. 309). In fact, Darnell and Sherkat find that "fundamentalist orientations significantly retard educational attainment above and beyond the level predicted by social background factors alone" (p. 313).

\section{Outcomes Based Education}

Particularly repugnant to the conservative Christian is Outcomes Based Education (OBE), which was introduced to Kentuckians by the 1990 Kentucky Education Reform Act (KERA). The American Family Association, a national fundamentally conservative religious "news magazine" based in Washington, DC, reprinted the Phyllis Schlafly Report of May 1993, advocating the elimination of OBE. Of the ten major objections to OBE given in the report, the first was the perceived aim of OBE to mislead parents purposefully. Other objections to OBE that the report presented included the use of 
students as "guinea pigs" in experiments, the "dumbing-down" of curricula, the teaching of tolerance for alternative lifestyles, and the computer tracking of students.

Rural conservatives widely claim that many educational reforms have a valuebased, anti-god-secularism, anti-family-values, anti-country, anti-Bible agenda. Much of that criticism is directed at outcomes based education, in spite of the fact that this is simply a process for measuring what a student knows apart from the seat time accumulated (Spady, 1981). Proponents of OBE maintain that it came from the behavioral science tradition ("OBE," 1995): "State what you want students to be able to do in measurable terms, then design curriculum that lets them learn how to do it" (p.11). Yet, the conservative critique continues mostly unabated. Values clarification exercises, which encourage the participants to wrestle with the relative priority of values, are often cited as teaching the values of secular humanism (Hunter, 1991). Oddly enough, $80 \%$ of the anti-OBE public surveyed indicated they wanted clear goals with curriculum designed to help students to meet those goals, still many of them claimed that $\mathrm{OBE}$ was "Organized Satanism."

With the arrival of the Kentucky Education Reform Act (KERA) and subsequent mandated reforms, rural conservative Kentuckians were alerted to the invasion of godless-outcome-based education movements that threatened the rural values they so reverently upheld. Local radio talk shows aired pronouncements (from James Dobson, "Focus on the Family") deriding OBE as a satanic tool of moral destruction. Community leaders held much-attended forums and town council meetings to prepare for and defend against the evil invasion. In one Western Kentucky school district near the author's home, a superintendent instructed science teachers to glue together the pages of the 
textbook that explained evolution.

Primarily conservative Christians, national anti-OBE leaders organized rallies to attack OBE (Needham, 1995), calling it an instrument of Satan. It is interesting to note that, despite public outcries, extensive and intensive parent involvement in children's education is one of the stated goals of KERA (Boysen, 1992) and parents from all over the state of Kentucky were used in every process of planning and structuring of all KERA documents, procedures, and testing protocols.

Petitions calling for repeal of KERA/OBE were widely and vigorously circulated. One petition (see Appendix C) states: "We the People of the Commonwealth of Kentucky are concerned citizens and parents. We are grieved by the type of education reform mandated ... [and] by the attitude changes in our children, by the significant changes in the classroom, by the experimental nature of the reform, and by the deemphasis given to basic academic education in favor of social re-structuring" ("A Petition," 1994). The petition goes on to claim that KERA/OBE is based on the philosophy of "The New World Order, Social Restructuring, Multiculturalism, World Citizenship, the New Age Movement" and other belief systems that threaten "Religion and Family Sovereignty."

Another petition (see Appendix D) warned: "KERA is intended to shape all children's values to conform to those planned by the state" ("Educating," 1994). It also proclaimed that most teachers are against KERA because KERA is an attempt to turn Kentucky into Communist China through techniques designed to "equate your children and grandchildren with rats in a maze. You've heard of Pavlov's slobbering dogs ... [KERA is] casting a dark cloud over the future of your children and grandchildren." 
Mark Schaver (March 23, 1994), staff writer for the Louisville Courier-Journal, reported the delivery of a petition to the Kentucky General Assembly by Steve Miller (not the dissertation chair), representing America Awake, a group based in Paducah. The petition boasted 11,000 signatures calling for the repeal of KERA because it teaches "values at the expense of academics" and promotes "witchcraft and homosexuality."

Kentucky is not alone in its battlefield status. Conservative elements in one Michigan school mobilized to stop education reform movements (Detwiler, 1994) because they were identified with "New Age religion, secular humanism, moral relativism, one-world government, invasion of family privacy, witchcraft, satanism, and anti-Americanism" (p. 24). Speakers invited to public meetings sponsored by the opposition were "two local conservative Evangelical Christian ministers, the staff director from the Christian Family Centre and K-12 school, a local attorney[,] . . a gospel singer who presented herself as an educator, and a political staffer from the office of the local state representative--a former Fundamentalist minister" (p. 26).

In South Carolina, Christian conservatives were called to battle when education reformists threatened to "sabotage home-taught moral and religious values" (Smolowe, 1993, p. 34). Advocates of education reform accused the "Christian foot soldiers" of a "moral crusade against gay rights, abortion, cultural diversity," and any other policy they deemed as wrong. Those "Christian foot soldiers" even criticized steps the school system took toward hiring a school nurse because they were seen as in the direction support of abortion, and they considered Pumsy, the school counselor's Dragon puppet, to be demonic.

Elementary school counselors across the United States were also attacked (Pate \& 
Rooney, 1994). Groups who identified themselves as conservative Christians angrily protested counseling programs that attempted to help students and parents understand and tolerate diverse beliefs. The protestors viewed values discussions as undermining parental authority, and they did not want values to be taught as situational or interpretational, especially concerning issues of homosexuality and abortion.

The source of many of these calls to battle is not difficult to identify. The nation's largest and most powerfully organized religious entity, the Southern Baptist Convention, has openly supported them, financially and otherwise. Leaders of the Southern Baptist Convention are usually outspoken and politically active, e.g., Jerry Falwell, Tim LaHaye, James Dobson, Phyllis Schlafly.

However, while it would seem at first obvious that a desire to do good--a consequential religious value--would cause fundamentalists to become involved in their children's education, other evidence suggests that the cost of involvement is just too great for religious conservatives. Loss of faith, contamination of spirit, undermining of authority, denigration of biblical truths, and threat by social institutions are seen as challenges to their way of life that are largely promoted by education. Moreover, Sherkat and Ellison (1999) suggest that "marginalized groups" may find "free-space" within their church that allows them social networks supportive of common interests, needs, identities, articulation opportunities, and agendas.

\section{Prosperity}

One area deemed indicative of religious conservatism by some is not so easily categorized as such. The word "conservative," as it applies to religion, usually means holding close to biblical mandates, standards, and literalism: religious conservatism. 
"Conservatism," as it applies to society, may mean having or acting according to "higher" or more rigid moral Judeo-Christian or traditional standards of behavior: social conservatism. But, the terms "economic conservatism" and "political conservatism" (political conservatism will be discussed more fully in the next section) take on financial connotations that often appear confusingly dichotomous to other meanings of "conservative." A financial conservative or political conservative is generally believed to be of the upper class with more money to spend and to be taxed.

Be it irony or hypocrisy, though religions such as Presbyterians are considered religiously liberal (Coreno, 1998), they have also revealed financially conservative tendencies. They spend fewer dollars on social issues and missions. At the same time, their congregations tend to belong more often to middle, upper middle, and high income categories. In contrast, conservative Christians have traditionally believed that prosperity "would defile them" (Sherkat \& Darnell, 1999, p. 32), leading them to pursue things of this world rather than things of the next. The Puritans are well known to have embraced poverty and simplicity in their manner and way of life. In the modern era it is not difficult for most rural people to remember the old country preacher who was required to work a regular job during the week and preach only on Sunday or every other Sunday. Yet, some Protestants of the most conservative denominations come from the lower economic levels, but they are the most giving, or most liberal with their money, of all the Christian denominations (Coreno, 1998).

It follows that because higher levels of education do lead to prosperity for the individuals willing and able to pursue those higher levels, secular education is more than a little suspect to the religiously conservative congregation. Recognition of this posture 
leads to understanding of the substantial weight of the anti-education, anti-intellectualism literature produced, especially in southern, rural cultures, over the decades since government became involved in education. Indeed, anti-intellectualism appears to be a strong force alive and well in the rural South today, as is discussed in the following section.

\section{Devaluation of Intellectualism}

"The Puritan clergy founded the tradition of New England intellectualism; and this tradition, exported wherever New Englanders settled in large numbers, was responsible for a remarkably large portion of the country's dynamic intellectual life throughout the nineteenth century and into the twentieth" (Hofstadter, 1963, p. 399). But, the point needs to be made: the goal of the Puritan educator was to train Puritan children to read the Bible and to remain knowledgeable enough to maintain the religious freedom they had gained at extreme expense. While recognizing education as a parochial innovation in America, beginning with the Puritans in the Massachusetts Bay Colony, the classic study by Richard Hofstadter, Anti-Intellectualism in American Life, connects American religious fundamentalism through the 1800 s to Anti-Intellectualism. Hofstadter credits the Great Awakening, which began in 1720, with the emotional revivalism that preached a message of salvation through the spirit, not through learning. The Awakening, he claims, brought an end to the Puritan age in America and began the age of evangelism. He also believes that "the effect of the Awakening was to subordinate education to religious factionalism" (p. 72). For, "the Puritan ideal of the minister as an intellectual and educational leader was steadily weakened in the face of the evangelical ideal of the minister as a popular crusader" (p. 86). Supported by numerous examples of 
the monumental impact that Anti-Intellectualism (AI) has had on conservative Christians, Hofstadter contends that intellect came to be viewed as the antithesis of God.

In addition, with the rise of democracy and the adoption of our Constitution, Hofstadter (1963) asserts, "Widespread belief in the superiority of inborn, intuitive, folkish wisdom over the cultivated, sophisticated, and self-interested knowledge of the literati and the well-to-do" (p. 154) grew rapidly. Ultimately, the "disestablishment of a patrician leadership" was completed during Jacksonian Democracy when it became the goal of the common American "to build a society that would show how much could be done without literature and learning" (p. 51). Because intellect was "resented as a kind of excellence, as a claim to distinction, as a challenge to egalitarianism" (p. 51), resentment was most noted in lower class, fundamentalist religions which "preached a religion of the poor ... against learning and doctrine" (p. 56).

By the late 1800s Darwinism had reached the high schools, and the fundamentalists struggled to "save the religion of their children ... from the ravages of the evolutionists, the intellectuals, the cosmopolitans" (Hofstadter, 1963, p. 126). "On one side were the voices of the people and the truths of the heart; on the other were the intellectuals, a small arrogant elite given over to false science and mechanical rationalism" (p. 127).

About the same time, popular thought began to connect intellectualism with homosexuality. According to Hofstadter (1963, p. 186), many began to argue that "culture is impractical and men of culture are ineffectual, that culture is feminine and cultivated men tend to be effeminate." Attacks were made upon those educated by "English-oriented prep-school personnel of the State Department, associated with charges 
of homosexuality" (p. 189).

Additionally, intellectualism was connected to Communism. Hoftstadter (1963, p. 226) recalls newspaper reports that spoke in favor of the masculine, military leader Eisenhower over the intellectual, educated, effeminate Stevenson in the 1952 presidential campaign, because it was well-known that university people had been fed "strong leftist Socialistic ideas, as well as with Communistic loyalties." At that time, the fundamentalist mind believed that "socialism is nothing more than a variant of Communism, which, as everyone knows, is atheism" (p. 135).

Formal education was also regarded as a threat to becoming a "self-made man," an idea that was "a historical outgrowth of Puritan preachings and of the Protestant doctrine of the calling" (Hofstadter, 1963, p. 254). Especially among Baptist groups, hard work, sincerity, evangelistic spirit, and good common sense took precedence over education, intelligence, and even Bible knowledge in assessing a preacher. Hofstadter quotes one of the most remembered Baptist preachers, Billy Sunday, to illustrate: "The church in America would die of dry rot and sink forty-nine fathoms in hell if all members were multimillionaires and college graduates" (p. 116).

Because of pre-World War I isolationism, Hofstadter (1963, p. 48) says, "the heartland of America filled with people who are often fundamentalist in religion, nativist in prejudice, isolationist in foreign policy, and conservative in economics." He adds, "Protestant individualist culture still so widely observable before the First World War ... had to confront modernism in religion, literature, and art, relativity in morals, racial equality as a principle of ethics and public law, ... the endless sexual titillation of our mass communications[,] . . Darwinism[,] . . Freudianism, [and] Marxism" (p. 43). 
Public response to these disclaimers toward public education resulted in a push toward vocational training so that youth could obtain "some useful occupation" instead of becoming “too many 'gentlemen' and 'ladies"” (Hoftstadter, 1963, p. 258). Even traditional religion moved toward "a curious cult of religious practicality" (p. 265). Moreover, the promoters of this practical culture expanded their ideas to include other facets of life. For example, intellectual pursuits could lead to idolatry of reason and a scorn of religion. Intellectual interests could lead to divorce because "a liking for philosophy, psychology, radical politics, and for reading the New Republic are much less auspicious for marital bliss than a liking for Y.M.C.A. work ... [and] Bible study" (p. 270). Also, of course, most teachers have heard the old adage: "Those that can, do; those that can't, teach; those that can't teach, teach teachers."

As a result of this long-held Anti-Intellectual stance, members of conservative religions tend to place less value on educational attainment (Darnell \& Sherkat, 1997). The "anti-intellectual atmosphere of the household may . . . hinder attainment" (Sherkat \& Darnell, 1999, p. 26). Darnell and Sherkat (pp. 310-311) find that "conservative Protestants and Biblical inerrantists have significantly lower educational aspirations than other respondents ... [and] significantly lower educational attainment." They go on to add, "Fundamentalist orientations significantly retard educational attainment above and beyond the level predicted by social background factors alone" (p. 313).

Anti-education sentiments, furthermore, have been found to be even more profoundly evident (Sherkat \& Darnell, 1999) in lack of parental support as well as in instances of punishment. Deviant male children are often not allowed to take classes such as auto shop or carpentry or other preferred class as a form of punishment for their 
deviation from the parents' religious conservatism. Such punishment may create a "hostile environment for maintaining educational aspirations" (p. 24) and "may create anti-intellectual preferences in children who do not follow their parents' faith" (p. 24). It has been established, also, that "education of nonfundamentalist women is significantly hampered by fundamentalist parents." On the other hand, Bible-believing, compliant female children and "non-rebellious male children" (p. 24) seem to suffer no significant interference to parental support of their education. Furthermore, fundamentalist parents significantly "boost the educational attainment of male children who believe that the Bible is the inerrant word of God"' (p. 24).

In other words, male and female (to an even greater degree) children who dare to challenge the home faith are put at educational risk by parent withdrawal of educational support. Conversely, compliant female children and nonrebellious male children seem to experience no interference to parental support. Moreover, male children who readily embrace the home faith are strongly encouraged in their educational pursuits.

Interestingly, Bjarnason (1998, p. 752) finds that "adolescents who perceive their parents as supportive and caring, report higher levels of religious participation, religious orthodoxy and divine support, as well as perceiving the social world more as a [sic] exterior, constraining reality."

In summary, it may be said that, in some aspects, Christianity teaches believers to become involved in worldly issues and to do good. But when the threats of the secular world are too great, and when the "good" seems to be more of this world than of the next, fundamentalists do resist becoming involved in social activities, including the secular education of their children. 
"The United States was founded on an insistent rusticity. Thomas Jefferson envisioned the country's stability and prosperity growing out of the labor of the yeoman farmers ... and ... hoped that nearly all Americans would stay up on the farm" (Ching \& Creed, 1997, p. 13). But rusticity has lost much of its attraction to the intellectual element of American society including those living in the rural South. Indeed, "rural," in many cases, now imbues pejorative connotations. But what exactly is "rusticity," and who is "rural"?

"Rural" is sometimes termed "non-metropolitan." It usually refers to an area of fewer than 2,500 people living in a community or fewer than 1,000 people living within a square mile. It may also include the culture of the people living outside the urban and suburban communities. People who live on farms or work in farm-related industries are often called "rurals." Of $115,681,202$ employed people in the 1990 census, only $2.5 \%$ were employed in farming, forestry, and fishing (DeYoung \& Laurence, 1995; Roberts, 1995). Though $24.8 \%$ of Americans were living in rural areas, less than $2 \%$ were living on farms. That means, "fewer than one in fifty Americans lives on what the census broadly defines as a farm" (Roberts, p. 9).

Who are these Americans? What are they like? What do they value? Rhoda Halperin, a Kentucky anthropologist differentiates "shallow rural" from "deep rural" but most scholars do not recognize such a distinction (Creed \& Ching, 1997), and they often generate a picture of a quaint little rustic farmhouse in disrepair, surrounded by broken wagon wheels and docile farm animals to embody "rural." But rural means much more than rusticity (Ching \& Creed, 1997). 


\section{Southern Rural Conservatism}

Rural Values in the South mirror many of the values held by conservative religions in the South. For instance, Rural Values reflect strong Social Conservatism and Traditionalism (SC/T). Rural Southerners embrace a "pious poverty" and an accompanying Anti-Intellectual (AI) posture, also seen as a conservative religious value. Paralleling the religiously conservative tendency to withdraw from the world rather than be a part of it, Southern "Rurals" tend to withdraw from the rest of the world, becoming Ethnocentric or Community-Centric (E) (Little, 1998; Little \& Miller, 2001). Seeing themselves as "part of" their rural "place" or regional culture, rural Southerners have also assumed the God-endowed role of Stewards of the Earth (SE). Finally, a natural association with the $\mathrm{SC} / \mathrm{T}$, religious conservatism, and $\mathrm{SE}$ encourages rural Southerners to manifest or adopt the value of the "Good Old Boy" (GOB) concept.

In the rural South can be found strong support for the Moral Majority, the Christian Coalition, and the Christian Right (Davis \& Robinson, 1996). Rural Southerners also tend to be disproportionately Conservative Protestants (Sherkat \& Ellison, 1997) who are "more likely to believe that the Bible is the inerrant word of God" (Darnell \& Sherkat, 1997, p. 312) and who "want schools to be godly places that practice prayer, teach creationism, and foster other religious beliefs" (Seal \& Harmon, 1995, p. 122).

In their research of insider documents of Conservative Protestant parenting literature, Ellison and Sherkat (1993) found that they held strict-authoritarian attitudes that reflected belief in biblical inerrancy and a literal belief of punishment for the original sins of Adam and Eve. A following study of obedience and autonomy (Sherkat \& 
Ellison, 1993) specified two of the variables to be native southerners and rural natives. As a result of their study, they call for further investigation of conservative Christians to determine how authoritarian parenting attitudes affect academic outcomes for their children.

In a recent study by Little (1998), Rural Values clearly influence decisions that rural school districts make in personnel selection. The four elements chosen by Little to represent rural selection values are "Ethnocentrism [later designated CommunityCentrism in Little \& Miller, 2001], Traditionalism, Primary Group Preference, and Social Conservatism" (Little, p. 64). Community-Centrism, refers to "community superiority," our community is better than your community. Traditionalism indicates "resistance to change" and the exaltation of an earlier time or era. Ethnocentricity $(\mathrm{E})$ is used to designate the attitude of "our kind of people is better than your kind." Primary Group Preference is closely akin to Asa's (1995) communal involvement that is discussed earlier in this study, "the meeting of associational needs by primary type contacts with family and neighbors, as opposed to the greater freedom and diversity of an urban pattern of association" (p. 64). Finally, Social Conservatism refers to the trend for "avoiding that which is different from oneself and preferring a strong, central authority in control of situations" (p. 64).

In another study on conservative Protestant opposition to pornography, Sherkat and Ellison (1997) specifically flagged respondents who were born in and still lived in the South or in rural area. Their study revealed, "Native Southerners have somewhat stronger anti-pornography sentiments than do respondents from other regions of the country" (p. 969). They explained their stronger opposition by the "disproportionate 
concentration" (p. 972) in the South of conservative Protestant denominations. Similarly, a study by Darnell and Sherkat (1997) found that "parents from the South and rural areas are more likely to believe that the Bible is the inerrant word of God ... [and that] youths from the South and females are more apt to hold fundamentalist beliefs, net of other factors" (p. 312).

\section{Poverty}

"Rural" often means undereducated and impoverished. Assessed most often as a monolith, rural areas tend to have a less educated, more educationally at-risk population. They have a higher percentage of "poor school districts, and fewer dropouts who return to finish high school" (Smith \& Martin, 1997, p. 15). People who live on farms register the lowest levels of formal schooling even though there is more demand for agriculture employment that requires technological know-how. Many farmers "cannot operate or repair most machinery or keep pace with the most rudimentary advances in genetic engineering without being able to read" (Roberts, 1995, pp. 235-36).

"Rural" also often means poverty. Rural areas tend to be the most "chronically poor counties in the nation" (DeYoung \& Laurence, 1995, p. 106). The 1990 census reveals the mean farm self-employment to be $\$ 10,064$ (Roberts, 1995), about threefourths that of urban families (Herzog \& Pittman, 1995). Of the ten poorest counties in America, all are rural and "more than half their residents are poor." Kentucky has one of those ten counties (Roberts, p. 222). Kentucky also ranks fifth in the U.S. in percentage of impoverished single-mother families, sixth in lowest percentage of median household incomes, and fourth in lowest percentage of adults with high school degrees (Roberts.)

\section{Anti-Intellectualism}


changing over time. While the gender issue clearly needs more work, there is some support that the rural male is more pejoratively perceived than the rural female. Further support of this is the fact that it is "OK" for rural girls to go to college to become teachers or nurses especially if they return to the local community to work. This AntiIntellectualism phenomenon parallels in many ways that same kind of resistance that is prevalent in the southern, conservative, rural culture. It might be said, after considering the myriad of Anti-Intellectual facets of the southern rural culture, "to achieve is to be white, female, affluent, and urban."

Though ethnicity is usually considered an indication of one's country of origin, it seems to be, in the case of the rural southerner, a designation of one's region of origin. Few typical know their country of origin and do not seem to care. They are southerners-"and damn well proud of it!" The hillbilly caricature is easily identifiable by almost anyone who views it, and the rural southerner accepts the disparaging appellation as part of his/her heritage. It is in the light of the common, perhaps global, designation of a rural American southerner as a "redneck" or "hillbilly," that the distinction of Ethnocentric seems appropriate.

\section{Stewardship of Earth}

According to many students of rusticity, the importance of "place" to a rural Southerner is paramount. Rural Americans (Kentuckians) view themselves as independent, autonomous, resilient, steadfast, and endangered (Porter, 1973). Connection to place is an undying element of being rural ("You can take the man out of the country, but you can't take the country out of the man"). According to Anne Barrett Batson (2002), "We Southerners do have a proprietary sense of belonging and allegiance 
to our Southland and people. (We're special!) Steeped in the rural tradition, strengthened by a heritage of setback and hard-ups, and bound by a fierce love for God, family, and the land, this place is located in the collective heart and soul of all Southerners--wherever we may be" (p. 14).

Inherent in that sense of place is the strong Rural Value of stewardship-Stewardship of the Earth (SE)--because rural people feel obliged to take care of the earth (Howley \& Howley, 1995). As far into history as Saint Francis of Assisi (1182-1226), care of the land and the animals upon it has been taught and affirmed (Witte, 1995). Rural people leave little doubt about this obligation as they call upon Bible scripture to support it:

God blessed them and said to them, "Be fruitful and increase in number; fill the earth and subdue it. Rule over the fish of the sea and the birds of the air and over every living creature that moves on the ground." Then God said, "I give you every seed-bearing plant on the face of the whole earth and every tree that has fruit with seed in it. They will be yours for food. And to all the beasts of the earth and all the birds of the air and all the creatures that move on the ground-everything that has the breath of life in it--I give every green plant for food." (Genesis 1:28-30)

A scholar of 1430 said, "The Bible was given to mankind when it lost the capacity to read the book of nature" (Verhoog, 1995, p. 45). In 1500, Mother Earth was spoken of as a goddess, "the guardian of creation, the daughter of God" (p. 45). Those who tended nature were considered guardians of Mother Earth. Even yet, in Western philosophy, exists a core belief that the tenders of nature are accountable to God "in whose name we manage the earth" (Zweers, 1995, p. 79). Following the Pilgrim's first successful harvest in the New World, they held a celebration to thank God for his blessings on them, the agricultural stewards of the land; today that celebration continues as Thanksgiving Day (Verkleij, 1995). 
Verkleij (1995) explains that as imago dei, the creation of God's image, man is the representative of God in caring for the rest of creation, "in the daily practice of agriculture" (p. 62). Subduing the earth, "dominium terrae is deeply anchored in Christian tradition...." The commission given to man is to be a steward of nature, "to minister to it, in order that future generations can enjoy its fruits" (p. 62). Rural people tend to assume that role willingly. In addition to the acceptance of earth stewardship as a God-given directive, rural people value the "economic importance and cultural distinctiveness of the countryside, where food is produced and human life sustained" (Ching \& Creed, 1997, p. 1). Specifically, the earth-tenders frequently consider themselves as worldly patriarchs.

\section{Good Old Boy}

The lofty title "worldly patriarchs" introduces a pervasive acceptance in the rural South of the religiously conservative position on male superiority (Watt, 2002). Through centuries of religious rural/conservatism, the position has evolved into a southern rural system often affectionately referred to as the Good Old Boy System (GOB). Paul Hemphill (1974) speaks of "tent revivalists," fat-bellied sheriffs," "governors with the audacity to tell the multitude they have 'three friends in this world: God, Sears \& Roebuck, and Eugene Talmadge,"” and "Junior Johnsons." He says that "if you don't know what a good old boy is, you never will--and he could be a mean son of a bitch: half-educated, vengeful, regressive, sadistic and, by all means, a racist.” Hemphill continues: "He could kill a dozen people with bad moonshine, then come out against fluoridation as a Commie plot to poison the water. He could teach his kids hymns on Sunday morning ('Red and yellow, black and white, they are precious in His sight ...') 
and string up the janitor that evening after prayer meeting" (p. 13). In contrast, Hemphill quotes Tom Wolfe: "A good old boy ... is a generic term in the rural South referring to a man ... who fits in with the status system of the region. It usually means he has a good sense of humor and enjoys ironic jokes, is tolerant and easygoing enough to get along in long conversations at places like on the corner and has a reasonable amount of physical courage..." (p. 15).

Donnie Johnson, a reporter for The Free Lance-Star in Fredericksburg, Virginia (2001), offers his version of what a GOB is. "On the opening day of deer-hunting season, as I stood beside an old oak tree, my trusty $.30-30$ rifles by my side, I tried to come up with an accurate definition .... The person ... is always a man, usually of good breeding, whose family either has or has had money. In almost every case, he is a person of considerable influence, usually a politician. And he is generally perceived to be a bit overweight in both stomach and ego." A more pejorative image of GOB is noted in a recent (Concerned, 2002) appeal for help from a man who identifies himself as Concerned Father. In a posting made to a Louisiana attorney web site, the man says, "What does one do when the $\mathrm{x}$-father-in-law, and $\mathrm{x}$-spouse have political, police, and courthouse contacts and uses them? Louisiana "Good Old Boy-System at it's [sic] best! ... I have the facts. Lawyers and the court system is [sic] getting rich off of me... It seems that with the $\mathrm{x}$-father's inside contacts and getting them to delay will break me so they can then get whatever they want. .." (Louisiana, 2002).

According to Judy Johnson (1992, p. 1), the GOB system is "alive and well in our society." It is recognizable in "sexual harassment, lack of women in managerial positions, political themes, lower pay, philandering husbands, Hill vs. Thomas, The 
Thelma and Louise Finishing School, as well as many other examples."

The GOB is a set of mostly unspoken rules of behavior based on a few widely understood and accepted concepts: patriarchy, use of rural talk, rebellion against anything urban or government endorsed, and gun ownership.

\section{Patriarchy}

The rural South is a patriarchy. As part of the GOB creed, it is accepted that boys are valued more than girls; men are assumed to be superior to women (Johnson, 1992). According to Ching and Creed (1997), women and minorities experience a different kind of inequality in the country and values include being male and having male children. Support for this position is often offered through biblical scriptures and traditional attitudes.

Rural Talk

In response to prejudice, rural dwellers have adopted a well-documented posture of rebellion that is expressed in several ways (Ching \& Creed, 1997; Fox, 1997). Ching and Creed (p. 11) maintain, "Self-consciously rustic people often become emphatically anti-intellectual." Reaction against the urban-endorsed and proffered loftier forms of culture (e.g., museums, theatre, opera, knowledge of foreign language, classical music and art) is vented most often through the male use of "rural talk."

The male's ability to know how to talk "country" and to appreciate country music (Ching \& Creed, 1997) is definitely a Rural Value. Maxine Grossman (2002) asserts that for country music, "the only acceptable messages are those that can be harmonized with the implicit Protestant Christian sensibility" (p. 83). In her critique of contemporary country music, "Jesus, Mama, and the Constraints on Salvific Love in Contemporary 
Country Music," Grossman compares "the verse-chorus song" pattern of most country music, "in which a first verse sets the scene, a second verse expands or extends the narrative, and a third verse offers a reinterpretation of it of the narrative" (p. 93), to the salvation experience in born-again, Christian testimony.

Grossman (2002) claims that country music, which began in the rural South and was soon associated with the rural working class, almost always embraces the same religious conventions: "connection, sacrifice, and, ultimately, transformation" (p. 109). At the same time, she postulates, country music champions rural values: the working man, down-home values, blue jeans, cowboy boots, tender-and-tough masculinity, chauvinism, substance over style, love, dedication, sacrifice, self-reliance, compassion, "hypertrophied redneck pride," patriotism, "appropriate sexual standards, family relationship, and gender norms that tends toward the socially conservative ... a general distrust of government authority; and first, last, and always, an emphasis on sincerity, honesty, and authenticity" (p. 87).

According to Grossman (2002), country music's historical connection to gospel music is first "grounded in the salvation of the sinner through the love of Jesus Christ" ( $p$. 88) just as a man's salvation may be realized through the love of a good woman. Second, the Christian dogma that the cure to problems of humanity can be found in personal transformations is mirrored in country song through revelations of "seeing the light," being "baptized by the Spirit," coming to understand that doing wrong is not good, or gaining a victory through painful struggle. Third, Grossman contends, country songs are based on an assumption that "salvific transformation occurs only when a connection is made" (p. 89) and when the connection involves an element of sacrifice. Sacrificial 
elements in religious music are the Lamb, the cross, and Jesus. In country music, sacrificial symbols include long-suffering wives and parents who endure pain to give to their children unselfishly. Particularly sanctified, perhaps even deified, in country music is "Mama."

Grossman (2002) also points out that most country lyrics are written from the male perspective. Via "man talk" they tell the story of a man, placing him first in childhood, finding him again in a wayward adulthood, then, in the final verse, following him through a life-changing experience. In the inner city the use of "macho language" is called "playing the dozens." In rural areas it's called "shit talk" (Fox, 1997). In spite of their self-proclaimed conservative Christian values, Fox (1997, pp. 112-113) finds that rural males relish the opportunity to talk "shit," especially between males gathered in typically male congregation spots, like the local bar. Fox relates his encounter in the field with men engaged in the act of "shit talk": "Talk is an art form in and of itself in the 'redneck' social world. Talk is ironically figured as trash: 'Aaaaaaah, we jus' Assholes talkin' shit,' old Rusty once told me, dismissing my attempts to analyze the intricacies of his verbal creativity. ..."

The rebellious posture is popularized in the rural talk of country song (Fox, 1997, p. 116): "Working-class 'rednecks' continue to clutch at 'country' song even in its most apparently debased and diluted form .... Song is something worth fighting for as a form of participatory experience and practice." As tools of rebellious expression, those country songs embrace the self-conscious Anti-Intellectualism as illustrated in many country songs, according to Ching and Creed (1997):

Charlie Daniels (1990) follows a lyrical call for "a few more rednecks"' with the threat that "you intellectuals might not like it but there's nothing you can do" (p. 
11).

Aaron Tippan (1993) glorifies the "pride, honor, and dignity" of the "working man's Ph.D" (p. 11).

Waylon Jennings describes his rural characteristics as "too dumb for New York City, too ugly for LA" (p. 14).

Southern writers, such as James Dickey, Bill Emerson, Marshall Frady, Paul Hemphill, and Willie Morris (Hemphill, 1974) have been accused of "being charter members of a literary set known as the Pork Chop Conspiracy ... 'listening to hillbilly music with wild, lustful eyes and slobbery, foamy lips"” (p. 10). Included in Appendix G is an example of the electronic "information" notices concerning Southern talk ("Windows," 2002) that circulated among residents of the rural community under study. The obvious self-denigration that is used as humor, Jeff Foxworthy-style (1995), in this document provides explanation for the "Southern Edition of Windows" that "may have accidentally been shipped outside the South." The Recycle Bin is labeled "Outhouse" and Stop is labeled "Kwitit." Along the same line is the electronic mail that affirms "Things a True Southerner Knows" (2001) (Appendix H): "The difference between a hissie fit and a conniption fit," "You should never loan your tools, pick-up, or gun to nobody," and "A good dog is worth its weight in gold."

\section{Rebellion}

Anti-Intellectualism is a form of rural rebellion against urban assessments of rural as uncultured, ignorant, or redneck. Related research has shown rural schools to have lower levels of educational attainment, less equipment, inadequate facilities, shortages of qualified teachers, weak economic bases, and fewer services available (Carlson, 1992). Furthermore, Borland and Howsen (1999) demonstrated that lower population density is 
also associated with lower achievement, a finding that buttresses Carlson's work.

Yet, rural rebellion goes beyond these demographic and resource issues, manifesting in cultural forms such as artistic expression and self-denigration. Illustrated in the section (above) on Rural Talk, artistic expression can be realized as a form of rebellion by considering the sentiments shared in songs and literature (also noted above). Almost always proclaimed by a male artist, being redneck is considered a rank of status, part of the GOB code (see James Dickey's novel, 1970, Deliverance, and Erskin Caldwell's, 1995, God's Little Acre).

In addition to patriarchy, rural talk, and country music, wearing cowboy boots and jeans, driving a fancy pickup truck or fast car, and being able to get out of trouble (Ching \& Creed, 1997; Hemphill, 1974) are elements of the GOB code. The sense of rebellion inherent in each of these items can be easily analyzed.

Cowboy boots and jeans, though socially acceptable today in most regions, were not considered respectable dress for cultured persons until relatively recently. Levis (jeans) born of necessity from a tent material, were used by country farmers who could afford little else, evolved into a statement of rebellion by teenage hoods, and were ultimately adopted by rural Southerners as their dress of choice (Ching \& Creed, 1997; Hemphill, 1974)--due in part to the very fact that they were deemed socially unacceptable by cultured society. Any self-respecting GOB knows to don his cowboy boots and jeans when dressing for a night on the town.

Documented best in the infamous case of the Hatfield-McCoy Feud, the ability to get out of trouble and having a fast car or pickup truck as elements in the GOB code are based in backwoods legend. Even before the Hatfields of West Virginia and the McCoys 
of Kentucky (Hemphill, 1974) became embroiled in their family feud, however, rural Southerners had established an unwritten law about covering for the local "moonshiner" and moonshine runner. With the demise of Southern economic base in the dwindling cotton production, the South had to seek other avenues of income. Because "liquid corn" was much easier to transport and market than grain, bringing in a great deal more money, liquor production grew and "moonshine" became a standard for many rural incomes. "City revenuers" could not collect taxes on the home brew, but in their attempts to destroy the moonshine business, they were held responsible for what was viewed as another unjust encroachment by urbanites and government on a lucrative industry. The illegal brew was marketed by way of the fast car so as to outrun the law, and, thus, was born the "moonshine runner."

Popularized by Hollywood in movies like Still on the Hill and television shows like the Dukes of Hazard, the moonshine runner was almost always a good old boy with a fast car or pickup who could maneuver the dirt roads and backwoods well enough to escape the sheriff or state police. The community that was supported by the sale of moonshine knew all too well how to cover for the good old boys, the moonshiner and the runner. They considered moonshining as a "God-given right, and took it personally when the feds started hounding them" (Hemphill, 1974, p. 244). Moonshiners or "bootleggers" are regarded as true American folk heroes because, "it took a special breed of man [notice the word man] to make moonshine or fly it over the treacherous backroads into Chattanooga and Atlanta with cops on his tail. Southern-style stock-car racing had, as its early heroes, old boys from the hills, uneducated and daring and in possession of damned good automobiles" (p. 245). 
Fondly remembered today in folk tales, a family feud provides another documentation of the good old boys' ability to "get away with things." A series of events that occurred between the Hatfields of West Virginia and the McCoys of Kentucky (Hemphill, 1974), such as murders, unwed pregnancies, kidnappings, and drunken arguments lasted for 25 years. More than "100 Hatfields and McCoys and their allies-not to mention a considerable number of reasonable innocent bystanders--were killed" ( $p$. 234). For the most part, however, "the law tended to stand back in awe and watch from the sidelines."

\section{Gun Ownership}

The sense of rebellion that rural people feel toward "loftier" powers, that have sought to judge them, to invade them, and to force them into becoming more cultured or urbane, permeates many areas of rural society. One of the most obvious areas is that of gun ownership. Though it is well known that handguns are numerous in the city among street thugs and drug dealers, possession of a gun in the rural South is an act of honor and status. Despite biblical admonitions against violence, rural people take great pride in gun ownership and vehemently support the right to own guns. Ching and Creed (1997, p. 29) find that "it is increasingly important not just to own guns, but to display them and aggressively defend their ownership."

The reasons given for their valuing of guns are varied, but it seems rational to believe that gun ownership in the rural South can be related to the valuing of the long rifle, Daniel Boone, Davy Crockett, and other heroes of the American frontier. Though it is often forgotten, it was the rural South that was the first "Western Frontier" in American history; Kentucky was one of the first entry points from the Cumberland Gap when the 
tamers of the frontier crossed the Appalachian Mountains. The gun took an honored place over the mantle as the European Americans fought to wrestle the new land from nature, from wild animals, and from the Native Americans. Later, the gun was invaluable in gaining independence from France, Spain, and Great Britain, and gun ownership was safeguarded by the Bill of Rights in the Constitution. Perhaps it is not so difficult to understand the historical groundings of the gun ownership tradition. Today, finding support in the Constitution, and in the National Rifle Association, has allowed rural people the opportunity to express their resentment of proposed gun control with rebellious fervor. Ching and Creed (1997) indicate that gun ownership, as well as hunting and fishing, is a prominent Rural Value, at least for the patriarchs. Hemphill (1974, p. 233) vividly describes the gun elements of Hatfield-McCoy feud: "Both clans were well stocked with free-living young men [note the word men] who could drink hard and shoot straight and hate long, and the first time they fired on each other was a marauding bands of irregular troops in the war."

\section{Summary}

Because the issue is so relevant to this study, re-affirmation in a summary of the overlapping, inseparable, analogous elements of rural, southern, conservative religious values and southern, conservative rural values is necessary. Specifically, fundamentalist Christianity, political conservatism, social conservatism, traditionalism, ethnocentricity, anti-urban and anti-intellectual postures, acceptance of "pious poverty," and endorsement of the Good Old Boy code are all characteristics that can be named as elements of both southern Religious Values and southern Rural Values (Coreno, 1998; Hemphill, 1974; Peshkin, 1986). 


\section{Related Theory}

Human behavior can be explained to some degree by theory. In order for a researcher to consider why parents are/are not involved in education and to determine how to help them become more involved, a look at applicable behavior theory might be fruitful. Motivation Theory, Comfort Theory, Exchange Theory, Rational Choice Theory, and Spheres of Influence Theory all have something to offer a discussion on motivation for Parental Involvement motivation because of the particular relevance of Affiliation Theory.

\section{Motivation Theory}

Motivation theorists hold that people are motivated to become involved in groups by needs, referred to by some as psychological or social needs: the need for self-esteem, the need to be valued, the need to accomplish, and the need to affiliate or to join groups.

Maslow (1943) concludes that basic human needs provide the motivation for human behavior, and that those needs exist in hierarchical form: first, the need for food and shelter; then, the need for safety; next, the need for love; fourth, the need for esteem; and, finally, the need for self-actualization. When one level of needs is satisfied, a human is motivated by the unfulfilled, or ungratified, need of the next level.

Mayo (1945) finds in the Hawthorne studies that the needs for love, esteem, and self-actualization are also expressed in the needs of affiliation (belonging to a group of like individuals) and achievement. Additionally, he discovers that if personal goals are in opposition to perceived organization goals, workers produce at lower levels.

Hersey and Blanchard (1993) identify elements in a social system that should be considered as factors in the motivation equation: activities (tasks), interactions (behaviors 
between those involved), and sentiments (feelings people have about their tasks and interactions). The interactions and sentiments have a direct effect on the tasks that are accomplished: "In addition to direction, controls, and rewards and penalties, human relationships are most effectively influenced through ... psychological success, and the process of confirmation" (p. 65). Groups that provide direction, rewards, or confirmation of a person's abilities or thoughts are likely to have highly satisfied members.

Hans Zetterberg (1966) further expands the concept of group membership to include fulfillment of basic needs: he theorizes that people will do things that help maintain their self-esteem and identity--as they view themselves and as their associates see them. In his theory of social motivation, he suggests that public opinion is a high motivator for involvement in organizations. "Preoccupation with approval, recognition, admiration, good will, esteem, love, rank, honor" (p. 128), especially from our "significant others," can motivate people to participate in activities that will maintain perceptions of them that they value. If people fail to achieve or accomplish, the loss (or fear of loss) of status leads to an increased need for affiliation (joining into friendly groups)--“Misery loves company.” Specifically, Zetterberg says, "In religion various signs make visible how close a person is to the sacred (for example, special gifts of tongue or admissions to a graded series of holy rites), and call forth reverence from the community" (p. 132).

Schlechty (1990) concludes that those who wish to motivate stakeholders must recognize the "need to feel that what one does makes a difference" and the "need for affiliation and collegial support and interaction" (p. 88). More emphatically, he states, "Status, repute, dignity, values, and beliefs are much more important motivations to 
maintain or improve quality than money" (p. 108).

In summary, perhaps because of the barriers that become more problematic as a child progresses through the higher levels of education, parents find easier, more comfortable, less intimidating sources for gratification of their social needs. Therefore, parents find other organizations with easier connections for interaction and gratification than involvement in their child's education in order to feel fulfilled. Parental disengagement can compound and intensify in direct response to negative school experiences: whether real or perceived, whether encountered as children or as adults. If the tension created by social needs is satisfied through participation, accomplishment, recognition, and affiliation with groups other than school groups, the motivation to become involved in the school can be completely eliminated. However, a parent's involvement with religion or religious groups and the resulting correlation to their involvement with education is one factor in the social gratification-parent involvement equation that seems to have escaped a great deal of investigation. Hence, the studies of the affiliation patterns in other groups need to be examined.

\section{Social Gratification/Deprivation:}

\section{Comfort Hypothesis}

Some researchers have considered the "Comfort Hypothesis" as a major factor in affiliation with social organizations other than schools. A study (Glock, Ringer, \& Babbie, 1967), seeking to find what caused different levels of participation in church by American Episcopalians, established the "Comfort Hypothesis." It was discovered that parishioners, who were deprived of social gratification, satisfaction, and fulfillment at the secular level, turned to the church for substitute gratification and comfort. In every case, 
the parishioners who were considered "secularly deprived" scored higher on the religiosity (degree of religious involvement) scale: "single, childless, old, and lower-class female parishioners scored more than three times as high on the measure of church involvement than did young, married, upper-class fathers" (p. 53).

The Comfort Hypothesis is supported by data gathered from a survey by the University of Michigan Institute of Social Research (Asa, 1995). Christians, who were participating non-members of a church, tended to be unmarried and spouseless. "The unmarried non-member was one-third more likely to be actively involved than the comparable respondent with a spouse and the same applied to non-members without children" (p. 31).

Being married with children is socially more acceptable than being single and childless. An individual may seek to compensate for the lack of secular, social acceptance through church participation. In fact, a sense of social rejection may be "a strong factor influencing deep involvement in the church" (Glock \& Stark, 1965, p. 142). Such need for social compensation might well operate on two levels: not only the social level, but also, the individual or psychological level. First, because the density in rural areas is so low, fewer opportunities exist for social gratification. So the small church satisfies the social need. Second, as has been discussed above, the entire rural area/culture is rejected by much of society. But, usually there is at least one (rural, small, conservative) church that understands, does not reject rural conservative values, and fills the social void.

\section{Exchange Theory}

Taking the Comfort Hypothesis a step further, Bader and DeMaris (1996) identify 
elements of that second dimension of social gratification/deprivation. Based on the Stark-Bainbridge Model, their work finds that not only do people seek what they perceive as reward (social acceptance), but they also seek to avoid what they perceive as individual costs or punishments (rejection). If they are unable to obtain perceived rewards, they will accept other elements as compensation--thus, the name Exchange Theory. For example, if people are rejected by secular society because they are spouseless and childless, they will seek compensation: acceptance in religious affiliation or their "surrogate church family."

However, the Exchange Theory also holds that because humans desire rewards more than they desire compensation, they will be willing to exchange their secondary compensation (church family acceptance) if the individual, psychological, secular reward suddenly becomes available to them (marriage, children, money). A study of Hispanic youth seems to confirm that aspect of the Exchange Theory. It was found (Asa, 1995) that assimilation into secular culture appeared to decrease the religious commitment of the youth.

Conflict arises in the data, though, when socioeconomic status (SES) is considered. Status is commonly recognized as a powerful motivator, a very attractive reward for most people. Especially in American society, the higher one's income is, usually the higher the education level is, and the more socially acceptable he/she is (and the more liberally Protestant the individual's position tends to be). The lower one's income is, the lower the educational level is, the less power he/she seems to have (and the more conservative religious he/she tends to be). However, regardless of its long-accepted position as a social reward, high SES is not necessarily an indicator of church 
participation or membership.

Through the lens of the Exchange Theory, it would appear that low SES would be a negative social factor (generating rejection) that would encourage intensified pursuit of religious affiliation--higher degree of religiosity. Some data (Asa, 1995; Davis \& Robinson, 1996) confirm that assumption. On the other hand, other data (Asa, 1995; Glock \& Stark, 1965) suggest quite the opposite to be true in some cases. "In contrast with the general public, church members are an extraordinarily affluent group" (Asa, 1995, p. 98), and the more economically advantaged a person is, "the more conservative his [church] is likely to be" (Glock \& Stark, p. 133). Perhaps a look at the differing forms of conservatism would help clarify the issue.

\section{Conservatism}

While it is true that the poor tend to be more highly religious or to maintain a higher degree of religiosity, and even though social conservatism is also an indicator of high religiosity, SES and social conservatism are not the only elements of religiosity nor are they mutually inclusive. In addition, financial conservatives (wealthy Rockefeller Republicans, stock market brokers, high society types) are associated with the conservatives in the Methodist, Presbyterian, Episcopalian, and Lutheran denominations. Social conservatives (poor rural farmers, hourly factory workers, commoners, religious fundamentalist/evangelicals) are more closely associated with the Pentecostal, Southern Baptist, Holiness, and Assembly of God denominations.

To illustrate, the well-to-do are often considered to be more economically conservative, but the well-to-do are not automatically more religiously conservative, neither are they more religious nor do they necessarily exhibit a higher degree of 
religiosity. An example found in one study (Asa, 1995) of liberal Methodist, Congregationalist, and Episcopalian denominations demonstrates that these congregations often have higher SES but they tend to take part in fewer church activities. Conservative denominations (Southern Baptist) tend to have congregations who are involved more often in church activities exclusively, less often becoming involved in outside social activities. But, as has been shown, this is not always the case.

Consequently other options must be considered for the impetus to be involved with religious groups as opposed to social or civic groups.

\section{Rational Choice Theory}

Other research into what causes one to affiliate with a liberal, moderate, or conservative denomination examines religion as market goods based on social relationships (Sherkat, 1997). Rational Choice Theory found in that research suggests that individuals make religious choices in consideration of "supernatural compensators (rewards in the afterlife, release from suffering, experience of total inner-bliss)" (p. 68) and in response to adaptive preferences (the more/longer one consumes item X, the more one prefers item X). Consumers, according to Sherkat, also make choices they think will make loved ones happy (sympathy), while avoiding choices they fear will make loved ones sad (antipathy). Choosing the same ideals and beliefs that one's family has embraced is considered to be a "sympathy choice" or a choice made in desire to choose what would make loved ones happy.

Another element of Rational Choice Theory that may be counter to adaptive preference is "seduction," the influence exerted on a person by forces different from his/her family of origin. Relying on previous research, Sherkat (1997) suggests, 
"Fundamentalists' objections to public school curricula rests on this seduction process: once their children have been forced to engage modernist thinking in the classroom, they may come to prefer it. Hence ... education is actively opposed by fundamentalist writers" (p. 70). Educational achievement of fundamentalist children may therefore be hindered.

According to Rational Choice Theory, choices are made, not so much on individual preferences, but more in the social contexts of the religious markets. For example, because social benefits "are only available to African Americans through participation in religious institutions (especially in the rural South), African Americans must attend a church to get the benefits" (p. 75).

\section{Overlapping Spheres of Influence}

Within the realm of the public school community, social affiliation is not necessarily voluntary. To some degree, families are required to be involved in school as long as the children are school age. Participation in parent/teacher conferences, meetings with the principal, sessions with the school counselor may be somewhat forced. Affiliation with school groups rarely fills social needs in this way. One education scholar, Joyce Epstein (1995), has studied the parent-school affiliation in depth over a long period of time. She advances the theory of Overlapping Spheres of Influence. That theory emphasizes the interaction and the interpersonal relationships that influence choices made toward students' learning and development: the spheres of family, school, and community. This theoretical model also places the student in the center--establishing the fourth, but most important sphere. The more the family, the school, the community, and the student communicate and work together, the more "children experience learning 
communities or caring communities" (p. 702). Consequently, the more children will progress in their educational achievement. Epstein states, "As support from school, family, and community accumulates, significantly more students feel secure and cared for, understand the goals of education, work to achieve to their full potential, build positive attitudes and school behaviors" (p. 703). If parents believe in the need of Parental Involvement, they will be encouraged to affiliate without the element of force.

In addition to the four spheres that Epstein (1995) identifies, an often-overlooked sphere of influence is the church community. While the church community sphere of influence can be more important to parents than the school (especially in the rural South), it is too often left out of improvement models. Perhaps because of the trickle-down interpretations of the Constitution concerning separation of church and state, religious affiliation is perchance too often considered irrelevant or invasive to the discussions about student outcomes. Possibly as a sift-down result of administrative judgments concerning separation of religion and schools, the extensive and deep relationship of the rural community to their religious affiliation is perhaps too often ignored when school curricula are constructed.

\section{Affiliation Theory}

In light of such apparent omission, intentional or not, further discussion is needed; for, if the church may help fill a person's needs for love and self-actualization (Maslow, 1943), if it may assist an individual in her/his quest for social acceptance (Mayo, 1945), if it may grant a group of worshippers needed recognition, rank, and honor (Zetterberg, 1966), if it may allow comfort (Glock \& Stark, 1965) to those who are denied and rejected by society, if it may accord status and dignity to those who have been the 
subjects of secular ridicule (Schlechty, 1990), if it may provide earthly compensation for secular rewards that have been withheld from the underclass (Bader \& Demaris, 1996), if it may provide a way for families to bond (Sherkat, 1997), and if it may promise supernatural rewards for those suffering from secular injuries (Sherkat, 1997), the church may surely provide a way to increase the involvement of parents in students' education. Ironically, in spite of a mass of supporting data, leaders in public education have not recognized religiosity as an entity worthy of consideration. It has not been studied as a major sphere of influence in the public education of American children.

Though the broad area of motivation for religious choice in the rural community is an area of much needed research, the basic motivation for a parent's religious choice is important to Parental Involvement. Understanding this religious-school nexus would allow the researcher another descriptive indicator of the culture under study; that indicator could suggest additional areas for further study in the Parent InvolvementReligious affiliation question.

\section{Affiliation Bond}

It is relatively easy to understand how the church, as a Primary Affiliation (PAFF) group, could influence a parent's involvement in school, if the school is not a PAFF group. But another measure is offered as a more accurate indicator of the influence religion has on a parent's behavior. Typically a person must have individual needs and social needs met. But, the ratio of his/her Individual/Psychological/Personal (IPP) needs to his/her Social/Interactive/Cultural (SIC) needs varies. In addition, a group may be chosen to fulfill IPP or SIC needs, depending on how closely a person chooses to bond with that group. The group that a person chooses to meet most of his/her SIC needs is the 
Primary Affiliation (PAFF) group. The intensity or degree that characterizes the bond to that group, usually through fulfillment of IPP needs, has been identified as a critical element of the parental values/religious values/parent involvement relationship and has been designated by the author as the Primary Affiliation Bond (ABOND).

Foundation for the author's proposal is work by Asa (1995): "Religious commitment was related to primary group relations and the degree of acculturation or assimilation they had experienced" (p. 4). For example, the difference in the data might be generated by the "extent to which the church serves as a primary group for any individual member" (pp. 164-165) and the "extent to which persons limit their organizational participation to the church" (p. 168). If the member goes to church only for worship service, but not for interpersonal relationships, then the church likely serves as a secondary group that is one of many outlets for organizational activity. If the member develops overlapping layers of interpersonal relationships in church and social contexts, then the church probably serves as a primary group that is the only real outlet for organizational activity--almost an extension of family or a surrogate family.

It may also be noted that as SES rises and jobs are more professional, then social needs are more often met at the work place. But in rural areas where farming and other agricultural related jobs are more numerous, isolation, separation, and working alone are common experiences. The church is much more likely to be the only available organization other than the school through which affiliation needs can be met, for the SIC as well as the IPP. Though some studies disagree (Hoge \& Carroll, 1978), much of the evidence (Asa, 1995) suggests that the more the church serves to fulfill SIC needs, as well as to fulfill IPP needs, the more likely the church will serve as one's PAFF group, 
and the more likely the group will receive high ABOND scores.

One reason for high PAFF scores for the church by rural parents is the lack of social groups operating in rural, lower SES areas. There are fewer organized offerings for cultural events, fewer organizations, fewer civic groups, and fewer social groups. Therefore, church groups are much more likely to be unavoidable in a person's attempt to meet his/her SIC and IPP needs.

Consistent with this line of thinking is the relatively recent phenomenon of the "superchurch." These superchurches may have congregations of 10,000 or more and often provide entertainment, shopping, school, daycare, and church combined in an interconnected set of social, economic, academic, religious, and personal structures. In effect these superchurches more and more provide for not only Individual/Psychological/ Personal needs and Social/Interactive Cultural needs, but also economic and other needs as well in a meta-community. (In many respects the electronic ministry provides similar aspects of community, e.g., Kenneth Copeland or Trinity Broadcast Network.)

Illustrated in Table 1 are the opposite ends of characteristic continuum lines that Asa (1995) offers as elements of the church, serving as PAFF group (Table 1 was constructed by the author). In this schematic, as the Primary Affiliation (PAFF) group, the church serves for the most part as the only outlet for a person's social activities, a person's best friends are chosen from the church body exclusively, membership in the church is more participatory than observational, and church ABOND is intensified. From this standpoint, a person need look no further than the church for any activities outside of eating and sleeping. To the contrary, if the church serves as only one of many outlets for activities, if best friends come from many different areas of life, and if church provides a 
place to sit and listen but then leave, the church could very well be considered a secondary affiliation group.

Table 1

Characteristics of Church as Primary Affiliation Group Versus Church as Secondary Affiliation Group

Primary

Secondary

Church is the only

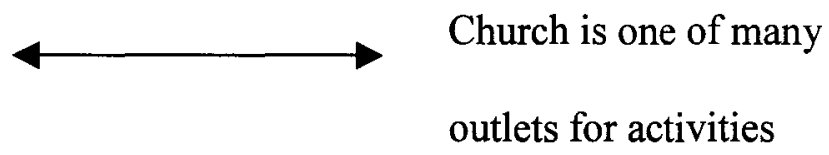

outlet for activities

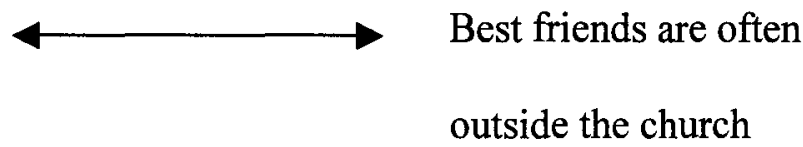

members

outside the church

Membership is more

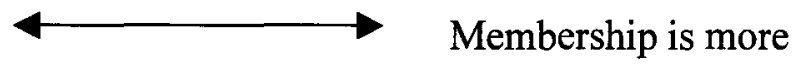

participation

observation

Note. The arrows represent a continuum of positions from one pole to the other.

Identification of parents' PAFF group and measurement of parents' ABOND scores, along with corresponding religiosity scores (PR), may establish the church as a long overlooked, primary stakeholder in public school improvement. It may provide a window for viewing the power of that sphere of influence to affect increased Parental Involvement and, concomitantly, improved Student Outcomes.

Bader and DeMaris (1996) propose four elements as psychological aspects to help determine PAFF and ABOND: attachments, investments, involvement, and beliefs. 
Attachments are strong relationships that tend to cause people to want to act in ways acceptable to the group. If a person has many attachments within the group, his/her behavior will be more consistent with the group's standards. (For example, if a person's mother, two sisters, an aunt, and a cousin attend the same church, his/her attachments will be strong, and his/her behavior will be more according to group standards.)

The greater the investments (or the time, energy, and resources a person places in a group) an individual has, the less willing he/she will be to risk behavior that could threaten his/her bond with the group. If the group is a church body, the more religious the behavior or degree of religiosity a person will exhibit.

The more activities or involvement a person engages in with the group, the stronger the social bond and the less likely his/her behavior will deviate from the most conservative (for that particular group) stance. The longer a person does $\mathrm{X}$, the more likely a person will continue to do X. (The longer an individual speaks in tongues, the more likely the individual will continue to speak in tongues and participate in the church that believes in speaking in tongues.)

Finally, the stronger a person's support of affirmed beliefs, the weaker his/her chances of being compelled to act outside the doctrines of the group. (For example, if a member has supported the Southern Baptist's rejection of women as pastors for many years, that person will not be easily persuaded to change his/her mind to vote to allow a woman to pastor the church--regardless of how strongly he/she hold's women's rights in other areas.)

\section{Summary}

According to the review of literature, Parent Involvement is necessary for students 
to achieve at the highest levels in school. Perhaps because of the barriers that become more problematic as a child progresses through the higher levels of education, parents find easier, more comfortable, less intimidating sources for gratification of their social needs. Parental disengagement can compound and intensify in direct response to negative school experiences: whether real or perceived, whether encountered as children or as adults. Additionally, if the tension created by social needs is satisfied through participation, accomplishment, recognition, and affiliation with groups other than school groups, the motivation to become involved in the school can be completely eliminated. Similarly, the literature reveals that in southern rural communities the elements that may impact Parental Involvement (PI) include Parent Religiosity (PR), parent Rural Values (RV), and Primary Affiliation (PAFF) and Affiliation Bond (ABOND).

Despite these findings, only a few studies have been designed to determine the effect of religiosity on Student Outcomes (SO) as mediated by PI. Moreover, most of the elements of PI and SO have been investigated as they exist independently; the intersection of these elements, as they overlap, combine, and exert influence over the other elements to impact SO, has not been studied.

In summary, the literature has shown that for student outcomes to improve students should be encouraged and led to perform better; for students to be led to perform better, parents need to be become more involved. It would seem to follow that for parents to become more involved in their children's education, parents would need to have Social/Interactive/Cultural (SIC) and Individual/Personal/Psychological (IPP) needs met through school involvement. The literature recognizes PR, RV, PAFF, ABOND as elements of the environment, as constructs for this study. Researchers in the area of PR 
have cited that construct as interfering with positive $\mathrm{PI}$ as well as hindering SO (especially those of non-compliant female children of conservative religious denomination parents). Available literature suggests that PI decreases as the degree of conservatism/evangelicalism of the religious denomination increases. Rural-ness (RV) has been shown to reflect a higher degree of conservatism/religiosity (PR). In addition, the literature reveals that RV tends to give rise to anti-intellectual attitudes (AI) and fear in parents of anything urban in nature, including urban-reformed education. Particularly affected is the rural South.

Also if personal (IPP) and social affiliation (SIC) needs are met through religious connections, parents may not feel the need to become involved in the school. Inversely, if the schools do not actively seek to fulfill personal and social affiliation needs of parents, they may increasingly search for fulfillment through religious means, thereby decreasing their tendency to become involved in school activities. If their needs are met by validation, accomplishment, collegial support, and interaction derived through membership in other organizations (social, civic, or church), there may be no deficit of social needs, and no resulting motivation to become involved in their child's school. Further, because of the potentially hostile environment that may be associated with previous or current negative school experiences, PI motivation could indeed be nullified through perceived social rejection by the school environment. For PI to increase, school communities may have to respond to the caveats issued by comfort theorists who have studied affiliation needs.

This study has been built on the shoulders of the researchers before. It has been well established that parental values affect the degree and type of PI within any given 
community. However, in most previous studies, the constructs have been studied only independently of each other. The intersection of these constructs, as they may affect student outcomes (PI, RV, PR, PAFF and ABOND) has not been previously investigated in a single study. Assessing the combined effect of these constructs is the foundation of this study. 


\section{CHAPTER III}

\section{METHODOLOGY}

The purpose of this study is to examine the interrelationships of Conservative Christian Parent Religiosity (PR) and parental Rural Values (RV), as mediated by Parental Involvement (PI) and controlling for Demographics (D), to Student Outcomes (SO) in a Southern rural middle school. This is an exploratory case study of mixed design that uses quantitative and qualitative methodology: surveys, interviews, student grade point averages, document analysis, and researcher field notes.

Quantitative methodology is utilized to analyze responses to survey instruments. Those instruments are designed to determine type and degree of Parental Involvement (PI), degree of Parental Religiosity (PR) and Rural Values (RV) as they relate to education, Demographics (SES, special education programs, and Family Structure), and Student Outcomes (SO). Supplemental qualitative methodology from interviews, document analysis, and researcher field notes (RFN) are used to provide additional data for analysis.

Babbie (1990) maintains that survey research allows generalization from a sample to inference for an entire population. However, because the $N$ in this case study is so small, because the sample represents a small population, because only one school is under study, and because the researcher is an observer and a participant, such generalization must be done with extreme caution, if at all. However, this is an 
To compensate for these problems, supplemental qualitative data are collected to provide a rich texture for the quantitative analysis. These data come primarily from interviews of selected individuals who make up the different role groups at Dixon Elementary/Middle School. In addition, during 1999-2001, Researcher Field Notes (RFN) about the school, the curriculum, the faculty, the students, the Parent Involvement programs, and other elements were gathered. Those RFN provide further triangulation of data.

The case study allows for a more complete picture of the setting through qualitative triangulation. Miles and Huberman (1994) maintain, "Qualitative research is conducted through an intense and/or prolonged contact with a 'field' or life situation," (p. 6). The researcher's life-long association with the field under study provides that intense, prolonged contact. Through her association with the rural culture of the Dixon community, she is able to "explicate the ways people ... come to understand, account for, take action, and otherwise manage their day-to-day situation" (p. 7). Researcher Field Notes (RFN) are analyzed in order to assemble them into "semiotic segments" that "can be organized to permit the researcher to contrast, compare, analyze, and bestow patterns upon them" (Miles \& Huberman, p. 7). Descriptive data of the school, the curriculum, the faculty, the students, the parent involvement programs, and other elements are provided through researcher observation.

\section{Sources of Data}

To capture the best descriptions of Dixon Elementary Parental Involvement, Parental Religiosity, parental Rural Values, and Student Outcomes, several sources are tapped. All of the 109 students of DEMS and their parents are asked to complete and 
return surveys. Members of key role groups are interviewed to provide additional perspective. These include ten students and parents of DEMS, one of the teachers of DEMS, one parent and student of a nearby Christian school, one parent and student who has withdrawn from DEMS to home school, the custodian who works in the middle school, and a pastor of a local Baptist church who serves as a school bus driver. Student Outcomes are gathered from records at the central office and the high school guidance office, and Researcher Field Notes provide supplemental information.

\section{Students and Parents}

Subjects for this study are the 109 seventh and eighth grade students at DEMS and their parents (during 1999-2001). For the ease of discussion and clarity, "parent" is considered to be a person assuming the primary role of caretaker. While "caretakers" may be a better word for people assuming the role of parent--many students of DEMS are cared for by other adults such as aunts, uncles, older siblings, grandparents, and live-in adults--most caretakers would more readily understand and accept the title of "parent."

Students are divided into two classes of seventh graders and two classes of eighth graders. They are between the ages of twelve and fifteen. Their academic attainment levels, socio-economic levels, GPAs, parent education levels, parent employment, and religious affiliations vary widely; however, the ethnicity of the students is $98 \%$ European American.

Most students are "average" achievers who report seeing little point in working for high grades when average grades will allow them to participate in sports. Truancy is often a problem with some students, and parents of truant students often reveal no concern about their children's absences until court workers get involved. Overall 
lackadaisical attitude is reflected among the students that mirrors the anti-intellectual posture proffered by Hofstadter (1963). The middle school faculty frequently raises questions about these attitudes as a cause for concern in discussions (RFN).

Parents of students at DEMS are typically lower-middle to middle class. Most have a high school education and express conservative religious and rural values. They rarely attend Parent/Teacher Organization meetings and have almost no working knowledge of School Based Decision Making. Only $10-15 \%$ of the parents typically attend the Parent/Teacher Conferences that are scheduled twice a year; the parents that do attend are usually the parents of higher achievers. Over the twenty-plus years as a teacher at DEMS, the author has been told by many parents that the school is unwelcoming and cold. The parents have expressed a sense of rejection from the faculty and the principal. However, students who are involved in sports programs tend to have parents who are more involved, perhaps because the school itself does not sponsor sports programs at DEMS. Basketball, football, cheerleader squads, and drill teams are coordinated, coached, and supervised by parents outside the domain of school authority.

Parent selection for the interviews used a rating matrix created by the author. The matrix allows for high/middle/low contrasts on the two dimensions of Parent Involvement and student performance. The subjects selected represent six of the nine possibilities: high-parent/high-student $(\mathrm{HH})$, middle parent-high student $(\mathrm{MH})$, low parent-middle student (LM), middle parent-middle student (MM), low-parent/middle student (LM), and low-parent/low student (LL), all drawn from the cells generated by the matrix of Parent Involvement crossed by Student Achievement. Categories were further differentiated according to seventh or eighth grade, e.g. 7HH. In addition, two other 
designations provided additional perspective: a parent severed as a substitute teacher in the school, denoted by a small $s$ (7HHs), and a student withdrew from school to be home schooled, denoted by a small $h(8 \mathrm{HHh})$.

The score categories for the matrix are based on the following schema: Each parent receives one point for his/her presence at the October 21, 1999, Parent-Teacher Conference. Non-attending parents receive a zero. Parents who were present for a field trip receive one point for each of the two field trips. Participating parents also receive as many as three points based on RFN records of PI-as (active at school.) (The parent chosen for scoring from a home not sending a representative to the PT conference is selected from the parents who responded to the Parent Survey.) Total possible points awarded are six. Parents are then placed into low, medium, and high groups based on the interval scale developed from the points $(1-2=$ low; $3-4=$ medium; $5-6=$ high $)$. The scores derived from this matrix are used for qualitative description only. (See Appendix I for further description of selection matrix.)

To establish the student achievement scores for the interview selection matrix, points are given for student scores of $75+$ percentile on KCCT or KIRIS core content areas, for GPA of 80-100 in core content areas, for student activities and student leadership roles listed on the student survey, and for RFN data collected by the researcher. Student achievement points possible total 18. Divided into three groups of six points each, the resulting interval scale establishes the low, medium, high groups of students: $1-6=$ low; 7-12 $=$ medium; 13-18 $=$ high. (See Appendix I for additional information.) The student scores derived from this matrix are used for qualitative description only. 
The $3 \times 3$ matrix $(H, M, L)$ generates the nine parent-student groups, coded as pairs of letters. The first letter of each pair represents the parent while the second letter represents the student, from HH to LL. (See Appendix I.) It should be noted again, this matrix is for interview selection and qualitative description only.

\section{Teacher at DEMS}

A subject for the interview was selected according to several considerations. First, he has resided in the Dixon community (for 20+ years). Second, he has the longest tenure at Dixon Elementary/Middle School (20+ years). Third, he is the only teacher who agreed to be interviewed. Qualitative descriptions obtained during a teacher interview provide triangulation of survey data.

\section{Parents and Students of Christian School and/or Home School}

Interviews are conducted with parents/students who have withdrawn from public school to attend a conservative Christian school in a nearby town or who have left the conservative Christian school to return to the public school. A parent who withdrew children for home schooling is also interviewed. Parents/students are interviewed upon a voluntary basis.

Individuals who have withdrawn for home schooling may tend to belong to more conservative churches and may be more vocal about their religious beliefs. Some conservative home-schooling parents rallied forces to have the Ten Commandments displayed in the school. When that did not materialize, they created tee shirts that displayed the Ten Commandments and gave them away to every student to wear to school. Eventually, some of these parents withdrew their children from DEMS. Some of these parents objected to the teaching of Greek mythology and evolution. 


\section{Pastor of Local Baptist Church}

Because the pastor of the local Baptist Church has a congregation that includes many families with children in the middle school at Dixon, and because the pastor is also a substitute bus driver for the school system, he represents a unique blending of community and school staff perspectives.

He moved to Dixon from Louisville about five years ago and lived in a home just outside of town. He frequently drove the bus for the Dixon routes and was highly interactive with students and parents on his route. He also interacted with the staff at DEMS. His impressions of the religious values of DEMS parents, the rural values of the students and parents, and of the school environment are drawn from his close connection and interaction with the subjects. His revelations provide supplemental data from an insider-outsider perspective of the DEMS community.

\section{DEMS Custodian}

The building has three main custodians. Each works a different "school" within the building. The custodian in charge of the middle school has had two children go through DEMS a few years ago. Thus her perceptions of student and parental values and of school environment reflect both parent and school staff roles.

\section{Researcher-Participant}

As a former teacher at DEMS, the researcher serves as a participant-observer in this case study. Though that role presents cause for concern about researcher bias, the role also provides the researcher with a unique familiarity and understanding of the community under study (see below, Participant-observer bias). Researcher Field Notes collected during the years of 1999,2000 , and 2001 are analyzed for supplemental 
information and triangulation of data.

\section{Student Achievement Data}

Student Outcome data are archived at the central office and in the high school guidance office. Records of GPA are available to the author through parent release only. The parent release is included with the parent survey and has been cleared as a part of human subjects oversight (see Human Subjects Review Board, below).

\section{Procedures}

Procedures for the study were carefully planned because of the nature of the study and because the researcher is no longer active in the school system, which limits her access to data. At the beginning of the study, it was expected that $95-100 \%$ of surveys would be returned. Now that the author is no longer in the classroom, return of surveys was much more difficult. In addition, at the beginning of the study, access to student records would have been automatically granted to the researcher as a teacher in the system because of the academic nature of this study. Now, access to student records was achieved only though signed parental permission. Also, confidentiality of records is a major concern, addressed through the consent form as a part of the Human Subjects Review Board protocol.

\section{Parents and Students}

The researcher was allowed to conduct surveys and interviews of the seventh and eighth grade students and parents on a voluntary basis and in a manner "not to interfere with instructional time," as required by the superintendent and principal. Hence, the surveys and interviews are conducted through mailings, email, telephone communications, and after-school sessions. (See Appendix J for permission to conduct 
study, Appendix $\mathrm{K}$ for adult consent form, Appendix L for parental consent form for child survey participation, and Appendix $\mathrm{M}$ for parental consent form for child interview participation.)

The parents of DEMS students are selected for interview based upon a combination of several identifiers. Their responses or non-responses to surveys, their attendance or non-attendance at the Parent-Teacher Conference (October 21, 1999), and their students' outcomes are used to form a selection matrix from which potential interviewees are chosen. (See Appendix I.)

The researcher contacted parents and students of DEMS and those of the Christian school or the home school by phone or email to request interviews. Permission forms for the student interview were signed by the parent (Appendix M). Interviews were done in person when possible. The phone and email provide alternative methods for gathering information. The researcher interviewed four parents and students from the eighth grade and six parents and students at the seventh grade.

Interviews are a direct method of obtaining qualitative data to provide a more complete picture of the case study. The parent interview instruments and the student interview instruments are parallel in content to the survey instruments so that comparison to quantitative data may be accomplished. When possible, interview questions were given to the subjects several days before the actual interview to allow for adequate consideration of the questions. Advance reading may help to quell the sense of threat that is often connected to the "unknown questions." Interview sessions lasted no longer than one hour and were conducted at a site agreeable to the subjects.

The use of a tape recorder was determined in advance as directed by the subjects. 
Although the tape-recorded interview makes retrieval and storage of information easier, it may also inhibit responses. Reluctant subjects' wishes regarding recording were honored. Researcher observations are recorded following the interview in all cases, as supplemental context to the interview responses.

Interview narratives were analyzed for common constructs, patterns, trends, and anomalies as guided by the Research Questions. All transcriptions, tapes or notes are kept in a locked file (see Marshall \& Rossman, 1995) in the researcher's office.

Consistent with work by Dillman (1978), the survey has been prepared administered using the Total Design Method. The surveys were developed over the course of a year and involved several checks to ensure validity (see Validation Process and Validity sections, below). Student Outcome data and identification codes were added to the surveys before mailing so that the correct student/parent pair might be identified and so that follow-up requests for survey completion might be issued. Then the surveys were sent to subjects by mail with a cover letter (Appendix $\mathrm{N}$ ) and with instructions and directions to return before a designated date. In order to lessen the opportunity for social desirability bias, the survey was self-administered (Fowler, 1993). Self-administration encourages respondents to answer more honestly because confidentiality is more likely assured. Sensitive areas of religion, SES, and student accountability necessitate confidentiality; therefore, a self-addressed, stamped, return envelope was provided for return of the survey with the likelihood of enhancing the return rate.

Student surveys were included with the parent surveys as they were mailed to the parents. They were likewise self-administered to allow for the greatest degree of nonbias and confidentiality. The students were instructed to return their surveys along with 
the parent surveys in the return envelope provided to them.

A cover letter prepared on university letterhead explained the intent and the design of the instrument; the surveys were pre-coded so that parent/student/outcome relationships might be linked. University sponsorship has been shown to significantly increase response rates compared to the use of a commercial sponsor (Faria \& Dickinson, 1996).

Because low response rates have been noted as one of the major weaknesses of survey research (Pinsonneault \& Kraemer, 1993), after one week, after two weeks, and after four weeks following the mailing, the researcher used the telephone and email to remind and encourage the return of surveys. A dollar bill (Borg \& Gall, 1989) was attached to the parent's survey to increase the likelihood of return. Previous research (A. Church, 1993; Hansen, 1980) has shown that respondents feel obligated to respond and that response rates are increased when modest incentives are used because respondents view themselves as contributing to a worthy cause.

Checks with five non-respondents by telephone were done to guard against the possibility of their potential answers being systematically different from the returned surveys, thereby biasing the results.

\section{Teacher}

The teacher at DEMS was contacted by telephone. He was interviewed at a site chosen by him to provide as much comfort and validity as possible. This teacher also has a son who was a participant in the study, thus representing the dual perspectives of parent and teacher. 
The local pastor, who also served as a substitute bus driver, was interviewed at the church. He was readily available during the daytime in his office. He was contacted in person.

\section{Other Parents and Students}

Parents and students who have withdrawn from DEMS to attend the local Christian Heritage Academy were contacted by phone. They were interviewed by telephone because that was most agreeable to them. Follow-ups were conducted by email. Those withdrawing to home school chose to be interviewed in their home.

\section{Student Achievement Data}

Data for Student Outcomes were gathered from files stored at the central office and the high school guidance office. A parent release form is included in the survey packet. If a parent signed the release form, the researcher was permitted access to the data from the office of the assistant superintendent and from the high school guidance counselor. That information was then coded according to respondent and added to the aggregate database for the study.

\section{Research Questions}

Research questions, based upon the conceptual model presented in Figure 1, provided the foundation for construction of the instruments used in this study. The questions and Figure 1 are repeated below for the benefit of the reader. They indicate possible relationships between the variables and their overlapping and/or mediating spheres. The numbered arrows correspond to the following Research Questions:

1. How do Demographic factors relate to Student Outcomes?

2. How do Demographic factors relate to Parent Values:

a. Rural Values? 

b. Parent Religiosity?

3. How do Demographic factors relate to Parent Involvement?

4. How do Parent Values:

a. Rural Values

b. Parent Religiosity

relate to Student Outcomes?

5. How do Parent Values:

a. Rural Values

b. Parent Religiosity

relate to Parent Involvement?

6. How does Parent Involvement relate to Student Outcomes?

7. How do Parent Values:

a. Rural Values

b. Parent Religiosity

relate to Student Outcomes, controlling for Demographic factors?

8. How does Parent Involvement relate to Student Outcomes, controlling for Demographic factors?

9. How do Parent Values:

a. Rural Values

b. Parent Religiosity

relate to Student Outcomes, as mediated by Parent Involvement?

10. How do Parent Values:

a. Rural Values

b. Parent Religiosity

relate to Student Outcomes, controlling for Demographic factors, as mediated by Parent Involvement? 
Independent Variables

Dependent Variable

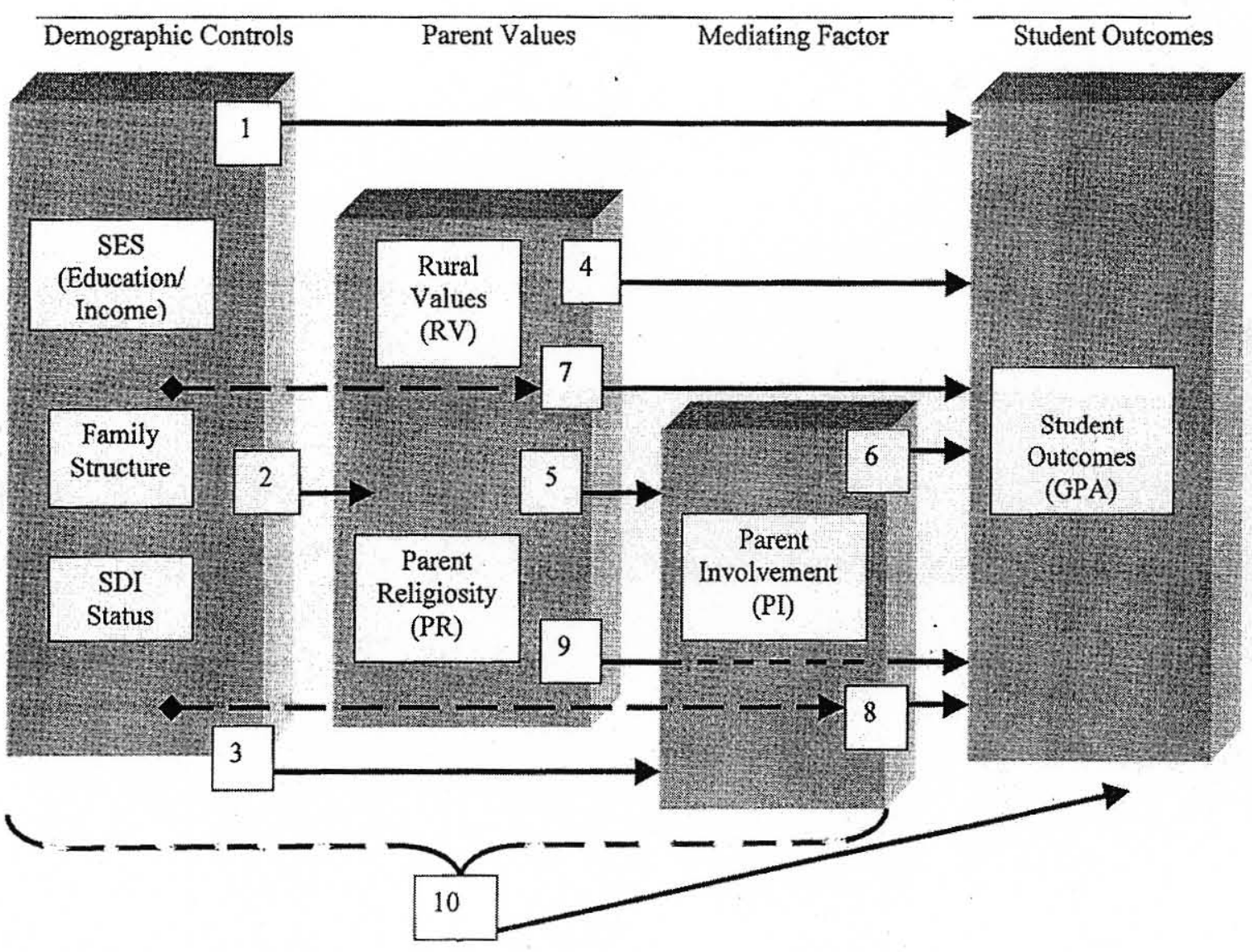

Figure 1. The relationship of Parental Rural Values and Parent Religiosity to Student Outcomes as mediated by Parent Involvement and controlled for Demographics. 


\section{Instrumentation and Variables}

Exhaustive literature review netted no instrument that was precisely focused on and reflective of the specific, overlapping areas in question as they pertained to the southern, rural, middle school. Though there exist surveys to measure PI, RV, and PR separately, to use these instruments in their entirety would make them prohibitive in time and length. Modifying items on instruments could affect their validity as well as their reliability, especially when used in combination with other instruments. Therefore, because there exists no instrument specific to this case study, and because using partial selections from previously validated surveys would probably alter their reliability anyway, the researcher developed surveys and interviews by drawing ideas from developed instruments that have been validated by previous studies (addressing only the specific areas of PI, RV, and PR as they intersect, overlap, blend, and manifest in the small Southern rural conservative middle school community).

According to many studies (Dornbusch, 1988; Epstein, 1995; Fehrmann, Keith, \& Reiners, 1987; Henderson, 1987; Maxim \& Five, 1997; O’Neil, 1991; Smith \& Martin, 1997; Steinberg, 1996; Tennies, 1986; Useem, 1991), PI is directly related to SO in a school. Therefore, many of the questions on the parent survey concern type and degree of PI. Questions concerning RV and parallel PR elements are structured by reference to works by several authors including Asa (1995), Ching and Creed (1997), DeYoung and Laurence (1995), Fox (1997), Hofstadter (1963), Howley and Howley (1995), Little (1998), Miller (1994), and Theobald and Nachtigal (1995). In addition, other PR survey questions are based on research of numerous other authors such as Darnell and Sherkat (1997), Davis and Robinson (1996), Glock (1973a), Hofstadter (1963), King and Hunt 
(1975), Seal and Harmon (1995), Sherkat and Ellison (1997), Stark and Glock (1968), and Wuthnow (1973).

Construction of approximately 250 items was accomplished after an extensive review of the available literature. Questionnaire items were then selected based on their acceptance by experts in the field as indicative of the characteristic under assessment. In addition to the experts identified above, scholars, and their fields of expertise, whose work provided multiple validation for the items selected, are provided below:

Parent Involvement: Cushman (1993), Epstein (1995), Feldman (1994), Kelly and Pope (1995), and Mead (1992).

Parent Rural Values: Peshkin (1986, 1994).

Parent Religiosity: Bader and DeMaris (1996), Cornwell et al. (1986), Ellison and Sherkat (1993), Perrin (2000), Regnerus (2000), Sherkat (1997), and Sherkat and Ellison (1997).

Depending upon repeated research validation as applicable plus review from the perspective of the Researcher's Field Notes (RFN), the 250 original items were subsequently reduced to a total of 124 content questions. Demographic questions (21) were added to the Parent Survey in sections V A, V B, and V C. (Specific questionnaire items may be viewed in Appendixes $\mathrm{O}$ and $\mathrm{P}$. A scoring rubric and coding schematic for the questionnaire items follow in Appendixes Q and R.)

The actual description of the content of each instrument is incorporated into the section that defines and organizes all variables by category--dependent or independent, and type of independent variable. The definitions of variables are arranged by and consistent with the theoretical model in Figure 1. Explicit operational definitions are attached at Appendix S.

Survey Design 
The Parent Survey has five sections. The first, which is divided into three subsections, I A, I B, and I C, is designed to assess the type and degree of Parent Involvement (PI). Rural Values (RV) is investigated in Section II. Parent ReligiosityBeliefs (PR-BEL) and Parent Religiosity-Experience (PR-EXP) are the subjects of Section III A. Parent Religiosity-Ritual (PR-RIT) is addressed in Section III B. Section IV A is designed to identify Parent Religiosity-Primary Affiliation (PR-PAFF); questions to determine the degree of the Parent Religiosity-Affiliation Bond (PR-ABOND) make up Section IV B. Last, Section V requests responses to Demographic (D) questions such as gencier, number of children, income, and education.

The student survey also has five sections. Sections I through IV are parallel to the parent sections I through IV to allow for comparison of answers. However, Section V requests responses to questions such as student activities and leadership roles.

\section{Classification of Variables}

This study has three types of Independent Variables. They are Demographics, Parent Values, and Mediating Factor. The Dependent Variable is Student Outcomes. Discussion of these variables is ordered according to the conceptual model of the overall study (Figure 1).

The Independent and Dependent Variables for the calculations in a given RQ are not necessarily the same as their classification in Figure 1 for the entire study. Table 2 gives the actual Independent and Dependent Variables for the analysis in each specific question. (See also Appendix for measurement of scales.) 
Independent Variables

\begin{tabular}{|c|c|c|c|c|c|}
\hline $\begin{array}{l}\text { Research } \\
\text { Question }\end{array}$ & Control & $\begin{array}{l}\text { Parent } \\
\text { Values }\end{array}$ & $\begin{array}{l}\text { Mediating } \\
\text { Factor }\end{array}$ & $\begin{array}{l}\text { Dependent } \\
\text { Variable }\end{array}$ & Analysis \\
\hline 1 & Demographics & & & $\begin{array}{l}\text { Student } \\
\text { Outcomes }\end{array}$ & Stepwise \\
\hline 2 & Demographics & & & Parent Values & Stepwise \\
\hline 3 & Demographics & & & $\begin{array}{l}\text { Parent } \\
\text { Involvement }\end{array}$ & Stepwise \\
\hline 4 & & $\begin{array}{l}\text { Parent } \\
\text { Values }\end{array}$ & & $\begin{array}{l}\text { Student } \\
\text { Outcomes }\end{array}$ & Stepwise \\
\hline 5 & & $\begin{array}{l}\text { Parent } \\
\text { Values }\end{array}$ & & $\begin{array}{l}\text { Parent } \\
\text { Involvement }\end{array}$ & Stepwise \\
\hline 6 & & & $\begin{array}{l}\text { Parent } \\
\text { Involvement }\end{array}$ & $\begin{array}{l}\text { Student } \\
\text { Outcomes }\end{array}$ & Stepwise \\
\hline 7 & Demographics & $\begin{array}{l}\text { Parent } \\
\text { Values }\end{array}$ & & $\begin{array}{l}\text { Student } \\
\text { Outcomes }\end{array}$ & Hierarchical \\
\hline 8 & Demographics & & $\begin{array}{l}\text { Parent } \\
\text { Involvement }\end{array}$ & $\begin{array}{l}\text { Student } \\
\text { Outcomes }\end{array}$ & Hierarchical \\
\hline 9 & & $\begin{array}{l}\text { Parent } \\
\text { Values }\end{array}$ & $\begin{array}{l}\text { Parent } \\
\text { Involvement }\end{array}$ & $\begin{array}{l}\text { Student } \\
\text { Outcomes }\end{array}$ & Hierarchical \\
\hline 10 & Demographics & $\begin{array}{l}\text { Parent } \\
\text { Values }\end{array}$ & $\begin{array}{l}\text { Parent } \\
\text { Involvement }\end{array}$ & $\begin{array}{l}\text { Student } \\
\text { Outcomes }\end{array}$ & Hierarchical \\
\hline
\end{tabular}

\section{Independent Variables}

The Independent Variables are discussed below. Included in the discussion are 
the instruments and the scoring and coding of data for each type of variable:

Demographics, Parent Values, and Mediating Factor.

\section{Demographic Controls}

The Demographics (D), or personal characteristics, of a respondent are known to affect the results of individual surveys as well as differences on various outcome measures. In order to investigate these differences, the fifth section of the survey provides this information.

Demographic Constructs

Three Demographic constructs are considered. They are Socioeconomic Status, Family Structure, and Specially Designed Instruction.

Socioeconomic Status (SES). The Socioeconomic Status of a family is determined by elements affecting the financial abilities of a family as well as the education level attained by the family leaders. An abundance of research confirms that students who come from low SES families obtain lower achievement scores (e.g., White, 1982). The SES construct typically includes income and job status, education, and other resources.

Family Structure (FS). Family Structure may well have an impact on the family Demographics. The size of a family, the ratio of caretakers to children, the number of generations represented by the family unit and the availability of transportation to family members represent elements of FS that could affect educational achievement.

Specially Designed Instruction Status (SDI). Specially Designed Instruction (SDI) identifies the help a student receives, in addition to regular classroom instruction, from a specially trained teacher in either a pull-out or a resource setting. At times known as special education, SDI may be initiated if/when a child is classified as having special 
needs: included as special needs are Attention Deficit Disorder, Attention Deficit Hyperactivity Disorder, Learning Disabled, Educable Mental Handicap, Hearing Impaired, and Visually Impaired.

Student involvement in Specially Designed Instruction (SDI) represents an impact on family demographics in several ways. First, because the children have learning problems, SDI status could evidence an extra drain on family economics. Second, in order for students to participate in the special education programs, they are required to have their parents involved. The Parent Involvement scores for these students, therefore, are expected to be higher.

\section{Instrument Items}

Survey Section V A (see Appendix I for full Parent Survey) has nine questions that request a word, a number, or a checked response written into a blank. Items included are such questions as "What is the highest education level you have completed?" and "How many children are in the family?" These questions along with Section V C (estimate of family income) should help establish the SES. Items in Section A, which ask for the number of caretakers, the number of children, and the number of dependent adults living in the home, help establish a FS score.

Students are provided parallel questionnaires that mirror the parent instruments and are coded to connect the child's questionnaire to the parent's. The Student Survey (Appendix K) seeks to elicit student perceptions to items that correspond to the items covered by the Parent Surveys. The Student Survey establishes a parallel Demographic score that may be used for comparison.

The Student Interview question \#13 asks students to tell about "parents' activities 
outside school," and question \#14 asks with whom parents "seem to spend the most time." The Parent Interview includes question \#13: "Tell me about your activities outside school." The answers to these questions add supplemental descriptive information that was used in the interview selection process.

Scoring of Items

Individual scores and indexes for Specially Designed Instruction (SDI), Family Structure (FS), and Socioeconomic Status (SES) provide a basis for examining the relationship between family demographics and other Independent Variables and the Dependent Variables. Because it is generally Family Structure (FS) and Socioeconomic Status (SES), and not the specific sub-components that are important to this study, collapsing of data from Section V results in three scores: SES, FS, and SDI. In order to simplify scoring for the Demographic survey section, a scoring system, built upon onepoint increments, is used to establish the SES and FS scores. (See Appendix Q.) Points are given depending on an ordinal assessment of each family characteristic. The SDI scoring, in contrast, is merely a reflection of participation or non-participation.

The SES score is a result of collapsing education attainment (Section V A item 9) and income level (Section V C items 1-6). There are 6 points possible for education, ranging from 1 point for an eighth grade education to 6 points for graduate school + . Six points are possible for income, ranging from 1 point for $\$ 0$ - 15,000 to 6 for $\$ 75,001$ $90,000+$. The combined SES is based upon education points plus income points. The resulting interval scale ranged from 2-12. (See Appendixes Q and S.)

The FS score results from determining the child-to-caretaker ratio. The ratio is based on items 3,6, and 7 from the V A section of the survey; the ratio represents the 
number of children plus the number of dependent elderly or disabled adults (who require assistance) in the home divided by the number of adult caretakers in the home.

For the SDI score, if a student is receiving SDI assistance, it is noted on the scoring rubric as 0 points. Non-assisted students are designated by a 1 .

\section{Parent Values}

Two types of parental values are expected to correlate to the Dependent Variable: Rural Values (RV) and Parent Religiosity (PR). Section II of the survey applies to Rural Values; it includes 30 items. Sections III A and III B include 22 and 11 items that relate Parent Religiosity values. Also, because the Parent Survey and the Student Survey are parallel in structure, the letter $\mathrm{S}$ is used to identify student coding and for recording data for all constructs. For example, RV-AI is the code for Rural Values-Anti-Intellectualism for student surveys, whereas, RV-AIS is the parallel code for student surveys.

\section{Rural Values Constructs}

An important facet of life in rural America, especially in the South, is rural values. Rural values appear to be reflected primarily in five Rural Value characteristics or Constructs: Anti-Intellectualism, Social Conservatism/Traditionalism, Ethnocentricity/Community-Centrism (Little, 1998; Little \& Miller, 2001), Stewardship of Earth, and Good Old Boy.

Anti-Intellectualism ( $R V-A I)$. In response to many factors, including denigration by sophisticated urbanites, rural populations have developed a well-documented rebellious attitude toward aspects of academic nature. Identified by Batson (1993), Darnell and Sherkat (1997), Gilchriest (1995), Hemphill (1974), Hofstadter (1963), Peshkin (1994), Reed and Reed (1996), Wilson and Ferris (1989), and RFN, and dubbed 
Anti-Intellectualism by Hofstadter in the 1960's, this attitude has evolved into an apparently rather firmly held rural value construct that includes disdain for items designated as "cultural" by urban cosmopolitans: foreign language, literature, art, music, dance. An emphasis on "life learning" or practical, useful skills training and on sports programs is often found to replace emphasis on "cultural" programs. The survey addresses RV-AI with items such as "Rural students need to be taught foreign languages" (reverse scored).

Social Conservatism/Traditionalism (RV-SC/T). Social Conservatism and Traditionalism have been identified as constructs by Batson (1993), Ching and Creed (1997), Gilchriest (1995), Little (1998), Peshkin (1994), Reed and Reed (1996), and Wilson and Ferris (1989). They are so closely related, however, they are combined as one construct in this study (RV-SC/T). (Combining the constructs also allows for elimination of some items to shorten the survey.) $\mathrm{RV}-\mathrm{SC} / \mathrm{T}$ is considered a Rural Value construct because resistance to change tends to be deeply entrenched in rural cultures. In addition, a strong central leader is embraced as necessary despite possible disagreement with that leader's individual philosophy or actions. Strong, central leaders with firm control lend a sense of security to a change-phobic society. Also, rigid adherence to custom and tradition promote a male-domination/preference within the rural community that has been present since the arrival of the Puritans. "I'll support the principal's decision even if I don't agree with it" is a survey item representative of those pertaining to $\mathrm{RV}-\mathrm{SC} / \mathrm{T}$.

Ethnocentricity (RV-E). Ethnocentricity (RV-E) refers to a culture's belief that its place of origin, its way of life is better than anyone else's, and can often include 
overtones of ethnic relations or racism. However, in the current context RV-E represents pride in rural heritage, reflection of rural standards and morals, preference of the rural life for family rearing, and favoring of stories, music, and social activities particular to the rural South. The element, which has been renamed Community-Centrism in Little and Miller (2001), refers more to a community's belief that the particular community's way of life and values are better than anyone else's. In rural communities it may be impossible to distinguish between these constructs. In addition, the distinction between the two is really of small consequence to the outcome of this study; therefore, the constructs are combined and referred to as simply RV-E. RV-E has been noted in other literature such as Batson (1993), Gilchriest (1995), Reed and Reed (1996), and Wilson and Ferris (1989). To represent RV-E, subjects are to respond to statements like "The country is a better place than the city to raise kids."

Stewardship of Earth ( $R V-S E)$. To the author's knowledge, no one has developed RV-SE as a construct of Rural Values, particularly with a scale to measure it. The notion does appear in literature about rural people, cited at the end of the paragraph, and Stewardship of Earth (RV-SE) has been long accepted as central to rural values. An obligation to care for the land, to protect the land from damage by outsiders, and to preserve the family farm can be found in most rural communities. Rural communities have taken stands against land-greedy encroachments of urban development and "improvements" (such as Super Wal-Mart or Super Big-K). Affected communities have expressed suspicions of government attempts to control areas of land (places like wetlands) as cloaked attempts to wrench the land away from the farmers (to provide hunting grounds for the city-slicker hunter) rather than as attempts truly to protect the 
land. Though not tested as a construct in a study, RV-SE consistently has been found to be a characteristic of rural people by Caldwell (1995), Ching and Creed (1997), Gilchriest (1995), Hemphill (1975), Hofstadter (1963), Little (1998), Peshkin (1994), Reed and Reed (1996), and Wilson and Ferris (1989). In addition, the author's lifelong experience in rural environs suggests that rural populations do indeed express a sense of stewardship for the earth (RFN). In order to capture RV-SE in the questionnaire components such as "Protection of the land is an important value to teach" and "Family farms should be protected" were included.

Good Old Boy (RV-GOB). The final concept evidenced in this study of rural culture is the Good Old Boy (RV-GOB) paradigm. RV-GOB is exhibited as a system that uses key individuals (usually male) to provide frequent preferential treatment and consideration of males in financial, social, or political advancement or in escape from criminal prosecution and prejudicial decisions in civil matters. Although a woman may use the system to advance her cause, the base of power in the system is vested in males (Batson, 1993; Gilchriest, 1995; Hemphill, 1974; Reed \& Reed, 1976; Wilson \& Ferris, 1989).

To the author's knowledge, though GOB seems to be readily accepted as a means to power in rural communities, no instruments have been found that actually assess that element of rural culture and policy making. Therefore, the author was required to rely upon her "intense and prolonged contact" with the community under study (Miles \& Huberman, 1994, p. 6), in order to develop the RV-GOB index. Simply put, the GOB is a network of local men (almost always men) in hierarchical positions who have earned their "GOB stripes." A "stripe" may be earned through hunting, gun collecting, beer 
drinking, drag racing, raising bird dogs, or other "manly" rituals (Gilchriest, 1995). These men take care of the men "under" them. If a problem can't be handled by Man A (at a lower position on the hierarchy), he contacts a man he knows on the next higher level (B), and so on until a man on a high enough level can take care of the problem (see Hemphill, 1974; Wilson \& Ferris, 1989). Preference for male sports programs, leadership positions, career advancement, and local anti-gun control for males are traditions that have been evidenced through the GOB network.

Closely akin to the male-dominant RV-SC/T construct, GOB is self-perpetuating: because there are more males in leadership and decision-making positions, and because there is a male-preference attitude in the traditional rural culture, males continue to dominate the political, as well as the social, scene. Men, and women who know how to use the network, can fill positions even with fewer qualifications, avoid legal punishments, carry on illegal activities without fear of interference from authorities (who usually are part of the GOB system), and get out of trouble by having contacts within the GOB. GOB embraces other RV constructs such as Traditionalism and AntiIntellectualism. In addition, an element relative to Ethnocentricity, a loyalty to country music, is also considered to be a standard of behavior for a Good Old Boy.

Six statements in Section II of the survey deal with Good Old Boy. They express sentiments about respect for manly rituals and male preference. "A pick-up truck, a good hunting gun, and a nice hunting dog are status symbols in our community" and "It's OK for a kid to miss school every once in a while to go hunting with his dad" are two examples. Scoring of Items 
RV items on Section II of the Survey are anchored to a five-point Likert scale. Respondents are instructed to rate how they feel about the statements on a scale of 1 to 5 , with 1 indicating "Strongly Disagree" and 5 indicating "Strongly Agree." The Likert scale is used for several reasons. First, the scale allows for comparison of degrees of agreement in respondents and the five-point scaled responses result in scores that may be quantitatively evaluated (Mason \& Bramble, 1978). Second, the element of time is a consideration in trying to ensure as high a return as possible, and the use of the scale shortens the time needed to respond to each item. Third, a Likert scale eases the task of coding and entering data for analysis. Finally, while a Likert scale is, strictly speaking, an ordinal scale, equating the Likert descriptors to the numbers 1 to 5 on the survey directions and response columns effectively transforms the ordinal scale to interval. Some researchers have commented that this numbered Likert scale can be treated as a ratio scale and is robust to the violation of assumptions when means and standard deviations are calculated on the resulting interval scale (see Crocker \& Algina, 1986; Pedhazur \& Schmelkin, 1991).

Scores for each of the five Parent Values constructs represent the overall mean across the means for each item in the respective scales. Because concept-acceptance is phrased in an inverted fashion for some items, these are reverse scored (see Appendix R). Non-responses are treated as 3 (Neutral), a conservative correction.

\section{Parent Religiosity Constructs}

A number of constructs have been frequently and widely accepted as valid and reliable in establishing comparable scores of the degree of individual Religiosity (Asa, 1995; Bader \& DeMaris, 1996; Cornwall et al., 1986; Darnell \& Sherkat, 1997; Ellison \& 
Sherkat, 1993; Glock \& Stark, 1965; Perrin, 2000; Regnerus, 2000; Sherkat 1997;

Sherkat \& Ellison, 1997). These include Belief (PR-BEL), Experience (PR-EXP), Ritual (PR-RIT), Primary Affiliation (PR-PAFF), and Affiliation Bond (PR-ABOND). One other characteristic, Knowledge (PR-KNOW), was deleted from the original version of the survey instrument because selection of enough items to establish a valid and reliable Parent Religiosity-Knowledge score was not possible given the need to shorten the survey.

Belief (PR-BEL). At the most basic level of PR, Belief is a person's intellectual agreement with a stated or written conviction about a supernatural force or being. Belief neither requires nor reflects action taken to support that conviction. It is merely a spoken "in theory" agreement (Davis \& Robinson, 1996). Section III A of the survey is designed with sixteen statements about belief to which participants agree or disagree, using a fivepoint Likert scale. Three sub-constructs comprise the larger scale. School as Threat is illustrated with five items based on Asa (1995), Bader and DeMaris (1996), Darnell and Sherkat (1997), Gatto (1992), Peshkin (1994), and Sherkat (1998). One example is, "School threatens the values I want my child to have." Another aspect of PR-BEL is Integration of Religion into Schools (Hoffman \& Miller, 1997; Peshkin, 1986 \& 1994; Regnerus, 2000). Beliefs associated with this aspect are reflected in four items such as "We should put the Ten Commandments back in the classrooms." Finally, the concept of a Literalist Belief in the Bible and a Devine God (Darnell \& Sherkat, 1997; Ellison \& Sherkat, 1993) is illustrated by four items like "The Bible is without error or contraction." Ritual (PR-RIT). Ritual (PR-RIT) indicates actions performed by a person in response to her/his beliefs. The more often an action is repeated, the greater the degree of 
Ritual. Section III B is designed to establish PR-RIT with eleven items based on the literature of Cornwall et al. (1986), Davis and Robinson (1996), King and Hunt (1969), Sherkat and Ellison (1997), Stark and Glock (1968), and Wuthnow (1973). Respondents are asked to record how often they perform certain rituals (activities) based on a sevenpoint behavioral scale: from " $1=$ seldom" to " $7=$ more than once a day." A seven-point scale is used to assess the degree of religious activity as precisely as possible. Rituals include attending church, reading the Bible, praying aloud, and watching religious TV. Because the Ritual activity recognized by most scholars as an indicator of religiosity is attendance (Djupe \& Grant, 2001; Dobratz, 2001; Dorahy \& Lewis, 2001; Ellison \& Andersen, 2001; Rowatt, Ottenbreit, Nesselroade, \& Cunningham, 2002; Scheepers, Grotenhuis, \& Van Der Slik, 2002; Sherkat, 2002), three items are appointed to measure attendance at church, in Bible study, and in church related activities. The survey asks about "giving of money to the church because offering of money and/or time for church sponsored projects is identified as another indicator of PR-RIT (Glock \& Stark, 1965; Lam, 2002; Thompson \& Remmes, 2002). Bible reading and studying the scriptures (Orenstein, 2002; Schwadel, 2002) are also considered to be PR-RIT indicative so they are included in Section III B as well. Finally, praying aloud, praying silently, and saying grace before meals (Lam, 2002; Orenstein, 2002; Rowatt et al., 2002; Sherkat, 2002) are thought to be strong indicators of religiosity and are included in the survey.

Experience (PR-EXP). Another concept considered to be strongly indicative of PR is the element of Experience (PR-EXP). According to Glock and Stark (1965), PREXP reflects the degree to which a person has interacted, communed and/or connected with a supernatural force or being (see also Poloma \& Hoelter, 1998). Glock (1973a) 
suggests that RV-EXP can be viewed on a continuum of increasing intensity, thereby creating a true scale. Items describe instances of contact with a supreme entity, increasing in the levels of intimacy. Stark (1999) maintains that in Jewish, Christian, Muslim, and Mormon faiths, believers hold scriptures as communications or revelations "from the actual thoughts of God conveyed to selected recipients" (p. 287), but that the intensity and intimacy of a person's experience with God varies with the believer. The more conservative believers hold that "the chosen people" are guaranteed "prosperity" and the "expiation of sin and fidelity to God are infallible ways of assuring heavenly grace ... in matters of health, family, and employment" (p. 321). Thus, six survey items in Section III A relate occurrences beginning with "knowing God's direction for my life" and culminating with "speaking in tongues" and being "slain in the spirit" as the Pentecostal or charismatic Protestant believer expresses interactive experience with God through "the presence of the Holy Spirit" (p. 322).

Primary Affiliation (PR-PAFF). Data provided by Asa (1995), Bader and DeMaris (1996), and Glock and Stark (1965) suggest the next level of PR, Primary Affiliation (PR-PAFF). In simple terms, this construct holds that a person's Religiosity is reflected by "the company he or she keeps." In other words, if a person professes a high degree of PR, but seeks the company of social and civic groups much more often than the company of church or religious groups, her/his degree of PR is more accurately assessed by a lower measurement of Primary Affiliation. Because the conservative Protestant is concerned about "danger of social contamination" (Sherkat \& Ellison, 1997), he/she would be expected to seek the company of other like-believers. Peshkin (1986) finds that conservative Protestant leadership admonish their followers to avoid fellowship with 
people of "this world." Accordingly, it would be consistent with those sentiments to ascertain the Primary Affiliation of the higher respondent, thereby measuring in part his/her religiosity. In Survey Section IV A, a list of seventeen situations that might prompt a need of a friend is given. Beside the list are three separate columns of numbers, each containing the numbers from 1 ("no chance of choosing from this group") to 5 ("excellent chance of choosing from this group"). The columns are labeled "Church," "School," and "Other (Civic, Social)." Respondents circle a number in each column that indicates how likely they are to choose a friend from each group. This design distinguishes which of the three types of settings provides the most salient primary affiliation for the respondent.

Affiliation Bond (PR-ABOND). According to Asa's (1995) work, the element that most closely identifies a person's true PR is the degree or intensity of the Affiliation Bond (PR-ABOND). How closely connected to a group, how intimately involved, how much in the know, how comfortable in the midst, how missed when absent, how grieved at the thought of leaving, how much is felt "I truly make a difference here," are indications of the PR-ABOND because the bond is tighter when the social ties are greater (Krause, Ellison, Shaw, Marcum, \& Boardman, 2001). The higher the measure of PR$\mathrm{ABOND}$, it appears that the higher the degree of religiosity that will be held by a believer. In Survey Section IV B, PR-ABOND is determined by a participant's selfassessment. For twenty relationships, a subject chooses from numbers on a scale with opposite poles. To prevent bias related to question construction (Tucker, 1994), items are stated so that the extremes of the response scale are equally attractive. Statements are structured around a respondent's role(s) in church, school, social, and civic groups, with 
five statements for each of these settings. Reactions to twenty situations or activities are selected from a scale that numbers 7-6-5-4-3-4-5-6-7. Choices are to reflect feelings about belonging to the group. For instance, "At church I am the" could be rated from "audience" 7 to 3 "neutral" to 7 "participant" at the other end of the scale. "At school I am the" would be rated the same way. The strongest PR-BOND is realized in the role of participant rather than audience, of insider rather than outsider, of close friend rather than acquaintance, or of needed rather than unneeded.

Scoring of Items

Section III $\mathrm{A}$ is anchored to a five-point Likert scale, with answers ranging from 1 (Strongly Disagree) to 5 (Strongly Agree). From these responses, the mean and standard deviation for items that concern PR-BEL and for items that concern PR-EXP can be determined. The two scales represent average scores across their respective individual items. Non-answers are coded 3 (Neutral).

Section III B provides a PR-RIT score by figuring the mean for all answers selected as responses to the 11 statements and then averaging across these scores. Nonanswers are coded as the neutral score for that item.

Answers to items that concern PR-PAFF on Section IV are selected from a range of numbers on each of three columns, from 1 (no chance of choosing from this group) to 5 (excellent chance of choosing from this group). Church, School, and Civic/Social Groups represent three distinct measures of PR-PAFF; scale scores are the average across the eleven statements for the three respective settings. This method of response provides an indicator of the likelihood of Primary Affiliation, from negative (low numbers) to positive (high numbers). Those numbers suggest the respondent's Primary Affiliation 
and Primary Non-Affiliation. The mean PR-PAFF Church score is utilized as part of the PR block for this study. The PR-PAFF School and Civic/Social scores represent supplemental information to be used for comparison. Missing values are coded as 3 (Neutral).

The final PR score, PR-ABOND, was gathered from responses to statements of "belonging" or "participation" that are indicative of bonding. Numerical responses are converted to points on a nine-point scale. Beginning with the seven on the left, numbers are assigned points in increments of one: $7=1,6=2,5=3,4=4,3=5,4=6,5=7,6$ $=8,7=9$. Missing values are coded as 3 (Neutral, five points). Scores for degree of bonding to church, school, social, and civic groups, as sensed by a participant, are obtained from the mean across responses to the five statements pertinent to each group. (See Appendixes $\mathrm{Q}$ and $\mathrm{R}$ for specific questions to the four groups.)

\section{Mediating Factor}

As has been discussed, the process of answering the Research Questions includes identification and measurement of Independent Variables, including Demographics and Parent Values, and assessment of their relationship to the Dependent Variable, Student Outcomes. However, a third type of Independent Variable, Parent Involvement, may affect those relationships, directly or indirectly. Hence, identification and measurement of PI (and the two PI constructs) as a Mediating Factor in the interrelationships, is necessary.

\section{Parent Involvement Constructs}

Parent Involvement (PI) is identified in this study as the third type of Independent Variable, the Mediating Factor. It is examined for its relationship to Student Outcomes 
(SO), i.e., as it mediates the relationship between Parent Values (PV) and Student Outcomes (SO) while controlling for Demographics (D). Constructs that constitute the foundation for Parental Involvement include Belief (PI-BEL) and Activity (PI-ACT).

Parent Involvement-Belief (PI-BEL). Research by Epstein (1995), O'Neil (1991), and Steinberg (1996) indicates that PI must be valued by parents and schools in order for significant increases in SO to occur. Parents may be involved in their children's education in many ways, but the extent of their involvement usually relates to what they say they believe about the value of Parent Involvement and to what they think the school community believes about the value of PI. For this study, those stated beliefs are construed as the Parent Involvement-Belief (PI-BEL) construct.

Section I A of the survey is designed to determine a parent's belief about Parent Involvement (PI-BEL). Subjects are asked to react to five statements of belief concerning the value of PI by choosing numbers (anchored to a five-point Likert scale) from 1 (Strongly Disagree) to 5 (Strongly Agree). The items cover statements about parent beliefs, child beliefs, and teacher beliefs. For example, one statement is, "My child's teacher(s) consider(s) me important in my child's education."

Parent Involvement-Activity (PI-ACT). The "walk of the talk" is probably more reflective of PI-BEL than statements of belief. The actual deeds parents perform or moves they make to support their child's education are probably more truly indicative of their real philosophy about PI. Such actions are referred to as the Parent InvolvementAction (PI-ACT) construct. PI-ACT includes activities performed at home or outside the school domain or at school or within the school domain. Some experts, including Epstein (1995), consider home activities and support as passive involvement, and they claim that 
active involvement, school domain activities, is more valuable because it is more visible and more easily identified and appreciated as PI by the child.

Section I B consists of fourteen statements designed to assess the type of PI actions that parents perform and the degree or duration of such parent activity. Epstein (1995), O'Neil (1991), and Steinberg (1996) find that PI may take many forms, both at school and in the home, and that the most effective PI is active involvement (PI-ACT)-parents actually doing something rather than being a passive audience member. They find that parents must be involved as often, as much, and as long as possible for children to gain as much as possible from their educational opportunities.

To assess the number of activities and the duration of those activities parents profess, the survey requests respondents to indicate how often they perform activities by circling the letter that "MOST CLOSELY reflects how often" they do the activities. Letters provided as answers are $(\mathrm{N})$ Never, (S) Seldom, (O) Often, (F) Frequently, and (A) Always. Statements of activity include items such as, "I attend Parent Teacher Organization meetings," and "I provide a study area with needed supplies at home." Section I C (Parent Involvement Organizational Activities and Leadership Roles) consists of one open-response question: "In what ways are you involved in your child's education, either at home or at school? Name all that you can think of. Include school groups you are involved in and leadership positions you hold in those groups." Only four blank lines are provided for the answering of the questions because providing more space for open-response questions has been shown to produce only marginally more words and no greater number of ideas (Gendall et al., 1996). Responses are used as supplemental data and/or utilized in designating level of involvement for selecting interview subjects. 
They are not utilized directly for answering the Research Questions.

Section V of the student survey also asks for a record of all activities in which the students are involved. Checklists are provided for school activities (apprenticeship, band, cheerleading, etc.) and other organized activities (motor cross, missions, dance, etc.). Blanks are provided for "other" activities. Blanks are also supplied for listing leadership roles that are filled by the students (president, team leader, etc.). These extracurricular activities are deemed indicative of PI because, without parent support and PI, the students would not have the capacity to be involved.

Scoring of Items

A score for the PI-BEL construct is determined by averaging mean scores for each item on the Likert scale on Section I A. Missing values are treated as 3 (Neutral).

For the PI-ACT score, the letters representing behavioral frequencies in Section I $\mathrm{B}$ are converted to numbers: $\mathrm{N}=1, \mathrm{~S}=2, \mathrm{O}=3, \mathrm{~F}=4, \mathrm{~A}=5$. Missing values are coded as 3. The scale is derived from the average across the mean of each item and recorded as PI-ACT score.

\section{Dependent Variable}

The stated purpose of this study is to gather information that might, ultimately, lead to the development of a plan that could increase Parent Involvement, and, therefore, Student Outcomes. Through an understanding of interrelationships between Demographics (D), Parent Rural Values (RV), Parent Religiosity (PR), and Parent Involvement (PI), educators might possibly devise a plan for manipulation of the parent elements, and, consequently, improve student achievement. Thus, the Dependent Variable is Student Outcomes. 


\section{Student Outcomes}

SO can be measured in several ways. Statewide achievement tests given at the end of each year were considered as potential data for the quantitative portion of this study. However, investigation of those measures revealed several difficulties.

Over the course of grades seven through eight, a transition from one test to another caused students to take portions of two basic achievement tests: Kentucky Instructional Results Information System (KIRIS) and the Kentucky Core Content Test (KCCT) of the Commonwealth Accountability Test System (CATS). This transition resulted in a three-year span for the collection of data: to obtain all of the grade 8 cohort scores, collection had to go back one year; to obtain all of the grade 7 cohort scores, collection had to go forward one year. Seventh graders were assessed in math and social studies in 1998. In 1999, the seventh graders took tests in English, math, science, and social studies. The next biennium, seventh graders took tests in reading and science and were evaluated on their Writing Portfolios and On Demand Writing in 2000; in 2001, the assessment for seventh graders was in math and social studies. Eighth graders, on the other hand, covered the required assessments during two other biennium. In 1997, they were assessed in math and social studies. In 1998, the assessment was English, math, science, and social studies. Reading, science, Writing Portfolio, and On Demand Writing comprised their assessment for 1999, and math and social studies made up their 2000 assessment. Furthermore, the KIRIS and the test that replaced it, KCCT, are Kentucky teacher-designed instruments--they are not nationally normed. In addition, the KIRIS changed in content during the years in question.

Due to all these factors, there are gaps in test coverage for every test and gaps in 
test scores for many students and there is inconsistency in test data and scores. Thus, the KIRIS and KCCT are not comparable. Moreover, a Comprehensive Test of Basic Skills or Stanford Diagnostic test was not given to this group of students. On the other hand, these inconsistencies are far more problematic for the state's accountability system than for the current study. For determining the relationship between Parent Values, Parent Involvement, and Student Outcomes, the particular measure used is less important than comparability of content for the students in the case study, both seventh and eighth graders.

Hence, taking all of these factors into account (different accountability systems, different content tests, both seventh and eighth graders, and the study timetable), the only feasible measure of Student Outcomes is student cumulative Grade Point Average (GPA).

\section{GPA as Student Outcome}

Given the problems for statewide accountability assessments, the most consistent measurement for the two grade levels under study is the GPA. At the end of each school year, the students' final grade averages in four "core content" classes are recorded on permanent record folders. Those core content areas are social studies, science, math, and language arts. Grades are recorded in numerical form on a scale of 1-100 (percentages). A student's overall GPAs across the four core content classes over the two-year period under study (1999-2001) are secured and the mean is recorded.

\section{Supplemental Qualitative Data}

While it is well understood that a single researcher should use "a variety of methods to gather information, rather than relying on a single source" (Ray, 1954, p. 64) because triangulation of data increases a researcher's ability to counter bias, the purpose 
of qualitative research has been to discover contextual understanding rather than to generalize (Maykut \& Morehouse, 1994). Qualitative research examines issues in descriptive narratives as they represent a participant's experience in a situation, focusing on the meaning that the events and elements of the situation have on the participant. The researcher works to find meaning for those involved in the study through interpreting participants' words, actions, and records through patterns that emerge from the data.

Of greater note, however, it has been said (Thorkildsen \& Stein, 1998), "Many researchers over interpret their findings. In many cases, undue importance is given to findings of statistical significance" (p. 17). Emerson (1983) further contends that a formal investigator using mere statistical analysis will be denied meanings and insights that may be had through identity, sympathy, and close familiarity with the subjects. For, while "statistical significance provides some information about the probability of chance occurrence with particular sample sizes," it "does not provide information about the importance of results" (Thorkildsen \& Stein, p. 17). If the society under study is assessed according to statistical findings that have little or no importance to that culture, then it may be said that the data are insufficient, possibly even misleading. According to Emerson, when practitioners want to understand the meanings of observable activities for those involved, immersion in that world is critical for empathic understanding to occur. Because as Maykut and Morehouse (1994) suggest, some knowledge is tacit (we know it but we cannot articulate it), approaches to gathering information must be gained by means in addition to "counting" so that we can experience rather than merely attend to the pieces of our puzzle.

Emerson (1983), in his discussion of "etic" and "emic" accounts, illustrates the 
necessity of providing both perspectives to achieve the most accurate depiction of a culture: etic descriptions use concepts from outside the culture studied and contrasted with emic descriptions that use concepts coming from within the culture studied. For the development of a "thick description," events and scenes relevant to those involved are presented in close detail to capture the context and interconnectedness, "tied together in textured and holistic accounts of social life" (p. 25). "Thick descriptions" allow experience-distant theorists to capture the essence of social life so that generalizations might be possible, justifying the pursuit of "meaning-rich, context-sensitive, and holistic descriptions" for "subtle interpretation and communication of symbolic meanings" (p. 28). Specifically, qualitative approaches, contribute insight "into the relationship between theory and practice" (Ray, 1954, p. 61).

The recognized need for triangulation of data in a study, the possibility of misleading statistical data, and the need for emic as well as etic data in a cultural case study justify the use of supplemental qualitative data. Several sources of supplemental qualitative data are recommended (Bell, 1993): interviews, document analysis, and Researcher Field Notes.

\section{Interview Schedules}

Interview questions prepared for various informants include questions designed to elicit responses that shed light on numerical findings that pertain to the constructs under study. Those questions were created following a pilot of the survey that provided general areas of concern through content analysis of returned questionnaires. The number of questions included in the interview schedules was kept to a minimum, ranging from six on the Withdrawn Student Interview schedule to fourteen for the Student Interview 
schedule, because a "tighter design" is recommended for studies with well-delineated constructs (Miles \& Huberman, 1994). Primary questions are accompanied by a Reviewer's Checklist that provides a series of back-up questions intended to probe hesitant responders. All interview schedules and checklists are presented in Appendix T.

\section{Interview Analysis}

A variable-oriented analysis was employed for reporting interview data because the approach seems more pragmatic in a study of established constructs. Codes that reflect the constructs under study were added to the margins of transcripts to indicate information specific to the designated area of concern. Next, a "gathering" of those comments was conducted and presented in a variable-oriented text. Qualitative Analysis is thereby used to supplement and triangulate quantitative findings. According to Miles and Huberman (1994), exploratory studies can be greatly enhanced through the used of interviews that add interest, depth, and triangulation, and Bauman and Adair (1992) find that ethnographic interviews help identify factors and unanticipated areas of importance.

\section{Document Analysis}

According to Babbie (1989), content analysis is an unobtrusive research method that can supplement other data (Yin, 1989). Content analysis used in this case study is document analysis, whereby documents are studied and catalogued using a systematized protocol so that data within the documents about the constructs can be analyzed. Information gleaned through other research methods may be supported by consistent data discovered in the content analysis. On the other hand, anomalous data may provide rationale for additional study. Several types of documents are used in this exploratory case study: the DEMS School Report Card (Dixon, 2000) (Appendix B), electronic mail 
forwards (Appendixes E, F, G, H, V, W, X, Y, Z, AA, and BB), a local newspaper, and Parent Sign-in Sheet (Appendix A).

The School Report Card supplies data for construction of the DEMS environment. Those data also establish a school baseline against which individual student outcomes, an important construct in this study, may be viewed.

Electronic mail forwards are included to provide unsolicited documentation of local cultural perceptions and values. They are analyzed for ideas and comments associated with the constructs recognized in this exploratory case study.

Transcripts for students enrolled in the study are analyzed for Student Outcomes. GPAs were obtained from permanent student records. Those scores are also components of the interview selection matrix, helping to identify high-, middle-, and low-achieving students.

Parent Sign-in Sheets are also used in the selection process for interview subjects. They help to identify parents who are highly-, moderately-, and slightly-active in their child's education. That information helps to generate the interview selection matrix.

\section{Researcher Field Notes}

A social scientist constructs a social reality by selecting and interpreting elements that exist within the culture under study. A participant-observer, who comes from the "inside," reverses that action of a typical ethnographic social scientist. Instead of constructing a social reality, the insider steps "outside" to view the setting while relying on the "vast background of shared meanings" and the "deeper form of experiential learning" (Emerson, 1983, p. 15) available to him/her. The "researcher's personal records of insights, beginning understandings, working hunches, recurring words or 
phrases, ideas, questions, thoughts, concerns and decisions made during the research process" (Maykut \& Morehouse, 1994, p. 68) are invaluable. According to Denzin (1978), these Researcher Field Notes (RFN) allow for participant observation simultaneously combined with document analysis, interviewing, and introspection.

Strong, Silver, and Robinson (1995) used work by Schlecty (1994) for a study of student engagement. They found that engaged students exhibit three characteristics: attraction to work, persistence in their work, and visible delight in accomplishing their work. These characteristics cannot be assessed through achievement tests, GPA, interviews, document analysis, or quantitative analysis of surveys; they must be witnessed and understood by another individual. For this reason, immersion or indwelling is an essential characteristic of action research (Bell, 1993; Cwick, Wooldridge, \& Hogan, 2001; Maykut \& Morehouse, 1994), which establishes methods of capturing emerging patterns of data because only a human instrument can be "flexible enough to capture the complexity, subtlety, and constantly changing situation which is the human experience" (Lincoln \& Guba, 1985, p. 26). RFN are "ongoing descriptive reports ... [and] the means by which the inquirer makes the implicit (the subsidiary) explicit (articulate)" (p. 33).

RFN collected over the time span of this study provide documentation of student, parent, teacher, administrator, and community behaviors as well as elements observed in the environment that comment on the study constructs (for example, the number of pages of the local paper dedicated to sports as opposed to the number dedicated to academics). Marginal notations commenting on mood, tone, atmosphere, and/or extenuating circumstances amend behavior observation notes. Through common experiences, 
through exchanges of social realities, and through shared motives, intentions, and emotions, the researcher supplies the "thick description" and context for the study through the RFN.

\section{Validation Process}

Items have been included in the surveys and interviews after peer review, after a pilot study, and after scrutiny, critique, and acceptance by experts in the fields. Types of validity and issues related to participant-observer status are also addressed in this section.

\section{Peer Review}

Most of the survey questions were developed while the researcher was a student in a doctoral seminar. The questions were submitted for critique to a panel of peers who were also in that seminar. Format and length of the survey were revised as a result of the critique.

\section{Pilot Study}

The Parent Survey was administered to a college English 102 (Writing Research) class. It was presented as an educational activity in preparing surveys for the students' own research projects. Students were asked to review the instrument, making note of any confusing directions, grammatical or mechanical mistakes, difficulties in answering a particular item, offensiveness of any item, or items causing anxiety. They were also asked to complete the survey, assuming the role of a parent of a student at DEMS. Completion of the pilot study took approximately fifteen to twenty-five minutes.

Following the completion of the surveys, the results were reviewed and comments concerning the instrument construction were noted. The survey was then revised to address the concerns of individuals taking the survey. Particularly, Section IV A, which 
asked subjects to choose a number from each of three groups for seventeen different situations, was revised to clarify and emphasize unclear instructions.

Results of the survey suggested that the items do indeed reveal patterns and trends toward conservative rural and religious values. Parental involvement scores were low as expected, considering the fact that the participants were role-playing.

\section{Experts in the Field}

The author's Dissertation Chair, Dr. Stephen Miller, University of Louisville, and Dr. Mary O'Phelan, Western Kentucky University, read and scrutinized the study. They both have extensive experience with methodological issues and survey construction. They recommended changes which included re-wording to reduce bias, consolidating of information to cut length, and screening for objectivity in the quantitative section.

Further changes to the survey resulted from a review of some of the items by other experts in the field. For example, Dr. Darren Sherkat, who is also a member of the researcher's committee, is a faculty member in the Department of Sociology at Southern Illinois University, Carbondale (previously at Vanderbilt University). He is, furthermore, an often-published expert in the field of Sociology of Religion. He suggested additional items for inclusion in the survey to strengthen validity of the Religiosity Constructs. $\mathrm{He}$ also recommended the elimination of the PR-KNOW construct from the survey because of lack of space to assess that element adequately. Dr. Christopher Wagner and Dr. Dwight Cline, also members of the members dissertation committee, provided insight into Parent Involvement and Rural Values respectively.

Dr. Paula Little (1998) also reviewed the study. In the process of completing her dissertation, Dr. Little prepared a benchmark survey in the area of Rural Values. In her 
critique of this study, she suggested methods of strengthening the validity of the Rural Values section of the survey and eliminating redundant information.

\section{Validity}

There are several areas of validity that need to be addressed. Within the preinstrumentation domain, those areas include construct validity and descriptive/contextual validity. Within the instrumentation domain, they include response bias, face validity, and internal/content validity.

\section{Pre-Instrumentation}

It is important that, before a study is conducted, researchers ground their exploration in a broad range of information provided by previous researchers in the field of exploration. A study needs to be built on the foundation laid by prior research. That foundation lends validity to the constructs of any new study.

Construct validity. When a study is used to make inferences about an audience and the characteristics it exhibits that are consistent with existing theory, it is said to have construct validity (Noll, Scannell, \& Craig, 1979). Numerous studies have been examined and referenced in this exploration that substantiate construct validity for the study. The concepts are well grounded in previous research, as is required by research scholars (Miles \& Huberman, 1994).

The Parent Religiosity Constructs have all been previously identified and assessed over a long period of time by many experts in the field, such as Asa (1995), Ellison and Sherkat (1993), Glock and Stark (1965), and Wuthnow (1973). Most of the Rural Values Constructs have been identified and studied by many experts in this field as well. Those experts include Ching and Creed (1997), Hofstadter (1963), Howley and Howley (1995), 
Little (1998), and Peshkin (1994). Epstein (1995), O’Neil (1991), and Steinberg (1996) are some of the leading experts in the field of Parent Involvement who have previously identified and assessed those constructs. Noll, Scannell, and Craig (1979) provide the theory upon which some survey items were based. In addition, specific to this study is the work on Rural Values proffered by Ching and Creed (1997), DeYoung and Laurence (1995), Fox (1997), Hofstadter (1963), Porter (1973), Thurber (1997), Verhoog (1995), Verkleij (1995), and Witte (1995).

Descriptive/Contextual validity. The descriptions of Dixon and the middle school have been designed to establish descriptive/contextual validity vis-à-vis experts in all of the areas of concern: education in the rural South (Hosftadter, 1963), fundamental conservatism in the rural South (Peshkin, 1986), Rural Values (Little, 1998), affiliation (Asa, 1995), Religiosity in the rural South (Sherkat \& Darnell, 1999), Parent Involvement (Epstein, 1987), and middle schools (Barber \& Patin, 1997). Connection to previous research settings and details assists in providing congruity, and therefore, descriptive/contextual cogence.

\section{Instrumentation}

Only if a study is valid does it have any use. It must appear to do what it is designed to do (Noll et al., 1979), and it must be put through trial runs in order to establish its probability for accuracy. Triangulation of the data obtained during the study also serves to gain credibility or validity.

Response bias. Section III B on Parent Religiosity-Ritual (PR-RIT) may be the most vulnerable to response bias. This self-report of frequency of behaviors may tempt the respondents to give themselves a higher score than is appropriate. Two methods of 
checking for this bias are applied. First, the student and the parent are completing the same items. A request was made in the cover letter for the parent and the student to take the survey without revealing answers to the other party. Those answers are compared to triangulate the data requested. Second, the Religiosity score for the parent depends on factors in addition to the Ritual scale. Because the Belief and Experience scales do not readily promote response bias, it was anticipated that a combined Religiosity score establishes stronger validity.

In spite of the possibility of response bias, the information gleaned from Section III B concerning PR-RIT as it correlates to SO and PI may be found significant. Economic and education factors (SES) and Family Structure (FS) have been shown to correlate to parent choice of worship, as well as to PI and SO. Religions considered most conservative have been shown to attract people of the lower SES levels. Parents in the lower SES levels have been shown to reveal lower PI and SO scores. Since these relationships have been demonstrated in previous research (e.g., Epstein, 1995), a similar finding in the current study would strengthen the finding of construct validity for this variable (see above section on Construct validity).

The check against response bias by comparing student responses to parent responses can be utilized in a number of different variables in the study, in addition to PR-RIT, because the parent and student surveys were designed in parallel for several of the constructs in Figure 1. This cross checking on different response patterns within the same family provides not only a prima facie comparison but also utilizes the fact that the students may be less "sophisticated" about how their answers "appear" and therefore less likely to reflect social desirability "corrections," whether conscious or unconscious. 
Face validity. According to Borg and Gall (1989), face validity "refers to the evaluator's appraisal of what the content of the test measures" (p. 250). The descriptive portions of this study mirror much of the work done by Peshkin (1986) in his study of a fundamentalist Christian school and in his 1994 study of a rural school. That fact constitutes an element of face validity for the study. Because many of the questions on the surveys rely heavily on questionnaires prepared by previous researchers, face validity is again supported.

Instructions provided in the survey inform the respondent that the survey is designed to determine how the parents feel about how well their school responds to parents. Most pilot respondents confirmed the face validity of the survey in this respect.

Internal/Content validity. A survey must measure appropriately what it is intended to measure (Noll et al., 1979). In order to assess the content validity of the instrument, the survey was administered to different groups prior to its final revisions. As noted above, numerous peers of the author and students in the author's writing classes completed the survey. Most significantly a pilot study was conducted using students in the author's research class. Data from these "pre-tests" were gathered and analyzed. The answers proved to be somewhat predictable and no extreme pattern of outliers was obtained. The survey items seemed to test what they were intended to (Borg \& Gall, 1989).

Miles and Huberman (1994) assert that validity is improved with mixed-design. This study accomplishes the elements of qualitative design with a thorough description of the setting, of the population, and of "what exists" within other elements of this culture. The author relies extensively on Researcher Field Notes taken during her twenty-year 
tenure in the school system. Interviews with parents and students provide supplemental data to further personalize the details. For a quantitative approach, the author utilizes surveys she constructed for parents and students. Data gathered from those surveys were analyzed in accordance with the research questions (Table 2). Thus, the study's mixed design and various methods of obtaining data strengthen overall validity. For example, triangulation of the data collected from parent surveys were secured in several ways: through student surveys; through interviews with parents, students, others; and through perusal of Researcher Field Notes. The triangulation offers further grounds for internal validity.

\section{Participant-Observer}

Miles and Huberman (1994) claim, "In qualitative research, issues of instrument validity and reliability ride largely on the skills of the researcher" (p. 38). To that end they suggest several markers of a good researcher-as-instrument: familiarity with the setting and the phenomenon, strong conceptual interest, and good investigator skills that help the researcher "dig deeper" or probe further during interviews. There is no doubt that this researcher has familiarity with the setting and the content as well as strong interest in the study.

Field notes of researcher observation during the preceding years as a teacher at Dixon and a member of the community and culture under study provide one source of data for this exploratory case study. The researcher has been a teacher at DEMS for twenty-one years, a resident of the Dixon area for over forty-five years, and a member of the Dixon First Baptist Church for more than ten years. However, in addition to her insider status, the author is atypical of the Dixon rural resident in substantive ways that 
provide a great degree of academic objectivity and distance. The researcher brings a strong academic background to the study. She has continued to achieve in the field of education during her teaching tenure: she has been trained and recognized as one of Kentucky's first Distinguished Educators; as a KERA Fellow; as a member of the first state Writing Assessment Committee at the beginning of KERA implementation; as a Regional Service Center Associate; as a Purchase Area Writing Project Fellow (PAWP); as co-director of the PAWP; as co-director of the advanced PAWP project; as a teacher of English 101 and 102 classes for Madisonville Community College, Henderson Community College, and Murray State University; as a published author; and as a lay missionary/teacher to Russia. She has served as School Transformation Plan (STP) Consultant for the state of Kentucky; as Writing Consultant for many school districts; as Professional Development Leader for many teacher groups; as President of the Kentucky Council of Teachers of English/Language Arts; and as member of the National English Standards committee. She has memberships in many educational and religious study societies: Association for the Scientific Study of Religion, National Association for Social Studies, National Council of Teachers of English, National Middle School Association, Phi Delta Kappa, National Rural Educators Association, National Association of Secondary School Principals, and Association for Supervision and Curriculum Development. She has presented programs at state and national conferences. She has served on the review boards of two textbook companies and on the editorial board of a children's magazine, and she was the co-director of Supporting Teachers as Researchers (STAR), an advanced writing project program at Murray State University. She is well known (in outside academic circles and in religious literature circles) for her 
researcher-participant studies in her own classroom and for her writing skills, both in academic circles and in religious literature circles.

\section{Participant-Observer Bias}

Though the researcher-participant role leaves much room for criticism concerning researcher bias, several factors help to outweigh the chance for bias in favor of the participatory role. The xenophobic tendencies in conservative, rural communities like Dixon preclude the involvement of an outsider (Cline, 1997). Despite the objective benefits inherent in being an outsider, the benefits of being an insider are much greater. As Little (1998) explains in her intensive and thorough study of rural values and organizational fit, rural people have ethnocentric attitudes or tendencies--"the tendency to view their own race, religion, culture, or group as superior to others" (p. 85). Also, Little cites primary group preference as another rural value that would handicap an outsider in an honest assessment of the setting and culture of this rural community. Primary group preference leads individuals to have a few close family members and friends. Major decisions and discussions are most often conducted only in the presence of those close group members, and outsiders find gaining trust and openness a difficult task. Therefore, cultural sensitivity is almost a prerequisite for a study such as this one. Qualitative, directly gained, research data collected by an outsider could be criticized in areas of reliability and validity, but the author provides the cultural sensitivity needed.

The researcher is an insider and, as such, has access to the inner circles of many primary groups, and the perception of risk in answering her survey would not be as great. She is not viewed as a threat because she has lived most of her life (excluding time spent in undergraduate programs at college) in the Dixon area. Her involvement in the Dixon 
FBC provides her insulation from the distrust of "anti-religious, egg-head, educators from the city." Her lifetime connection to her father and her father's farm and farm hands give her an inside seat to the theatre of the family farm and southern rural life.

While maintaining close ties and identity with the Dixon community (fifthgeneration resident and land owner, Music Leader in her church, leader in local civic, social, and church groups) the researcher "guards" the incognito academic identity that provides her status in academic circles. In order to prevent generating distrust and ostracism, few select individuals are aware of the researcher's advanced academic accomplishments.

Often researchers must exert much energy and time to be welcomed and trusted by the population in a case study (Peshkin, 1994). This researcher has spent more than forty years in the field, interacting, observing, learning, and securing acceptance as a conservative, rural resident of the Dixon community. Because she is widely accepted and trusted as "one of their own," she is privy to parent and student opinion that is honest rather than modified, forthright rather than censored. An outsider would be captive to the designation of "outsider" and his/her qualitative data would be heavily affected.

Therefore, though she does not provide outsider objectivity, the author does provide many other positives. As a former teacher (recently retired) at DEMS, the researcher is a participant-observer in this case study. Though that role presents cause for concern about researcher bias, the role does provide the researcher with a unique familiarity and understanding of the community under study. She holds simultaneously the status of a rural insider, an ecclesiastical insider, and a dedicated lifetime academician. 
Because she is concerned about her rural community and its academic status, the researcher attempted to conduct objective research so that valid data could be collected. She focused on the data in order to glean a fuller understanding of the overlapping, interwoven aspects of PR, RV, and PI. For that reason, it was to her advantage to work diligently to protect the validity of the data.

\section{Analysis of Data}

Because descriptive studies seek to establish "what is" (Borg \& Gall, 1989), descriptive statistics are reported to establish the qualities of the community under study for all of the variables listed. Psychometric analysis of scale reliabilities will utilizes Cronbach's (1951) coefficient alpha. To determine the degree of relationship between variables, a correlation matrix is computed. Subsequent analyses such as multiple regression describe the strength of relationship between several Independent Variables and the Dependent Variable, consistent with the research questions. Because of the exploratory nature (not predictive) of this study and the attempt to examine relationships, a significance level of .1 was established. (The small sample size in the case study also contributes to this decision.) The adjusted $R^{2}$ represents the effect size in the regression analysis. A correlation matrix constitutes the underlying basis of all subsequent regression analysis for the research questions. Table 2 provides a summary of the specific methodological procedures associated with each different question. As needed, scatterplots or scattergrams are utilized to represent the possible relationships between variables.

The current case study has a limited number of individuals $(N=109)$ and the survey of parents/students netted only 38 . This low working number of cases represents 
a severe limitation in data analysis. Because the theoretical model (see Figure 1) has several constructs, including three conceptually distinct types of independent variables, the ratio of number of cases to number of predictor variables in the regression analyses is suspect. Ideally Stevens (2002) suggests a ratio of $15: 1$. Even with a $100 \%$ return, the analyses in question fall short of Stevens' recommendation. Although Allison (1999) states that a ratio of 5:1 may be adequate, clearly this issue is problematic in the current study.

This problem of low case numbers is dealt with in two ways. First, the type of regression utilized for Research Questions 1-6 is stepwise, which is appropriate in exploratory research to establish which predictor variables actually have a significant influence on the outcome variables (see Tabacknic \& Fidell, 1996). Stepwise regression also helps ameliorate the problem of low ratio of cases to predictor variables by ordering the independent variables by the strength of their association with the dependent variables, from most to least, with only the significant variables retained in the equation. Second, the analyses called for in the research questions (see Table 2) will be done in subsections. Because the number of cases may not permit testing the full model (Figure 2) in a single computation, subgroupings of variables are examined for their effect on Student Outcomes. Non-significant variables from these analyses are eliminated from subsequent equations as the different sub-groupings are combined. While this procedure represents a stopgap approach, the result should permit identification of the key significant independent variables in the model, with a limited ability to examine the combined effect of these key constructs in subsequent analysis across the subgroupings. 
This subgroup approach should work reasonably well for Research Questions 1-6, which represent direct relationships (Table 2). The more problematic analyses are Research Questions 7-10 which represent the combined indirect and direct relationships, particularly the full model posited in Research Question 10. For these research questions, hierarchical regression is required; while only significant variables from Research Questions 1-6 are retained for these computations, the number of different blocks involved pushes the limit on even the 5:1 ratio.

The limitations on the quantitative analyses just described must be seen in the context of the purpose of the study. The case study is explicitly exploratory, and the goal is theory development, not model testing. The hypothesized relationships developed in Figure 2 represent a synthesis of the literature in several fields, particularly rural values, parent religiosity, and parent involvement, all in the context of a southern rural culture in a small middle school. The approach outlined here permits examination of the model, albeit in limited fashion. However, the supplemental qualitative analyses provide further corroboration for any conclusions that may be made. On balance, the study should still provide useful data on the validity of the proposed theoretical model. That said, further investigation with larger more representative data bases across a number of schools is required to replicate any findings from this study. But that statement would also be true even if this school had an adequate number of individuals to satisfy requirements for multiple regression. Apart from the number of parents/students within the school, the research examines a single school, and the strength of any case study is its internal validity, not its external generalizability.

Human Subjects Review Board 
Religiosity occupies a position in the sensitive research area. The ethics of sensitive research include guarding against alteration of the subjects under study, against endangerment (or sense of endangerment) to the subjects or their belief systems, and against subject bias in response to perceptions of "outsider" judgment.

In response to these concerns, the researcher registered for the Human Subjects Review training required by the University of Louisville. After completing the training, the researcher passed the test designed to ensure a researcher's understanding of human subjects' rights under current laws and regulations that protect human subjects; this resulted in certification.

Next, the researcher completed the University of Louisville Human Subjects Review Board (HSRB) application with a request for expedited approval process. She was asked to supply a separate and more specific agreement (consent) form for participants to sign, and, after that form was submitted, the University of Louisville approved the study.

Because the study was conducted in partial fulfillment of the requirements for the degree of Doctor of Philosophy in a cooperative program between the University of Louisville (UofL) and Western Kentucky University, the researcher was required also to apply to the HSRB at Western Kentucky University (WKU). In order to obtain approval from WKU, the researcher prepared and submitted a separate signature form for the planned interview process. Upon receipt of that additional form, WKU HSRB approved the study (Appendix CC). At the end of one year, and at the end of two years, reapproval for the study was requested and granted by UofL and WKU (Appendix DD).

Summary 
The purpose of this study is to examine the interrelationships of Conservative Christian Parent Religiosity (PR) and parental Rural Values (RV), as mediated by Parental Involvement (PI) and controlling for Demographics (D), to Student Outcomes (SO) in a southern rural middle school. It is an exploratory case study of mixed design that uses quantitative and qualitative methodology: surveys, interviews, student accountability data, document analysis, and researcher field notes. Subjects for this study are the 109 seventh and eighth grade students at DEMS and their parents (during 19992001). Sources include those parents and students and support personnel and faculty of those students in addition to student achievement data. Surveys of parents and students were self-administered by mail; surveys included a cover letter, instructions, selfaddressed-stamped envelopes, and a token payment of a dollar bill to enhance return rate. There were ten Research Questions to be answered, based on an exhaustive literature review, with Independent Variables of Demographics, Parent Values, and a Mediating Factor, examined for their influence on Student outcomes, as conceptualized in Figure 1. Provisions for construct, descriptive/context, interpretive, face, and internal/content validity have been applied. Quantitative computations include descriptive statistics, psychometric analyses, and multiple regression. Qualitative analysis provides a supplemental perspective for triangulation. Human Subjects Review Boards from the University of Louisville and Western Kentucky University have approved the study. 


\section{CHAPTER IV}

\section{RESULTS}

Many researchers have investigated parent involvement and its correlations to student outcomes. Few, however, have studied the overlapping impact of parent rural values, parent religiosity, and parent involvement on student outcomes. To that end, this is a case study of 109 students from a small rural, middle school in Western Kentucky and their parents, staff, and community. It is an exploratory study of the interrelationships of rural values, parent religiosity, parent involvement, and student outcomes within a southern, rural county that is predominantly white and Protestant Christian.

The primary data collection was a survey, designed in parallel form for parents and students, that covered three distinct types of Independent Variables, consistent with Figure 1, the Theoretical Model (p.12). The survey netted 38 from 109 parent/student pair replies after one mail reminder, three electronic mail reminders, and several random telephone reminders. The survey utilized Likert and Likert-type scales; operational definitions for all scales and variables are attached at Appendixes Q and S. Supplemental data were gathered from interviews with nine parent/student pairs, two support staff at the school, and one faculty member. Student Outcomes are Grade Point Averages recorded as percentages, collected from the office of the high school guidance counselor. Results of this study are presented in the remainder of the chapter under four sections: Descriptive Statistics, Psychometric Analysis of Scales, Research Questions, and 
Qualitative Analysis.

\section{Descriptive Statistics}

The descriptive statistics presented below are ordered by the variable categories in the theoretical model (Figure 1). The 109 survey requests sent out to parents of the seventh and eighth grade students from Dixon Elementary Middle School (DEMS) merited an "effective return" $N$ of 38 . For the Demographic Controls, data came from parents' responses to the demographic sections of the survey. These factors represent the first of the three types of Independent Variables.

"Effective return" requires explication. First, a boy in the seventh grade had a brother in the eighth grade. Both the mother and the father of the brothers responded to the survey request. One boy's return was paired with the father's return and the brother's return was paired with the mother's return. Other examples include a brother and sister in the eighth grade, twin girls in the eighth grade, and a girl in the seventh grade who had a brother in the eighth grade. However, for these three sets, only the mother supplied a return, so her return was utilized twice, paired with each of the siblings. Thus, the return $N$ of 76 is "effective" rather than literal. Arguably, these "effective" duplicate scores from within the same family could be dropped. But, because of the nature of the study and because of the overall small return rate, the decision was made to include them with the adjustments as noted.

For all of the data, a spreadsheet was created. Missing data for each variable were then coded as the mean or modal response for that category, depending on level of measurement. This action is a conservative correction.

\section{Independent Variables}


The Independent Variables for this study include Demographic Controls, Parent Values, and Parent Involvement. The descriptive statistics for those constructs are presented in turn.

\section{Demographic Controls}

The Demographic Controls are the three constructs: Family Structure (FS), Socioeconomic Status (SES), and Specially Designed Instruction (SDI).

Family Structure. Questions on the Family Structure (FS) portion of the survey were designed to establish a picture of the family unit and of the resources potentially available to them as related to a child's educational achievement. In addition to questions concerning the number of children and adults residing in the home, the survey instrument included questions such as those concerning number of generations represented by the family unit, the availability of transportation to family members, and the duration of Parent Involvement over extended generations. However, because of the comprehensive meaning of child-to-adult ratio, and for the purpose of economizing space, the decision was made to represent the "effective" child/caretaker ratio as the Family Structure (FS).

Table 3 reflects the Child/Caretaker ratio derived from the survey. Children living in the household who were currently enrolled in school are counted in Parent Survey Question V A 4. Children living in the household who were not yet enrolled in school are counted in Parent Survey Question V A 5. These two questions are combined to yield Dependent Children. Other people living in the household who were dependent on family resources due to their physical or mental disability were numbered through Parent Survey Question V A 7.

The number of adult parents and other caretakers in the household comes from 
Parent Survey Question V A 6. Adding the Dependent Children plus Disabled Adults and then dividing by the number of Adult Caretakers provides a ratio scale on which high scores indicate fewer parental resources available. This seemingly inverted scale is unavoidable because, in a large population, an undefined domain would occur for a single adult or couple with no dependents ( 0 in denominator). Thus, the FS score, ratio of dependent children plus disabled adults in the home divided by adult caretakers, is $M=$ 1.05 , meaning that for those answering the survey, number of caretakers almost equals dependents.

\section{Table 3}

Descriptive Statistics for Family Structure $(N=38)$

\begin{tabular}{lccc}
\hline \multicolumn{1}{c}{ Measure } & $M$ & $S D$ & Range \\
\hline Dependent Children & 2.16 & 1.63 & $1-10$ \\
Disabled Adults in the home & .05 & .23 & $0-1$ \\
Adult Caretakers & 1.90 & .31 & $1-2$ \\
Family Structure Score & 1.05 & .42 & .50 \\
\hline
\end{tabular}

Note. The Family Structure Score is the ratio of Dependent Children plus Disabled Adults in the home divided by Adult parents or other caretakers.

Socioeconomic Status. To establish an SES score for participants, answers provided to the Demographics (SES) section of the Parent Survey were assigned point values (see Appendix Q). The question on level of education represents a six-point ordinal scale, although the response categories are not far from being interval. The 
section on family income is a six-point interval scale. Table 4 gives frequency and percentage of parents for the different categories under educational attainment and income level.

Table 4

Descriptive Statistics for Socioeconomic Status Factors $(N=38)$

\begin{tabular}{lccc}
\hline Category & Level & Frequency & $P^{a}$ \\
\hline & Education Level & & \\
$8^{\text {th }}$ & 1 & 1 & 3 \\
$9-11$ & 2 & 3 & 8 \\
$12^{\text {th }}$ & 3 & 14 & 37 \\
Some college & 4 & 13 & 34 \\
College graduate & 5 & 3 & 8 \\
Graduate school & 6 & 4 & 11 \\
\hline
\end{tabular}

Family Income Level

\begin{tabular}{llll}
$\$ 0-15,000$ & 1 & 2 & 5 \\
$\$ 15,001-30,000$ & 2 & 1 & 3 \\
$\$ 30,001-45,000$ & 3 & 9 & 24 \\
$\$ 45,001-60,000$ & 4 & 16 & 42 \\
$\$ 60,001-75,000$ & 5 & 5 & 13 \\
$\$ 75,001-90,000+$ & 5 & 13 \\
\hline Note. Education Level represents highest level completed. & \\
${ }^{\text {a Percentages do not sum to } 100 \text { because of rounding. }}$
\end{tabular}


Table 4 indicates that levels 3 and 4 (high school graduate and some college) are the most common educational attainment. The income range of $\$ 45,001-\$ 60,000$ was the most frequent. To establish the SES score, the interval income levels and ordinal education levels were combined, adding the points (levels) for the two respective indicators of SES. This converts the two scales into an overall derived SES score based on an interval scale, with possible scores from 2-12. The actual range was between 5 and 12 SES points. The mean for the effective return of 38 families on this derived SES scale was 8.05 with a standard deviation of 1.87 .

Specially Designed Instruction. Known as Specially Designed Instruction (SDI), the special education unit of DEMS enrolls students determined to be learning disabled, emotionally disabled, or educationally challenged. Participants in the SDI program were assigned 0 points; non-participants were assigned 1 point in order to reflect a higher academic status. Only five students included in the surveys returned were SDI participants (13.2\%); the remaining $33(86.8 \%)$ students were in the regular classroom. Parent Values

The second type of independent variable included in this study is Parent Values. These are alterable factors under Bloom's (1980) scheme, although for most parents, these values (as adults) would be relatively stable. However, events or new information (e.g., taking classes, a revival) could conceivably result in changes in value positions. Two sets of Parent Values are included: Rural Values and Parent Religiosity. For both sets of Parent Values and the mediating variable, Parent Involvement, responses to scales were collected in parallel from parents and students. Because the answers from students were matched to their parents, it was possible to compute descriptive statistics for 
parents, for students, and for parents and students combined.

Rural Values. Five different Rural Values were developed, based on the literature and the author's lifelong experience in a southern rural community. Table 5 presents the results of the responses to survey questions designed to measure these five Rural Values. Responses were based on between five to seven items for each construct, measured by a 5-point Likert scale with $5=$ high. Scale items for the different Rural Values are given in Appendix T. The results indicate that Stewardship of Earth and Ethnocentricity were scored highest and Anti-Intellectualism was scored lowest by both parents and students. After checking scale reliabilities (see Psychometric Analysis of Scales, below), one from each of the following constructs was deleted: Anti-Intellectualism, Ethnocentricity, and Stewardship of Earth. Table 5 reflects those revisions.

Table 5

Summary Descriptive Statistics for Rural Values $(N=38)$

\begin{tabular}{|c|c|c|c|c|c|c|}
\hline \multirow[b]{2}{*}{ Construct } & \multicolumn{2}{|c|}{ Parent } & \multicolumn{2}{|c|}{ Student } & \multicolumn{2}{|c|}{ Combined } \\
\hline & $M$ & $\overline{S D}$ & $\bar{M}$ & $S D$ & $M$ & $S D$ \\
\hline Anti-Intellectualism & & & & & & \\
\hline & 2.55 & .85 & 3.07 & .28 & 2.81 & .51 \\
\hline Social & & & & & & \\
\hline $\begin{array}{l}\text { Conservatism/ } \\
\text { Traditionalism }\end{array}$ & 2.60 & 1.08 & 2.60 & .94 & 2.87 & .92 \\
\hline Ethnocentricity & 4.00 & .65 & 3.65 & .21 & 3.80 & .43 \\
\hline $\begin{array}{l}\text { Stewardship of } \\
\text { Earth }\end{array}$ & 4.07 & .12 & 4.23 & .10 & 4.16 & .05 \\
\hline Good Old Boy & 2.66 & .71 & 3.19 & .26 & 2.93 & .44 \\
\hline
\end{tabular}


Parent Religiosity. The set of Parent Religiosity values includes five constructs. First, sixteen questions assessed Belief (PR-BEL), which was divided into three subconstructs: School as Threat used five items, Integration of Religion into School had five, and Literalist had six. These subscales were not analyzed separately for this study but could be used in further investigations. Belief was measured by a 5-point Likert scale. Second, the responses to eleven items of a behavior frequency scale, from $1=$ "seldom" to 7 = "more than once daily," established the Ritual score (PR-RIT). Third, six questions assessed religious Experience (PR-EXP), utilizing a 5-point Likert scale (5 as high). Fourth, Primary Affiliation (PR-PAFF) was assessed through answers to five items in each of the following areas: church, school, and other. The response scale was a 5-point Likert-type from "no chance" to "excellent chance of choosing this group" ( $5=$ excellent). Fifth, Affiliation Bond (PR-ABOND) was determined for school, church, civic organizations, and other. Respondents chose from a 9-point "dilemma" scale that required them to rate their feelings of being "included": e.g., as outsider or insider, audience or participant. Response options ranged from 7 at the less involved pole of the scale, to 3 (neutral), and then up to 7 at the more involved end of the pole. Points were assigned progressively from the less involved end: e.g., "less involved 7" $=1,3=5$, and "more involved 7" $=9$.

Table 6 summarizes the results. Answers for the PR-BEL, PR-EXP, and PRPAFF ranged from 1-5, so the $M$ for these scales were on the high side. Experience gained the highest score of the three constructs measured by the 5-point Likert scale for both parents $(M=3.86)$ and students $(M=3.75)$. PR-RIT was measured on a 1-7 scale; therefore, the mean for parents and students were toward the less active end. Likewise, 
the PR-ABOND scores were reflected by a 9-point scale, so the combined $M=5.69$ for parents and students reflected PR-ABOND of only slightly "more involved."

Table 6

Descriptive Statistics for Parent Religiosity $(N=38)$

\begin{tabular}{|c|c|c|c|c|c|c|}
\hline \multirow[b]{2}{*}{ Construct } & \multicolumn{2}{|c|}{ Parent } & \multicolumn{2}{|c|}{ Student } & \multicolumn{2}{|c|}{ Combined } \\
\hline & $M$ & $S D$ & $M$ & $S \overline{S D}$ & $M$ & $S D$ \\
\hline Belief & 3.70 & .88 & 3.68 & .69 & 3.69 & .75 \\
\hline Ritual $^{\mathrm{a}}$ & 3.55 & 1.12 & 3.38 & 1.14 & 3.45 & 1.12 \\
\hline Experience & 3.86 & .69 & 3.75 & .58 & 3.80 & .63 \\
\hline Primary Affiliation & 3.45 & .43 & 3.50 & .47 & 3.51 & .43 \\
\hline Affiliation Bond ${ }^{b}$ & 5.41 & .63 & 6.05 & .56 & 5.69 & .51 \\
\hline
\end{tabular}

Note. Scale items and scoring of responses are provided in Appendix T.

${ }^{a}$ Ritual is based on a 7-point scale.

${ }^{\mathrm{b}}$ Affiliation Bond is based on a 9-point scale.

\section{Parent Involvement}

Parent Involvement represents the third type of independent variable in this study, Mediating Variables (see Figure 1). The model postulates that the degree of parental involvement "bridges" parent values and students' achieved grades, i.e., the greater the parents' involvement in school, the more basic values will be reinforced to the children and the more students will invest in their studies. Parent Involvement includes two variables. First, with five questions, the surveys explored Belief (in parent involvement), measured by a 5-point Likert scale. Second, Action used a 5-point behavioral frequency scale that asked for responses to frequencies from Never $=1$ to Always $=5$. Thirteen 
items were included in this scale. Table 7 summarizes the findings. The means for Belief are higher than Action for all three levels of data.

Table 7

Descriptive Statistics for Parent Involvement $(N=38)$

\begin{tabular}{|c|c|c|c|c|c|c|}
\hline \multirow[b]{2}{*}{ Construct } & \multicolumn{2}{|c|}{ Parent } & \multicolumn{2}{|c|}{ Student } & \multicolumn{2}{|c|}{ Combined } \\
\hline & $\bar{M}$ & $S D$ & $M$ & $S D$ & $M$ & $S D$ \\
\hline Belief & 3.97 & .38 & 3.47 & .31 & 3.72 & .27 \\
\hline Action & 3.21 & .59 & 2.78 & .53 & 3.00 & .55 \\
\hline
\end{tabular}

Note. Scale items are listed in Appendix T.

\section{Dependent Variables}

The dependent variable for this study is Student Outcomes as revealed through Grade Point Average (GPA), a percentage measurement. The student level data were collected from the guidance counselor at the high school where the students attended after leaving Dixon Elementary Middle School. For this sample the mean GPA is 86.66 with a standard deviation of 8.30 and a range of $68 \%-98 \%$.

Psychometric Analysis of Scales

The scales in the Parent Values and Parent Involvement columns of Figure 1 were developed expressly for this study, based on items adapted from the literature and additional questions composed by the author, tapping her knowledge of and experience in education and rural life. Because these scales have not been previously used, information on their psychometric properties is needed. In addition, because the case study is so limited in terms of subjects, possibilities of data reduction are relevant.

Nitko (2001) notes that concepts of validity and reliability of measurement tools 
have changed from traditional approaches. In the past, these characteristics were believed to be inherent to the instrument itself. The current understanding is that validity depends upon the particular way in which a measurement is used and interpreted, including information about the contextual setting. Nitko discusses eight separate types of validity evidence. The limitations of this case study preclude addressing each of these. Some information is available for the issues of content evidence, internal structure evidence, external structure evidence, reliability evidence (now subsumed as one type of validity data as opposed to the separation of reliability and validity under traditional psychometric thinking), and generalization evidence. Comments on the other types of validity evidence are included to the extent possible.

According to Nitko (2001), validity refers to the interpretations an author makes based on the data that are collected, not to the instrument used. An assessment of the validity of the interpretations of results should be made only after considering several types of validity within the study such as how data are used and the value that readers impose on those interpretations. Once the assessment is made, a researcher must form a convincing argument, based on those considerations, to validate the study.

Nitko (2001) states that previously validity was considered in three forms: content, criterion, and construct. However, measurement specialists now hold these as types of evidence that support a unitary concept of validity. The information that follows is organized by the type of validity evidence. The five types of evidence available in this study are addressed in turn, followed by a section on the remaining types of validity to the extent applicable to this case study. Within each section, the data are arranged according to the type of independent variable: alterable (Parent Values) and mediating 
(Parent Involvement) (see Figure 1).

\section{Content Evidence Validity}

The literature review for this study included much evidence to support constructs addressed in this study. In the area of Rural Values-Anti-Intellectualism, the work of Hofstadter (1963) was critical. Little (1998) provided a background for the Social Conservatism/Traditionalism and Ethnocentricity scales. Stewardship of Earth was derived from work by Ching \& Creed (1997), Howley \& Howley (1995), Verhoog (1995), Verkleij (1995), Witte (1995), and Zweers (1995). (1997) and Hemphill (1974) provided a great deal of the groundwork for Good Old Boy. Other scholars doing work on rural values were Ching and Creed (1997), Din (1998), and Herzog and Pitman (1995). In addition, Dr. Dwight Cline from Western Kentucky University provided source materials for the constructs in Rural Values.

Parent Religiosity construct foundations were laid in works by Glock (1973) and Stark (1999) concerning Belief, Ritual, and Experience. Moreover, the author has had twenty years experience as an active member of the conservative Baptist community. Darnell and Sherkat (1997), Ellison and Sherkat (1993), and Sherkat and Darnell (1999) provided numerous studies to lend validation in the Ritual and Affiliation areas. Affiliation Bond as a construct is supported most by Asa's (1995) work and by work on the fundamentalist class proffered by Coreno (2002).

Joyce Epstein's (1995) studies in Parent Involvement provided valuable assistance in constructing the educational paradigms for this area. Maxim and Five (1997) and Meade (1992) offered further support for the content in these constructs.

In addition to the issue of consistency of content to existing literature, the twelve 
scales (five Rural Values, five Parent Religiosity, and two Parent Involvement) were subjected to review by two experts in the area of rural education. Dr. Paula Little (1998) created and validated four rural values in her study of hiring practices in rural school districts. Three of the scales in this study were adapted from her work. Dr. Dwight Cline has a lifetime of experience in rural education and taught classes in rural education policy and educational administration. Both critiqued the scales for content and conceptual integrity.

Dr. Darren Sherkat, a widely published author and scholar of sociology of religion at Southern Illinois University, read sections of the scales dedicated to religiosity and provided feedback to their validity. Dr. Christopher Wagner, instructor at Western Kentucky University and a nationally known consultant in (and a strong advocate of) Parent Involvement, provided valuable feedback in the execution of this study.

Finally, the author had her English 102 class at a local community college critique the scales for content issues. These students are lifelong residents of small towns and rural Kentucky. Feedback from these sources were incorporated into scale revisions.

\section{Internal Structure Evidence Validity}

The internal structure validity can be examined once the interrelationships of the tasks in the study and its results are established, particularly for a study including educational constructs, as in this case. How well the different assessment tasks work together, how consistent the assessment is with the theory upon which the study is based, and how the scores of the separate parts of the assessment are inter-correlated support the internal structure evidence. These construct inter-correlations can be measured by Pearson $r$ coefficients. 


\section{Data Reduction Procedures}

Based on Figure 1, the theoretical model, there are two blocks of Parent Values: five Rural Values and five Parent Religiosity. In addition, the mediating variable Parent Involvement has two scales. This totals twelve separate constructs. But data were collected in parallel from both parents and students, yielding a total of 24 separate variables: twelve parent and twelve student. In the current study, constrained by a very small $N$, efforts were taken to reduce the amount of data, from 24 separate variables to a smaller, more manageable number of variables. These calculations were performed prior to constructing the Pearson $r$ correlation matrix.

The first task was to determine whether the parent response and the student response for each separate scale could be combined into a single variable. The procedure followed was to calculate Cronbach's (1951) coefficient alpha for the parent, student, and combined variables. Based upon these results, an "optimal" variable for each of the twelve scales was selected. In order to be consistent, results were analyzed according to a set of selection rules.

Data Reduction Rules. Rules for reduction of data were formulated to compare the twelve independent variables for the three levels. Cronbach's (1951) Coefficient alpha scores for the Parent, Student, and Combined scales were then scrutinized according to the following criteria:

Rule \# 1. The combined scale was used if its coefficient alpha met the accepted standard of .7 for scale reliability.

Rule \# 2. If the scale did not meet the 7 criterion, Nunnally and Bernstein's (1994) relaxed criterion of .6 or better was accepted, consistent with exploratory research.

Rule \#3. The combined scale was used whenever it was as good as or slightly less than 
the better of the parent or student coefficient alpha.

Rule \# 4. In case of a large discrepancy between coefficient alpha for the parent and student scales, when the lower of these two alphas was extremely low, producing a situation in which the combined alpha score was intermediate (between Low and High for Parent or Student), then the higher of the two (parent or student) coefficient alpha was utilized and data for the extremely low value was discarded. The rationale here was that for whatever reason, the scale items for the parents (or students) apparently did not make sense and/or respondents did not perceive the items as sufficiently similar to comprise a coherent scale.

Rule \# 5. If a single item within a scale was detected to have a major effect on the alpha, and the preliminary Reliability Analysis indicated that its removal from the scale would raise the alpha score by a considerable degree (alpha with item deleted), the single item was deleted from each individual scale (Parent, Student, and Combined), and the scale was revised to reflect the deletion.

The initial calculations are shown for the individual scale items of each of the twelve variables in Appendix T, Tables T1-T12. Table 8 summarizes Tables T1-T12 based upon the twelve independent variables. Cronbach's alpha scores for Parent, Student, and Combined are presented for the five Rural Values (RV), five Parent Religiosity (PR), and two Parent Involvement (PI) scales. These results reflect the Cronbach's alpha for each scale, based upon the original number of items in each construct.

Inspection of Tables T1-T12 reveals that the Cronbach's (1951) alphas for the five scales under Parent Religiosity and two scales for Parent Involvement were the optimal values. Specifically Tables T6-T12 demonstrate that the originally conceived scales represent either the highest internal reliability possible for those constructs, or that the gain in the alpha level by deleting on item is so minimal that the integrity of the a priori set of content items would be destroyed for no empirical gain in measurement precision. 
Table 8

Initial Coefficient Alphas for Parent Values and Parent Involvement Constructs $(N=$ 38)

\begin{tabular}{|c|c|c|c|}
\hline \multirow[b]{2}{*}{ Variable } & \multicolumn{3}{|c|}{ Coefficient Alpha } \\
\hline & Parent & $\overline{\text { Student }}$ & Combined \\
\hline \multicolumn{4}{|c|}{ Rural Values } \\
\hline Anti-Intellectualism & .424 & .630 & .635 \\
\hline $\begin{array}{l}\text { Social } \\
\text { Conservatism/Traditionalism }\end{array}$ & \multicolumn{2}{|c|}{ Conservatism/Traditionalism } & .527 \\
\hline Ethnocentricity & .635 & .438 & .571 \\
\hline Stewardship of Earth & .647 & .606 & .622 \\
\hline Good Old Boy & .376 & .789 & .620 \\
\hline \multicolumn{4}{|c|}{ Parent Religiosity } \\
\hline Belief & .762 & .865 & .854 \\
\hline Ritual & .869 & .909 & .912 \\
\hline Experience & .676 & .819 & .801 \\
\hline Primary Affiliation & .925 & .964 & .962 \\
\hline Affiliation Bond & .908 & .916 & .921 \\
\hline \multicolumn{4}{|c|}{ Parent Involvement } \\
\hline Belief & .675 & .752 & .735 \\
\hline Action & .878 & .837 & .867 \\
\hline
\end{tabular}

Note. Table is derived from Appendix T, Tables T1-T12.

In contrast, the situation for the five Rural Values scales is more complex. For 
Anti-Intellectualism, one item seemed to work adversely with the other items in the scale:

II. 3, "Rural schools need strong sports programs." That item was removed and the Combined alpha for RV-AI rose to .714. No change was made in the Social Conservatism/Traditionalism (RV-SC/T) items because the items seemed to be scored consistently within the Parent, Student, and Combined results. The Student reliability alpha (.072) was extremely low, however, and caused the Combined alpha to drop to .527. The Parent data (alpha $=.655)$ were used for subsequent analysis because the student data were not meaningful. The Rural Values-Ethnocentricity alpha scores were originally Parent $($ alpha $=.635)$, Student $($ alpha $=.438)$, and Combined $($ alpha $=.571)$. After deleting survey item II. 16, “'City' teachers who come here don't understand country people," a Parent alpha of .662 was established for a five-item scale that met the relaxed criterion of .6 (Nunnally \& Bernstein, 1994); again the student data yielded internal reliability so low that these responses were dropped. Likewise, one item in the Rural Values-Stewardship of Earth (SE) appeared to weaken the scale: II. 30, "A farmer's right to farm his own land can be taken away by environmentalists who promote "wetland' protection." That item seemed to conflict with the remaining SE scale items; it was removed and a new variable was created, increasing the overall Combined alpha to .837. Finally, it appeared that parents did not respond consistently to items in the Rural Values-Good Old Boy section of the survey (Parent alpha $=.376)$. The parent data were dropped and the original GOB scale was maintained based on Student data (alpha = .789). Thus for the five Rural Values scales, the following changes were made. For Anti-Intellectualism, one item was removed and the Combined data were utilized. For Social Conservatism/Traditionalism, the original scale was maintained, but the Student 
responses were dropped, leaving Parent only data. For Ethnocentricity, one item was eliminated and Student responses were again eliminated, leaving a Parent only scale. For Stewardship of the Earth, one item was removed and the Combined data were optimal. Finally, for Good Old Boy, the original scale was maintained but the Parent responses were dropped, leaving a Student only scale. (The reliability analysis for the three scales in which an item was eliminated--Anti-Intellectualism, Ethnocentricity, and Stewardship of Earth--appear as Tables T13-T15, respectively.)

Summing up, Table 8 presents the coefficient alpha values for the three blocks of scales (Rural Values, Parent Religiosity, and Parent Involvement) based on the original set of reliability analyses (Tables T1-T12). Results for Parent, Student, and Combined levels of data are shown. Similarly, Table 9 presents the coefficient alpha values for the three Rural Values scales in which an item was deleted, based on Tables T13-T15.

Table 9

Optimal Coefficient Alphas for Rural Values Constructs with Item Deleted $(N=38)$

Variable

\begin{tabular}{ccc}
\multicolumn{3}{c}{ Coefficient Alpha } \\
\hline Parent & Student & Combined
\end{tabular}

Anti-Intellectualism

.443

.725

.714

Ethnocentricity

.662

.494

Stewardship of Earth

.811

.816

.837

Note. Table is derived from Appendix T, Tables T13-T15.

Table 10 reveals the optimal scales for further analyses based on the reliability analyses in Tables T1-T15 and consistent with the Data Reduction Procedures listed 
above. Finally, all except two (Social Conservatism/Traditionalism and Ethnocentricity) met the standard criterion of .7 or above for internal reliability; both met the relaxed standard of .6 (Nunnally \& Bernstein, 1994) deemed adequate for exploratory research.

Table 10

Optimal Derived Scales Based on Reliability Analysis

Variable

Scale

Coefficient Alpha

\section{Rural Values}

Anti-Intellectualism

Combined

.714

Social

Parent

.655

Conservatism/Traditionalism

Ethnocentricity

Parent

.662

Stewardship of Earth

Combined

.837

Good Old Boy

Student

.789

Parent Religiosity

Belief

Combined

Ritual

Combined

Experience

Combined

Primary Affiliation

Combined

.962

Affiliation Bond

Combined

.921

\section{Parent Involvement}

Belief

Combined

Action

Combined

.867 
Further data reductions. Table 10 summarizes the optimal scale for each of the twelve constructs. A further question can then be asked. Can the five scales in Rural Values be combined into an overall Rural Values construct? Similarly, this can be asked of Parent Religiosity and Parent Involvement. Thus the scales were subjected to further analysis to see if the constructs could be combined into one overall variable for $R V, P R$, and PI.

Table T16 in Appendix T gives the reliability analysis of these three overall constructs. The Rural Values scale proved problematic; the Rural Values constructs did not seem to hold together as a scale. Accordingly the optimal scales for these five constructs were used, one at a time, as independent variables: Anti-Intellectualism, Social Conservatism/Traditionalism, Ethnocentricity, Stewardship of Earth, and Good Old Boy, as listed in Table 10. Coefficient alpha scores of the Parent Religiosity and Parent Involvement constructs supported reduction to two overall variables: (a) Belief, Ritual, Experience, Primary Affiliation, and Affiliation Bond were reduced to PR, (b) Belief and Action were reduced to PI. Table 11 summarizes those calculations. 
Table 11

Final Data Reduction Scales

\begin{tabular}{lccc}
\hline Variable & $M$ & $S D$ & alpha \\
\hline Anti-Intellectualism & 2.81 & .51 & .725 \\
Social Conservatism/Traditionalism & 2.78 & 1.08 & .655 \\
Ethnocentricity & 4.00 & .65 & .662 \\
Stewardship of Earth & 4.16 & .05 & .837 \\
Good Old Boy & 3.19 & .26 & .789 \\
Parent Religiosity & 4.01 & .95 & .777 \\
Parent Involvement & 3.36 & .51 & .751 \\
\hline
\end{tabular}

\section{Pearson r Inter-correlations}

Based upon the completed data reductions, it is now possible to examine intercorrelations among the retained Parent Values (Rural Values and Parent Religiosity) and Parent Involvement scales. As indicated above, these provide information on internal structure validity.

Table 12 presents the inter-correlations. Variables 1-5 represent the five Rural Values scales. The inter-correlations are generally not significant, ranging from $r=-.006$ to .299 , with two exceptions. Anti-Intellectualism correlates with Good Old Boy $(r=$ $.421, p<.01)$ and Ethnocentricity is associated with Stewardship of Earth $(r=.462, p<$ $.01)$. This indicates that these five Rural Values have essentially no internal structure as a set of constructs, although the two individual connections are moderately strong. 
Variables 6-10 represent the five Parent Religiosity constructs. All but one of these inter-correlations are significant, ranging from a low for the association of Belief with Affiliation Bond $(r=.304)$ to a high for Primary Affiliation with Affiliation Bond ( $r$ $=.665, p<.01)$. These results suggest considerable internal connections for the five scales.

Finally, the two Parent Involvement scales2, Belief and Action, are correlated at $r$ $=.601, p<.01$, indicating a substantial internal structure for Parent Involvement. 
Table 12

Inter-Correlation Statistics for Optimum Parent Values and Parent Involvement Scales $(N=38)$

\begin{tabular}{|c|c|c|c|c|c|c|c|c|c|c|c|c|}
\hline & 1 & 2 & 3 & 4 & 5 & 6 & 7 & 8 & 9 & 10 & 11 & 12 \\
\hline $1^{\mathrm{a}}$ & -- & & & & & & & & & & & \\
\hline $2^{b}$ & .275 & -- & & & & & & & & & & \\
\hline $3^{b}$ & -.006 & .151 & -- & & & & & & & & & \\
\hline $4^{a}$ & .018 & .299 & $.462 * *$ & -- & & & & & & & & \\
\hline $5^{c}$ & $.421^{* *}$ & .101 & -.206 & .079 & -- & & & & & & & \\
\hline $6^{\mathrm{a}}$ & .301 & .037 & .260 & $.472 * *$ & .158 & -- & & & & & & \\
\hline $7^{\mathrm{a}}$ & .077 & -.153 & .109 & .272 & -.110 & $.548^{* *}$ & -- & & & & & \\
\hline $8^{a}$ & .178 & .011 & $.384^{*}$ & $.602 * *$ & .133 & $.610^{* *}$ & $.609^{* *}$ & -- & & & & \\
\hline $9^{a}$ & -.079 & .130 & $.438^{* *}$ & $.561^{* *}$ & -.046 & $.425^{* *}$ & $.372^{*}$ & $.592^{* *}$ & -- & & & \\
\hline $10^{\mathrm{a}}$ & .142 & .056 & $.396^{*}$ & .277 & -.058 & .304 & $.372^{*}$ & $.411^{*}$ & $.665^{* *}$ & -- & & \\
\hline $11^{\mathrm{a}}$ & -.142 & .053 & .215 & .304 & -.097 & .159 & $.242^{*}$ & .252 & $.414^{*}$ & $.373^{*}$ & -- & \\
\hline $12^{\mathrm{a}}$ & .071 & .017 & $432 * *$ & $.521^{* *}$ & -.042 & $.373^{*}$ & .160 & $.417^{*}$ & $.524^{* *}$ & $.528 * *$ & $.601 * *$ & -- \\
\hline
\end{tabular}


Table 12 (continued)

Note. 1 = RV-AI; 2 = RV-SC/T; 3 = RV-E; $4=$ RV-SE; $5=$ RV-GOB; $6=$ PR-BEL; $7=$ PR-RIT; $8=$ PR-EXP; $9=$ PR-

PAFF $; 10=$ PR-ABOND; $11=$ PI-BEL; $12=$ PI-ACT.

${ }^{a}$ Scale is based on Parent and Student Combined responses.

${ }^{\mathrm{b}}$ Scale is based on Parent only responses.

${ }^{\mathrm{c}}$ Scale is based on Student only responses.

${ }^{*} p<.05 .{ }^{* *} p<.01$. 


\section{Table 13}

Correlation Statistics for Parent Values (Rural Values and Parent Religiosity) and Parent Involvement Based on Optimal Coefficient Alpha $(N=38)$

\begin{tabular}{|c|c|c|c|c|c|c|c|}
\hline & 1 & 2 & 3 & 4 & 5 & 6 & 7 \\
\hline $1^{\mathrm{a}}$ & -- & & & & & & \\
\hline $2^{b}$ & .314 & -- & & & & & \\
\hline $3^{b}$ & .119 & .151 & -- & & & & \\
\hline $4^{\mathrm{a}}$ & .125 & .311 & $.478^{* *}$ & -- & & & \\
\hline $5^{\mathrm{a}}$ & $.414^{* *}$ & .101 & -.206 & .078 & -- & & \\
\hline $6^{\mathrm{a}}$ & .279 & .007 & $.395^{*}$ & $.509^{* *}$ & .010 & -- & \\
\hline $7^{\mathrm{a}}$ & .113 & .039 & $.364^{*}$ & $.405^{*}$ & -.077 & $.493^{* *}$ & -- \\
\hline \multicolumn{8}{|c|}{ Note. 1 = RV-AI; $2=\mathrm{RV}-\mathrm{SC} / \mathrm{T} ; 3=\mathrm{RV}-\mathrm{E} ; 4=\mathrm{RV}-\mathrm{SE} ; 5=\mathrm{RV}-\mathrm{GOB} ; 6=\mathrm{PR}$} \\
\hline \multicolumn{8}{|c|}{${ }^{\text {a }}$ Scale is based on Parent and Student Combined responses. } \\
\hline \multicolumn{8}{|c|}{${ }^{\mathrm{b}}$ Scale is based on Parent only responses. } \\
\hline \multicolumn{8}{|c|}{ ' $\mathrm{c}$ cale is based on Student only responses. } \\
\hline & $<.01$ & & & & & & \\
\hline
\end{tabular}

Table 13 presents the inter-correlations for the final set of Parent Values (Rural Values and Parent Religiosity) and Parent Involvement, based on final data reductions, as summarized in Table 11. Variables 1-5 are the separate Rural Values constructs while variable 6 is the overall Parent Religiosity and variable 7 is the overall Parent Involvement. Parent Religiosity is significantly related to two Rural Values-Ethnocentricity and Stewardship of Earth. Parent Involvement is significantly related to 
the same two Rural Values. Parent Religiosity is significantly related to Parent Involvement $(r=.493, p<.01)$. Thus the internal structure connections among those four variables are moderate, in addition to the two significant correlations among the five Rural Values constructs (Table 12 above).

\section{External Structure Evidence Validity}

Construct validity has been, traditionally, the focus in External Evidence Structure. The fact that this is a case study limits gathering any additional evidence to support the constructs herein; however, some evidence for construct validity does exist. Content relevance (see above) has been established in the review of the literature according to the developer's domain. The data just examined under internal structure evidence (above), as well as evidence for the internal reliability of the scales (see Data Reduction Procedures and related sections, above), demonstrate the coherence of the constructs internally and their moderate inter-correlation with one another, consistent with the theoretical model. These results confirm that the constructs group into three subsuming blocks--Rural Values, Parent Religiosity, and Parent Involvement. Other conditions of external structure evidence deal with the results of the assessment and their relationships to specified criteria.

No data are available for predictive validity (case study, not longitudinal in design). However evidence on concurrent validity is inherent in the answers to the research questions. Those answers establish what has been traditionally referred to as "criterion validity"; for this case study this is the most important piece of confirming/ disconfirming evidence on the overall validity of the scales. Criterion evidence depends on how well the results are correlated with relevant criteria. In this case, the relationship 
between Parent Values (Rural Values and Parent Religiosity) and Parent Involvement is investigated in Research Question 5 (see below). The more direct criterion measure is how these independent variables (Parent Values and Parent Involvement) affect student GPA, the outcome measure in this study of parent involvement at the middle school. Research Questions 4 and 6 address that issue directly (see below).

\section{Reliability Evidence Validity}

The consistency of an assessment's results establishes Reliability Evidence Validity. Three basic types of reliability exist: consistency over time, internal consistency across content items, and consistency across raters. For this study only the internal reliability of the items within a scale is pertinent, i.e., how consistently the instrument measures content representing a sample of the possible items for a particular topic. As already described above under Data Reduction Procedures, Cronbach's (1951) coefficient alpha was conducted for each of the twelve Parent Values and parent Involvement scales. The final results of these reliability analyses are summarized in Table 11. Those findings confirm that the scales in this study have acceptable internal reliability, based on their final form following psychometric data reductions.

\section{Generalization Evidence Validity}

Generalization Evidence is interpretation of results for assessments conducted with different kinds of people under other circumstances. An assessment is said to have Generalization Evidence Validity if results are consistent despite changes in people, situations, special interventions, or occurrences during the administration of the assessment, i.e., if the same results are obtained regardless of who is assessed, where, when, and how. 
For this case study, generalization evidence can be used to guide the data reduction decisions, i.e., Parent and Student data are combined to the extent that these responses can be generalized over generations. The results indicate that for nine of the twelve Parent Values and Parent Involvement Scales, the data do generalize across generations. The case study design limits generalization directly. Further evidence on generalization validity evidence must await subsequent research to extend these case study findings.

\section{Other Types of Validity Evidence}

Substantive evidence may be indirectly obtained during the administration of the assessment under other circumstances when participants reveal the skills and processes they utilized in responding to the instrument. During the piloting of this study, an English 102 community college class commented on the difficulty of answering some items and the potential for responding in several ways to others. Also, comments were made about the fact that the assessment caused them to think about their values and culture in ways they never had before. However, the data to support this are suggestive at best.

Consequential Evidence relates to possible effects that the assessment might have on the participants in the study. In addition to the evidence of consequential outcomes mentioned above, several incidents provided possible evidence of the consequential realm. First, upon responding to the request for the author to administer the student assessment within the classroom setting of a teacher at the school, the assistant superintendent indicated that she felt a questionnaire concerning values was inappropriate for a middle school audience. She also felt that any reference of any kind to the word 
religion might generate bad reactions or animosity toward the school system. Based on that hypothesis, she refused to allow the assessment to be conducted at school. In addition, despite the author's previous employment and disability leave from the school under study, she was refused access to student addresses out of fear of negative consequences. (The telephone directory was subsequently used to secure addresses.) Second, some of the interviewees commented on how thought provoking, revealing, and "deep" they believed the questions to be. Most of them voiced their appreciation at being included in the study. Third, one student (8ML) called to confirm the actual possibility of the study being published. He stated he thought the study made some important points, was very intriguing, and should be read by every school administrator in the system because "it might change some things." $8 \mathrm{ML}$ student told of his concern for protecting and preserving his rural heritage for his children; he thought that the school system was anti-rural: "They need to know how important things like hunting and working on the farm are. None of them really care about us kids and what is important to us." Again, any evidence in this realm is based on anecdotal feedback.

Finally, Practicality Validity relates to the cost and effort involved in the assessment procedures. During the piloting phase of the study, some respondents were discouraged by the time it took to complete the survey. As a result the survey was shortened, and the next group of pilot participants completed the survey within twenty minutes; no one complained about the time it took. However, despite the inclusion of a self-addressed stamped envelope and a dollar, because the survey had to be administered by mail, response rate was drastically reduced from 109 to 38 . This circumstance reduced significantly the power of the data analysis and overall validity considerations. 


\section{Research Questions}

In order to answer the research questions and to detect the magnitude of the observed effects, statistical significance and effects are estimated via multiple regression. The stepwise procedure is utilized for Research Questions 1-6 while hierarchical regression is performed for Research Questions 7-10. The data utilized for the Parent Values and Parent Involvement scales were based on the psychometric analyses in the previous section. For the overall Parent Religiosity and Parent Involvement constructs, parent and student combined responses comprised the data. For the five Rural Values scales, the locus of the data--Parent, Student, or Combined--is based on the psychometric work: Anti-Intellectualism (Combined responses), Social Conservatism/Traditionalism (Parent responses), Ethnocentricity (Parent responses), Stewardship of Earth (Combined responses), and Good Old Boy (Student responses). Per discussions in Chapter III, a relaxed standard for significance was chosen (alpha $=.10)$

Table 14 presents the correlation matrix upon which the multiple regressions are based. The eleven variables include the three Demographic controls, six Parent Values (five separate Rural Values constructs plus overall Parent Religiosity), the mediating variable (Parent Involvement) and the dependent variable (Student GPA). The tables that follow present the regressions on the variables addressed in those Research Questions. 
Table 14

Pearson r Correlation Matrix of Variables Utilized in Multiple Regressions $(N=38)$

\begin{tabular}{|c|c|c|c|c|c|c|c|c|c|c|c|}
\hline & 1 & 2 & 3 & 4 & 5 & 6 & 7 & 8 & 9 & 10 & 11 \\
\hline 1 (SES) & -- & & & & & & & & & & \\
\hline $2(\mathrm{FS})$ & .012 & -- & & & & & & & & & \\
\hline 3 (SDI) & -.208 & .235 & -- & & & & & & & & \\
\hline $4 \mathrm{RV}-\mathrm{AI}^{\mathrm{a}}$ & -.019 & .189 & .126 & -- & & & & & & & \\
\hline $5 \mathrm{RV}-\mathrm{SC} / \mathrm{T}^{\mathrm{c}}$ & .257 & $.351 *$ & -.005 & .275 & -- & & & & & & \\
\hline $6 \mathrm{RV}-\mathrm{E}^{\mathrm{b}}$ & .311 & .148 & -.047 & -.006 & .151 & -- & & & & & \\
\hline $7 \mathrm{RV}-\mathrm{SE}^{\mathrm{a}}$ & .318 & .142 & -.170 & .018 & .299 & $.462 * *$ & - & & & & \\
\hline $8 \mathrm{RV}-\mathrm{GOB}^{\mathrm{c}}$ & -.152 & -.004 & $.326^{*}$ & $.421 * *$ & .101 & -.206 & .079 & - & & & \\
\hline $9 \mathrm{PR}^{\mathrm{c}}$ & $.415^{* *}$ & -.143 & $-.357^{*}$ & .155 & .001 & $.399 *$ & $.514^{* *}$ & -.014 & -- & & \\
\hline $10 \mathrm{PI}^{\mathrm{c}}$ & .297 & -.109 & -.089 & -.039 & .039 & $.364^{*}$ & $.464 * *$ & -.077 & $.502^{* *}$ & -- & \\
\hline $11 \mathrm{GPA}$ & .194 & -.155 & $-.573 * *$ & $-.349 *$ & -.084 & .213 & .159 & -.268 & .253 & .192 & -- \\
\hline
\end{tabular}


Table 14 (continued).

Note. $1=(\mathrm{SES}) ; 2=(\mathrm{FS}) ; 3=(\mathrm{SDI}) ; 4=\mathrm{RV}-\mathrm{AI} ; 5=\mathrm{RV}-\mathrm{SC} / \mathrm{T} ; 6=\mathrm{RV}-\mathrm{E} ; 7=\mathrm{RV}-\mathrm{SE} ; 8=\mathrm{RV}-\mathrm{GOB} ; 9=\mathrm{PR} ; 10=\mathrm{PI} ; 11=$ GPA.

${ }^{\mathrm{a}}$ Scale is based on Parent and Student Combined responses.

${ }^{\mathrm{b}}$ Scale is based on Parent only responses.

${ }^{c}$ Scale is based on Student only responses.

${ }^{*} p<.05 .{ }^{* *} p<.01$. 


\section{Research Question 1}

How do Demographic factors relate to Student Outcomes?

Table 15 presents the regression of Student Outcomes (measured by GPA) on Demographic controls: Socioeconomic Status (SES), Specially Designed Instruction (SDI), and Family Structure (FS). The stepwise regression is significant, $F(1,36)=6.89$, $p<.001$. SDI is negatively correlated; the effect size (Adjusted $R^{2}$ ) is .310 . In this equation, being in special education equates to a decrease in GPA of almost 14 points.

Table 15

Regression of GPA on Demographic Control Variables

\begin{tabular}{|c|c|c|c|c|c|}
\hline & Model 1 & & & & \\
\hline Multiple $R$ & .573 & & & & \\
\hline$R$ Square & .328 & & & & \\
\hline Adjusted $R$ Square & .310 & & & & \\
\hline Standard Error & 6.89 & & & & \\
\hline \multicolumn{6}{|c|}{ Analysis of Variance } \\
\hline & Sum of Squares & $d f$ & Mean Square & & \\
\hline Regression & 837.110 & 1 & 837.110 & & \\
\hline Residual & 1711.442 & 36 & 47.540 & & \\
\hline Total & 2548.553 & 37 & & & \\
\hline$F=17.609$ & \multicolumn{2}{|c|}{ Significant $F<.001$} & & & \\
\hline \multicolumn{6}{|c|}{ Variables in the Equation } \\
\hline Variable & $B$ & $S E B$ & Beta & $t$ & Sig $t$ \\
\hline Constant & 88.485 & 1.200 & & 73.722 & .000 \\
\hline SDI & -13.885 & 3.309 & -.573 & -4.196 & .000 \\
\hline
\end{tabular}

Note. $N=38$.

Research Question 2

How do Demographic factors relate to Parent Values: (a) Rural Values, and (b) 


\section{Parent Religiosity?}

Six separate regressions were required to answer this question. The results from the psychometric analyses above indicated that the five Rural Values scales could not be collapsed into a single Rural Values construct. Accordingly, each of the five Rural Values is regressed on the demographic factors separately. For this exploratory case study, with very small $N$, the decision was made not to do multivariate analysis with the five Rural Values as multiple dependent variables. The five Parent Religiosity scales did collapse to an overall Parent Religiosity (PR) construct, the criterion for the sixth regression.

Anti-Intellectualism (based on Combined responses) was the first Rural Value. The variables entered into the stepwise regression did not produce a significant model, indicating that none of the demographic factors had any influence on Anti-Intellectual values. Table 15 confirms the low correlations between Anti-Intellectualism and the Demographic factors.

For Social Conservatism/Traditionalism (Table 16), however, the regression was significant, $F(1,36)=5.070, p=.031$, with an effect size of .099 . Family Structure is the significant variable. The standardized Beta reflects that a one unit change in FS results in an increase in Social Conservatism/Traditionalism of just over a third of a standard deviation (.351). Of interest is the positive relationship; as the number of dependents increases, Social Conservatism/Traditionalist values get stronger. 
Table 16

Regression of Social Conservatism/Traditionalism on Demographic Control Variables

\begin{tabular}{|c|c|c|c|c|c|}
\hline & Model 1 & & & & \\
\hline Multiple $R$ & .351 & & & & \\
\hline$R$ Square & .123 & & & & \\
\hline Adjusted $R$ Square & .099 & & & & \\
\hline Standard Error & .69324 & & & & \\
\hline \multicolumn{6}{|c|}{ Analysis of Variance } \\
\hline & Sum of Squares & $d f$ & Mean Square & & \\
\hline Regression & 2.437 & 1 & 2.437 & & \\
\hline Residual & 17.301 & 36 & .481 & & \\
\hline Total & 19.738 & 37 & & & \\
\hline$F=5.070$ & \multicolumn{2}{|c|}{ Significant $F=.031$} & & & \\
\hline \multicolumn{6}{|c|}{ Variables in the Equation } \\
\hline Variable & $B$ & $S E B$ & Beta & $t$ & $\operatorname{Sig} t$ \\
\hline Constant & 2.127 & .310 & & 6.866 & .000 \\
\hline FS & .617 & .274 & .351 & 2.252 & .031 \\
\hline
\end{tabular}

Note. $N=38$; scale is based on Parent only responses.

The next Rural Value, Ethnocentricity, was regressed on Demographic Controls. As shown in Table 17, the stepwise regression was significant, $F(1,36)=4.059, p=.057$ with a minimal effect size of .076 . Socioeconomic Status (SES) was the significant variable, with a standardized beta of just under a third of standard deviation (.318). Again, the direction is worth noting; higher SES is associated with stronger Ethnocentric values. 
Table 17

Regression of Ethnocentricity on Demographic Control Variables

\begin{tabular}{|c|c|c|c|c|c|}
\hline & Model 1 & & & & \\
\hline Multiple $R$ & .311 & & & & \\
\hline$R$ Square & .097 & & & & \\
\hline Adjusted $R$ Square & .072 & & & & \\
\hline Standard Error & .58828 & & & & \\
\hline \multicolumn{6}{|c|}{ Analysis of Variance } \\
\hline & Sum of Squares & $d f$ & Mean Square & & \\
\hline Regression & 1.336 & 1 & .336 & & \\
\hline Residual & 12.459 & 36 & .346 & & \\
\hline Total & 13.795 & 37 & & & \\
\hline$F=3.860$ & Significant $F=.057$ & & & & \\
\hline \multicolumn{6}{|c|}{ Variables in the Equation } \\
\hline Variable & $B$ & $S E B$ & Beta & $t$ & $\operatorname{Sig} t$ \\
\hline Constant & 3.384 & .305 & & 11.104 & .001 \\
\hline SES & .260 & .133 & .311 & 1.965 & .057 \\
\hline
\end{tabular}

Note. $N=38$; scale is based on Parent only responses.

The stepwise regression for Stewardship of Earth represents the fourth Rural Values construct. Table 18 demonstrates a significant ANOVA, $F(1,36)=4.059, p=$ .051 , explaining $7.6 \%$ of the variance. The significant demographic variable is SES, again with a Beta of just under a third of a standard deviation (.318). 
Table 18

Regression of Stewardship of Earth on Demographic Control Variables

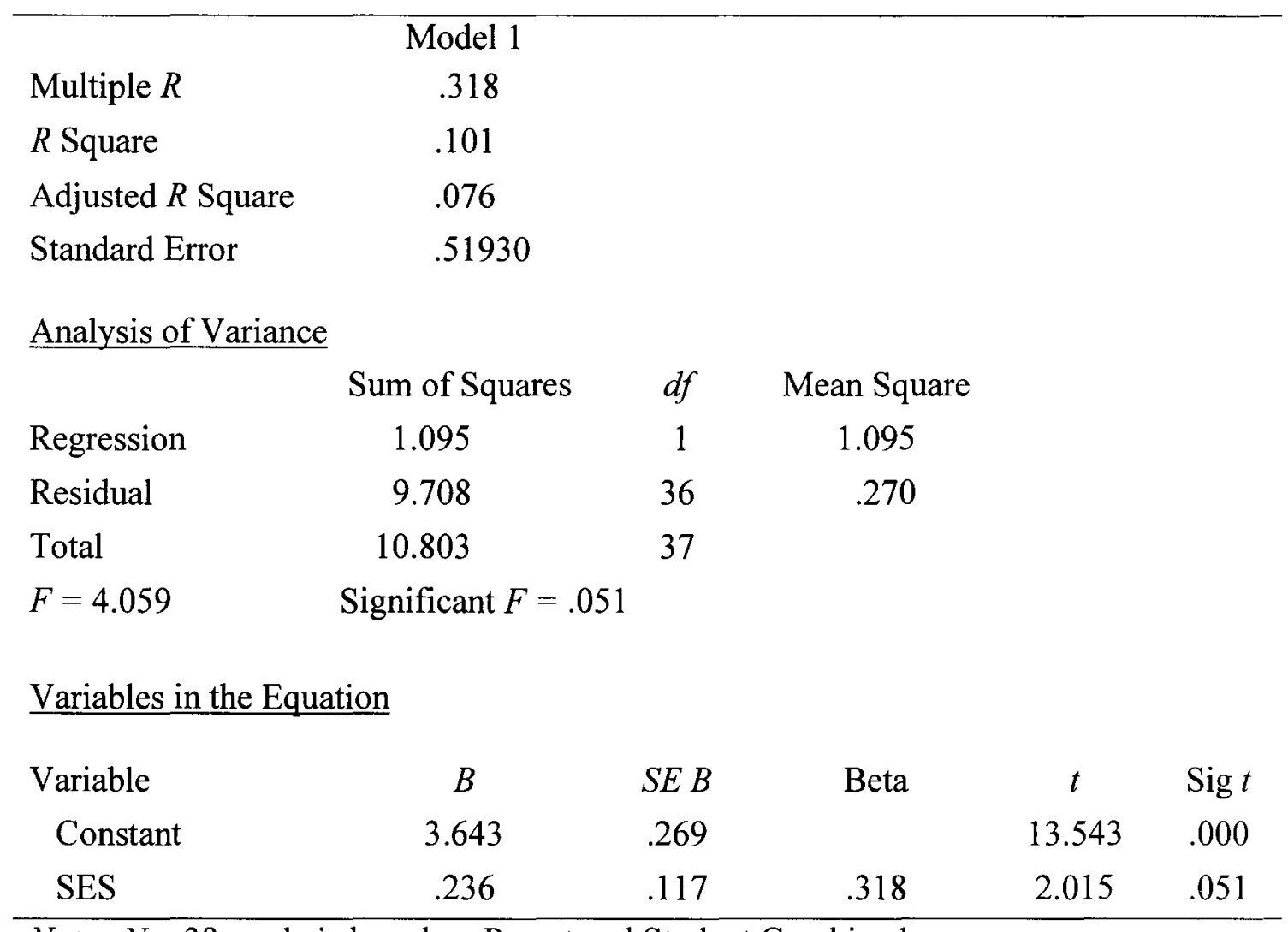

Note. $N=38$; scale is based on Parent and Student Combined responses.

The regression for the final Rural Values construct, Good Old Boy, is disclosed in Table 19. The equation indicates a significant relationship, $F(1,36)=4.274, p=.046$, with minimal effect size of .081. The significant variable is Specially Designed Instruction (SDI). The positive correlation indicates that having a child in special education is associated with stronger Good Old Boy values. 
Table 19

Regression of Good Old Boy on Demographic Control Variables

\begin{tabular}{|c|c|c|c|c|c|}
\hline & Model 1 & & & & \\
\hline Multiple $R$ & .326 & & & & \\
\hline$R$ Square & .106 & & & & \\
\hline Adjusted $R$ Square & .081 & & & & \\
\hline Standard Error & .89795 & & & & \\
\hline \multicolumn{6}{|c|}{ Analysis of Variance } \\
\hline & Sum of Squares & $d f$ & Mean Square & & \\
\hline Regression & 3.446 & 1 & 3.446 & & \\
\hline Residual & 29.027 & 36 & .806 & & \\
\hline Total & 32.474 & 37 & & & \\
\hline$F=4.274$ & Significant $F=.046$ & & & & \\
\hline \multicolumn{6}{|c|}{ Variables in the Equation } \\
\hline Variable & $B$ & $S E B$ & Beta & $t$ & $\operatorname{Sig} t$ \\
\hline Constant & 3.076 & .156 & & 19.677 & .000 \\
\hline SDI & .891 & .431 & .326 & 2.067 & .046 \\
\hline
\end{tabular}

Note. $N=38$; scale is based on Student only responses.

The second part of Research Question 2 deals with the regression of Parent Religiosity on Demographic Factors. Because the Parent Religiosity constructs were cohesive, they were treated as a composite variable. As can be observed in Table 20, the stepwise regression produced two significant models, with $F(1,36)=7.500, p=.010$ and $F(2,35)=5.804, p=.007$ for Models 1 and 2, respectively. Model 1 shows that SES has a significant relationship to Parent Religiosity with effect size of .149. In Model 2, SDI is added as a factor, but is negatively associated with PR. The effect size increases to a fifth of a standard deviation (Adjusted $R^{2}=.206$ ). The negative correlation for SDI means that families with a child in Specially Designed Instruction have lower Parent 
Religiosity.

Table 20

Regression of Parent Religiosity on Demographic Control Variables

\begin{tabular}{|c|c|c|c|c|c|}
\hline & Model 1 & Model 2 & & & \\
\hline Multiple $R$ & .415 & .499 & & & \\
\hline$R$ Square & .172 & .249 & & & \\
\hline Adjusted $R$ Square & .149 & .206 & & & \\
\hline Standard Error & .56351 & .54439 & & & \\
\hline \multicolumn{6}{|l|}{ Analysis of Variance } \\
\hline & Sum of Squares & $d f$ & Mean Square & & \\
\hline \multicolumn{6}{|l|}{ Model 1: SES } \\
\hline Regression & 2.382 & 1 & 2.382 & & \\
\hline Residual & 11.431 & 36 & .318 & & \\
\hline Total & 13.813 & 37 & & & \\
\hline$F=7.500$ & Significant $F=.010$ & & & & \\
\hline \multicolumn{6}{|l|}{ Model 2: SDI } \\
\hline Regression & 3.440 & 2 & 1.720 & & \\
\hline Residual & 10.373 & 35 & .296 & & \\
\hline Total & 13.813 & 37 & & & \\
\hline$F=5.804$ & Significant $F=.007$ & & & & \\
\hline \multicolumn{6}{|c|}{ Variables in the Equation } \\
\hline Variable & $B$ & $S E B$ & Beta & $t$ & Sig $t$ \\
\hline \multicolumn{6}{|l|}{ Model 1} \\
\hline Constant & 3.253 & .292 & & 11.142 & .000 \\
\hline SES & .348 & .127 & .415 & 2.739 & .010 \\
\hline \multicolumn{6}{|l|}{ Model 2} \\
\hline Constant & 3.426 & .297 & & 11.551 & .000 \\
\hline SES & .298 & .125 & .356 & 2.381 & .023 \\
\hline SDI & -.505 & .267 & -.283 & -1.890 & .067 \\
\hline
\end{tabular}




\section{Research Question 3}

How do Demographic factors relate to Parent Involvement?

The two Parent Involvement scales collapsed to an overall PI construct (see Psychometric Analysis of Scales, above). Concerning the influence of Demographic Factors on Parent Involvement, the stepwise regression (Table 21) showed a significant relationship, $F(1,36)=3.482, p=.070$. Only $6.31 \%$ of the variance on Parent Involvement is associated with SES, the significant demographic variable.

Table 21

Regression of Parent Involvement on Demographic Factors

\begin{tabular}{|c|c|c|c|c|c|}
\hline & Model 1 & & & & \\
\hline Multiple $R$ & .297 & & & & \\
\hline$R$ Square & .088 & & & & \\
\hline Adjusted $R$ Square & .063 & & & & \\
\hline Standard Error & .50652 & & & & \\
\hline \multicolumn{6}{|l|}{ Analysis of Variance } \\
\hline & Sum of Squares & $d f$ & Mean Square & & \\
\hline Regression & .893 & 1 & .893 & & \\
\hline Residual & 9.236 & 36 & .257 & & \\
\hline Total & 10.129 & 37 & & & \\
\hline$F=3.482$ & Significant $F=.070$ & & & & \\
\hline \multicolumn{6}{|c|}{ Variables in the Equation } \\
\hline Variable & $B$ & $S E B$ & Beta & $t$ & $\operatorname{Sig} t$ \\
\hline Constant & 2.893 & .262 & & 11.024 & .000 \\
\hline SES & .213 & .114 & .297 & 1.866 & .070 \\
\hline
\end{tabular}




\section{Research Question 4}

How do Parent Values (Rural Values and Parent Religiosity) relate to Student Outcomes?

For this question, the dependent variable is GPA. Based on the results of the psychometric work, there are six independent variables: the five separate Rural Values scales and overall Parent Religiosity. Table 22 presents the stepwise regression, which produces two significant models. In Step 1, Anti-Intellectualism (RV-AI) is negatively related to GPA, $F(1,36)=5.008, p=.032$, with Adjusted $R^{2}$ of .098 . In Step 2, Parent Religiosity is added to the equation, $F(2,35)=4.910, p=.013$, with the effect size increasing to .174 . Thus, for this regression, RV-AI has a negative effect on GPA while PR has a positive influence. 
Table 22

Regression of Student Outcomes on Parent Values (Rural Values and Parent

Religiosity)

\begin{tabular}{lcc}
\hline & Model 1 & Model 2 \\
Multiple $R$ & .349 & .468 \\
$R$ Square & .122 & .219 \\
Adjusted $R$ Square & .098 & .174 \\
Standard Error & 7.88335 & 7.54072
\end{tabular}

Analysis of Variance

Sum of Squares $\quad d f \quad$ Mean Square

Model 1: RV-AI

$\begin{array}{lrcr}\text { Regression } & 311.251 & 1 & 311.251 \\ \text { Residual } & 2237.302 & 36 & 62.147 \\ \text { Total } & 2548.553 & 37 & \end{array}$

$F=5.008 \quad$ Significant $F=.032$

Model 2: PR

$\begin{array}{lccc}\text { Regression } & 558.367 & 2 & 279.184 \\ \text { Residual } & 1990.185 & 35 & 56.862 \\ \text { Total } & 2548.553 & 37 & \end{array}$

$F=4.910 \quad$ Significant $F=.013$

Variables in the Equation

\begin{tabular}{lccccc} 
Variable & $B$ & $S E B$ & Beta & $t$ & Sig $t$ \\
Model 1 & & & & & \\
Constant & 100.115 & 6.148 & & 16.285 & $<.001$ \\
RV-AI & -4.794 & 2.142 & -.349 & -2.238 & .032 \\
Model 2 & & & & & \\
Constant & 84.823 & 9.401 & & 9.022 & $<.001$ \\
RV-AI & -5.466 & 2.074 & -.398 & -2.635 & .012 \\
PR & 4.282 & 2.054 & .315 & 2.085 & .044 \\
\hline
\end{tabular}

Note. $N=38$. 


\section{Research Question 5}

How do Parent Values (Rural Values and Parent Religiosity) relate to Parent Involvement?

For Research Question 5, the five Rural Values Scales and Parent Religiosity are the independent variables, with Parent Involvement as the dependent variable. Table 23 gives the results of the stepwise regression. Two models are produced. Parent Religiosity enters in Step 1, $F(1,36)=12.130, p=.001$, explaining $23 \%$ of the variance. In Step 2, Stewardship of Earth enters, $F(2,35)=7.846, p=.002$, with the effect size increasing to .27 . In this equation, both PR and RV-SE have a positive effect on Parent Involvement. 
Table 23

Regression of Parent Involvement on Parent Values (Rural Values and Parent

Religiosity)

\begin{tabular}{lcc}
\hline & Model 1 & Model 2 \\
Multiple $R$ & .502 & .556 \\
$R$ Square & .252 & .310 \\
Adjusted $R$ & .231 & .270 \\
Square & & \\
Standard Error & .45876 & .44702
\end{tabular}

Analysis of Variance

Sum of Squares $\quad d f \quad$ Mean Square

Model 1: PR

Regression

2.553

2.553

Residual

7.577

1

.210

Total

10.129

36

37

$F=12.130$

Significant $F=.001$

Model 2: RV-SE

Regression

3.136

2

1.568

Residual

6.994

35

.200

Total

10.129

37

$F=7.846$

Significant $F=.001$

Variables in the Equation

Variable

$$
B
$$

$S E B$

Beta

$t \quad \operatorname{Sig} t$

Model 1

Constant

PR

1.633

.430

Model 2

\begin{tabular}{lccccc} 
Constant & 1.001 & .612 & & 1.635 & .111 \\
PR & .307 & .140 & .358 & 2.188 & .035 \\
RV-SE & .271 & .159 & .280 & 1.708 & .097 \\
\hline
\end{tabular}

Note. $N=38$. 


\section{Research Question 6}

How does Parent Involvement relate to Student Outcomes?

This question investigates the influence of Parent Involvement on Student Outcomes (GPA). The stepwise regression produced no significant model. This confirms the low correlation between Parent Involvement and GPA in Table 14, $r=.192$.

\section{Research Question 7}

How do Parent Values (Rural Values and Parent Religiosity) relate to Student Outcomes, controlling for Demographic factors?

Beginning with Research Question 7, the multiple regressions utilized were hierarchical, with the order of variables based upon Figure 1, the theoretical model. Only the variables that demonstrated significance in Research Questions 1-6 were forwarded to these hierarchical analyses.

For Research Question 7, the results of Research Questions 1, 2, and 4 are relevant. In Research Question 1, Specially Designed Instruction had a significant effect on student GPA. For Research Question 4, two Parent Values had an influence on student GPA: Rural Values-Anti-Intellectualism and Parent Religiosity. Research Question 2 revealed that none of the three demographic factors was significantly associated with Anti-Intellectualism. However, both socioeconomic status and Specially Designed Instruction were significantly related to PR.

Table 24 presents the hierarchical regression for Research Question 7: two Demographic factors (SES and SDI) were entered first, as a control; then Rural ValuesAnti-Intellectualism and Parent Religiosity were entered, to examine their effect net of the control variables. The dependent variable is GPA. 
In Model 1 , SDI is significant, $F(1,36)=17.609, p<.001$, with an Adjusted $R^{2}$ of .310. Model 2 is also significant, $F(2,35)=11.992, p=<.001$, and the effect size increases to .373 as Anti-Intellectualism is added to the equation. Both variables are negatively correlated. Thus the majority of the effect comes from having a child in special education (lower grades), while Anti-Intellectualism further depresses grades after SDI has been controlled. Parent Religiosity drops out of the equation when the demographic factors are entered first. 
Table 24

Regression of Student Outcomes on Parent Values while Controlling for Demographic Factors

\begin{tabular}{lcc}
\hline & Model 1 & Model 2 \\
Multiple $R$ & .573 & .638 \\
$R$ Square & .328 & .407 \\
Adjusted $R$ Square & .310 & .373 \\
Standard Error & 6.89493 & 6.7319
\end{tabular}

Analysis of Variance

Sum of Squares $\quad d f \quad$ Mean Square

Model 1: SDI

$\begin{array}{lrcr}\text { Regression } & 837.110 & 1 & 837.110 \\ \text { Residual } & 1711.442 & 36 & 47.540 \\ \text { Total } & 2548.553 & 37 & \end{array}$

$F=17.609 \quad$ Significant $F<.001$

Model 2: RV-AI

$\begin{array}{lrrr}\text { Regression } & 1036.313 & 2 & 518.156 \\ \text { Residual } & 1512.240 & 35 & 43.207 \\ \text { Total } & 2548.553 & 37 & \end{array}$

$F=11.992 \quad$ Significant $F<.001$

Variables in the Equation

\begin{tabular}{lccccc} 
Variable & $B$ & $S E B$ & Beta & $t$ & Sig $t$ \\
Model 1 & & & & & \\
$\quad$ Constant & 88.485 & 1.200 & & 73.722 & .000 \\
SDI & -13.885 & 3.309 & -.573 & -4.196 & .010 \\
Model 2 & & & & & \\
Constant & 99.224 & 5.130 & & 19.340 & .000 \\
SDI & -13.026 & 3.180 & -.538 & -4.096 & .000 \\
RV-AI & -3.866 & 1.800 & -.282 & -2.147 & .039 \\
\hline
\end{tabular}

Note. $N=38$. 


\section{Research Question 8}

How does Parent Involvement relate to Student Outcomes, controlling for Demographic factors?

For this hierarchical regression, the results of Research Questions 1, 3, and 6 are relevant. In Research Question 1, only Specially Designed Instruction (SDI) had an effect on GPA. Research Question 6 revealed that Parent Involvement had no effect on GPA. Research Question 3 demonstrated that Socioeconomic Status (SES) was significantly related to Parent Involvement, but because Parent Involvement has no effect on GPA, this relationship is moot, vis-à-vis indirect effects on Student Outcomes.

Thus, the hierarchical regression for this question reduces to a single demographic factor, SDI, entered as a control. Parent Involvement is not added in a second step (Research Question 6, above). The result is identical to Table 15. SDI is statistically significant, explains $31 \%$ of the variance, and is equivalent to about a 14 point reduction in GPA. For more detail, see Research Question 1 (above).

\section{Research Question 9}

How do Parent Values (Rural Values and Parent Religiosity) relate to Student Outcomes, as mediated by Parent Involvement?

This hierarchical regression is based on the results of Research Questions 4, 5, and 6. Research Question 4 demonstrated that of the five Rural Values scales and the composite Parent Religiosity, only RV-Anti-Intellectualism and Parent Religiosity are significantly related to GPA. Research Question 6 showed that Parent Involvement is not related to GPA. For Research Question 5, RV-Stewardship of Earth and Parent Religiosity both influenced Parent Involvement; however, because Parent Involvement 
does not affect GPA, these two significant relationships have no indirect effect on GPA.

Therefore, for this hierarchical regression, RV-Anti-Intellectualism and Parent

Religiosity are entered first; no mediating variable is entered because of the nonsignificant results from Research Question 6. This equation therefore reduces to GPA being regressed on the two variables--RV-AI and PR. The result of this is identical to Table 22. Both variables are significant, with a total Adjusted $R^{2}$ of .174 . AntiIntellectualism has a negative effect on GPA while Parent Religiosity has a positive effect. For detail, see Research Question 4 (above).

\section{Research Question 10}

How do Parent Values (Rural Values and Parent Religiosity) relate to Student Outcomes, controlling for Demographic factors, as mediated by Parent Involvement?

This question is a comprehensive measurement of all overlapping relationships within the entire concept model (Figure 1): the Demographic controls, the Parent Values (five Rural Values and Parent Religiosity), Parent Involvement as a mediating variable, and Student Outcomes as the criterion. Hierarchical regression was required to assess these relationships. The results of Research Questions 7-9 are relevant. Those findings indicated that only Specially Designed Instruction (SDI) among the controls was related to achievement. Among the Parent Values, only RV-Intellectualism was significant when SDI was controlled. Parent Involvement was not significant. Thus, only two variables are entered into the equation. SDI is entered first, as a control. RV-AntiIntellectualism enters next, for its effect net of SDI. This is identical to the results of Research Question 7. Because Parent Involvement had no significant relationship to 
GPA, the mediating factor falls out of the full model (see Research Question 9, above).

Table 24, from Research Question 7, is identical to the full model. Thus for the entire set of variables in the theoretical model (Figure 1), only two are significantly related to student GPA: SDI and Anti-Intellectualism. The total variance explained is $37.3 \%$. Both factors are negatively related to achievement. For more detail, see Research Question 7, above.

\section{Qualitative Analysis}

Supplemental qualitative analysis is needed in a case study (Yin, 1989) because, as an intense investigation of one site, the case study depends on multiple perspectives for triangulation. The primary analysis of the 10 Research Questions for this study is limited by sample size and inability to test the model adequately with regression. In an exploratory case study, however, an investigation is meant to propose theory rather than to test theory. Using that as the foundation for analysis, three basic sources for uncovering pertinent supplemental information were utilized: Researcher Field Notes (RFN), interviews (staff, students, parents), and analysis of parent activity sheets, assessment reports, local newspapers, and other documents.

Throughout the duration of this study, extensive notations referred to as Researcher Field Notes (RFN) were made of elements reflecting Rural Values constructs as they were displayed. Those RFN provide details that shed light on the constructs under study. In addition, formal interviews were conducted with several student/parent pairs. Their comments were analyzed for pertinence to constructs and are reported as each construct is discussed. Because only the category of the parent/student pair is important to this study but not the names of the individuals, the interviewees are given 
titles to express their designated categories. If the parent-student pair category is relevant to the comments included below, their designations are provided: Low Involvement Parent $/$ Low Achievement Student = LL, Low Involvement Parent $/$ High Achievement Student $=$ LH, High Involvement Parent $/$ Low Achievement Student $=$ HL, High Involvement Parent/High Achievement Student $=\mathrm{HH}$, Medium Involvement Parent $/$ Low Achievement Student $=$ ML, Medium Involvement Parent $/$ High Achievement Student $=$ MH. Titles may be further designated by numbers to represent grades: for example, $7 \mathrm{HH}$ or $8 \mathrm{ML}$. In addition, the seventh grade $\mathrm{HH}$ parent also serves as a frequent substitute teacher at DEMS. She is named 7HHs. The $8 \mathrm{HHh}$ is a High Involvement Parent/High Achievement Student who withdrew to home school for the duration of the student's high school career. (See Appendix U for Interview Schedule.)

\section{Rural Values}

In addition to the setting of this study, further data collected provide for a richly textured description of Rural Values in the Dixon community. Researcher Field Notes (RFN), which include informal comments and other notations, formal interviews, and documents serve as resource for the thick description, the Qualitative Analysis, of the community under study. Anti-Intellectualism (RV-AI)

Few schools would admit to having something other than academics as their number one priority, but there are those who would suggest that the Anti-Intellectual trait does exist here. Consistent with the data reported in the Review of Literature, the rural Southerners in this school community voiced rejection of "book learnin" in preference to "good trainin' that can be used in ever'day life" (RFN). The custodian makes a truly 
astute observation. She says,

You can pretty much tell how supportive the community is of academics by the way kids treat school property. The walls here have shown a disrespect for the school that reflects their attitudes towards learning. Severe kick marks.

Thermostats torn off. Doors ripped off their hinges. Catsup on the ceiling. Toilet paper wads on the ceiling. Paper, towels, pencils, pop cans, plastic balls, and whole rolls of toilet paper thrown into the toilets. Sometimes "stuff" is taken out of the toilets and thrown around and smeared on the walls. They've even defecated in the floor.

Students are relatively candid about the value of academics in their school. $8 \mathrm{HHh}$ student, who was the class Salutatorian, admitted, "It certainly isn't an upbeat learning environment. There is no art program and no real music program. No elective options. And we weren't challenged." (8HHh student has since withdrawn to home school. By the time this study was conducted, she was a semester ahead of her peers and had expanded her transcript to include two years of Spanish, French, Irish, and Arabic, Equine Study, Calculus, and other varied units. Not a "geek" by any means, $8 \mathrm{HHh}$ student was a standout on the girls basketball team and competed on the cross-country and the swim team.)

7LL student believes, "The quality of education you receive at DEMS is not that good" while 8ML student classifies her education at DEMS as "so-so."

Echoing these sentiments, $8 \mathrm{MH}$ student draws some interesting conclusions:

You can tell that arts and humanities aren't valued here. Look at the stage. If you play basketball, you don't expect to have to share the gym with twenty other groups. But the stage, that is supposed to be used for drama or stage productions, is used for drill team and cheerleader practice, club meetings, dances, custodian storage, and some special ed classes. [In years past it was also used for wrestling practice and for storage of the huge, rolled up wrestling mats between practices.]

8HH student was slightly critical, saying, "I could have been more prepared [for high school]. I had to review a lot, especially math. We were weak a little there." 8LM 
student and 7LL student agreed, "Study hall was a waste. We never worked anyway."

Even the most positive of interviewees, 7HHs student, was also somewhat critical:

Popularity here is based on money, clothes, and ability in sports. Nobody really looks at grades. To most kids grades aren't important. Just pass, that's it. I think only about $50 \%$ of parents value good grades. Their priorities are $75 \%$ sports and $25 \%$ grades.

Most interviewed parents were also concerned about the lack of academic rigor. $8 \mathrm{HH}$ parent felt that her children had a good start for high school and postsecondary education at Dixon. "They were encouraged to achieve and keep striving and not give up." But $8 \mathrm{HH}$ parent added, "Sports is not the total emphasis here. But it is stressed. Things like foreign language are not valued." Commenting on the fact that Jr. Beta was discontinued at DEMS, 8HHh parent assessed the situation thusly, "I think the school administration and faculty demonstrated a lack of concern for academic clubs and academic competitions. Otherwise they wouldn't have dropped Beta."

7LM parent expressed concern for her son because, "My child did not play sports and, therefore, did not fit into a lot of crowds." But $8 \mathrm{MH}$ parent was resolute, "Sports is emphasized over everything else." 8HHh parent summarized with, "The school system sees sports as supremely important." 7MM parent said, "It was his [her son's] worst years of school." Another parent, 7LM, maintains, "Some students withdraw to go to the Christian school for a better education." $8 \mathrm{HHh}$ parent, who did withdraw her child from public school following her eighth grade at DEMS, agreed, "We left in order to have academic freedom to choose a wider variety of subjects, to be able to tailor the subjects to my child's interest, to meet her individual needs--which get overlooked in the public school system." Her comments were supported by $8 \mathrm{MH}$ parent: "Only core classes are 
important--no arts and humanities, no wide range of electives. I sometimes worry because they don't have any depth and the curricula are so limited." $8 \mathrm{HHh}$ parent finally issued this aggressive statement:

The board members are more concerned about missing light bulbs at the sports center than they are about curriculum. They don't understand education. One member is a mail carrier. One is a beautician. They have no sufficient background to be making decisions about our children's education. They don't have a clue of how to go about empowering education. The high school basketball team is the focal point of their existence.

Many staff members also believe that the sports program is paramount in the public school system. But no one would go on record to say as much. Only the custodian was willing to offer a comment: "Academics are not pushed here. The men run things and men like sports. They want athletes not scholars. Athletes can mean big bucks! Besides, arts and humanities is sissy stuff."

Artifacts found in the DEMS community suggest that the interviewees might be correct in their assessment of the situation. For instance, the Journal-Enterprise, the local community newspaper, is typically only twenty pages long. The February 6, 2003, issue covered the high school girls basketball games, the high school boys games, a Transylvania game, the neighboring school's games, county ABA (little league) games, a University of Louisville game, and the high school basketball homecoming. Also included were a story on a local rodeo champion and a "Down Memory Lane" basketball picture. One entire page was devoted to pictures of the Webster County Longspurs (turkey hunters) Banquet. Sports consumed six and a half pages of the recent issue, over $32 \%$ of the entire paper. Two small articles covered ACT exams and college aid forms, and a tiny article named a student making the dean's list at Eastern Kentucky University. One article, taking up less than a fourth of a page, detailed a school board meeting. 
Lunch menus were provided for several county schools. No other academic news was printed. (See a non-southern explanation for RV-AI, Appendix V.)

When teachers are hired to fill vacancies, it is well known that new faculty must agree to fill coaching positions before they are considered for a teaching assignment; regardless of the credentials they carry. The local radio station airs coverage of weekly basketball games. (The radio announcer covering the games is none other than the Assistant Superintendent who once played for Webster County and had her jersey retired into the school trophy case and is in the school Hall of Fame because of her basketball records.)

Sports offerings in the county system include basketball (boys, girls, freshmen, junior varsity, ABA), baseball (boys), softball (girls), soccer (girls), tennis (girls, boys, junior varsity), golf (girls, boys), track and field (girls, boys), and cross-country running (girls, boys, junior varsity). The administration has been known to dismiss school in order for students and personnel to travel with the basketball teams to state tournaments. Many classrooms forego regular studies to watch the NCAA basketball championship games and the World Series. Further evidence of sports priorities may be found in the school board policies concerning extracurricular activities. Athletic teams are furnished transportation to and from events. However, academic teams are required to have fundraisers in order to earn travel money. At the end of every season, sports banquets are held for each sport when members of the team, regardless of win or loss status, receive trophies. Only one academic award ceremony is held at the end of the year at the high school and at most of the county feeder schools. Moreover, with the Fall 2002 term, at the high school, Advance Placement classes were discontinued, no foreign language was 
offered, and the Art/Drama teacher was released and not replaced.

Two other examples of RV-AI might serve to make a point of priority. In late April 2002, the band director at the high school died suddenly. The school system issued no public recognition of his passing. No memorial was held in his honor even though he had been a faculty member of several years, serving as the treasurer of the Webster County Teachers Association for several of those years. In fact, few people other than band students and their parents were aware of his passing.

In contrast, the high school football coach also died suddenly, one month later in May 2002. Not only was there great public display of shock and dismay (in local newspapers, radio broadcasts, and regional television broadcasts), but also trauma specialists and grief counselors were called to the school the following day to comfort students and staff. In addition, the school community issued numerous pleas through local media to help erect a new football field house in his honor. The field house bears the name of the deceased football coach. (For insight into AI see Appendix W.)

While the preponderance of evidence indicates that academics are slighted in favor of support of sports, some parents and students were convinced that the system valued scores on achievement tests more than "real learning." For example, 7LL parent stated:

The main priority at that school is for the school to do well on the state test. They ran my child through, helped him on the state test by answering questions for him. They let him get by with little work--as if her could do no better. This caught up with him in high school. There were some good teachers, but many were not. They didn't care about the kids. They only wanted to teach the easy students. I don't feel the good teachers got support from the administration.

Understandably, 7LL student agreed, saying, "The most important thing at DEMS is CATS test scores." Other parents and students echoed these thoughts. "I believe the 
faculty is so driven by the school system to produce [on achievement tests], they don't have time to socialize," believes 8MH parent, "and curriculum at this school is based solely on CATS--means, ratings, rankings." Of course, $8 \mathrm{MH}$ student feels, "CATS is the big thing. All curricula are structured towards CATS." Even more emphatically, $8 \mathrm{HHh}$ parent says,

The public school system wants to herd the children in and out with homogenized, standardized academic results. The system is geared toward standardization which precludes having education in the truest sense. The students here are headed for hardships in trying to compete at the college level. This school doesn't have the caliber of teachers I wish my child to have. It doesn't have the funds necessary to have technology and projects that inspire the love of learning. It is very behind in science compared to other places. The students are there because they have to be. The love of learning is a scarce commodity.

$8 \mathrm{MH}$ parent has advanced degrees and is certified as a counselor in addition to

being a Baptist minister. He offers this observation:

I have always been education minded; I believe that education is the door to knowledge. It gives you power for many things. But if the parent doesn't value education, if it isn't modeled and motivated by parents, kids don't value it. A parent needs to be always seeking, trying to keep the education river flowing. The average parent here is not that motivated by educational opportunities. They are raised that way. They have their farms, their factories, or their county jobs. Education is not an important issue here. The goal for their kids is to get out of school, get a job, and make money. The ultimate irony here is that the individuals on the school board don't have the education to understand education methodology. It's absurd. It bothers me. The chair only has his high school diploma and he dictates policy for masters level teachers. How can an education system rise to a good level if their leaders are not educated?

Thus, whether intentional or unintentional, whether caused by the southern aptitude for underachievement or emphasis on sports or on standardized tests, whether controlled by the teachers, the parents, the principal, or the school board, there seem to be some definite clues to the Anti-Intellectualism in this school system. (One might also investigate the teachers' willingness to overlook male student absences during "deer 
season." Deer hunting is a significant rite of passage for young men, and some young hunters have boldly brought the results of their day in the woods to school, especially if their kill is particularly large or has a very large point count or spread.) Social Conservatism/Traditionalism ( $R V-S C / T)$

According to RFN, one characteristic of RV-SC/T is reflected at DEMS in a strong centralized leadership, embodied in a magisterial principal who lends a sense of stability to a chaotic process such as democratic decision-making. Evidence of RV-SC/T can be seen in the quasi-functioning Site Based Decision Making council (SBDM). Meant to serve as "self-governance" under Kentucky Education Reform Act (KERA), the DEMS SBDM council, under the current principal, gives the impression of little more than a rubber stamp for the principal's authoritative decisions. $8 \mathrm{HHh}$ parent reflected, "He doesn't allow the faculty to help run the school or to help determine the direction it needs to go. They can't exercise their expertise and do what they were trained and are paid to do." In order to maintain such tight control 8HHh parent believes, "He doesn't hire dynamic people to teach because they might threaten his authority." After his study of an issue at hand, the principal makes major decisions affecting hiring, budgeting, and other major governance of DEMS. He then presents his decisions to the SBDM at regularly scheduled meetings where the decisions undergo formal passage through a council vote. 7LL parent agrees that he is in control, "There is that site based decision thing, but I think the principal really makes the decisions."

However, tight control is viewed as a positive thing in a SC/T community. During formal interviews, $7 \mathrm{HHs}$ parent remarked that the principal could be commended because he was in "good control of every situation" and could be depended on to "take 
care of things as they come up." She also feels that the principal is a strong disciplinarian who makes the "kids toe the line and stay in line." Her child is safe, she said, with the principal in the building. He "goes by the book," she said. "I was pleased with his professionalism. He is a father figure. He requires a fearful respect. Kids know they need to obey." 7HHs student agrees with the parent. "He is hard and strict; everyone is intimidated by him. When he walks by, everybody gets quiet. He is definitely in control. I like that because there are less troublemakers and less harassment from kids."

Not all parents believe the principal's firm control of the environment to be completely positive, however. 8HH parent said, “Looking back, it wasn't such a good thing. He was overbearing and egotistical. And he set poor examples for our children. As it turns out, his morals were clouded." 7MM parent also expressed concern about the principal's questionable morals and his influence on the students at DEMS. The principal-centered conservatism was perceived by the custodian/parent as negative, but with qualification. She asserted, "The principal feels he is more important than anybody. Of course, he needs to be in charge to take care of problems."

Traditionalism also calls for a male-preferential organization. At DEMS, principal-selected "assistant principals" are male. During the 1999-2000 school, when the male principal was called out of the building, he appointed a male with no principal certification to fill in for him rather than appoint a female with principal certification, despite the fact that the ratio of male teachers to female was 4 to 18 . Moreover, when a training program for emerging new principals, during the 1998-1999 school year, was sponsored by the state board of education for Kentucky schools, the male principal of DEMS offered the opportunity to a male teacher who had sparse principal courses and no 
principal training or certification in his background. However, there were female teachers in the building with principal training and certification who wanted to be placed in the program offered by the state board.

Though no official protocol or designation established a department chair, a male teacher "assumed" the role and felt free to tell other teachers in the department what to do. He also made decisions that affected the entire department without checking with others first. Male teachers were given fewer "extra" duties and were chastised or disciplined less than women even if they behaved in the same way (e.g., arrived late, skipped faculty meetings, submitted incomplete forms). Male preference was also alluded to by the female custodian who was interviewed. She shared the story of how numerous times she had been treated prejudicially when passed over for special perks or assignments whiled her male cohort enjoyed not only fewer details, but also a friendly camaraderie with the principal. She felt she was unjustly criticized and accused of poor work. "He lets ___ [male custodian] off without saying anything to him. Even when he comes in late. And he talks down to me. Just because I'm a woman. He thinks he can get away with it. So far, he has."

Ethnocentricity $(R V-E)$

Identified as a culture's belief in itself as better than other cultures (Little, 1998), Ethnocentrism was revised to mean a community's belief in itself as better than other communities in Little and Miller (2001). Both facets of this Rural Value seem to be at work in the community under study. Community summer festivals take on an air of competition with each community vying for the most people in attendance and the largest gate proceeds. The elementary ABA basketball and football leagues at each school 
establish a competitive spirit between the county schools. Even after graduating into high school, the students carry their elementary/middle school identities with them, and the competitive spirit spills over into the high school setting. Sweatshirts, jackets, sweaters, and book bags bearing the feeder school names and logos are clearly visible at the high school. Some parents that were interviewed felt that Community-Centrism affected the way teachers treated their children. "The teachers didn't treat my child well because he came from another school. They thought he would be unruly and not up to standard," claimed 7LM parent. "Some kids got by with anything," concurred 7LL parent, suggesting that teachers were prejudiced against the others.

Despite these Community-Centric traditions, regional barriers seem to dissolve when their rural ethnicity is challenged by their urban neighbors. Very little of southwestern Kentucky can be called urban. In fact, the closest large city to Dixon is Evansville, Indiana. Though Evansville is fewer than fifty miles and less than an hour's drive away, it is on the north side of the Ohio River. The Yankees, Hoosiers, or city slickers, as they are called, are the objects of fierce rivalries and the cause of much heated resentment. Ridiculed when they travel across the river to work or ship, Dixonians often endure barbs and insults. Self-appointed northern sophisticates feel compelled to ask the "hillbillies and "rednecks" pejorative questions: Do you always go barefoot? Did you marry your cousin? How many years did it take for you to get out of the eighth grade? Does your family have a Bubba? Did your daddy take a shotgun to your wedding? Even Jay Leno sometimes wonders aloud these questions about Kentuckians on the Tonight Show.

Derogatory ethnic jokes are re-written to insult rural Kentuckians. "How many 
Kentuckians does it take to change a light bulb? Three. One to hold the bulb, two to turn the stool he's standing on." "The bridge was out over the Ohio. A Hoosier tried to convince a Kentuckian to walk across on a flashlight beam. The Kentuckian refused saying, 'Oh, no! I'm smarter than that. As soon as I get halfway across, you'll turn off the flashlight."” (See also Appendices V and W.)

Stewardship of Earth (RV-SE)

Perhaps one of the most difficult constructs to articulate is Stewardship of Earth. Though the concept of being responsible for protecting the land for future generations lies deep in the rural American heart, it is one of those tacit pieces of knowledge discussed earlier--one of the things people know but cannot say. Rural culture honors connection to the land and the males who maintain it even though men have been known to risk vast wealth, marriages, and families trying to keep the farm going, only to face bankruptcy. There seem to be no words that fully convey the intensity of emotion relative to a man's ownership of his land, but perhaps the phrase that most aptly expresses the sense of connection is "he has mud running through his veins."

As an illustration of this reality, many farms have been passed down through generation after generation. Giving birth to five children during the pre-World War II years, one Dixon couple worked hard to establish a large, profitable family farm. After the war, three of four sons returned from military service to live on and work for the family farm, eventually marrying and starting their own farms nearby. The one son who left moved to the city was ridiculed and deemed a "city slicker." The daughter stayed close to home to help care for her parents and farm with her husband after her marriage. Over the years, eight of the nine living grandchildren and seven of the great- 
grandchildren have returned to work and live on the farms. The original children are still living, so it is yet to be seen what will happen to the family farms at the end of this generation.

Farmers truly put down roots and farming becomes almost a spiritual endeavor. As a middle-aged farmer commented,

I feel I'm part of God's plan here. I, in a small way, help feed the world. Without me and people like me, we would all go hungry. I am expected to do my part, even if it's hard. The reward is not in the money we get--there's very little of that. The reward is in knowing we're helping to dare for God's creation. I can't do anything else. Besides, I love the feel and the smell of the dirt. I love seeing a field of nice straight rows. I love breathing fresh air and being out in the open so I can see nature all day long. (L. Tucker, personal communication, August 1999)

While there are female farmers, most farmers are male. For those males, it is doubtful that anyone can truly understand the intimate relationship that develops between a farmer and his land unless he, too, has experienced this passionate love affair with nature. To a farmer, the land takes on a life, a personality of her own (L. Tucker, personal communication, 1999). They speak of The Land tenderly and with great affection, protecting her and taking care of her every need. Farmers may spend countless hours, beginning before sunup and ending long after sundown, for season after season, year after year, wooing her into giving birth to good crops. Each spring, before the ground has completely thawed, even before the last frost of winter, farmers feel a restless sense of urgency to start the new crop season. From spring through fall, they spend countless hours tilling, seeding, weeding, tending, and harvesting. During the winter they pull maintenance on equipment, greasing joints, changing tires, replacing bearings, checking sprockets, repairing machinery, and hauling grain--only to begin all over again the next spring. Their tie to the land and her demands are so great, there is almost a 
symbiotic relationship that develops between them. They often pour thousands of dollars every year on The Land while their female partners may struggle single handedly to keep the household together, the bills paid, and the children in shoes. They are so tied to her that abandonment, even in the face of financial ruin, is not possible. If there is no descendant available and interested, the patriarch mourns because The Land cannot survive without someone to give her life.

On the other hand, farmers' wives often view "The Land" as a temptress, the one who gets special care and attention while they sit home alone. Comprehensibly, wives speak of themselves as "farm widows" during planting and harvesting seasons.

Elements of the community that support RV-SE as a construct are the agriculture classes and the Future Farmers of America organization at the high school and the farmer classes offered by the local Extension Office and farm supply companies. Agriculture classes and FFA competitions are popular at the high school, though there are no such classes at DEMS. However, the principal is a former agriculture teacher. $7 \mathrm{HHs}$ student believes every student should be required to "take ag. Classes. It has to do with food, trees, plants--everything."

Good Old Boy (RV-GOB)

Riding a bucking pony headlong into the side of a barn, playing a quick game of Throw the Knife before supper, seeing who can last the longest jumping out of the hay loft onto corncobs below, "dragging" a pickup at speeds up to $120 \mathrm{mph}$ down a straightaway on the wrong side of the highway, smearing his face with the blood of his first pointed buck as he nurses the shoulder bruised from the kick of the high powered rifle Dad gave him for his tenth birthday: rites of passage for the Good Old Boy in 
training accepted by the local community as part of life. Defining the Good Old Boy (GOB) as a construct, however, is another one of those tacit pieces of learning. Or it may be like the quark, a particle of matter so small it can be detected only through evidence of where it has been. A male may earn his GOB status by earning a series of "stripes" to become a full-fledged GOB: Learning the Language, Expressing Anti-Government Sentiments, Revering Maleness, Owning Guns and Hunting, Driving Pickup Trucks, and Dressing the Part.

Learning the language. Learning to "talk rite" and to appreciate the country music language can earn a man a few stripes--even if he is not even from the country. During the dawn/dusk pow-wows in Webster County, Kentucky, farmers gather to form a symphony of "blues talk" that might sound like the following (Brown \& Tucker, personal interview, August 1999):

Got too much rain or ain't got nuff rain. It's too hot. 'T's been too damn cold. Ground's too wet here, too dry there, too cloddy there, too fine here. Weeds'er takin' over. Beans 've got chemical damage.

Cain't get nuff hep. Nobody wants to work man'ule labor. Shit, too many of 'em triflin' or lazy.

Hell, cain't pay high nuff wages to compete with the fact'ries.

Guv'ment's all a bunch a crooks--not worried 'bout the farmer.

Cain't get no price for the crops. Hell, they's cheaper than when I got out of th' army in ' 45 .

Fert'lizer and seed's fifteen times higher.

They lettin' Argentina beans in--ship 'em plum over here 'cause they 'kin git 'em cheaper.

Dang EPA's so strict cain't really farm.

Warm winters make insects come worse. They eat the crops.

Haf t'pay prop'ty tax $n$ income tax $n$ godamned unmined coal tax.

'N everbody's wantin' sum'in fer nuthin.

Expressing anti-government sentiments. GOB stripes can be earned through expression of anti-government sentiments, which can be traced back to the days of the 
"Carpet Bagger" of Reconstruction, or to the "Revenuer" of Prohibition and the heyday of moonshine, or to the days of the Ku Klux Klan. It seems to the GOB mind that anytime he wants to "make money, have fun, or take care of his own," the "guv'ment's come a stickin' its goddam nose in!" In this community, it is well known that Segregationists still exist. It is known that there are pro-Ku-Klux-Klan feelings that ripple through the male population. It is also known that there are a few "night-rider" types still among us. But only those on the inside are privy to the identity of those involved. Openness would be too risky. But it goes without saying that association with the KKK would certainly hasten the speed at which a male might acquire his GOB status.

Of a less ominous nature are the moonshiners. More than one local ancestor made his fortune and amassed his farm acreage by the illegal production of corn liquor (white lightning or moonshine, so named because of its production and delivery by the light of the moon to make getting caught less likely). At one time, moonshine was said to be Kentucky's second largest cash export, just behind coal. Stills were located back woods so that "revenuers" could not find them. Young men earning their stripes were often posted at the entrance to ward off intruders and sound the alarm (gun shot) if need be. In addition to its illegal production, the delivery of moonshine was no less antigovernment. By the light of the full moon, apprentice GOBs in their souped-up cars or pickups, "ran" (delivered) the precious cargo (corn liquor). Status came quickly to the GOB who could out run the "cops," to the one who could manage the hair-raising back roads at ungodly speeds, and who could deliver the goods with not one bottle of home brew broken. Remnants of the moonshine runner survive today in the Saturday night drag racers and the legal driving age scoffed at by farmers teaching their boys to drive the 
hay truck as early as nine or ten years old.

Another form of anti-government interference can still be evidenced, though in not so blatant form. The anti-government "I'll-take-care-of-my-own" stripe can be probably recognized by the reader by merely mentioning the Hatfields and McCoys of Kentucky hill fame or the much maligned shotgun wedding. Though one would presume the "I'll-take-care-of-my-own" to be relegated to history, it is alive and well in this community. Officials know to "look the other way" or to ignore the GOB who is providing his own form of punishment to a "wayward child." While spouse abuse is not endorsed, it is not particularly frowned upon when used to keep a wife "in line." According to the GOB standard, "that's none of anybody else's business."

Small infractions of the law or small anti-government statements may take longer to establish GOB status, but they do count. Action such as hiding income with reports of grain loss, hunting out of season or without a license, owning unlicensed firearms, burning or dumping trash without a permit or on private property, abuse of set-aside farm programs, and use of sewer systems without septic tanks, all serve to earn GOB stripes. Even being especially vocal about unwanted government interference has merit, for instance, decrying the government protection of wetlands as 'the guv'ment takin' over my pop'ty so that some goddam duck hunter from the city can come down here and track up my fields!" A GOB act may be distinguished from an SOB (son-of-a-bitch) act because the GOB act is probably illegal but publicly sanctioned; the SOB act is probably legal but publicly unsanctioned. (See another example of anti-government sentiment in Appendix X.)

Revering maleness. Though mentioned briefly in this section, it might be 
necessary to say that in this patriarchy, the "boys" are valued more than the "girls." Historically, many farmers have considered it a failure to have only girl babies because "they couldn't work like boys and their jobs were to keep the house and the kids" (A. Brown, personal communication, 1999). Having boys adds stripes to a young man's GOB status; for, after all, the Good Old Boy is exactly that, a good old boy. Another of those tacit pieces of knowledge is that the GOB is a no-female club. The pre-dawn coffee klatches at mom-and-pop restaurants, the around-the-stove huddle at the hardware store, and the full bench in front of the courthouse are all void of female intrusion.

This male exclusivity owes its existence to the traditions common to most of rural America. But, in addition, it exists in part because of the strong male-dominant teachings of Southern religions. According to conservative denominations like the Southern Baptists, women are to be subservient to men (the Southern Baptists even include it as part of their mission statement). They are to hold no position of authority over males in church or Sunday School. In many places, they are prohibited from speaking from the pulpit and from praying and speaking aloud in the congregation. 7HHs student (Church of Christ member) commented on this practice: "Women can't pray aloud in church because we can't speak with authority over men at church or Sunday School. We aren't supposed to speak in the sanctuary during services."

The bus driver/pastor interviewed for this study arrived at the church (Southern Baptist) for his interview wearing jeans, cowboy boots, a suede leather Western style jacket, and a cowboy hat. (He is well known for his organization of annual trail rides. There are no areas with enough unfenced space locally, so he and twenty to thirty other riders load their horses into well-equipped stock trailers, then haul them forty miles to an 
area of free range near Kentucky Lake and spend several days riding and camping around campfires before circling back to the beginning.) During his interview, he quoted New Testament scripture to support the concept of female subservience.

A previous pastor search committee for his church questioned an applicant for the position of pastor, who was so certain of the Biblical mandates of female subjugation; he used his promotion of it as part of his credentials. According to his story, a church that had "called" (the meaning of call here is to hire) him earlier had dwindled down to only a few female members. Those female members decided he was the preacher their church needed, so they called him. Immediately after he became their preacher, he dispensed with all business meetings until the women (who had hired him) could compel their husbands, sons, brothers, or male acquaintances to attend so they could establish a male quorum to conduct business for the church. So convinced that women were to remain quiet in church (according to St. Paul), he silenced even the women who had called him and prevented them from performing any other church business. This high-schooleducated man boasted of this story as proof of his worthiness to other Southern Baptist churches looking for a pastor.

Displays of machismo are witnessed in games like Throw the Knife (see Graham, 2002) where two guys stand with their feet shoulder-width apart facing each other. They take turns throwing hunting knives at each other's feet to see who can get the closest without drawing blood. BB gun fights are one of the earliest exhibitions of a boy's male pride; more than one male currently wears a badly wounded eye like a badge of honor. Other feats of machismo include "layin' down rubber" at the center stoplight, dragging the nearest outhouse to the courthouse square and setting it on fire, driving a carload of 
drunk buddies through town so they can "moon" all the onlookers, surviving a night of snipe hunting, breaking a particularly devilish horse, "tipping" cows, "commando jumpin"' out of second-story hay lofts, and "belly-floppin' off a stripper pit." One fellow earned more than the regular share of GOB stripes after jumping off a stripper pit right into a nest of water moccasins. His points dramatically multiplied when he declined to have his snake-masticated penis lanced in the emergency room.

The principal of DEMS displays many of the attributes of a male-dominant culture. His leadership style is very dictatorial and he displays his preference for males in his failure to chastise them as often and as extensively for the same professional infractions (lateness, failure to complete reports, disregard for rules) that females commit and in his assignment of "perks" to male rather than female staff members. The custodian relates, "What he says goes. There's no interaction. He is a dictator, not open to criticism. But he's friendlier towards men. He's very condescending towards women in general."

Owning guns and hunting. Despite Biblical admonitions against violence, rural people take great pride in gun ownership and vehemently support the right to own guns. The reasons given for their valuing of guns are varied. According to the self-reporting of a seventy-eight-year-old, lifelong resident of Webster County, Kentucky, guns have been important for the "protection of crops against varmints and critters" and for the provision of "meat for the table" where oftentimes there would be none. Groundhogs, coyotes, and deer are well known for the damage they do to crops and livestock. And most farmers have many times supplied deer meat, rabbit meat, squirrel meat, quail meat, and 'coon meat for their family meals, especially in seasons of poor crops or prices. In addition, 
government intervention in most instances has hindered or handicapped the farmer through restrictions, taxes, regulations, or mandates, so gun control is viewed as just another threat or encroachment into the farmers' private lives (A. Brown, personal communication, August 1999).

Guns are everywhere: in the back window of pickups, in the front seat of cars, slung over shoulders, encased in special wooden cabinets with glass doors, hung on racks in the open on the living room walls, or above the mantle. It is not unusual for an eightyear-old to get his first rifle for a birthday present. Every male worthy of GOB stripes hunts. "Bragging rights" are granted to the GOB killing the biggest turkey, the biggest buck, the deer with the widest-spread or most-numerous-points rack, or the most quail/squirrel/rabbits/doves.

The February 6, 2003, issue of the local newspaper presents a full-page photojournal article called "Let's talk Turkey" (p. 3). It describes the gathering of 300 local residents at their "first annual" Longspurs meeting. This group of turkey hunters boasts 124 members; 12 are women but 62 are seventeen years old or younger. Of the seven pictures covering the entire page, three of them have close-ups of people younger than ten years old. One shows a nine-month-old "Little Gobbler" demonstrating the "turkey calling techniques she learned from her daddy." Another pictures two Longspurs "bagging" (like dead birds) two six-year-old boys. The third picture shoes a nine-yearold girl taking aim down the barrel of the Winchester 9410 rifle she has just won in the night's raffle.

During deer season, many hunters skip church or work to deer hunt. Coal miners are known to schedule their yearly vacation time around deer season. Driving through 
town during season, one will see pickup trucks, parked outside local restaurants or alongside the hardware store, with massive deer carcasses draining pools of blood into the truck bed and onto the pavement. Conversations inside the restaurants and hardware store focus on who killed what, where, and with what. Now and then, there is discussion about the one that got away. Newspapers are filled with "fresh kill" pictures. The younger the hunter, the bigger the rack, the better and bigger the picture is. Taxidermy is big business here. Deer head mountings, full turkey mountings, and other proof-of-thekill trophies are displayed alongside the honorable weapons above the fireplace or in the family room. (A non-hunter is considered to be less than masculine and merits, along with zero GOB stripes, monikers such as sissy, pansy, wuss, or--the worst--homo.)

Driving a pickup truck. Of course, the GOB usually drives a pickup truck; neither his work nor play would allow much else. In fact, the competitive spirit becomes aroused when the pickup is involved; it has to meet standards, too, unless it is the embodiment of the antithesis of a GOB Cadillac--a venerated antique that proudly sports its battle scars and, none the worse for them, keeps on running. A four-wheel-drive is all but required; a diesel is one level up. Wide tires, a big motor, and special-order bumpers are plusses. Adding to the stature are additions such as lift kits, a towing hitch, a fifth wheel, monster toolboxes, and of course, the gun rack in the window. Not infrequently, in the bed of the pickup is another indispensable tool of the trade: a good hunting dog. It does not matter what the breed of the dog, necessarily, as long as he can find, flush, and fetch.

Looking the part. One would not expect to see church-going attire on gun-toting males, and, indeed, that is not the typical picture. A GOB has his standards: jeans (preferably worn and faded), flannel shirt, baseball cap, and boots. Sometimes a hunting 
vest or coat adds a finishing touch.

The DEMS principal appears to be an example of a Good Old Boy. He wears cowboy boots, shirts, and hats to school. He foregoes formal ties for bolos with western insignia emblazoned on the front. He usually drives a well-kept pickup truck, but he has been known to ride one of his horses to school on a day when school pictures were to be made. He speaks with a long accentuated drawl and sprinkles "Yes, Ma'am's" and "Yes, Sir's" throughout a typical conversation. In addition to his job as principal at DEMS, he raises tobacco and chrysanthemums on his family farm. In his leisure time, he trains, shows, and sells bird dogs from his kennel all over the United States. His dog and gun collections are extensive. He hunts quail during season and, at times, schedules his vacations around hunting season so that he can take advantage of a large covey of quail.

Using the GOB system. One question remains: After earning his stripes, what special perks are bequeathed a GOB of stature? A male earns his GOB status, gaining his stripes through his anti-government stances, pickup, hunting prowess, country-talk/music culture, dress, and demonstrations of machismo. However, the main point of becoming a GOB is the power that the position can wield. There are doubters that such power exists, but rural Southerners around here tacitly accept it. 7LM parent agrees, "If you have money and parents with a name, you're treated well and expected to achieve [at school]." 7MM parent complained, "Kids that were doing the same thing he [her son] was doing [at school] got in no trouble whatsoever. Eventually my son was suspended from school for three days for a comment that was taken out of context." She continued, "Students are treated based on who they are. If the principal can get things accomplished through a student he or she is treated very well." 7LL parent is more explicit: "In my opinion the 
Webster County School Board and DEMS is a good-ole-boy system. They hire teachers who are friends and family even if they have no skills." $8 \mathrm{HHh}$ parent feels that "everybody knows everybody. They sit at the hardware store or on the courthouse bench or at the Lions Club and talk about everybody. A sense of community is really only alive at the high school gym."

The custodian remarked, "You can't trust the people you work with. They'll tell lies on you. Just to make themselves look better. It's all politics. Who you know and what you can do for them." $8 \mathrm{MH}$ parent adds, "It's just politics and power here. They aren't motivated by education. I'm not on the inside so my hands are tied by the local 'authorities.' The board chairman is just a Good Old Boy!'

A local example of "inbred, good-ol'-boy nepotism" may suffice to illustrate the true power of GOB in the community under study. A man, well into his eighties, is one of the best-known Good Old Boys in the county. He farms over 2,000 acres, drives several different pickup trucks, owns a small arsenal of weapons, and has displayed frequent and extreme examples of machismo with knives, guns, and fists. His hunting feats are legendary and his name is almost instantly recognizable. Over the past five years, he was reported four times by home health aides for abuse (of a spouse whose capacities had been diminished by senility). At one time the victim was taken to the doctor with bruises from head to toe. Social Services, city police, and the Sheriff's office were called in to witness the damage. Complaints were written up and the man was brought to the doctor's office. After several minutes, the victim was sent home--with the man. The charges against him sit on a desk, somewhere in the courthouse, not filed, despite the fact that even more instances have been reported. This man is a GOB. 


\section{Parent Religiosity}

The setting for this study provides ample evidence of the prominence religion garners in the tiny Dixon community; concentrated within less than five square miles, more than 30 churches of varied denominations hold weekly services. In addition, the eminent role of religion is demonstrated (RFN) by proclamations (Belief or PR-BEL) of community religiosity in road signs, bill boards, and newspaper articles; by community leaders who also serve as church leaders; by prayers offered at public functions; by religious services and activities provided as community entertainment (PR-RIT); by religious belief, religious action, spiritual experience (PR-EXP), primary affiliations (PRPAFF), and affiliation bond (PR-ABOND); by casual conversation (comments) and formal discussions (interviews); and in "news" articles, postings and advertisements in weekly community newspapers (documents). Belief (PR-BEL).

The interweaving of public life and religiosity is evident in documents readily found in the community. A half-page advertisement in the Journal-Enterprise, the oldest area weekly newspaper, professes, "The following businesses urge you to attend the church of your choice." The remainder of the space is filled with names of numerous businesses that pronounce their belief in God and their support of area churches. The advertisement closes with, "See you in church Sunday." Almost one-fourth of a page of the newspaper each week is dedicated to a feature article written by a local pastor. The article is usually based on Bible scripture and provides guidance in applying the biblical passage to daily life. Of the eight items posted on the weekly Bulletin Board ("Bulletin Board," 2003, p. 2), two were school related and two were religious in nature: the 
"Men's Prayer Breakfast" and "Diamond Baptist Church Youth Night."

Proclamations. At the entrance to Dixon city limits and most other county communities, along the major thoroughfares, the local ministerial associations place signs that welcome travelers. At the Dixon city limits the signs say, "The churches of Dixon welcome you. The Dixon Ministerial Association." Billboards in the area announce religious beliefs in three-foot letters: "Jesus is love" and "Jesus is the reason." In addition, the weekly newspaper frequently features a half-page advertisement sponsored by community businesses and individuals encouraging people, "Attend the church of your choice this Sunday." A summary of comments heard informally in and around the community provides a snapshot of the religious tone that permeates this small rural society. Comments attesting to the pervasiveness of religion into many facets of community life can be heard in the grocery store, in the doctor's office, in the post office, at the court house, at the funeral home, in The Trojan Inn, in OT's Restaurant, at the Federated Woman's Club, at the Webster County Retired Teachers Association meeting, at the Rose of Sharon Bible Study, and among spectators at children's dance lessons, ball games, track meets, tennis matches, and golf tournaments.

Ritual (PR-RIT)

"Blue laws" that once closed businesses on Sunday are pretty much relics of yesteryear; however, in Dixon no businesses are open on Sunday except for the grocery store between 12:00 noon and 5:00 PM. These and other actions in the community reflect the solid commitment to a religious stance.

Community leaders. The County Judge, whose two children were students of DEMS, is a leader in the General Baptist Church. He can often be seen presiding at 
community functions, such as the Cemmunity Easter Service or Community

Thanksgiving Service, leading an opening prayer. The Circuit Judge is a long-time member of the Baptist Church. She and her family regularly attend services near Dixon; her children attended the Christian Heritage Academy until its recent move; at the time data were collected for this study, they attended Dixon School. One bank president is General Baptist, one is Southern Baptist, and another is Methodist. The Superintendent of Schools during the first year of this study is a deacon in the General Baptist Church. The DEMS principal and his wife are active leaders in the Methodist Church; his daughters are leaders of the church y $y$ th groups. The teachers at DEMS are strongly vocal representatives of their Christian faith and churches: the science teacher, math teacher, and language arts teacher espouse the Baptist faith, and the social studies teacher is Church of Christ.

Public prayer. As a matter of official agenda, Webster County School Board meetings are opened with prayer. School graduations are opened and closed with prayer. Many school organizations open meetings with prayer led by an official chaplain. Women's clubs and men's clubs offer prayer as the first item of business. The Webster County Retired Teachers include a devotions leader as one of their Constitutional officers and open every meeting with a prayer. Coaches are often seen leading their teams in prayer before football, basketball, baseball, and golf games and before tennis matches and track meets. Parent Teacher Organization, Site Based Decision Making Council, Boy Scout, Girl Scout, 4-H, and Jr. Beta meetings are opened with Christian prayer. Community entertainment. The Assistant Superintendent of Webster County Schools organized and facilitated an annual event called Crusade for Christ for many 
years. She posted advertisements and sponsored promotions to herald the event in each elementary school. Later, she led the large gathering held in the high school gymnasium. The Crusade featured area Christian musical talent performances to "reach out and touch" the audience members with crowd-enlivening, hand-clapping, hallelujah-shouting gospel numbers, then emotion-laden, heart-wrenching, guilt-producing Christ-from-the-cross numbers. Local leaders offered their personal "testimonies" to enrapture the almost frenetic assemblage, then beseeched the throngs of youth and adults to "get right with God" or "give their lives to Christ." Hundreds and hundreds of participants answered these "altar calls" each year. [The Assistant Superintendent, the daughter of a wellknown General Baptist minister, was, at one time, a member of a gospel trio (The Vocalystics) that produced a commercially available gospel tape.]

Every small community within Webster County holds some sort of summer festival. For example, Dixon has the Freedom Festival, Providence has the Coal Festival, Wheatcroft has the Homecoming, Clay has Clay Days, Onton has Hay Days, Poole has Haze Daze, and Sebree has the Chicken Festival. During these annual events, the community activities almost always feature community prayer services and gospel singings.

Gospel singings are also held as fund-raising events to assist people who have incurred sickness, financial hardship, or the wrath of nature. Most recently, gospel singings produced thousands of dollars to help tornado victims in the Providence area. Also, in the past year, a cancer patient in Clay and a young leukemia patient in Sebree were provided funds through the efforts of area gospel groups who performed, then donated the proceeds from their events. 
Church attendance. According to local wisdom, one should belong to the Christian faith and should attend church whenever possible (or at least on Easter, Mother's Day, Thanksgiving week, and Christmas). People in authoritative positions (teacher, police, doctor, principal) should demonstrate that he or she is of the Christian faith and has a "home church." A good politician will believe in God, belong to a church, and behave according to "good" moral standards (or at least be discrete in misbehavior).

Pious poverty. Although the love of money is considered to be the root of evil, anyone who is lazy and not gainfully employed is almost as evil. On the other hand, a preacher should be "respectfully poor" and should not be wealthier than most of his congregation. Historically conservative rural preachers and their families have been expected to reflect a "pious poverty," never presenting an image of having, nor pursuing, wealth. Doing so would be classified as "uppity" or "vulgar"--even sinful. This stance seems to be a response, even if inappropriate, to scripture that instructs one to be humble. Many devout congregants have interpreted "humble" to mean "poor." Therefore, the most pious (the preacher) has been expected to be poor in order to maintain his humility.

Prayer and Bible reading. On the DEMS playground, students speak openly about religious beliefs. They conduct "prayer circles" at break and participated in "Meet You at the Flag Pole" prayer ceremonies. They carry Bibles with them to classes and to lunch. During the hour devoted weekly to Silent Sustained Reading in the social studies classroom, some students read from the Bible.

Public service. In this conimunity, Rural Values overlap Parent Religiosity and members are expected to help neighbors regardless of religious beliefs. Helping tornado victims during the summer of 2002 to find shelter, food, and clothing was a cause 
embraced by hundreds. Contributing to funds set up for cancer patients is a commonly supported activity. Area churches form teams to participate in the local Race for the Cure. Preachers assemble monthly to assess needs within community. As the Dixon Ministerial Association, they donate time and money to assist in situations deemed worthy of their attention. For example, they prepare baskets of food that are presented to indigent families each Thanksgiving. Congregations often donate food, clothing, and gifts to the local Baptist Temporary Shelter for Children.

As interpreted by believers of this community, "doing good" is considered Ritual: the action to support one's statement of belief and an indication of being a "real" Christian. From pulpits as well as from local political leaders, an underlying tenant of the Christian faith is often espoused: "Faith without works is dead."

Public school. Christianity is without doubt integrated into this public school. Specifically, certain areas emerged from formal interviews with parents, students, and staff as areas of special concerns: beliefs about evolution and the Ten Commandments (PR-BEL), school prayer (PR-RIT), the overlapping of the school and religion spheres of interest and activity (PR-ABOND), and the connection of religion (PR-PAFF) to student achievement (S0). (See Appendix X.)

The concept of evolution is not popular among DEMS parents, and teaching about evolution as accepted theory in the classroom is even less popular. $8 \mathrm{MH}$ and $8 \mathrm{HH}$ students believed that teachers taught the theory only because they had to. $8 \mathrm{HH}$ said, “Every teacher I had at DEMS pretty much told us they didn't believe in evolution. But they had to teach it. They avoided the teaching of creationism, though."

7HHs parent wanted to restrict the teaching of evolution further: "Teachers 
should not teach evolution. The Bible has been proven over and over countless times.

Evolution has no facts to stand on. Teaching evolution is an infringement on my child's rights. At the middle school age, kids are too young to have that crammed into them. The "big bang" is not true and should never be taught. Let evolution be talked about at home."

Interviewees were in general agreement on the posting of the Ten Commandments because they saw them as good guidelines for living, regardless of their religious nature. In the words of the custodian, "They are good rules for life. They fit in their own way into every religion." Home School parent added specifically, "There are similar guidelines in the Koran and cther religious documents."

Surprisingly, 7HHs offered a different position, but for an interesting reason. She said, "There is no reason for the Ten Commandments to be posted in school. They are moral principles. But they were written under the Mosaic Law. Christ changed everything." Her daughter more fully explained:

The Ten Commandments are in the Old Testament. We believe that the Old Testament is for learning. The New Testament, when Christ came, tells us what we are supposed to do now: hear, believe, repent, confess, be baptized. That's the gospel. The good news. You are supposed to repent of your sins, confess what you know and believe that Christ is the Son of God. Then you are to be baptized--fully immersed. The water isn't holy. But your belief and faith in God allows the water to wash your sins away.

School prayer was favored by most of the interviewees because, as Home School parent says, "I believe in prayer." More emphatically, 7HHs parent says, "I think God should be in the school. And if the teacher or student wants to lead a prayer, then that's OK." The students openly prayed at school according to 8LM student: "When somebody got hurt, we all got together and prayed." 
8MH parent, who moved here just before his child's eighth grade year, has a postgraduate degree and is a General Baptist preacher. He feels there is no conflict between his religious beliefs and DEMS activities. In fact, he was highly appreciative of them: "There were two prayers at DEMS graduation. The valedictorian and the salutatorian spoke openly about their Christian faith. I was impressed when I came from Princeton [Indiana]. Anything religious there had to be moved off campus. There was no prayer on campus. Here every ceremony has an opening and a closing prayer."

In spite of her professed strongly conservative views, $7 \mathrm{HHs}$ student expressed a dislike for "forced" prayers led by a teacher or over the intercom by the principal "because of the different religions among the students." The custodian agrees with her, stating, "Children have different beliefs; they should have a choice about prayers. I'm not opposed to a moment of silence. But a kid shouldn't have to listen to someone else's prayers."

Experience (PR-EXP)

Though only a prelude to the religious Primary Affiliation (PR-PAFF), the Experience (PR-EXP) realm of religion is so personal, so private, so intimate, that a communing with God is not something spoken of easily. But it is included here briefly because of the intense emotion, loyalty, and conviction it evokes in the religiously conservative Christian believer. PR-EXP is very real phenomena for the believer, and, as such, it cannot be denied. Consequently, it cannot be left out of the discussion on a respondent's Primary Affiliation. 8MH student says, "God is very real. If you know Christ, your life will change. I have always had a sense of a higher power." $8 \mathrm{HH}$ student feels that life is a challenge that he probably would not be able to handle without his faith 
in Jesus Christ. 7HHs parent believes, "There is an in-born need for a higher being. God is behind all ethics and morals."

Primary Affiliation (PR-PAFF)

The intensity of experience and belief sets the stage for life that is completely intertwined with religion. That intertwining is responsible for the high level of religious Primary Affiliation (PR-PAFF). Though DEMS is a public school, one would waste little time before encountering religiosity. 7LL parent notes, "The principal calls the boys, Brother--it was part of his religion." 8MH student believes that religion is such a pervasive element at DEMS because "this is the Bible Belt. Religion is a part of everybody's life." She illustrates, "My friends, my church, other youth--everybody here is accepting of the Baptist beliefs. There isn't any pressure towards religion, but there's a positive attitude towards Christianity." Because of her affiliations with church, she adjusted easily to her move to this area as an eighth grader. "When I moved here I didn't know anybody. But because of the kids in the youth group at my dad's church, I fit in pretty quickly and easily," she explains.

Affiliation Bond (PR-ABOND)

According to most of the parents and students interviewed for this study, Christianity is an accepted, perhaps an expected, element of daily public school life. Within the school day, praying, Bible reading, and discussing religious beliefs are entirely appropriate activities. Not only is religiosity a part of daily life, it is also, in some cases, the primary part. Evidence of PR-BOND is revealed in comments of respondents at DEMS. 7HHs student is a good example:

Most of my friends are at church. We socialize, eat together, go shopping, visit places like the Amish, and have fellowship time. Sometimes we have a Ladies 
Day Out. I don't go to many parties. Most parties are just dances. I go to the Church of Christ. We look at dancing as lust. It's like watching sexual moves on the dance floor.

8HH student concurs, "My five best friends all go to church with me." These students are two of the most popular in their classes. They are involved in numerous clubs and many sports, but church is their primary connection.

\section{Parent Involvement}

Contrary to statements made by the teachers and the principal concerning DEMS's openness to Parental Involvement (RFN), Parent Involvement Beliefs (PI-BEL) or sentiments expressed by the bus driver, the custodian, and most of the parents and students during interviews indicate that in reality, Parent Involvement Action (PI-ACT) at DEMS is neither broad nor deep. According to the subjects, the principal and the staff, through a variety of unspoken signals, discouraged active Parent Involvement and made the atmosphere "cold" and uninviting. Beliefs (PI-BEL)

During casual encounters with staff and parents, beliefs about parent involvement at DEMS were positive (RFN). Staff readily supported passive parent involvement-helping the child with homework and supplying extra resources, for example. Comments such as, "We know the more involved the parent is, the more successful the student will be," or "The best kids are the ones whose parents are involved," were offered by the Social Studies teacher. The Math Teacher suggested that, "The reason X gets into so much trouble is that he has no support at home."

Generally, parents are deemed welcome to participate in field trips, the fall festival, resource room activities such as stapling papers, and the annual Christmas party 
and Spring Dance. Notes taken at teacher's meetings also reflect positive attitudes toward active parent involvement. "I wish X's parents would get involved." "If X's parents ever showed up at a conference, I think we'd see a great improvement in his behavior." "Have you ever noticed that the kids who do well and give us no problems are the ones whose parents are the ones who come to the conferences?" "There's really no point in these conferences; we never get to talk to the parents that we need to see." "You can tell why $\mathrm{X}$ does so well; her parents are always up here."

The principal announced an "Open Door Policy" at the beginning Parent Teacher Conference. He stated, "Every parent is welcome in this school. We want you to be involved. We believe that students will succeed at a higher level if parents are involved in their education." Parents were admonished to, "First register in the office as a visitor," but they were encouraged to visit "at any time."

Some parents also expressed a belief in involvement as a way to promote their child's achievement in school: "If I keep after X, he does better." "X loves to have me up here." "I know that it is my job to help her with her work." "Teachers need all the support they can get."

Action (PI-ACT)

A dichotomy between belief and action seems apparent, however. Even though parents were expected to monitor homework, and most parents expressed their willingness to do so, comments made by many interviewees about the school atmosphere seemed resolute. The bus driver said, "I was ignored when I came to school." Parents from all categories offered, "The principal made it known that I shouldn't be there." "I could tell the teachers didn't want me around." "The atmosphere is very cold and 
uninviting when you enter the building." "Parent Involvement? It is not worth wasting my time." "The atmosphere seemed snooty. I felt the school would rather not have parents involved." From the custodian/parent: "There was very little encouragement for me to become involved. In fact, teachers were dismissive. Their body language said, 'Go away!'”

One parent, 8HHh, had withdrawn her children from Dixon to home school due, in part, to the lack of PI acceptance. She articulated the paradox surrounding PI that exists at DEMS: "Despite saying there is an open door policy, PI is treated suspiciously. You feel like you are being watched; you are not welcomed. The principal often interferes with PI."

Furthermore, some parents expressed a sense of distrust and alienation. "I was treated in a very unprofessional manner. I was lied to. And my son was a target. According to the principal my son could not even pee correctly. A student's word is worth absolutely nothing," lamented 7MM parent. Another parent, 7LL, said, "I felt manipulated by the system. I felt misled." $8 \mathrm{HHh}$ parent added, "Going to the school is like being a rat in a maze." The custodian echoed this sentiment, "The principal is a dictator, not open to criticism. He acts like he has a corncob up his butt. He made me feel worthless."

A highly educated parent, $8 \mathrm{MH}$, believed the unwelcoming atmosphere was generated in part by teachers' seemingly apparent dissatisfaction with working at DEMS. He said, "The atmosphere was not open or cordial and the staff was unfriendly. They were not very happy; there were no smiles or casual conversation. They certainly didn't seem like they were having a good time. I think they are teaching because it's a JOB, a 
paycheck, nothing more. If the staff is not happy, they are not going to be friendly to students or parents."

Even communication with parents was rated poor by most interviewees.

"Communication needs to be increased; it is a major problem," commented 8HH parent. "The principal has an inability to communicate with the staff and the community," agreed 7LM parent. "The only time I ever heard from the principal was when there was a problem, never about anything good," was offered by 7MM. 8HH said, "The principal was overbearing and egotistical."

Despite these disparaging remarks, parents who were known by the principal and staff felt they were welcomed when they visited the school. One parent/student pair, 7HHs, was outwardly supportive of the faculty and principal. They found the school to be open and the staff to be friendly and receptive: "The principal is a good person. And the staff is always nice and helpful." (It must be noted that this parent was a substitute teacher who frequently worked within the building and who was viewed as extremely important to the principal and faculty. This parent made other comments pertaining to Social Conservatism/Traditionalism that revealed her support of authoritative leadership.) One other parent (7ML), who was a frequent parent volunteer for school field trips, commented, "The staff was cordial," and "I felt welcomed to get involved." $8 \mathrm{HH}$ parent said, "The faculty was fine."

As a matter of official record, data concerning PI-ACT were revealed in the School Report Card (Dixon, 2000). Data provided in the School Report Card is selfreport data and, as such, must be viewed with a skeptical eye. According to the selfreported data for the entire school, 120 parents were enrolled in parent organizations, 200 
hours of volunteer service were carried out by parents, 5 parents served on the school council or its committees, $\mathbf{7 5}$ parents indicated they voted in school council elections, and 225 students claimed their parents had attended at least one teacher conference during the preceding year. Specific details as applicable to DEMS are not available. (See Appendix B for complete report.)

\section{Student Outcomes}

Qualitatively, neither Rural Values (RV) nor Parent Religiosity (PR) was deemed to have much impact on Student Outcomes (SO). According to the subjects interviewed for this study, a rural background and a connection to religion were inconsequential to SO.

Rural Values (RV) and Student Outcomes (SO)

A few parents and students felt that going to a larger (urban) school could provide them with more opportunities (usually meaning sports scholarships). One or two students expressed concern that their rural backgrounds limited them in the area of humanities and in their college potential. A few parents agreed with those assessments. On the other hand, some parents upheld rural life as a better foundation for building a better person. Their perceived benefits of living in a more urban area, with larger schools, were negated by concerns about violence and crime and drugs. For those reasons they felt that the RVSO connection was positive.

Parent Religiosity (PR) and Student Outcomes (SO)

Because the Parent Religiosity Affiliation Bond (PR-ABOND) is considered so strong, it would seem requisite to investigate its relationship to SO. Throughout the duration of this study, notes were made concerning the quality of the educational 
experience each student received while a student at DEMS as perceived by the parents.

According to the RFN, many parents felt that the religious background or foundation of a child was important to a child's school success. "Parents who take their children to church care about them. They are more interested in them. They are more willing to help them do well in school." "If you go to church, you want to do good. That's part of being a Christian. So that carry's over into school." The pastor interviewed for this study echoed that sentiment: "Christians are taught to be good parents. To bring their children up right. If you bring them up right, you are going to see that they do their best in school." $8 \mathrm{MH}$ parent, who is well educated and is the pastor of a local church, reflected: “A child's education is usually only as strong as a parent's support. And a parent who makes sure his or her child is involved in church is going to make sure that child is supported in school." (See Appendix X.)

$8 \mathrm{HH}$ parent, $8 \mathrm{MH}$ parent, and 7MM parent are certain there is a positive relationship between religion and good performance in school. According to 7HHs parent:

There's definitely a connection between religion and valuing education. I think most church kids have more respect for each other and for teachers. They have better behavior and do better in school. They are more active in school if they are active in church. Church helps a kid want to do more and do better because they have a better value system.

On the other hand, some perceive the strong relationship between religion and school as negative. 7ML parent feels, "There is one textbook that taught Christianity and against Jehovah teaching, but religion should be left up to the churches." Another parent who had a child in the seventh grade felt that school was invasive to their belief system. She said they had as little to do with school as possible. They obeyed the laws of 
attendance, and that was it. School, she felt, was for the temporal self and not for the eternal self. Another parent had children in both the seventh and the eighth grades. She also considered the school to be an interference with their conservative beliefs in the ways certain subjects were taught. She objected to the teaching of the Greek and Roman gods and to the teaching of evolution. But she also felt the school offered her children a substandard level of education. She felt they would obtain a better education through home schooling, so she subsequently withdrew them to home school them with a conservative Christian program.

Regarding of the PR-SO relationship, the custodian's comments synthesize the views held by those who perceive the effects as negative. She states:

Around here religion is more important than academics. People are less involved in public school. Sometimes parents will even pull their kids out of school. They think they can provide everything a kid needs at home. Parents are not usually college graduates, but they feel confident that a one-on-one contact will compensate.

\section{Parent Involvement (PI) and Student Outcomes (SO)}

With exception of only a few, most parents (RFN and interviews) could not get beyond their sense of the "cold school atmosphere" to assess a parent's involvement in a child's education at the middle school. Many parents cited the disdain voiced by their children for their participating at school and/or chaperoning for various programs. Added to the "rejection" that many experienced at DEMS, most parents simply believed that active involvement was not an option. There was, therefore, little qualitative data gathered to evaluate the effect of Parent Involvement (PI) on Student Outcomes (SO).

\section{Summary}

This study is a case study of 109 students and their parents from a small rural, 
middle school community in Western Kentucky. The study used mixed methods (quantitative and qualitative) and followed a concept model designed by the author (Figure 1). Ten Research Questions were developed to reflect Demographic Factors (Socioeconomic Status, Family Structure, and Specially Designed Instruction), Independent Variables (Parent Values--five Rural Values and five Parent Religiosity), Mediating Factor (two Parent Involvement), and the Dependent Variable (Student Outcomes--GPA).

Quantitative results include descriptive statistics reported for a small $N(38)$. Psychometric analyses were required to reduce the number of variables because of the small $N$. Optimal scales yielded Parent and Student Combined responses for nine of twelve scales. Attempts to combine the separate constructs produced a final set of scales for the regressions: a set of five Rural Values, one composite Parent Religiosity variable, and one Parent Involvement variable. Variables were developed from Figure 1--each arrow representing one of the ten Research Questions. Regressions revealed that only SDI and Anti-Intellectualism influenced GPA. The small $N$ and possible biased sample (restricted range due to higher SES respondents) may have been related to the fact that more variables were not significant.

Qualitative results were used to supplement data generated by the quantitative part of the study. Interviews, document analysis, and researcher field notes provided information for the Qualitative section. 


\section{CHAPTER V}

\section{DISCUSSION}

While framers composed the United States Constitution in the 1800's, mandating a separation of church and state, clergy educated children throughout the colonies in ecclesiastical structures, allegiance to God was established in prayers before Congress, white crosses of remembrance were erected in government cemeteries, and the Ten Commandments were sculpted for government buildings. In reality, religion has always been an integral part of our system of education and governance, and religion continues to play a major role in values, protocol, and rules of behavior--written and unwritten (Silk, 2000), especially in the South. Given added prominence by the prevailing conservative political rule, Christianity plays a major role in the lives of many people schools were created to serve. Without the acknowledgment of that role, there is risk of losing access to a significant resource in the high stakes game in which teachers and administrators can lose their jobs and students can lose a better future. In order to meet standards set by the Kentucky Education Reform Act (1990) and the No Child Left Behind Act (2000), educators will need to take advantage of every possible resource. Religion and rusticity may provide a previously overlooked option for assistance.

Decades of research have provided evidence of the link between ecclesiastical forces and parental values (Ellison \& Sherkat, 1993; McNamara, 1985; Peshkin, 1986; Sherkat, 1997) and between religiosity and rural values (Ellison \& Sherkat, 1995; 
Peshkin, 1986). Abounding research clearly identiflies the relationship between parent involvement and student outcomes (Brandt, 1989; Epstein, 1995).

On the other hand, rural communities in states like Kentucky (which ranks 47th in adults graduating from high school and that contends with contemporary reputations for moonshining, feuding, good old boys, hillbillies, and rednecks) (Klotter, 2000) display obvious need of research into education and its relationships to parent values and ruralness. Such data, however, are lacking. The American Educational Research Association announced that their 2003 conference, presented to more than 10,000 educators for which "the list of sessions in the program book was more than 300 pages long" (Strange, 2003, p. 4), offered only sixteen out of 7,000 research papers. Researchers from Appalachia Educational Laboratory (2004) call for "high-quality research on rural education issues" without which "important questions remain unanswered and successful strategies and policies remain unidentified" (p. 3), and Hoffman and Miller (1997) call for more quantitative and qualitative research into social issues that involve church-state relations (such as parent values and education). Thus is the basis for this study.

The Study in Brief

The author examined the overlapping spheres of rural values, religiosity, parent involvement, and education in small, rural, Southern, middle schools. After an extensive literature review, a central research question emerged: How do the Interrelationships of Parent Religiosity, Rural Values, and Parent Involvement relate to Student Outcomes in a southern, rural, middle school in Kentucky? After the author considered participantobserver status, she established the format of the study. According to Maykut and Morehouse (1994), culture-sensitive research requires that the study be appropriate to the 
gender, education, training, and relevant personal experiences of the researcher. They further contend that the researcher be able to understand, through looking at words, actions, and records, patterns which emerge from the data and to discover contextual findings rather than generalizations.

Those conditions met, the author also met stipulations suggested by Bell (1993). In order to study a society or culture in depth, a researcher must depend "heavily on observation" while maintaining "complete or partial integration into the society being studied," for the researcher "has to be accepted by the individuals ... being studied" ( $p$. 10), becoming an "interactive spirit, force or principle" and "one with the study" (p. 25). Also, Smith (2002) concludes that a case study is more effective when it is place-based because the author studies something important to his/her life; thus, the environment for the study becomes the setting--the "living laboratory" (p. 589). Finally, according to Strong (1995), curiosity arousal is necessary for learning to occur, and in order for the curiosity to be aroused, a topic needs to relate to one's personal life: "The connection cannot be superficial; it must involve an issue or idea that is both manageable and unresolved" (pp. 10-14).

In light of all of these findings, a case study of mixed method (quantitative and qualitative) was set in the author's home school in a small southern, rural, middle school. A theoretical model (Figure 1), developed by the author, guided the study. To provide the quantitative data for the study, the author developed and administered a questionnaire of Likert and Likert-type items for students and parents of the school. To form the qualitative foundation of the study, the author selected and interviewed participants from questionnaire respondents, staff of the school, and other community members. 
Researcher Field Notes (RFN) and document analysis provided additional information to establish contextual findings. Data for Student Outcomes (SO), the dependent variable, were Grade Point Averages (GPA) drawn from the high school (where the students later moved) guidance counselor's records.

\section{Discussion}

The discussion that follows is organized by the major section of Chapter IV. The quantitative analysis includes Descriptive Statistics, Psychometric Analysis of Scales, and the separate research questions, based on multiple regression. Brief findings and analysis are separated for these sections. For the Qualitative Analysis, results and discussions are integrated, consistent with qualitative procedures.

\section{Descriptive Statistics}

This case study of 109 middle school students yielded only $N=38(35.9 \%)$ from the survey. The low return raises incern as to the representativeness of the sample. The participants in this exploratory study were higher than the statewide average in educational attainment (Strange, 2000). Only 11\% failed to graduate from high school, $37 \%$ had a high school diploma, $34 \%$ had taken some college, and $8 \%$ had some graduate school. Because the questionnaire hat to be administered by mail, the fact that educational attainment reflected higher levels was not unexpected. Economic levels were largely between $\$ 30,000$ and $\$ 60,000$ per year (66\%), again, not unexpected considering the form of response. It is possible that the reading level and formal nature of the human subjects consent form may have iniluenced the return rate.

It seems likely that these factors combined to bias the actual sample, i.e., respondents were more middle class than the community as a whole. Similarly, the fact 
that $13.2 \%$ of participating students were involved in special education (SDI) instruction supports the expectation that parents of SDI students would be involved in their child's education. For the sample, Student Outcomes ( $M=86.66 \mathrm{GPA})$, and parent income and educational interest levels, were higher than the middle school as a whole, especially considering that SDI students were included in the sample. During checks for possible bias, no differences were discerned across waves of data gathered from original respondents $(N=38)$ and those the researcher gathered during subsequent telephone inquires of non-respondents (71). The lack of differences across this small $N$ does not negate a possibility of a biased sample, especially in light of literature that suggests a strong relationship between SES and parent involvement (Epstein; 1987; O'Neil, 1991).

Participants recorded strong Rural Values responses; three of the five constructs produced means above 3 on a five-point Likert or Likert type scale. The newly articulated Stewardship of Earth merited the highest RV score $(M=4.15)$. The lowest RV score was realized by Social Conservatism/Traditionalism $(M=2.78)$. Responses to all of the Parent Religiosity construct items resulted in means above 3.50. The highest rated construct was Experience with a mean of 3.80, while the lowest rated construct in PR was Ritual, with a mean score of 3.45. It may be notable that the Parent Involvement Belief scale was more rated highly $(M=3.72)$ than Parent Involvement-Action, although, a larger, more representative sample is needed to assess this. In short, it would seem likely that, at least for PI, these scores might be higher than for the school as a whole, but, again, the scores might have been different with a more representative sample.

\section{Psychomøtric Analysis of Scales}

One limitation of this study is the small $N$. Figure 1 conceptualizes three 
Demographic control variables, a set of Parent Values (five Rural Values and five Parent Religiosity), Parent Involvement (two scales) as a mediating variable, and GPA as the criterion Student Outcomes. For the Parent Values and Parent Involvement scales, data were collected in parallel for the parents and students. This produced 24 separate scales plus the three demographic factors--27 independent variables in a data set with $N=38$.

Extensive psychometric work was required to reduce the number of variables. First, parent and student data were examined and combined where possible. For the Parent Religiosity and Parent Involvement constructs, this worked well; Cronbach's (1951) alpha for all of these combined scales demonstrated adequate reliability (greater than .7). The five Rural Values scales required more work. For three scales, an item had to be removed (Anti-Intellectualism, Ethnocentricity, and Stewardship of Earth). In addition, for three of the five, the parent or the student data were not viable and had to be dropped, resulting in a Student only scale, Good Old Boy, and a Parent Only scale, Social Conservatism/ Traditionalism and Ethnocentricity. Even with these changes, two of the scales required a relaxed standard of alpha (greater than .6) (Bernstein \& Nunnally, 1994).

After reducing 24 Parent Values and Parent Involvement variables to twelve, there were still fifteen independent variables. A further data reduction strategy was to combine the five Rural Values into an overall Rural Value composite, and similarly for the five Parent Religiosity and two Parent Involvement scales. This effort resulted in five separate Rural Values constructs, a composite Parent Religiosity, and a Composite Parent Involvement, for a total of ten independent variables. These ten variables (plus GPA as criterion) were utilized for the multiple regressions to answer the ten research questions. 
For Research Questions 1-6, stepwise regressions were utilized. Only significant variables from these analyses were carried forward to the more complex Research Questions 7-10, where hierarchical regression was used. Analysis

Several of the ten independent variables warrant comment. Most studies related to education utilize free/reduced lunch status as a measure of socioeconomic status (SES). However, as Moore (2003) notes, this is a gross measure of social class that does not distinguish between levels of affluence. Further as Smith, Neff, and Nemes (1999) demonstrate, the accuracy is often suspect. In this study, parental education and income were combined into an interval scale. The results, however, indicated that this SES measure had no influence on GPA, although it did affect several of the values scales. Unknown is whether the measure was problematic, or whether the truncated sample, as discussed above, was related to the non-findings for SES. One possibility is that parental education and income would be better analyzed separately, because of the possibility that mining jobs in the area produce higher incomes than typically associated with lower levels of education. Combining parent education and income would confound these two factors.

Similarly, the Family Structure measure utilized in this study produced minimal effects. Although the ratio of dependents to "caretaker labor" is intriguing, and to the author's knowledge, unique in the field, the indicator produced essentially no effects. Again, it is unknown as to whether this is related to the measure itself or to the truncated sample.

As for the value scales constructed in the areas of Rural Values, Parent 
Religiosity, and Parent Involvement, the results for this study are intriguing as well, but not much more can be said. With a single case study, with very small $N$, and with a likely biased sample, nothing can be concluded about their viability as research scales. Several of the scales revealed interesting correlations with one another, with the demographic factors, and with the criterion GPA. Further research is warranted for all of these constructs

It is also worth noting that these were new scales, constructed for this study, based on the research and the author's knowledge of the region. It is not uncommon for new scales to have to undergo psychometric adjustments before they reach a final form, as happened with the five Rural Values scales. Finally, to the author's knowledge, collecting data in parallel for parents and adolescents is not a common practice, if at all. The finding that nine of the twelve scales developed for this study could be collapsed to Parent and Student Combined data (i.e., the responses were highly consonant) is worth continued study in the areas of rural values and religiosity.

\section{Research Question 1}

How do Demographic factors relate to Student Outcomes?

Of the Demographic Controls investigated, only Specially Designed Instruction demonstrated a statistically significant effect on SO (measured by GPA). SDI was found to have a significant negative effect on SO. Socioeconomic status and Family Structure were not related to GPA. Analysis

Kentucky is one of the top ten states considered in urgent need of attention in areas of education, poverty, low teacher pay, and low levels of adult educational 
attainment according to the Educational Climate Index (Strange, 2000). So it is somewhat unusual that SES did not manifest as a significant factor in SO (White, 1982). However, the fact that the study was perhaps inadvertently biased in its sample provides a tentative reason for the non-relationship of SES to SO in this study, i.e., a truncated, higher SES set of respondents. Also, Special Education students would be expected to score lower on measures of student achievement so it is not unusual that factor had an effect on SO. Because this is no separate measure of aptitude in this study, SDI would have served as a gross proxy for ability.

\section{Research Question 2}

How do Demographic factors relate to Parent Values: Rural Values and Parent Religiosity?

Several of the five Rural Values, plus Parent Religiosity, were related to the demographic factors. Family Structure scores were positively related to Social Conservatism/Traditionalism. This means that the lower the parent-to-child resources are, the more likely the SC/T scores will be elevated. In addition, SDI scores were related to GOB. On the other hand, Ethnocentricity and Stewardship of Earth scores were linked to SES. Anti-Intellectualism was not significantly related to any of the demographic variables. For Parent Religiosity, both SES and SDI were influential. Analysis

These findings are consistent with current literature, which is replete with examples showing that living in the Rural South predisposes one to be a conservative, Christian, Republican (Silk, 2000) who likes guns and hunting and who follows the patterns of male supremacy and the Good Old Boy system (Graham, 2002). Yet this does 
not explain why the Good Old Boy scores were linked to special education status. Both Ethnocentricity and Stewardship of Earth were positively linked to SES. For Ethnocentricity, this is a surprising result. It is generally expected that such values are associated with class and racial resentment, particularly for lower/working class whites who see minorities surpassing them socioeconomically (see Della Fave, 1980, on relative status deprivation). Perhaps in the conservative rural South, the phenomenon is more widespread. Little (1998) found that an overall Rural Values score, including both Ethnocentrism and Social Conservatism, was negatively associated with affirmative action issues.

The positive connection for SES and Stewardship of Earth may reflect more understanding and appreciation of the need/calling to protect the earth and related environmental issues. However, because this is a new scale, further work is needed to confirm such speculation.

The last of the five Rural Values, Anti-Intellectualism, is the most intriguing. None of the demographic factors were significantly related. Perhaps the best way to explain this is to posit Anti-Intellectualism as a generalized cultural phenomenon in the rural South, rather than a trait associated with social class.

Coreno (2001) goes as far as suggesting that fundamentalist Christians can be considered a class unto themselves, based on education, income, occupation, education, and traditionalism. Regnerus (1998) finds that fundamentalists tend not to be involved in social issues or activities. In fact, non-Christians are more likely than anyone to give to the poor. This would lead one to expect fundamentalists to be less involved in the school despite encouragement from ecclesiastical leaders, such as Baptists and non- 
denominationalists (Wingfield, 1994), "to help teach people to read and write ... the Bible ... to benefit them in this life and the next" (p. 1). All of this would be consistent with Hofstadter's (1963) anti-intellectualism.

The other Parent Value investigated in Research Question 2 is Parent Religiosity. The positive relationships for both SES and SDI are puzzling. Considerable literature (Bowman, 2001; Glock, Ringer, \& Babbie, 1967) confirms that religiosity tends to become more intense as socioeconomic standing declines, yet here the association was positive. Again, this finding may be related to the truncated and higher SES sample for this case study.

As for the positive association between Parent Religiosity and having a special needs student, perhaps more religious families come to see this as a special calling, that God has challenged them to care for this child. On the other hand, the very condition of having a special needs child, in itself, tends to lower a person in social stature. For this reason alone, a parent might need to seek compensatory fulfillment through religious connections (Glock \& Stark, 1965). This is certainly speculative, but to the author's knowledge, this positive relationship has not been previously found in the literature. More work is warranted on this possibility.

\section{Research Question 3}

How do Demographic factors relate to Parent Involvement?

Table 21 revealed a significant positive relationship between SES and higher PI levels.

This result is consistent with previous research. Educators universally lament that those who come to parent-teacher conferences, particularly for middle and high school 
1996). As Coreno (2001) has pointed out, the demographic factors in the rural South set the stage for a lower income class. This fact indicates the increased need for both parents to work--discouraging parent involvement in schools. That, coupled with the prevailing religious conservatism, suggests that unsuccessful education and correction of other social ills is not a priority. Fundamentalist Christianity offers infallible solutions to all problems. The goal is to "save" everyone and that will cause the social ills to be corrected as a secondary outcome (Bowman, 2001; Peshkin, 1986; Watt, 2002). That this fundamentalism is typically inversely related to SES (Glock et al., 1968) supports this finding.

Analysis

\section{Research Question 4}

How do Parent Values (Rural Values and Parent Religiosity) relate to Student Outcomes?

Of the Rural Values constructs, only Anti-Intellectualism related significantly to SO, in a negative direction. The other type of Parent Values, Parent Religiosity, was positively related to student GPA (see Table 22).

Analysis

Hofstadter's (1963) thorough treatment of Anti-Intellectualism in the rural South has been confirmed by this small case study. Graham (2002) notes an "anti-merit attitude [,] . . simply not [accepting] merit as a cultural value" (p. 96).

However, the positive relationship between Parent Religiosity and GPA represents a complex set of forces. On the one hand, conservative Christians exhort their members to become literate so they may spread God's word and so that they can advance 
His kingdom. On the other hand, fundamentalists tend to disapprove of the typical public school curriculum, often developing their own for home school use and for schools under their design (Peshkin,1986; Watt, 2002). These thoughts are buttressed by the supplemental interviews in which parents reflecting higher religious concerns and scores were those who also expressed stronger belief in PI and stronger vocalization of their encouragement for higher student achievement. Of course, one must be reminded of the small $N$ and the possible misinterpretation based on the restricted range for this case study.

\section{Research Question 5}

How do Parent Values (Rural Values and Parent Religiosity) relate to Parent Involvement?

The only Rural Value showing a significant relationship to PI, based on the stepwise regression, was Stewardship of Earth, positivel linked. Likewise, the relationship between PR and PI was positive. The Adjusted $R^{2}$ for both factors was 27 . Analysis.

In this case study, the small $N$ necessitated data reduction to the extent possible. Both Parent Involvement scales--Belief and Action--were combined into an overall PI construct. But the Action scale $(M=3.00)$ lagged behind Belief $(M=3.72)$ on the descriptive results. It may be that many parents who believe in parent involvement do not have or do not take the time to be actively involved. Subsequent studies should separate the two constructs to see if they have differential impact.

The positive relationships for both RV-Stewardship of Earth and Parent Religiosity with Parent Involvement raise interesting questions. Graham (2002) says the 
Southern 3 R's are rasin,' rastlin,' and runnin'--not very encouraging in a parent's decision to become involved in education. In addition, Hoge and Carroll (1978) say that, while volunteering for church programs is higher in conservative peoples, volunteering within the community occurs more often in mainline Protestant denominations. Yet, during the supplemental interviews students stated outright that they did not want parents involved in any school activity other than sporting events. Are more religious parents less likely to accede to adolescent peer pressures? Another possibility is the truncated, higher SES sample for this study. Clearly more investigation is needed.

As for the positive correlation between Stewardship of Earth and Parent Involvement, there is little guidance from the literature. Examination of the items in the Stewardship of Earth scale (see Appendix R) does reveal a reverence for the land and for its care for future generations. Again, however, to the author's knowledge, this construct has not previously been utilized as a scale. That, coupled with the small and likely biased (higher SES respondents) sample in this study, clearly suggests that more study on this issue is needed.

\section{Research Question 6}

How does Parent Involvement relate to Student Outcomes?

No significant relationships were revealed during the regressions. Analysis

Researchers have documented that parent involvement, of any kind for any length of time, does have a positive relationship to student outcomes (Brandt, 1989). That parents equaled their beliefs in Parent Involvement with their actions to support that belief is not supported by this case study. The mean scores for Parent and Student 
Combined were PI-Belief $(M=3.72)$ and PI-Action $(M=3.00)$. As suggested under

Research Question 5 (immediately above), the non-findings may be related to differential importance for what parents believe versus what they $d o$. Subsequent research should examine these constructs for their separate effects on student outcomes.

Another possibility exists. Again, related to the small sample for this case study, Parent and Student responses were combined. Although the combined coefficient alpha for PI was .751 , there is the possibility that analysis of parent only and student only could yield different results (the reader should recall the statement from the supplemental interviews about students not wanting parent involvement other than for sports).

Finally, the small and potentially biased sample (higher SES) may again be a possible explanation. The mean GPA (SO) for students involved in this study (86.66) is much higher than the average for the entire middle school. That fact in itself would signify that more involved parents both responded to the survey and had children who achieve higher.

\section{Research Question 7}

How do Parent Values (Rural Values and Parent Religiosity) relate to Student Outcomes, controlling for Demographic factors?

Hierarchical regression was used to investigate this question. Only variables significant in Research Questions 1,2, and 4 were entered into this equation. Specially Designed Instruction (SDI) and SES were the demographic factors while AntiIntellectualism and Parent Religiosity were the Parent Values forwarded. The results demonstrated that only SDI and RV-AI were significant in the final model, with $37.3 \%$ of the variance in GPA explained. 
Analysis

In this final model, the greatest influence on student outcomes was special education status, a proxy for student aptitude. As noted throughout this discussion, the non-findings for SES are likely related to the small and relatively affluent respondents, a restricted range that would reduce the significance of social class. After accounting for SDI, Anti-Intellectualism increases the Adjusted $R^{2}$ by .063 . This negative influence (stronger AI values, lower GPA) is consistent with Hofstadter's (1963) classic study, particularly the strength of this trait throughout the rural South. That SES was not related to Anti-Intellectualism suggests that this is a diffused cultural phenomenon rather than a value that is based in class differences.

\section{Research Question 8}

How does Parent Involvement relate to Student Outcomes, controlling for Demographic factors?

For this hierarchical regression, only special education status (SDI) was carried forward from Research Questions 1, 3, and 6. Among the demographic factors, Research Question 6 demonstrated that Parent Involvement was unrelated to Student outcomes. Thus, the hierarchical equation reduced to only one demographic factor, SDI, that was entered into the equation. The result was identical to Table 15 for Research Question 1: SDI had a negative influence on GPA and explained $31 \%$ of the variance. Analysis

Parent Involvement was not related to student GPA in this study, a result likely because of the low $N$, and the inadvertent biased sample of higher-than-average students. Therefore, the results remain inconclusive. A more representative sample could perhaps 
lend support to prior research (e.g., Epstein, 1987; Henderson, 1987).

\section{Research Question 9}

How do Parent Values (Rural Values and Parent Religiosity) relate to Student Outcomes, as mediated by Parent Involvement?

The hierarchical regression for this question is based on the results from Research Questions 4, 5, and 6. Two Parent Values--RV-Anti-Intellectualism and Parent Religiosity--were linked to Student Outcomes. The mediating factor, Parent Involvement, was not significantly related to student GPA. Thus only RV-AI and PR were entered into this equation. The result is identical to Table 22: Anti-Intellectualism is negatively related to GPA while Parent Religiosity has a positive influence. Together the two variables explain $17 \%$ of the variance. Analysis

The analysis for Research Question 9 simply mirrors the results for Research Question 4. Because the mediating factor in Figure 1 (Parent Involvement) is not significantly related to Student Outcomes in this study, that variable was not forwarded to this hierarchical regression. Similarly, the two Parent Values that were significant for PI-Parent Religiosity and RV-Stewardship of Earth--also were not forwarded to this equation because there was no possible indirect linkage to GPA. (See Analysis under Research Question 4, above.)

This finding could prove to have powerful implications for faith-based initiatives and the endorsement of using ecclesiastical resources in resource-poor rural areas. If PR proved a positive relationship to higher SO, the potential for rural youths to obtain competitive gains in achievement could be greatly expanded--allowing for the 
cooperative endeavors of church and state. The good news, at least for this small case study, is that Religiosity does not appear to be all bad in its relationship to educational achievement. Further research into the degree of conservatism and/or fundamentalism and the degree of educational attainment, consistent with the work of Sherkat and Darnell (1999), is needed. However, as with all findings for this study, the small $N$ and restricted range of higher SES respondents must be considered as strong threats to validity.

\section{Research Question 10}

How do Parent Values (Rural Values and Parent Religiosity) relate to Student Outcomes, controlling for Demographic factors, as mediated by Parent Involvement?

Research Question 10 represents the hierarchical regression for the full model (Figure 1). Demographic controls were entered first, followed by the Parent Values (Rural Values and Parent Religiosity), followed by the mediating variable (Parent Involvement). The dependent variable is Student Outcomes (GPA). But in this full model, only the variables significant from Research Questions 7-9 are forwarded to this final regression. From Research Question 7, the control factor Specially Designed Instruction (SDI) and the Rural Value (Anti-Intellectualism) were significant. From Research Question 9, RV-Anti-Intellectualism and Parent Religiosity were significantly related to GPA when mediated by Parent Involvement. However, Research Question 8 demonstrates that Parent Involvement is not related to GPA.

Thus the variables forwarded to the final regression were SDI (as a control) and two Parent Values (RV-Anti-Intellectualism and Parent Religiosity. The results are identical to Table 24 (from Research Question 7). SDI and RV-AI are both significant, 
with both having a negative effect. Together the two variables explain $37.3 \%$ of the variance on GPA.

Analysis

Graham (2002) speaks of the Rural Value of anti-merit or Anti-Intellectualism (see Hofstadter, 1963), implying that this force would hinder PI and, indirectly, SO. In this study, however, Parent Involvement was not a factor, although Anti-Intellectualism did have a negative effect on GPA, the only variable that did once special education status had been controlled (SDI). On the other hand, there is some evidence that conservative Christians are interested in education. Parent Religiosity was positively related to GPA, although it dropped out of the equation when SDI was controlled. This finding counters Coreno (2001) who notes the negative impact of conservative religiosity on educational attainment.

These findings must be viewed with caution. This case study had a very small $N$ and the sample appears to have been biased (respondents were higher SES than the community as a whole). Thus both the higher social class and restricted range on some variables may well have influenced the direction of findings as well as the nonsignificance of other variables (e.g., social class and parental involvement).

Whatever the effects of the limited sample, these results constitute interesting results on the intersection of Rural values, Parent Religiosity, and Parent Involvement, a topic virtually unexplored in the literature, particularly in the South. It would appear that the conservative religious fold may support education, but it must be according to their own religious values and standards. In fact, Graham (2002) reports that, in northern Kentucky, "an evangelical ministry called Answers in Genesis is currently overseeing a 
\$14-million Creation Museum and Family Discovery Center," bringing it in "under budget," and "through the use of union labor" p. 112. (Also see Qualitative Section, following).

Home schoolers in this community abide strictly by curriculum developed, distributed, and monitored by the state home schooling association, which is led by a conservative Christian pastor [RFN]. Communication with the local assistant superintendent [R. Yarbrough, personal communication, 2002] revealed that the group's curriculum is acceptable by Kentucky's educational objectives and goals. However, at least in the area of science, that would seem a doubtful judgment considering the fact that evolution is completely disavowed.

Thus, there is the possibility that conservative Parent Religiosity here may not have negative influence on SO and PI. As long as the educational system works within the conservative cultural framework, high PR parents appear to be actively involved, at least at home, in their children's education and do appear to support student achievement. Clearly more research is warranted, based on a larger and more representative sample. Also, more work on the connection between conservative religiosity and science curriculum is needed.

\section{Qualitative Analysis}

In contrast to the separation of Findings and Analysis above, the findings and analysis are mixed in this section. They are combined into a narrative format consistent with qualitative analysis, organized by variables.

\section{Demographic Controls}

Because of the geographical isolation of rural areas bus rides can last over two 
hours each way. Some students at DEMS live far into the countryside on roads that are dirt covered and dangerous when it rains. Geographical concerns and distance prevent high levels of Parent Involvement; that situation is exacerbated by the necessity to work due to low SES--both parents work, sometimes two jobs each. During the school year, lower income parents shied away from involvement, just as lower income students seemed to place less value on achievement. In addition, salaries for individuals in rural schools are lower in every category and "create a competitive disadvantage in recruiting and training high qualified teachers and effective school leaders" (Strange, 2004, p. 1)-teacher salaries in Webster County are among the lowest in the state.

Child-to-parent ratio established the Family Structure (FS) score for this study. For the most part, those who responded to the survey reflected a low FS score (meaning there were as many parents as there were children in the home). These results were not surprising because it was expected that the parents with lower FS scores (higher family resources) would be the more involved and more willing to respond to the survey. However, one family reported a high FS score, meaning there were many more children than there were adults to supervise their care. In addition, one of the siblings, confined to a wheelchair because of cerebral palsy and mentally challenged as well, was assigned to a special education class at another county school located about twenty miles away. These parents were rarely seen at DEMS except for required visits to the SDI room where this child also received support.

\section{Rural Values}

Throughout the course of this study as the author worked as an ethnographer, one theme emerged consistently: members of the rural community recognize how they are 
perceived by their cosmopolitan neighbors as less intelligent and less cultured (see Appendix Y) and they very much resent it. Ridicules, jokes, parodies (see Appendix Z), songs, and even political cartoons about southern rural dress, music, art forms, intellect, dialect, and religiosity are recalled as artillery in the new "cold war" of words (see Singham, 2000). During the interviews, subjects tended to step aside from the verbal bullets, responding with chuckles or skewed glances to reminders of insults. At times they responded with half-hearted non-sequiturs, for even they sense that the caricatures are accurate somehow. So long have they been the underdog in this new cold war, they seem to have adopted the posture and demeanor of a prisoner of war. In light of this perceived atmosphere, Parent Involvement seems to suffer.

Wlodkowski and Ginsberg (1995) find that learning occurs more readily in an environment that includes and values a student's culture. Student respondents in this study were ready to decry their texts because they were so removed from their own lives (See Strong, Silver, \& Robinson, 1995). Moreover, ethnographers say that the South offers little in the way of "cultural enlightenment" opportunities (Graham, 2000, p. 29), and the W. F. Kellogg Foundation released a report (Strange, 2002) that confirms rural communities as behind the times and severely lacking in cultural activities. True enough, Webster County has no theater, no movie stadiums, no skating rink, no bowling alley, no museum, no community college, no youth community center, and no dancing facility. For students in this resource-poor community, the school and the churches provide the few opportunities for development. Because of the ubiquitous religious involvement, if religious parents are not tapped as resources, the level of parent involvement inevitably must suffer. 
Placed-based education is not new (Smith, 2002), but academicians remain largely unaware of its significance. However, as revealed in the review of literature, learning is easier, more complete, and greatly enhanced when the concepts being taught are embedded within cultural context. Thus, southern rurals are captives in a land where it is illegal to discriminate against almost every variation of human condition except for being obese or being from the rural South. In light of this, they know discrimination first hand as they struggle to digest curricula that have been developed without understanding of or regard to their culture. They strive to compete on achievement tests designed to assess their knowledge of a culture that does not honor their own.

As a result, at least in part, of negative attitudes towards rural-isms, Michael Graham (2002) claims, "Southerners have largely rejected the idea of abstract intellectualism, a.k.a. "book learnin"” (p. 20) while they laud real learning as, "What you get from the hands-on experience of working on machines, planting crops, stumpbreaking cattle, etc.” In other words, rurals might say, “'Boy, you got plenty of book learnin, but you ain't got the sense God gave a pissant"' (p. 20). This position was reflected often by respondents during formal interviews and in casual conversations. At school, book-oriented students were called "dweebs"; most students, who considered themselves average, outwardly disliked and avoided the dweebs. Even "lower" students seemed to like those who were athletic standouts, pop culture aficionados, rich, or beautiful people. Students, however, who could achieve both in academics and also one of the "acceptable" arenas were granted higher status by their peers. Because parents were undoubtedly encouraged to conduct the athletic programs (because the teaching staff was not compensated, they declined involvement), sports was the area in which they 
became involved, not in the academic program.

Graham (2002) goes further to claim Southerners embrace passion but reject reason. "New Yorkers pretend they've read books they haven't. Southerners deny reading the ones they have" (p. 37). He says they claim to be "the dumbest people at the table" in any conversation. It is true that many books considered acceptable by the National Council of Teachers of English, even award-winning adolescent novels, were removed from the library shelves at DEMS because of their sexual content--regardless of the depth of the narrative. One removed novel simply referred to the mating of pigs; another was a resource book that included sexual diagrams for human intercourse.

Anti-Intellectualism may be left behind as a rural moves from the South to other regions, seeking to further education or to develop a non-rural career. However, upon return to the rural South, he or she quickly learns to put city ways to bed in order to survive the resentment of rural folk. Whereas a young black urban youth might learn that to act intelligent is to act "white," (Ogbu, 1992) a young rural youth learns that to act intelligent is to act "urban."

The Anti-Intellectual limitations are not limited to youth, however. As was heard more than once in the teacher's lounge and among faculty during lunch break, "Getting any degree higher than a master's degree around here is like committing professional suicide. You become over-qualified and they don't want you around" (J. Willoughby, personal communication, 2002).

Ethnocentricity is valued in this community as Graham (2002) reports it to be in the rural South. This is a closed community. People of color, of obvious ethnic characteristics, with different sounding names, with foreign dialects, or people who 
simply do not "fit" (Little, 1998) are suspect. Even after years of living in the community, a person may be considered an outsider $(8 \mathrm{HM})$ and may not be allowed into social circles. Insiders commonly ask questions that seek to determine one's genetic connection to the community: "Who's your daddy? Is Alvin your cousin? Was your mama the sister to Billie Joe? Didn't your grandmother own the Markwell farm? Was that your nephew's obituary in the paper last week? Just how are we related?" They may spend hours straightening out and establishing relationships. These tendencies dampen involvement of parents who "don't belong."

Graham (2002) refers to rural Southerners as a race-obsessed ethnic class dedicated to maintaining their supremacy. Though this author cannot support Graham's position, she can honestly say that this community is segregated; its members are mostly white; they hold racial biases; and they make prejudicial statements that would be inappropriate to repeat here. During the interviews, some of the parents outwardly expressed a relief of having no real racial integration with which to contend, one of those tacit factors one will rarely admit directly. But, as Lortie (1975) notes, indirect questions will sometimes elicit information that penetrates more deeply to one's core values. The interview question had nothing to do with race, but the racial connection crept into the answer. Little's (1998) study also reported negative responses to race that surfaced in her primarily rural sample.

Especially repugnant to the typically southern rural mind in this area is homosexuality. No matter how promiscuous an adulterous man might be, for example, despite a possible transgression of one of the Ten Commandments, his behavior is considered less abhorrent that the actions of a monogamous homosexual. Homophobia 
underscores the conservative structures of this local society. A local college discussion group became heated one evening as the talk turned to homosexuals. A rather passionate young male student exclaimed, "I'd like to take everyone of 'em, cut off their genitals, and th'ow 'em in the ocean." He received unrestrained applause. Likewise, a "city slicker" or practitioner of a foreign religion (even Catholicism stretches the bounds of acceptance) is at risk of remaining a "stranger" after as many as thirty years (L. Tucker, personal communication, 2002). In addition, parents hesitate to become involved in learning or to support curriculum, that does not support locally endorsed values, e.g., tolerance of multiculturalism is frowned upon.

Though many of these characteristics might seem to be more associated with Community-Centrism (Little, 1998) (see Chapter II where Ethnocentricity and Community-Centrism were explained and combined), the extremity of the loyalty with which the rural southerner holds them or exhibits these values pushes the parameters toward the "ethnic" end of the descriptive spectrum. Moreover, from the perspective of those outside the South, there seems little doubt as to the "ethnicity" of a hillbilly. His dress, his haircut, his leisure activities, his language and grammar, his music, his dance, his values, and his comedy are denigrated and held up as a laughable state of affairs. Listening to late night television would leave little doubt as to the places of origin most maligned. For example, all Jay Leno, needs to do to be assured of a laugh is to mention that a guest is from Kentucky or Tennessee.

The Social Conservative/Traditional construct in the community can be detected after only brief verbal intercourse. (See Appendix AA for typically conservative, antigovernment sentiments.) The conservative standard for the submissive female is alive 
here, supported by the numerous Baptist churches--men and women believe that a wife is to be subservient to her husband (Smith, 2000). The mission statement of the Southern Baptist Convention boldly states: "A wife is to submit herself graciously to the servant leadership of her husband even as the church willingly submits to the leadership of Christ" (Graham, 2002, p. 168).

Rurals have "more traditional values than their urban and suburban peers," (Strange, 2002, p. 2) and the fact that the rural tendency to be "intolerant of outsiders and difference" (p. 4) and others of their own culture is a concern which may interfere with learning new things or about different people. For example, DEMS students did not have access to sex education classes. Graham (2002) says, "In the South, it's okay for a kid to have a gun, but giving him a condom might inspire inappropriate behavior" (p. 38).

Needless to say, if a teacher encourages academic achievement through drastic, rapid change, he/she can offend parents and discourage progress. The closed society and mono-cultural attitudes (Ethnocentricity) interfere with Student Outcomes because one finds it difficult to learn what one does not understand and does not respect.

Stewardship of Earth was difficult to assess during this study. First, it has been articulated only in the most tenuous terms in related literature. Second, the vacuity of common language through which to discuss the concept has rendered it unwieldy, almost impossible to capture in an instrument such as a questionnaire or formal survey. Third, the connection to the land seems so natural, so innate, it is not easy for the residents to objectify. Young farmers who have left the land to attend school, or to seek a career in a more urban setting, are perhaps the most skilled at verbalizing the SE construct. They compare the land to part of themselves or part of their spirits, parts they could not escape 
even though they did not realize it until they had moved away. The land is often what brings them back despite guarantees of poor wages, hard work, insane hours, and no financial benefits or security. (See Appendix BB.)

An illustration is a man who had left the farm because of financial necessities; he obtained employment in a large metropolitan area as a well-paid plant manager; he married and had a family. Yet, after more than twenty years of absence, the main sold his city belongings and returned to the family farm where he said, "I couldn't wait to get back" (RFN). For others, talking about SE was too difficult, seemingly because of the inability to articulate and because of the fear of dishonoring it. A parallel could perhaps be made between SE and the blood flowing through a person's veins: it is not noticed, it cannot really be described because it cannot be readily seen, and it is taken for granted until it is spilled and becomes separate from the person who possesses it.

"Tending the land" is no accident of language. Farmers tend the land as carefully as they do their children, often putting its needs before those of the family. Caring for a demanding, often fickle and unforgiving piece of land can build character and strong work ethics. Plus, efforts at conservation, disease and pest control, and squeezing every possible ounce of production out of every acre are incentives for learning. On the other hand, prioritizing farm work over homework, missing school to plant or harvest a crop, and trivializing all knowledge other than farm-necessary are some of the negative aspects of SE. In addition, the low income inherent in family farming creates lower SES, lower Parent Involvement, and lower opportunity levels for the children who live there.

The GOB construct represents a system of acquiring employment through who one knows rather than what one knows. Graham states: "As a young man, I knew that 
half the people working in my rural public school were incompetent cousins of wellplaced politicos" (p. 18). Paula Little (1998) refers to elements of this construct as "employee fit." It does seem in some instances the concept of "fit" plays out in the DEMS community. For example, at the time of the author's study, the central office was made up of an unusual number of individuals who had come from one particular locale in the county or who had direct ties to those individuals. Among those were the superintendent, the assistant superintendent, the director of pupil personnel, the director of transportation, the federal programs director, the director of special education, and several other lower level employees. The situation was, in fact, a subject of faculty room speculation.

County government is also undeniably male dominated--county judge, county attorney, sheriff, jailer, county assessor, bank presidents, preachers, retail owners, etc. As well, it is the males who run the family farms and households for the most part. Because females here defer to men with little, if any, resistance, most of the GOBs are male. Despite the fact that the district judge is female, she is said to have obtained her position because of who her family is and because of all they know about "dirt" on everybody else. She has continued to hold on to her job, according to local informants, in spite of her excursions (published in a neighboring county newspaper) into DWI, drug use at parties, and an occasional arrest for being in possession. In addition, her husband and his friend were brought before her on drug charges as she presided in court. They reportedly received no punishment.

GOB in action includes the local crop chemical plant that stores and sells hydrogenous ammonia (commonly used in the production of methamphetamines). On a 
regular basis, the night watchman calls the plant supervisor to the site because "a leak" has been noticed. Upon the supervisor's arrival, he finds that as much as $80 \%$ of the tank has "escaped" due to a "faulty" valve. A few times when State Troopers have arrived on the scene unexpectedly, they have discovered several suspicious clues (hoses, nursing equipment, coolers, and containers). Only rarely have those reports gone farther.

Although it can be said that the Good Old Boy system can work for females, it is predominantly a system for good old boys, a variation on Graham's (2002) “A-Spermative Action" or anti-merit attitude (p. 96). It is a power system, but the male power ascendancy can have more to do with elements (understood fully only by those inside the GOB fraternity) such as status symbols endorsed by the GOB culture: drag racing, tobacco spitting, pickup trucks, country music, hunting, guns, and John Deeres.

Community youth stage drag races on country back roads, especially on weekend nights, for bragging rights to the fastest car or the greatest risk-taking. The remnants of such activity are left on the roads in the form of "rubber laid down," skid marks, and white quarter mile, half mile, and mile markings on the pavement. Some country fellows claim that the drag racing is a throw back to the moonshiner runs when the fastest car and driver tried to outrun the local police with a fresh load of "home brew." NASCAR originated in the South and the major racetracks are mostly in southern states. Local folk purchase many items sold to honor their selected NASCAR heroes at the nearby WalMart stores and they wear their "numbers" on their jackets and caps.

Young and old GOBs alike reportedly compete to cram as much tobacco into their jaws as they can without gagging when they are sharing camaraderie. After a while of proper chewing, they hold informal tobacco-spitting contests to see who can spit the 
biggest mouthful of dark-colored saliva the farthest. Their pickup trucks often bear the brunt of on-the-go spitting with dark splotches on the doors and windows.

The pickup trucks are status symbols, too. The relationship between a man and his pickup has been duly memorialized in one of Joe Diffie's biggest hits, "Pickup Man" (Phillips, 1994). Though the scene is played in various ways in different locations, the script does not change much when the talk turns to the GOBs and their pickups. On the farm, GOBs gather either before or after work, during lunch, or on weekends to exchange verbal jousts, vying for supremacy in several GOB-important areas, including the category of the pickup truck. The most recent version of the game centers around the new diesel trucks that offer a microchip that can be added to the computerized engine to boost the horsepower. The bigger the engine, the longer the wheelbase, the larger, longer, and heavier the load, and the higher the gas mileage, the better--either in reality or in embellished re-tellings. "A four-wheel-drive, three-quarter-ton, heavy duty truck with a towing package, extra large radiator, and extra-heavy-duty springs is the one to have!" boasts one of the men (L. Tucker, personal communication, 2002). "We also want to get the most cattle to market in a single load or pull the biggest piece of equipment out of the biggest mud hole or snow drift."

One area family is a five-generation farm family, a father and three of four sons having lived on the same land for about 75 years on land within about a 25 -mile radius of each other, on about 14,000 acres between them. From the beginning, they selected John Deere for their brand loyalty. Today, many years and hundreds of machines and millions of dollars later, they still buy only John Deere equipment despite the fact that it is often much more expensive. Anyone who ventures away from Deere to purchase another 
brand becomes the target of heavy-duty embarrassing remarks. When five grandsons began farming, they, too, chose the Deere. When four granddaughters married, their husbands converted to a life of farming and also farmed with the Deere. Though the loyalty in the area has begun to wane, a drive through the farmland reveals more Deere equipment than any other brand. In addition, farm family members own and display John Deere caps, shirts, jackets, belts, playing cards, key rings, cook ware, mail boxes, yard ornaments, clocks, lawn mowers, and wind chimes. A Deere is a status symbol that has been popularized in the song "John Deere Green" by Joe Diffie (Linde, 1993).

Rural living usually means long hours of labor intensive work, but on certain dates, whether a crop is being planted or not, everything seems to stop. Farmers climb off their equipment and pick up their weapons, for the GOB is also a hunter. To underscore the importance of this event, it needs to be said: cropping rarely stops to allow for human experiences--not birthdays, not weddings, not births, not graduations, not athletic events, not dance or piano recitals--sometimes not even for funerals. But for the opening day of deer season, the equipment is silent. Regardless of tests/projects, homework, or state assessments, if it is opening season for deer hunting, males will expect to be absent from school. Needless to say, fathers will not be helping out at school on opening day of hunting season.

Of course, there are other seasons that follow (several more deer seasons using different types of weapons, rabbit season, squirrel season, quail season, dove season, turkey season, duck season, etc.). Sometimes the GOBs will leave the state to hunt for bear, moose, elk, or alligator.

All of the hunting requires guns--guns of varying sizes (calibers) with varying 
accessories and abilities. The men have special slings that hang in the pickups to carry the guns. They buy or build large wooden display cabinets to show them off in their homes. Ching and Creed (1997, p. 29) find that "it is increasingly important not just to own guns, but to display them and aggressively defend their ownership." On occasion, males spend money on guns to the detriment of their family. For example, $8 \mathrm{HHh}$ reported being required to go to the store to collect a cold check she had written. It wasn't until she obtained her balance statement from the bank that she learned why her check had "bounced." Mr. 8HHh had purchased a new, expensive, high-powered rifle on his last day before being laid off from his part-time job as a security man and had neglected to inform his wife before deer season (see Green \& Silk, 2004).

To say there is educational benefit in the GOB culture might be stretching the truth, but there is a great deal to know about different types of firearms and an extreme amount of sophistication in modern farm equipment--which can cost over a quarter of a million dollars each. Conversely, school work, school attendance, and Parent Involvement do seem to lose out during planting, harvesting, and hunting seasons. Parent Religiosity

A description of this community could not be written without reference to its religiosity. Interviews and RFN confirm what researchers say: Southern rurals tend to believe in religion; their actions tend to confirm that they are religiously conservative. Furthermore, they report high levels of the experiential realm of religiosity: revelation of God, inner messages, spiritual awakenings, born again experiences. Some respondents recorded charismatic experiences: speaking in tongues, slaying in the spirit, holy laughter, or miraculous healings. 
However, there is some disconnect in the carry over from belief to ritual (actions), for it is evident that reports of church attendance and contributions are higher than the actual incidences themselves $(8 \mathrm{HHh})$. It seems that religiosity here may have more to do with what goes on in the inside rather than what a person does on the outside. Preachers do exhort church members to do good works, but those good works are often translated as efforts in leading someone else into "saving grace" or "accepting Christ as Lord and Savior." The primary purpose of religion is to gain a believer admission into the "next" life and not to help him/her in "this" life. Breaking traffic regulations, cheating on income taxes or spouses, cultivating an occasional marijuana, or producing a little "meth" on the side are not necessarily considered bad--unless someone else finds out about it (L. Papineau, personal communication, 2002).

This is consistent with evangelical conservative Christianity in the striving to look "inward" instead of "outward." According to a Southern Baptist minister who was born and reared here, saving souls is considered of greater importance than doing good works. These attitudes have led to more local, less overseas charity. The mission field has, in effect, been moved and many foreign based missionaries have found themselves cut off, without funds or support, living in previously maintained foreign mission fields (D. Duncan, personal communication, 2002).

Rural churchgoers relate to the image of the Norman Rockwell painting that some still have on the walls of their homes. It portrays a good, common folk, a poor rural family gathered around a table, holding hands, saying a blessing. A child brought up in a "good Baptist family" can often remember hearing the verse from the Bible that says if you have two shirts, give the other one to a brother. Children from a poor rural, 
conservative-religion, Southern home were expected to observe that vow of pious poverty unless, until, all of his/her close kin could be taken to the "rich house" with him/her. The value of "pious poverty" can be detected in conservative, rural churches in the South even today. What money they do have is likely to remain within the local congregational pool, being used for local congregation needs: buildings and grounds, preacher salaries, and denominational missions--not used for school needs.

In addition, though many hold an understandable expectation that Christians are to "do good," participation in social causes is not necessarily a requirement of Conservative religious culture. One needs only to note a quote from Southern Baptist preacher, Jeff Devine, to understand the reason many Conservative churches do not promote involvement in civic activity in order to fulfill a mandate to do good. Devine exhorts, "We must remember: religion is not to do good, or to promote good, even though that may be, probably will be, an outcome. The purpose of religion is to recognize and establish the authority of God over man. The Bible itself admonishes us to withdraw from the world--be not one with the world" (J. Devine, personal communication, 2002). In spite of the fact that Christians feel Biblically called to read the "Word," keep God's day holy, confess sins, accept Christ as Lord and Savior, profess Christ as Lord before others, be baptized in Jesus' name, testify to others what Christ has done for them, and to go "into all the world, teaching and baptizing" in Christ, when all is said and done, for many of members of the rural community, a person does not even have to go to church or to read the Bible to be a Christian. All that is really required is to believe and to try to behave (A. Brown \& L. Papineau, personal communication, 2002). So the impetus to remedy social ills such as illiteracy is not bolstered by religious forces. 
On the other hand, whether the individuals are active participants in a church or not, religiosity is represented in WWJD (What Would Jesus Do?) bracelets that students wear to school, WWJD license plates on vehicles, large crowds of youths who travel from one church to another for successive weeks of vacation Bible school (VBS) during the summer months, fall and spring church revivals (week-long church meetings dedicated to "reviving" membership and "saving lost souls"), talent competitions that feature gospel singers, Habitat for Humanity projects, and the popularity of televangelists and radio preachers. Also, prayers are offered before sports events, school board sessions, and PTA meetings, and a weekly sermon is prominently printed in the local newspaper. Webster is a "dry" county (no alcoholic beverages can be sold within county boundaries--legally, that is). Finally, it is pro-Bush country, part of the Christian Right territory (Rozell, 2003) (also see Green \& Silk, 2004, and see Appendix CC).

It may be said that indications of religiosity are numerous and that religiosity is strong because this is the South. However, it may also be said that religiosity is strong because this is rural America; other than school sporting events, there are few other community offerings for social exchange or cultural enhancement. Simply said, other than school, there is church or there is not much of anything else. If the school rejects Parent Religiosity, the "other life" of a parent seems un-welcomed at school.

\section{Overlapping Spheres: Rural Values and Parent Religiosity}

At the outset of this study it was the author's objective to investigate the intersect of public education and parent religiosity. It became apparent early on, however, that in some degree religiosity and Rural Values overlapped. Indeed, it became evident that, in some cases, Rural Values were the religion and that Parent Religiosity and Rural Values 
would be virtually impossible to separate. (This was somewhat validated by interviews and correlations conducted during the study.) Perhaps the most interesting aspects of this study are the revelations garnered when considering all of the values discussed in their "overlapping spheres" context, as the total is often greater than the sum of its parts.

First, male supremacy promoted in scripture is reflected also in the structure of rural families. Inside and outside church, men are the head; their needs take priority; they lead the prayers; they are preachers and leaders; they preside at meals while women prepare the food and serve; they receive recognition for work completed by the women.

Second, Stewardship of Earth has a traceable religious origin, all the way back to St. Francis of Assisi and his exhortations to be good stewards of the land and animals. The biblical promise of a certain tract of land to the Jews lends an undercurrent of legitimacy to those expressing a feeling of belonging to the land or it being a part of the farmer. Tending the land can become a holy calling of sorts. The inability of local farmers to generate sufficient income from their tilling the soil plays into clerical admonitions of pious poverty alluded to in sermons.

Third, one of the more intriguing aspects of the overlapping spheres is that of salvific-love music, both in church hymnals as well as in country music. Central to this salvific music are family, home, love, faith in God, blood of the lamb, salvation, transformation, sacrifice, and poverty (see Grossman, 2002). However, there is no mention in country music or in conservative Christian hymnals of ethnic diversity or homosexuality despite the pleas from both for love, peace, and understanding.

Fourth, most poignantly, Anti-Intellectualism is embedded in the rural community and in the rural church. Whereas the rural mind reacts rebelliously because of the 
disrespect aimed at rural culture, the churchgoer is reminded that too much knowledge is what got Adam and Eve kicked out of the Garden of Eden. After all, it was fruit from the tree of knowledge that Eve ate. So, according to church teaching, too much knowledge can be a dangerous thing. It can lead to arrogance and to self-dependence and away from reliance on prayer and dependence on God: "Lean not unto thine own understanding" (Proverbs 3:5). Any potential ecclesiastical buttressing for Parent Involvement can thus be all but nullified.

\section{Parent Involvement}

With little reservation, parents interviewed felt that Parent Involvement is an important element of their child's education. That said, it is also clear that the parents who responded to the invitation to interview were those who were more involved than most in their children's education. Moreover, even these parents felt PI should occur early in the child's school career, not as late as during middle school. At the middle school level, few parents are involved.

A few DEMS parents claimed the school atmosphere as cold and uninviting, and several parents denied a sense of welcome from the principal. (The principal's behavior toward a number of teachers seemed to confirm this depiction.) Some teachers were discouraged from enrolling parents in programs or projects that required at-school involvement. Yet, contrary to these perceptions, the principal spoke of his open-school policy to parents at parent-teacher conferences and made statements during faculty meetings that he practiced an open-door policy for parents.

Some parents explained their lack of involvement due to economic and/or work reasons. One parent (7HHs) said she was not smart enough to be involved in classroom 
activities. A few parents cited scheduling. Student responders were not hesitant to explain the lack of PI at school with a repetitive protest: "I don't want them there!"

On the other hand, PI at sporting events is highly participatory; students express acceptance and expectation of PI for sporting activities. Middle school athletics are carried out through parent volunteers, not school staff, so higher levels of PI are understandable. But Anti-Intellectualism could be a factor; for whatever reason, Parent Involvement, other than that connected to sports events, was not given high priority. Research findings that parents will avoid PI unless they feel welcomed was affirmed (Epstein, 1987).

\section{Student Outcomes}

Only the most confident, self-assured students seem able to ignore the lure of Anti-Intellectualism; they are the ones with their eyes set on goals beyond the parameters of the local community. They appear to realize that grades, though not necessarily learning, are important to their success. The remainder find other avenues of affirmation. For some it is sports, for some it is the GOB game, and for some it is the popularity competition that demands personality, looks, or money. For a few it is the fringe culture: the dark, black-clothed, body-pierced, tattooed, brooding clusters reminiscent of bats hovering by the school entrances between classes. For them attending classes is merely a means to the end--the end of school.

\section{Recommendations}

Repetition of the study in other rural settings is recommended. A major weakness of the study is the low return rate $(N=38$ out of 109$)$. A likely consequence of this is a truncated sample, with more involved parents of better students much more likely to 
respond to the mail surveys, i.e., parents who value education less and are less involved in their children's schooling are probably less likely to take the time to complete a survey about their participation in that school-home nexus. More in-depth interviews from other school communities within this same county would strengthen internal validity.

Extending the study to other rural school districts would determine whether the patterns revealed generalize to larger populations. For that matter, these themes could well have an impact in urban areas as well. For example, a powerful force in the Black community is religion. Further investigation of insider documents of the area churches might be helpful in substantiating portions of this study into religiosity.

In this study, Parent Religiosity, Rural Values, and Parent Involvement were examined in relation to Student Outcomes as defined by GPA. However, GPA is prone to local norms, grade inflation, influence from ability grouping, etc. SO as defined by a standardized achievement instrument or state accountability data could have better interpretive value. Also, Ellison and Sherkat's (1997) recommendation to research the relationship of varying parent religiosities to long-term student outcomes is affirmed as a need. More broadly, findings in this study were congruous with Hoffmann and Miller's (1997) call for more quantitative and qualitative research into social issues that involve church-state relations.

\section{Policy and Practice}

The rural South is a place where religiosity and everyday values overlap. It is a place where high court judges go to jail to protest the removal of a Ten Commandment's monument from the court house. It is a place where the Confederate flag is proudly hoisted almost 200 years after the end of the Civil War. It is a place where students pray 
around the flag pole at school, where fire arms are encouraged and abundant even for preteens, where moonshine still flows, where the death of a NASCAR hero garnered almost as much attention and outward show of grief as the 9-11 World Trade Center bombings. It is a place where the only social gathering spot besides school is church.

To a significant degree, the quantitative portion of this study, field notes, interviews, and literature all reveal an overlap of Rural Values (RV) and Religiosity (PR) in the Rural South. Based on that, it can be said that the constructs and sub-constructs of RV and PR come into play here. In Socially Conservative/Traditional cultures, change comes slowly and painfully, the people display xenophobic tendencies, curriculum controls from outside are viewed as a threat, and the integration of religion (creationism) into schools is perceived as good. (It might be said that this was confirmed by the "clear conservative mandate" of the 2004 election, particularly in the South). In such a setting, it is perhaps a primary task of exploratory research to discover an informational foundation so that future researchers will know what questions to ask. To that end, during this study, poignant concepts were unearthed and need to be examined further.

One, within Rural Values resides a sense of resentment toward the forces that judge these people negatively, giving rise to a posture of Anti-Intellectualism, that acts in opposition to things seeming more academic or scholarly in nature. This posture may be related to low Parent Involvement. Two, people in this community want to protect their belief system against the threats to devalue their culture, faith, and way of life from the outside world. Because academia presents that kind of threat, Parent Involvement is discouraged. Three, though rarely articulated, there is a tacit understanding of Stewardship of Earth and its religious underpinnings. A question could also be raised 
about the connection between Anti-Intellectualism and rebellion southerners felt about the relinquishing of land ownership at the end of the Civil War and the bitterness and resentment that still seems to run deep. But there is no evidence to support this line of thinking, and future study could yield better understanding of the issues concerning Stewardship of Earth.

Four, a hierarchical power structure, with parallels to cronyism and nepotism, known commonly as the Good Old Boy system, rewards not what one knows, but who one knows. (The literature credits the South with the Good Old Boy concept, but a system of power, rules, rewards and punishments, and strategies for advancement seems to exist in many regions, although the names, the specific rules, the rewards and punishments, the particular means of working the system may be different according to local norms, consistent with culture, ethnicity, and place.) If academic achievement (SO) places a premium on what one knows, and Parent Involvement is seen as merely supportive of that, there is a lesser need seen for PI.

Five, Parent Religiosity-Belief runs strong among rural natives. Rarely has that been a serious consideration when planning curriculum or Parent Involvement programs in this community. Six, previously accepted elements of religious ritual are not necessarily consistent with standards here. For example, to establish a Ritual score for a person who abides by the hallmarks of religious conservatism in the Rural South, one might need to include other items in a Ritual measurement: (a) selecting a personal hero (or an individual from history one would most want to meet) from the Christian domain, (b) performing "other worldly" religious acts, e.g., winning a "soul to Christ," (c) listening to salvific love songs in country music, (d) reading religious fiction or watching 
religiously colored television programming (Touched by an Angel, Seventh Heaven, Joan of Arcadia), (e) buying religious tapes and books, (f) playing religious games, (g) owning religious texts or religious periodical subscriptions, (h) attending religious conferences/training, (i) working in Vacation Bible School, (j) participating in revivals or church musicals, (k) performing good deeds, (l) wearing religious symbols (e.g., crosses, tee shirts), or (m) distributing religious tracts. Seven, geographical isolation and economy of cultural offerings promote Primary Affiliation and Affiliation Bonding with school and church.

Other areas of potential study for policy and practice within the realm of Parent Involvement include the following issues: (a) Anti-Intellectualism as a result of socially accepted prejudice against ruralness (rednecks), (b) the socializing forces of religiosity in the rural South, (c) the relationship of Parent Religiosity to Parent Involvement in southern rural schools, (d) the loss/gain in social capital (Coleman, 1988), i.e., Parent Involvement, as a result of exclusionary attitudes toward conservative Christianity while promoting multicultural respect for other religious cultures, (e) re-grounding in the neutrality stance of the Constitution regarding church and school, and (f) gain/loss to educational outcomes as result of faith-based initiatives.

\section{Future Research}

Based on this study, the following areas hold promise for continuing and future research:

1. Rural Values and Parent Religiosity as inexorable constructs and their relation to one another and to Parent Involvement

2. The relationship between the different Rural Values and Parent Religiosity constructs, particularly Anti-Intellectualism, and Student Outcomes. 
3. Rural students as minority students.

4. Higher SES and Religiosity across the spectrum of social class and race and in urban areas.

5. Parallels in social, political, economic, and religious conservatism.

6. Primary Affiliation and Affiliation Bond and their relation to social capital (Coleman, 1988).

7. Salvific love music and its relation to Rural Values and Parent Religiosity.

\section{Conclusion}

Before a conclusion can be drawn, a consideration of the schism between Parent Religiosity and the scientific method needs to be articulated. It is that schism that serves as a pivotal issue in parents' decisions to become involved (whether or not to participate and best strategies) in their children's education--especially in the rural South. Without articulating a rational, objective perspective of the cleft between the two seemingly dichotomous concepts, one would have difficulty reaching a truly accurate conclusion about the overlapping spheres of Rural Values, Parent Religiosity, and Parent Involvement in southern rural education. This fact became more poignant throughout this exploratory case study.

\section{Religiosity and the Scientific Method}

In a study of the science/religion debate in education, brought to life by the evolution/creation controversy, Mano Singham (2000) suggests that the lines that separate science from religion can be drawn between four subgroups: elite science, popular science, elite religion, and popular religion. The first is science, purely and completely. The second includes belief in the ability of extraphysicalities (witches, magicians, superstitions, etc.) to violate the laws of science. The third sees the creator as 
a force that set up the universe and the laws of nature, then stepped aside, rarely, if ever, intervening (mainstream religions). The fourth, which makes up fundamental religious faiths, holds to a personal faith in a personal god who can be prayed into intervention at will. But Singham's main point is that the lines of demarcation between the subgroups are fuzzy--there are scientists who also have elite religious beliefs, there are elite religionists who believe science is merely the laws of god put into motion. Singham points to Stephen Jay Gould, the paleontologist who claimed that science cannot confirm or deny elements from the religious sphere, and to noted religious leaders who claim that the Bible cannot be interpreted literally for scientific explanation.

Ultimately, Singham (2000) proffers the stance of social constructivists: "All knowledge, including scientific knowledge, is filtered and interpreted through the lenses of the observer and is thus inevitably colored by those lenses.... The unfiltered truth ... is thus impossible to discern" (p. 431). This means that "modern scientific knowledge is the product of the people (primarily male and Western) who were involved in its creation and that there might be equally valid alternative scientific world views" ( $p$. 431). To carry this line of thinking one step further, it may follow that science is a kind of religious faith in the dogma that has been established by previous scientific leaders-just as the flat-earth or earth-centric beliefs were based on faith in scientific "knowledge." In fact, to deny the existence of God without evidence to prove that denial, is a betrayal in itself of the scientific method. Denial of something it cannot disprove is a paradoxical statement of scientific dogma. (On the other hand to accept on faith the existence of something for which there is no empirical evidence violates scientific principles.) Singham concludes: the attack on Christian religiosity "may be backfiring on science. 
Rather than coming across as reasonable defenders of the scientific world view, these scientists risk being perceived as arrogant elitists who sneer at those who do not understand them and adopt a scorched-earth policy in dealing with those who disagree with them" (p. 432).

With this point in mind, it can be said that every culture has religious foundations and education cannot be devoid of a culture's religious foundations. American history is inseparable from religion--immigration by Europeans based on the desire to worship as they chose, the Christian foundations of the Declaration of Independence, and the establishment of the common school. World history and humanities include units that cover Greek and Roman gods. The study of World War II could not be complete without a discussion of the Jews and Hitler's treatment of them. Multicultural studies mandate an overview of differing religions. Some educational philosophers think that to disallow study that includes a discussion of religiosity of any kind is an egregious intolerance of Christian heritage, and that to avoid discussions of religions in America is a betrayal of the neutrality stance of the First Amendment. Moreover, morals and character education contribute to the common good and are necessary for democracy to survive.

On the other hand, history has shown that when total governmental power is vested in a particular "breed" of religion, and that when the separation of church and state is not only breached but is also dissolved, that democracy is threatened. This fundamental principle assumes that the purpose of education is not to cause students to become more or less religious, nor is it to encourage them to become more or less conservative or fundamental in their faith; it is to prepare children to survive, to produce, and to enhance the cultures to which they belong. It is to help them in their efforts 
toward becoming responsible, contributing members of this democratic society with respect for the multicultural foundations of all of its members--including the Christians. Rural Values, Parent Religiosity, and Parent Involvement

It is evident, through researcher field notes, document analyses, interviews, and, to a significant degree, by the quantitative portion of this small case study, that AntiIntellectualism is an active force, at least in this community where to achieve is to be "urban." Hofstadter's (1963) qualitative and historical study concerning AntiIntellectualism as an impediment to educational achievement of some rural students is affirmed. As well, it is evident that Rural Values, Religiosity, and Parent Involvement overlap in this southern rural community. What is not clear is whether or not negative attitudes toward southern Rural Values, in themselves, perpetuate Anti-Intellectualism. Of particular interest here, however, is the fact that none of the demographic factors, including socioeconomic status, had a significant effect on Anti-Intellectualism. This suggests a diffuse cultural phenomenon rather than a trait that is grounded in social class distinctions.

One needs only to recall history to realize that entities outside the South have been largely unsuccessful at forcing southerners into submission or remedying them of their rural/religious heritage. The region that seceded once from the union and even today claims that it "shall rise again!" maintains a cohesive identity and strongly rebellious nature.

To bow to all of the southern rural Christian demands to alter curricula to reflect their views could be catastrophic to the integrity of, at the minimum, standard science education. But the trend in this region (from ten only five years ago, to 58 in 2004) to 
withdraw children from public school for home schooling is disconcerting. Although there are no statistics to assess how many of these withdrawn children have been removed from the public schools because of religious "intolerance," there are advocates within the local churches who encourage others to follow suit. Refusing to lend at least a gracious bow to their requests for a respectful status of some of the tenets of their faith (juxtaposed to the contemporary Muslim women's demand to wear their complete bodycovering garment for driver's license pictures), could further alienate and antagonize them into further Anti-Intellectual rebellion.

Presently in Kentucky, the state department of education maintains a "hands off" policy when it comes to monitoring most home schools. Even though on paper there is an attendance requirement, those records are rarely checked (parent interview 8HHh), and curricula are almost never reviewed. Only when a home schooler wishes to return to public school is he/she assessed according to local placement standards. These policies, along with the home school movement, the Anti-Intellectualist fervor, and the Republican (current powers that be) agenda of faith-based initiatives and vouchers, could result in such decreased funding that the outcomes for the public school system could be disastrous.

Therefore, those who hope to advance the cause of educational potential for children in the rural South will need to work from within the culture to increase Parent Involvement and Student Outcomes. Otherwise, the risks to public education in the South could be amplified through increased resentment and subsequent reactionary moves such as home schooling and Christian schools. (Home-schooled or parochial students are not necessarily at educational risk, although the quality of scientific curricula 
is certainly open to debate. However, because of the consequential decrease in financial support for the public schools, students who are left behind will suffer.)

In the final analysis, Rural Values and Parent Religiosity in the South mandate the tapping of Christian cultural resources, including that of Christian Parent Involvement, in the provision of the optimum educational experience for the students in the schools there. Otherwise, southern rural students are denied, not only the best education possible, but also the respect due them as part of the multicultural world in which they live. 


\section{REFERENCES}

Appalachia Educational Laboratory. (2004). Finders keepers: policies to help rural schools recruit and retain high-quality teachers. The Link: A Publication for Education Practitioners, 23(2), 1-3.

Advice desired--Louisiana court--good-old-boy system--what can be done? (2002). Retrieved February 19, 2003, from http://www.childcustodyattorney.com/ Louisiana/_disc37//000000081.htm

Allison, P. D. (1999). Multiple regression: A primer. Thousand Oaks, CA: Pine Forge Press.

Asa, L. F. (1995). The psychology of religious commitment and development. Lanham, MD: University Press of America.

Babbie, E. (1990). Survey research methods (2nd ed.). Belmont, CA: Wadsworth.

Bader, C., \& DeMaris, A. (1996). A test of the Stark-Bainbridge theory of affiliation with religious cults and sects. Journal for the Scientific Study of Religion, 35, 285-303.

Bahr, H. M., \& Martin, T. K. (1983). "And thy neighbor as thyself"? Self-esteem and faith in people as correlates of religiosity and family solidarity among Middletown High School students. Journal for the Scientific Study of Religion, $22,132-144$.

Bailey, G. D., \& Smith, N. J. (1987). The rural school administrator's role in achieving sex equity of curriculum. The Rural Educator, 38(9),1-6.

Barber, R. J., \& Patin, D. (1997). Parent Involvement: A two-way street. Principal Leadership: Journal of National Association of Secondary School Principals: Schools in the Middle, 5(Suppl, 9), 31-33.

Batson, A. B. (1993). Having it y'all: The official handbook for citizens of the South and those who whish they were. Nashville: Rutledge Hill Press.

Bauman, L. J., \& Adair, E. G. (1992). The use of ethnographic interviewing to inform questionnaire construction. Health Education Quarterly, 2, 9-23. 
Becker, H. J., \& Epstein, J. L. (1982). Parent Involvement: A survey of teacher practices. Elementary School Journal 83(2), 85-102.

Bell, J. (1993). Doing your research project: A guide for first-time researchers in education and social science (2nd ed.). Philadelphia: Open University Press.

Bellah, R. N., Madsen, R., Sullivan, W. M., Swidler, A., \& Tipton, S. M. (1996, March). Individualism and the crisis of civic membership. The Christian Century, 260265.

Bjarnason, T. (1998). Parents, religion and perceived social coherence: A Durkheimian framework of adolescent anomie. Journal for the Scientific Study of Religion, 37, 742-754.

Bourdieu, P. (1986). The forms of capital. In J. Richardson (Ed.), Handbook of theory and research for the sociology of education (pp. 241-258). Westport, CT: Greenwood Press.

Borland, M. V., \& Howsen, R. M. (1999). A note on student academic performance in rural versus urban areas. American Journal of Economics and Sociology, 58, 537546.

Borg, W. R., \& Gall, M. D. (1989). Educational research: An introduction. White Plains, NY: Longman.

Bowman, R. M., Jr. (2001). The word-faith controversy: Understanding the health and wealth gospel. Grand Rapids, MI: Baker Books.

Boysen, T. (1992). Transition plan: Bridging the gap from Kentucky high schools to workforce, post secondary education, or military. Frankfort: Kentucky Department of Education.

Brandt, R. (1989). On parents and schools: A conversation with Joyce Epstein. Educational Leadership, 47(2), 24-27.

Brookover, W. B., Beady, C., Flood, P., Schweitzer, J., \& Wisernbaker, J. (1979). School social systems and student achievement: Schools can make a difference. New York: J. F. Bergin.

Bulletin board. (2003, February 6). Journal-Enterprise, (Providence, KY), p. 2.

Caldwell, E. (1961). God's little acre. Athens: University of Georgia Press.

Carlson, R. V. (1992). What does it mean to work in a rural setting? A study of rural influences. Journal of Rural and Small Schools, 5(1), 41-47. 
Ching, B., \& Creed, G. (Eds.). (1997). Knowing your place: Rural identity and cultural hierarchy. New York: Routledge.

Christ, G. (1995). Curriculums with real-world connections. Educational Leadership, 52(8), 32-35.

Church, A. H. (1993). Estimating the effect of incentives on mail survey response rates: A meta-analysis. Public Opinion Quarterly, 57, 62-79.

Church, G. J. (1993, November 22). Whatever happened to the great American job? Time, 34-39.

Clark, R. (1995, October). Casting parents in leading roles. Kentucky Teacher, p. 6.

Cohen, D. L. (1995, May 3). Joining hands. Education Week, pp. 35-38.

Cohen, P. (1995). Schooling away from school. ASCD Education Update, 37(6), 1, 6.

Coleman, J. S. (1988). Social capital in the creation of human capital. American Journal of Sociology, 94(Supplement), 595-5120.

Collins, H. T., Czarra, F. R., \& Smith, A. F. (Eds.). (1998). Global education: Challenges, cultures \& connections [Special issue]. Social Education, 62(5).

Coreno, T. (2002). Fundamentalism as a class culture. Sociology of Religion, 63, 335360 .

Cornwall, M., Albrecht, S. L., Cunningham, P. H., \& Pitcher, B. L. (1986). The dimensions of religiosity: A conceptual model with an empirical test. Review of Religious Research, 27, 226-244.

Creed, G. W., \& Ching, B. (1997). Recognizing rusticity: Identity and the power of place. In B. Ching \& G. W. Creed (Eds.), Knowing your place: Rural identity and cultural hierarchy (pp. 1-38). New York: Routledge.

Crocker, L., \& Algina, J. (1986). Introduction to classical and modern test theory. Fort Worth, TX: Harcourt Brace Javonovich College Publishers.

Cronback, L. J. (1951). Coefficient alpha and the internal structure of tests. Psychometrika, 16, 297-334.

Crouch, R. (1995). New realities. Kentucky State Data Center Newsletter, Frankfort: Kentucky State Data Center.

Cushman, K. (1993, May). Essential collaborators: Parents, school, and community. Horace, 9(5), 1-8. 
Cwick, S., Wooldridge, D., \& Petch-Hogan, B. (2001). Field-based teacher education for greater cultural sensitivity. The Rural Educator, 23(1), 14-17.

Darnell, A., \& Sherkat, D. (1997). The impact of Protestant fundamentalism on educational attainment. American Sociological Review, 62, 306-315.

Data bank. (1994, April). NEA Today, 26.

Davies, D. (1996, July 10). The 10th school: Where school-family-community partnerships flourish. Education Week, pp. 44, 47.

Davis, N. J., \& Robinson, R. V. (1996). Religious orthodoxy in American society: The myth of a monolithic camp. Journal for the Scientific Study of Religion, 35, 229245.

Della Fave, L. R. (1990). The meek shall not inherit the earth: Self-evaluation and the legitimacy of stratification. American Sociological Review, 45, 955-971.

Detwiler, F. (1994). A tale of two districts. Educational Leadership, 51(4), 24-28.

DeYoung, A. J., \& Laurence, B. K. (1995). On Hoosiers, Yankees, and mountaineers. Phi Delta Kappan, 77, 105-112.

Dillman, D. A. (1978). Mail and telephone surveys: The total design method. New York: John Wiley \& Sons.

Din, F. S. (1998). The operations of Kentucky rural schools. The Rural Educator, 20(2), 15.

Dixon School Based Decision Making Council. (2000). School Report Card. Frankfort: Kentucky Department of Education.

Dobratz, B. (2001). The role of religion in the collective identity of the white racialist movement. Journal for the Scientific Study of Religion, 40, 287-301.

Djupe, P. A., \& Grant, J. T. (2001). Religious institutions and political participation in America. Journal for the Scientific Study of Religion, 40, 303-314.

Dorahy, M. J., \& Lewis, C. A. (2001). The relationship between dissociation and religiosity: An empirical evaluation of Schumaker's theory. Journal for the Scientific Study of Religion, 40, 315-322.

Dornbusch, S. M., \& Ritter, P. L. (1988). Parents of high school students: A neglected resource. Educational Horizons, 66, 75-77.

Dryfoos, J. (1988). The rise of the full-service community school. The High School 
Magazine, 6(2), 38-42.

Elam, S. M., Rose, L. C., \& Gallup, A. M. (1993). The 25th annual Phi Delta Kappa/Gallup poll of public attitudes toward the public schools. Phi Delta Kappan, 75, 37-152.

Edmond, E. M., Shipman, M., \& Cahoon D. D. (1992). Religious orientation and locus of control. Psychology--a Quarterly Journal of Human Behavior, 29, 17-19.

Ellison, C. G., \& Andersen, K. L. (2001). Religious involvement and domestic violence among U.S. couples. Journal for the Scientific Study of Religion, 40, 269-286.

Ellison, C. G., \& Musick, M. A. (1995). Conservative Protestantism and public opinion toward science. Review of Religious Research, 36, 245-262.

Ellison, C. G., \& Sherkat, D. (1993). Obedience and autonomy: Religion and parental values reconsidered. Journal for the Scientific Study of Religion, 32, 313-329.

Emerson, R. M. (1983). Contemporary field research. Prospect Heights, IL: Waveland Press.

Epstein, J. L. (1995). School/family/community partnerships. Phi Delta Kappan, 76, 701-712.

Epstein, J. L. (1987). What principals should know about parent involvement. Principal, 66(5), 6-9.

Evans, T. D., Cullen, R. T., Dunaway, R. G., \& Burton, V. S. (1995). Religion and crime reexamined: The impact of religion, secular controls, and social ecology on adult criminology. Criminology, 33, 195-224.

Faria, A. J., \& Dickinson, J. R. (1996). The effect of reassured anonymity and sponsor on mail survey response rate and speed with a business population. Journal of Business \& Industrial Marketing, 11(1), 66-76.

Faulkner, J. E., \& DeJong, G. F. (1966). Religiosity in 5-D: An empirical analysis. Social Forces, 45, 246-254.

Fehrmann, P. G., Keith, T. A., \& Reiners, T. M. (1987). Home influence on school learning: Direct and indirect effects of parental involvement on high school grades. Journal of Educational Research, 80, 330-336.

Feldman, C. (1994, April 30). Survey says parents need big school role. CourierJournal, p. A3.

Fowler, F. J. (1993). Survey research methods (2nd ed.). Newbury Park, CA: SAGE 
Publications.

Fox, A. A. (1997). Ain't it funny how time slips away? Talk, trash, and technology in a Texas "redneck" bar. In B. Ching \& G. W. Creed (Eds.), Knowing your place: Rural identity and cultural hierarchy (pp. 105-130). New York: Routledge.

Foxworthy, J. (1995). Redneck classic: The best of Jeff Foxworthy. Atlanta: Longstreet Press.

Fullan, M. G. (1991). The new meaning of educational change. New York: Teachers College Press.

Gatto, J. T. (1992). Dumbing us down: The hidden curriculum of compulsory schooling. Philadelphia: New Society Publishers.

Garrett, W. (1998) The Protestant ethic and the spirit of the modern family. Journal for the Scientific Study of Religion, 37, 222-233.

Gendall, P., Menelaou, H., et al. (1996). Open-ended questions: Some implications for mail survey research. Marketing Bulletin, 7(5), 1.

George, P. (Ed.). (1998, March). Living with different cultures: Global education Principal Leadership: Journal of National Association of Secondary School Principals: Schools in the Middle, 6(Suppl, 9), 31-33.

Gilchriest, G. (1995). Bubbas \& beaus: From good old boys to southern gentlemen, a close look at the customs, cuisine, and culture of southern men. New York: Hyperion.

Glock, C. Y. (1973a). The dimensions of religious commitment. In C. Y. Glock (Ed.), Religion in sociological perspective: Essays in the empirical study of religion (pp. 9-11). Belmont, CA: Wadsworth Publishing Co.

Glock, C. Y. (Ed.). (1973b). Religion in sociological perspective: Essays in the empirical study of religion. Belmont, CA: Wadsworth Publishing Co.

Glock, C. Y., Ringer, B. H., \& Babbie, E. H. (1967). To comfort and to challenge. Berkeley: University of California Press.

Glock, C. Y., \& Stark, R. (1965). Religion and society in tension. Chicago: Rand McNally.

Graham, M. (2002). Redneck nation: How the south really won the war. New York: Warner Books.

Green, J., \& Silk, M. (2004). Gendering the religion gap. Religion in the News, 7(1), 


\section{$11-13$}

Gregg, S. (1993). Partnerships: Sharing responsibility for children. The Link, 13(3), 1, 12.

Grossman, M. L. (2002). Jesus, Mama, and the constraints on salvific love in contemporary country music. Journal of the American Academy of Religion, 70, 83-110.

Haas, T., \& Lambert, R. (1995). To establish the bonds of common purpose and mutual enjoyment. Phi Delta Kappan, 77, 136-142.

Hansen, R. A. (1980). A self-perception interpretation of the effect of monetary and nonmonetary incentives on mail survey respondent behavior. Journal of Marketing Research, 17, 77-83.

Harp, L. (1993, October). Rebel mom. Teacher Magazine, 25-29.

Hemphill, P. (1974). The good old boys. New York: Simon and Schuster.

Henderson, A. T. (1987). The evidence continues to grow: Parent involvement improves student achievement. Columbia, MD: National Committee for Citizens in Education.

Hersey, P., \& Blanchard, K. H. (1993). Management of organizational behavior: Utilizing human resources. Englewood Cliffs, NJ: Prentice Hall.

Herzog, M. J. R., \& Pittman, R. B. (1995). Home, family, and community: Ingredients in the rural education equation. Phi Delta Kappan, 77, 113-118.

Hill, D. (1989, September/October). Fixing the system from the top down. Teacher Magazine, 50-55.

Hirschi, T., \& Stark, R. (1969). Hellfire and delinquency. Social Problems, 17, 203213.

Hoffmann, J., \& Miller, A. S. (1997). Social and political attitudes among religious groups: Convergence and divergence over time. Journal for the Scientific Study of Religion, 36, 52-70.

Hofstadter, R. (1963). Anti-intellectualism in American life. New York: Vintage Books.

Hoge, D. R., \& Carroll, J. W. (1978). Determinants of commitment and participation in suburban Protestant churches. Journal for the Scientific Study of Religion, 17, 107-127. 
Howard, G. (1993). Whites in multicultural education: Rethinking our role. Phi Delta Kappan, 75, 779-782.

Howley, C. B., \& Howley, A. (1995). The power of babble: technology and rural education. Phi Delta Kappan, 77, 126-131.

Hunter, J. D. (1991). Culture wars: The struggle to define America. New York: Basic Books.

Hust, E. (Ed.). (2003, February 6). The Journal-Enterprise. (Providence, KY).

Iannaccone, L. R. (1990). Skewness explained: A rational choice model of religious giving. Journal for the Scientific Study of Religion, 36, 141-157.

Johnson, J. (1992). Women: A guide to the good old boy's club. Glendale, CA: Griffin Publishing.

Kantrowitz, B., \& Wingert, P. (1992, February 17). An "F" in world competition. Newsweek, 57.

Kelly, J., \& Pope, M. (1995, October). Removing barriers to parent involvement. Kentucky Teacher, p. 3.

Kennedy, D. J. (1986). What's happened to American education. In The rebirth of America (pp. 121-126). Philadelphia: Arthur M. DeMoss Foundation.

Kentucky Board of Education. (1995, October). KBE policy statement: Parent and family involvement. Kentucky Teacher.

Kentucky Department of Education. (1993, February 9). Task force on high school restructuring (Preliminary draft report). Frankfort, KY: Author.

King, M. B., \& Hunt, R. A. (1969). Measuring the religious variable: Amended findings. Journal for the Scientific Study of Religion, 8, 331-323.

King, M. B., \& Hunt, R. A. (1975). Measuring the religious variable: A national replication. Journal for the Scientific Study of Religion, 14, 13-22.

Klotter, J. (2000). Looking backward into the future. Kentucky Humanities, 1, 8-12.

Knapp, C. (1993). Reflecting on the Foxfire approach: An interview with Eliot Wigginton. Phi Delta Kappan, 71, 779-782.

Kober, N. (1996). Do we still need public schools? Washington, DC: Center on National Education Policy. 
Krause, N., Ellison, C. G., \& Wulff, K. M. (1998). Church-based emotional support, negative interaction, and psychological well-being: Finding from a national sample of Presbyterians. Journal for the Scientific Study of Religion, 37, 725.

Kraus, N., Ellison, C. G., Shaw, B. A., Marcum, J. P., \& Boardman, J. D. (2001). Church-based social support and religious coping. Journal for the Scientific Study of Religion, 40, 637-656.

LaHaye, B. (1977). How to develop your child's temperament. Eugene, OR: Harvest House.

LaHaye, T. (1983). The battle for the public schools. Old Tappan, NJ: Revell Company.

Lam, P. (2002). As the flocks gather: How religion affects voluntary association participation. Journal for the Scientific Study of Religion, 41, 405-422.

Let's talk turkey. (2003, February 6). The Journal-Enterprise. (Providence, KY). p. 9.

Linde, D. (1993). John Deere green. [Recorded by Joe Diffie]. On 16 biggest hits: Joe Diffie [CD]. NY: Epic/Legacy.

Lindle, J. C. (1989). What do parents want from principals and teachers? Educational Leadership, 47(2), 12-14.

Little, P. (1998). The effect of rural values on personnel selection practices in Kentucky public school districts. Unpublished doctoral dissertation, University of Louisville.

Little, P., \& Miller, S. K. (2001, April). Rural values and school district personnel practices: An exploration of person-organization fit theory. Paper presented at the American Educational Research Association Annual Meeting, Seattle.

Lortie, D. C. (1975). Schoolteacher: A sociological study. Chicago: University of Chicago Press.

Lott, J. (1995). When kids dare to question their education. Educational Leadership, 52(7), 20-23.

Maddoux, M. (1992). What parents fear most. Eugene, OR: Harvest House.

Marshall, C., \& Rossman, G. (1989). Designing qualitative research. Newbury Park, CA: Sage Publications.

Maslow. A. H. (1943). Motivation and personality. In J. M. Shafritz \& J. S. Ott (Eds.), Classics of organizational theory (3rd ed., pp. 159-173). Belmont, CA: 
Wadsworth Publishing Company.

Mason, E. J., \& Bramble, W. J. (1978). Understanding and conducting research: Applications in education and the behavioral sciences. New York: McGrawHill.

Maykut, P., \& Morehouse, R. (1994). Beginning qualitative research: A philosophic and practical guide. Washington, DC: The Falmer Press.

Mayo, E. (1945). The social problems of an industrial civilization. Boston: Harvard Business School.

Maxim, D., \& Five, C. L. (1997). Parent Involvement: Linking home and school. School Talk, 2(4), 1-3,

McIntosh, W. A., Alston, L. T., \& Alston, J. P. (1979). The differential impact of religious preference and church attendance on attitudes toward abortion. Review of Religious Research, 20, 195-213.

McLaughlin, M. W., \& Shields, P. M. (1987). Involving low-income parents in the schools: A role for policy. Phi Delta Kappan, 69, 56-160.

Meade, J. (1992, May/June). Prodigal parents: To succeed, schools must reach out and bring parents in. Teacher Magazine, 16-17.

Miles, M. B., \& Huberman, A. M. (1994). Qualitative data analysis: An expanded sourcebook (2nd ed.). Thousand Oaks, CA: Sage Publications.

Miller, L. S. (1995). An American imperative: Accelerating minority educational advancement. New Haven: Yale University Press.

Miller, P., Shambaugh, K., Robinson, C., \& Wimberly, J. (1995). Applied learning for middle schoolers. Educational Leadership, 52(8), 22-25.

Miller, S. K., \& Spradlin, L. (1995). Ogbu's theory of castelike oppositional cultures: Generalizing beyond race to class and gender. Paper presented at the annual meeting of the American Educational Research Association, San Francisco.

Mitroff, I. I. (1983). Stakeholders of the organizational mind. New York: Jossey-Bass.

Moore, B. L. D. (2003). An exploration of the influence of demographic factors on individual and aggregate student achievement measurements in the Kentucky accountability system. Unpublished doctoral dissertation, University of Louisville.

NASSP endorses guidelines to help schools and religious communities create 
partnerships. (1999, September). NASSP Newsleader, 5.

Needham, N. (1995, April). Setting standards: How OBE became the three most controversial letters in education. NEA Today, 10-11.

Nitko, A. (2001). Educational assessment of students. Columbus, OH: MerrillPrentice Hall.

Noll, V. H., Scannell, D. P., \& Craig, R. C. (1979). Introduction to educational measurement. Boston: Houghton Mifflin Company.

Ogbu, J. U. (1992). Understanding cultural diversity and learning. Educational Researcher, 21(8), 5-14, 24.

Olson, L. (1989, September/October). A revolution of rising expectations. Teacher Magazine, 56-63.

Olson, L. (1990, August). Prescription for a revolution. Teacher Magazine, 46.

O’Neil, J. (1991). A generation adrift? Educational Leadership, 49(1), 4-9.

Orenstein, A. (2002). Religion and paranormal belief. Journal for the Scientific Study of Religion, 41, 301-311.

Paloma, M. M., \& Hoelter, L. F. (1998). The "Toronto Blessing": A holistic model of healing. Journal for the Scientific Study of Religion, 37, 257-272.

Park, J. A., \& Smith, C. (2000). "To whom much has been given . ..": Religious capital and community voluntarism among churchgoing Protestants. Journal for the Scientific Study of Religion, 39, 272-286.

Pate, R. H., Jr., \& Rooney, P. J. (1994, Spring). Who are these Christians and why are they so angry? ACES Spectrum, 10-11.

The Partnership for Kentucky School Reform. (n.d.). The ABCs of Parent Involvement [Leaflet]. Lexington, KY: Pritchard Committee.

Peavie, L. (1999). A study of collaboration between public school education and home school educators in Kentucky. Unpublished doctoral dissertation, University of Louisville.

Pedhazur, E. T., \& Schmelkin, L. P. (1991). Measurement, design, and analysis: An integrated approach. Hillsdale, NJ: Lawrence Erlbaum Associates.

Perrin, R. D. (2000). Religiosity and honesty: Continuing the search for the consequential dimension. Review of Religious Research, 41, 534-544. 
Peshkin, A. (1986). God's choice: The total world of a fundamentalist Christian school. Chicago: University of Chicago Press.

Peshkin, A. (1994). Growing up American: Schooling and the survival of community. Prospect Heights, IL: Waveland Press.

Phillips, K. K., \& Perdew, H. (1994). Pickup man [Recorded by Joe Diffie]. On 16 biggest hits: Joe Diffie [CD]. New York: Epic/Legacy.

Pinsonneault, A., \& Kraemer, K. (1993). Survey research methodology in management information systems: An assessment. Journal of Management Information Systems, 10, 75-106.

Porter, M. K. (1973). Moving mountains: Reform, resistance, and resiliency in an Appalachian Kentucky high school. In C. Y. Glock (Ed.), Religion in sociological perspective: Essays in the empirical study of religion (pp. 25-29). Belmont, CA: Wadsworth Publishing Co.

Rawson, J. (1989, September 5). What's wrong with American education. Woman's Day, 110-111, 175-176, 180.

Ray, R. E. (1993). The practice of theory: Teacher research in composition. Urbana, IL: NCTE.

Reed, J. S., \& Reed, D. V. (1996). 1001 things everyone should know about the South. New York: Broadway Books.

Regnerus, M. D. (2000). Shaping school success: Religious socialization and educational outcomes in urban public schools. Journal for the Scientific Study of Religion, 39, 363-370.

Religious women achieve more educationally. (1995, December 19). Western Recorder, p. 7.

Resnick, L. (1987). The 1987 Presidential address: Learning in school and out. Educational Researcher, 16(9), 13-20.

Riley, R. (1996). Reaching all families: Creating family-friendly schools. Office of Educational Research and Improvement, U.S. Department of Education.

Roberts, S. (1995). Who we are: A portrait of America based on the latest U.S. Census. New York: Times Books.

Roof, W. C. (1972). The local-cosmopolitan orientation and traditional religious commitment. Sociological Analysis, 33, 1-15. 
Roof, W. C., \& McKinney, W. (1987). American mainline religion: Its changing shape and future. New Brunswick, NJ: Rutgers University Press.

Rosow, L. V. (1991). How schools perpetuate illiteracy. Educational Leadership, 49(1), 41-44.

Rowatt, W. C., Ottenbreit, A., Nesselroade, Jr., K. P., \& Cunningham, P. A. (2002). On being holier-than-thou or humbler-than-thee: A social-psychological perspective on religiousness and humility. Journal for the Scientific Study of Religion, 41, 225-237.

Rozell, M. (2003). What Christian Right? Religion in the News, 6(1), 2-3, 22.

San Diego County Board of Education. (1987). Effective schools curve. San Diego: Effective Schools.

Sautter, R. C. (1994). An arts education school reform strategy. Phi Delta Kappan, 75, 432-437.

Seal, K. R., \& Harmon, H. L. (1995). Realities of rural school reform. Phi Delta Kappan, 77, 119-124.

Schaver. M. (1994, March 23). Petition to repeal KERA presented to legislature. Courier-Journal, p. C7.

Schlafly, P. (1993, November/December). Education: What's wrong with outcomebased education? AFA Journal, 20-21, 23.

Schlechty, P. C. (1990). Schools for the twenty-first century: Leadership imperatives for educational reform. San Francisco: Jossey-Bass Publishers.

Schools: Christians divided over public school involvement. (1999, August 17). Western Recorder, p. 7.

Scheepers, P., Grotenhuis, M. T., \& Van Der Slik, F. (2002). Education, religiosity and moral attitudes: Explaining cross-national effect differences. Sociology of Religion, 63, 157-172.

Schuman, H., \& Presser, S. (1981). Questions and answers in attitude surveys. New York: Academic Press.

Schwadel, P. (2002). Testing the promise of the churches: Income inequality in the opportunity to learn civic skills in Christian congregations. Journal for the Scientific Study of Religion, 4, 565-575.

Sher, J. P. (1995). The battle for the soul of rural school reform: Can the Annenberg 
rural challenge turn the tides? Phi Delta Kappan, 77, 143-148.

Sherkat, D. E. (1997). Embedding religious choices: Integrating preferences and social constraints into rational choice theories of religious behavior. In L. A. Young (Ed.), Rational choice theory and religion: Summary and assessment (pp. 65-85). New York: Routledge Press.

Sherkat, D. E. (1998). Counterculture or continuity? Competing influences on baby boomers' religious orientations and participations. Social Forces, 76, 1087-1115.

Sherkat, D. E., \& Darnell, A. (1999). The effect of parents' fundamentalism on children's educational attainment: Examining differences by gender and children's fundamentalism. Journal for the Scientific Study of Religion, 36, 2335.

Sherkat, D. E., \& Ellison, C. (1997). The cognitive structure of a moral crusade: Conservative Protestantism and opposition to pornography. Social Forces, 75, 957-982.

Sherkat, D. E., \& Ellison, C. G. (1999). Recent developments and current controversies in the sociology of religion. Annual Review of Sociology, 25, 363-94.

Silk, M. (Ed.) (2000). Religion and American politics: The 2000 election in context. Hartford, CT: The Pew Program on Religion and the News Media Center for the Study of Religion in Public Life.

Singham, M. (2000). The science and religion wars. Phi Delta Kappan, 81, 424-432.

Sizer, T. R. (1992). Horace's school: Redesigning the American high school. New York: Houghton Mifflin Company.

Smith, C. (2000). Christian America? What evangelicals really want. Berkley: University of California Press.

Smith, D. C., Neff, D. O., \& Nemes, J. (1999). Assessing race and gender performance differences in KIRIS accountability cycle 2 result (Kentucky Department of Education Paper Series). Frankfort: Kentucky Department of Education..

Smith, M., \& Martin, S. S. (1997). Parent involvement in a program for rural youth atrisk: An exploratory study. The Rural Educator, 19(2), 15-19.

Smith, G. A. (2002). Place-based education: Learning to be where we are. Phi Delta Kappan, 83, 584-594.

Smolowe, J. (1993, November 1). Education: Crusade for the classroom. Time, 34-35. 
Society for the Scientific Study of Religion. (2000, October). Religion and

Transnationalism: Challenges of the 21st century. And religious research.

Gender, religious organization and practice [Brochure]. Houston, TX.

Spiegel, L. A. (1997). Using adolescent literature to develop student pride in rural living. The Rural Educator, 19(1), 13-18.

Sports. (2003, February 6). The Journal-Enterprise (Providence, KY), pp. 15-20.

Stark, R. (1999). A theory of revelations. Journal for the Scientific Study of Religion, $38,287-307$.

Stark, R., \& Glock, C. Y. (1968). American piety: The nature of religious commitment. Berkeley: University of California Press.

Steinberg, L. (1996). Beyond the classroom: Why school reform has failed and what parents need to do. New York: Simon \& Schuster.

Stevens, J. P. (2002). Applied multivariate statistics for the social sciences (4th ed.). Mahwah, NJ: Lawrence Erlbaum Associates.

Strange, M. (2002). New report examines perceptions of rural America. Rural Policy Matters: A Newsletter of Rural School \& Community Action, 4(1), 2.

Strange, M. (2003). A microscopic drop in the research bucket: The dismal state of rural research. Rural Policy Matters: A Newsletter of Rural School \& Community Action, 5(6), 1.

Strange, M. (2004). Rural education wages lag. Rural Policy Matters: A Newsletter of Rural School \& Community Action, 6(8), 1.

Strong families, strong schools. (1996, September). Community Update. United States Department of Education.

Strong, R., Silver, H. F., \& Robinson, A. (1995). What do students want: And what really motivates them? Educational Leadership, 53(1), 8-12.

Summit highlights religious groups' support of education. (1997). Community Update. United States Department of Education.

Tabachnick, B. G., \& Fidell, L. S. (1996). Using multivariate statistics (3rd ed.). New York: HarperCollins College Publishers.

Tennies, R. H. (1986). A parent involvement program including communication to parents integrated with a parent education program and its effect on academic achievement, classroom conduct, study habits, and attitudes. Community 
Theobald, P., \& Nachtigal, P. (1995). Culture, community, and the promise of rural education. Phi Delta Kappan, 77, 132-135.

Thompson, E. H., \& Remmes, K. R. (2002). Does masculinity thwart being religious? An examination of older men's religiousness. Journal for the Scientific Study of Religion, 41, 521-532.

Thorkildsen, R., \& Stein, M. R. S. (1998). Is parent involvement related to student achievement? Exploring the evidence. News, Notes, and Quotes, 43(2), 17-20.

Thurber, F. (1997). Art education and rural schools: Reflections from the field. The Rural Educator, 18(2), 30-34.

Tucker, E (1994). Foundations of educational policy. Unpublished manuscript, University of Louisville.

Tucker, E (1995). Rural policy: Rural-Americans as minority. Unpublished Manuscript, University of Louisville.

Useem, E. L. (1991). Student selection into course sequences in mathematics: The impact of parental involvement and school policies. Journal of Research on Adolescence, 1, 231-250.

Verhoog, H. (1995). The oppressed tradition of caring objectively in Western culture. In Agriculture and spirituality: Inter(agri)cultural dialogue: Essays from the Crossroads Conference at Wageningen Agricultural University (pp. 43-49). Utrecht, the Netherlands: International Books.

Verkleij, F. (1995). Spirituality and ecological agriculture. In Agriculture and spirituality: Inter(agri)cultural dialogue: Essays from the Crossroads Conference at Wageningen Agricultural University (pp. 59-63). Utrecht, the Netherlands: International Books.

Walker, H., \& Sylwester, R. (1991). Where is school along the path to prison? Educational Leadership, 48(1), 14-16.

Watt, D. H. (2002). Bible-carrying Christians: Conservative Protestants and social power. Oxford, NY: Oxford University Press.

White, L, R. (1982). The relation between socioeconomic status and academic achievement. Psychological Bulletin, 91, 461-481.

Wilson, C. R., \& Ferris, W. (1989). Encyclopedia of southern culture. Chapel Hill: University of North Carolina Press. 
Wingfield, M. (1994, May 3). Literacy missions volunteers needed. Western Recorder, p. 1.

Witte, R. (1995). Spirituality and agriculture: What difference would it make? In Agriculture and spirituality: Inter(agri)cultural dialogue: Essays from the Crossroads Conference at Wageningen Agricultural University (pp. 50-58). Utrecht, the Netherlands: International Books.

Wlodkowski, R. J., \& Ginsberg, M. B. (1995). A framework for culturally responsive teaching. Educational Leadership, 53(1), 17-21.

Wuthnow, R. (1973). Religious commitment and conservatism: In search of an elusive relationship. In C. Y. Glock (Ed.), Religion in sociological perspective: Essays in the empirical study of religion (pp. 117-132). Elmont, CA: Wadsworth Publishing Co.

Yin, R. K., (1989). Case study research: Design and methods (Rev. ed.). Newbury Park, CA: Sage Publications.

Youth. (1997, September 2). Western Recorder, p. 6.

Zetterberg, H. L. (1966). Sociology in a new key. New York: Bedminster Press.

Zweers, W. (1995). Ecological spirituality as point of departure for an inter-cultural dialogue. In Agriculture and spirituality: Inter(agri)cultural dialogue: Essays from the Crossroads Conference at Wageningen Agricultural University (pp. 6483). Utrecht, the Netherlands: International Books. 
APPENDIX A

PARENT-TEICIIER CONFERENCE

October 21. 1999

Guest Register

Teacher

Parent

Student

1. Shirlene Pamav

- Samie Couse Rachel house

3. Ade Whotledar Emify Whitledo

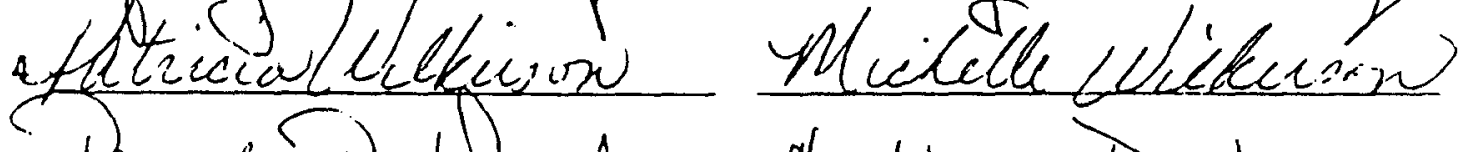

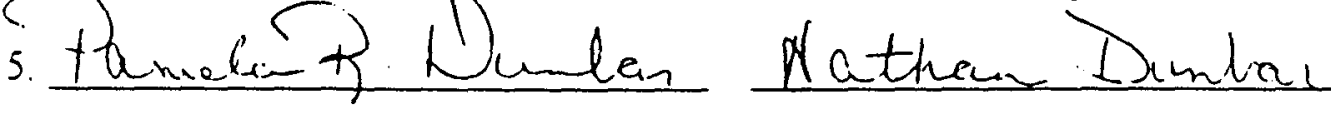

6. OQ Puen , phay

Jort Wheraininy

7. sette livetemon Amond's Nally

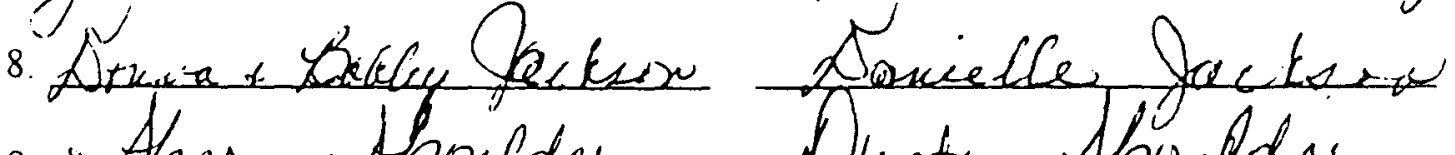

- Atere thoreder Dusty thinedere

10. Trinly Swansox Brittany) Swanior

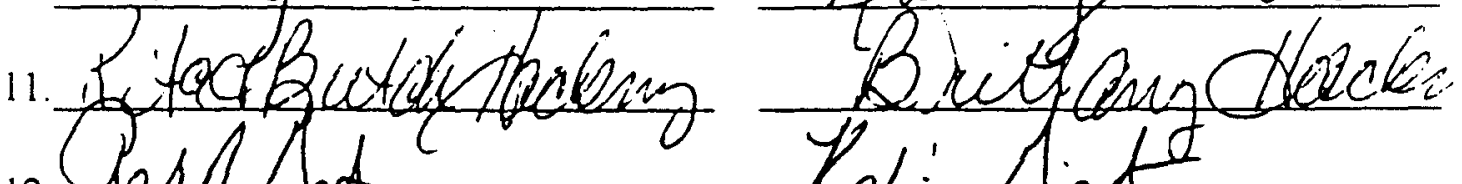

12. pafA.t.bot Kebie taof

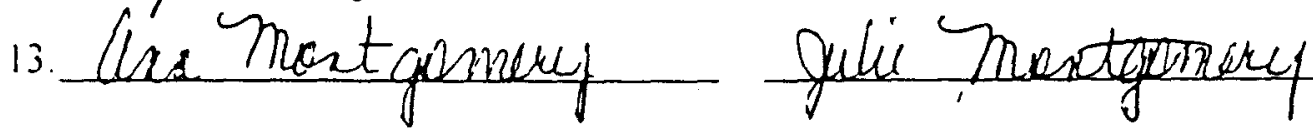

14.

15.

16

313 


\title{
School Report Card
}

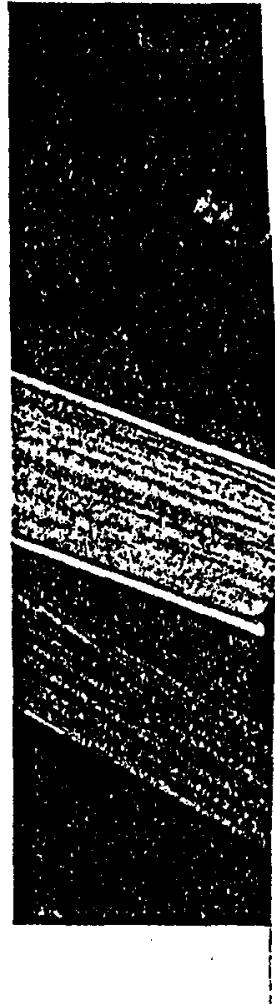

Dear Parents/Guardians -

This report provides information about how well our school is doing - where it is succeeding and where there is room for improvenent. While it cannot tell everything about our performance, the report is a good starting point for discussions with our teachers, administrators, school board members and school council members.

\author{
$1999-2000$ \\ Dixon Elementary \\ Michael Crouse, Principal \\ 277 State Route 1340 \\ Dixon, KY 42409 \\ Phone: (270)-639-9080 \\ Fax: (270)-639-6823 \\ Email: mcrouse2@webster.k12.ky.us
}

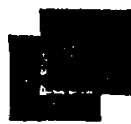

\section{About Our School}

Dixon School is a unique combination of PS $-8^{\text {th }}$ grade students. Our mission is to meet the unique needs of every child with the assistance of parents in our community. Dixon students represent the diversity of our community which includes our heritage of agriculture, mining and industry, and recently increased appreciation for the arts.

Please feel welcome to stop by our school to leam more specific information about our School Report Card.

Total Enrollment (1998-99): 


\section{How Our Students Perform}

Dixon

Kentucky uses the Commonwealth Accountability Testing System (CATS) to hold schools accountable for student progress. CATS has three parts: The Kentucky Core Content Tests, the national Comprehensive Test of Basic Skills (CTBS-5), und other information about the school's performance, such as attendance and retention rates. Together, these three elements make up a school's CATS Performance Score for every two-year period.

\section{Kentucky Core Content Tests}

These tests measure the ability of each student to use knowledge in each subject. The tests are graded on a scale of 0 to 140 points. The state goal for all schools is 100 by 2014. These scores are for tests given in April 1999.

\begin{tabular}{l|l|l|l|}
\multicolumn{1}{c|}{$4^{\text {th }}$ Grade } \\
\cline { 2 - 4 } & Reading & Science & Writing \\
\hline $\begin{array}{l}\text { Our School } \\
\text { District } \\
\text { State }\end{array}$ & 69 & 52 & 48 \\
\cline { 2 - 4 } & 75 & 53 & 45 \\
\hline 76 & 55 & 52 \\
\hline
\end{tabular}

\begin{tabular}{|l|l|l|l|}
\hline Math & Social Studies & Arts \& Humanities & $\begin{array}{c}\text { Practical Living/Vocational } \\
\text { Studies }\end{array}$ \\
\hline 60 & 49 & 22 & 38 \\
\hline 65 & 54 & 29 & 38 \\
\hline 64 & 57 & 31 & 45 \\
\hline
\end{tabular}

\section{National Basic Skills Test}

The national basic skills test that is used in Kentucky, the CTBS-5, measures the basic skills of our students while allowing us to compare our performance to the performance of students throughout the country. These scores are reported in percentiles. A percentile shows the percentage of students who fell below a particular score on the test. For example, a percentile of 60 would show that the average student in our school scored equal to or better than 60 percent of all the students who took the test. These scores are for tests given in April 1999.

\begin{tabular}{|c|c|c|c|}
\hline & $\begin{array}{l}\text { Exiting Primary (P4 or Grade 3) } \\
\text { Reading }\end{array}$ & $\begin{array}{c}\text { Exiting Primary (P4 or Grade 3) } \\
\text { Language Arts }\end{array}$ & $\begin{array}{c}\text { Exiting Primary (P4 or Grade 3) } \\
\text { Math }\end{array}$ \\
\hline \multirow{4}{*}{$\begin{array}{l}\text { Our School } \\
\text { District } \\
\text { State } \\
\text { Nation }\end{array}$} & $71 \%$ tile & $59 \%$ tile & 57\%tile \\
\hline & $57 \%$ tile & $56 \%$ tile & $53 \%$ tile \\
\hline & 51\%tile & $50 \%$ tile & S1\%tile \\
\hline & $50 \%$ tile & $50 \%$ tile & $50 \%$ tile \\
\hline
\end{tabular}

\section{Other Measures}

Other measures of school performance are the degree to which our school succeeds in getting students to come to school, and the degree to which our school is preparing students to be successful in the next grade the next year. The retention rate reflects the percent of students who were not academically ready to go onto the next grade the next year and had to repeat the content of a grade for another year. These data are collected from the school year $1997-1998$.

Our School District State
Average Daily Attendance

Retention Rate (4 $4^{\text {th }}$ and $5^{\text {th }}$ Grades only)

\begin{tabular}{|l|l|}
\hline \multicolumn{1}{|c|}{ Average Daily Attendance } & $0 \%$ \\
\hline $94 \%$ & $0 \%$ \\
\hline $94 \%$ & $3 \%$ \\
\hline
\end{tabular}




\section{acurricular Activities}

And

Axpards ar

Am, Beta Club, Speech Team, Math Team, Band, Chorus, Cross Country, Track, Football, Basketball

Stidentrontieved state level recognition in Academics, Speech, Cross Country, Track, and Chons.

Th f Theam received $3^{\text {th }}$ place at the Regional Math Campetitions.

\section{What We Are Doing to Improve}

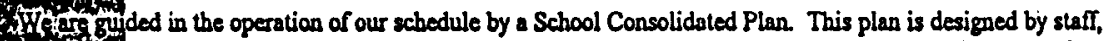
1) parents and members of the community, reviewing our past performance in academics and social responsiveness. 6) f. Performances are used as a benchmark to which we compare future accomplishments. We certainly look for - Hoy to mprove our school community as we seck to mointain and nurture the good things we are already doing

\section{XX To Get More Information}

Call far fax or write our principal at the number and address listed on the front page of the report card and ask for the
Wxpanded school Report" Call your School-Based Decision Making (SBDM) Council members:

\begin{tabular}{|c|c|c|c|}
\hline $\begin{array}{l}\text { Member's Name } \\
\text { Michael D. Crouse }\end{array}$ & $\begin{array}{l}\text { Phone } \\
639-5609\end{array}$ & Member's Name & Phone \\
\hline 4 y Susie Collins & $830-6086$ & & \\
\hline TTerri Lovan & $664-6493$ & & \\
\hline Hoskins & $826-3705$ & & \\
\hline Aly thithyn Alvey & $639-0090$ & & \\
\hline (4) & $667-5868$ & & \\
\hline
\end{tabular}




\section{Our CATS Performance}

Starting in the year 2001, every school will have its own growth chart. Here, you will be able to track our school's scademic improvement yearly.

Our School's customized growth chart will be placed here in the year 2001

\section{Teacher Qualifications}

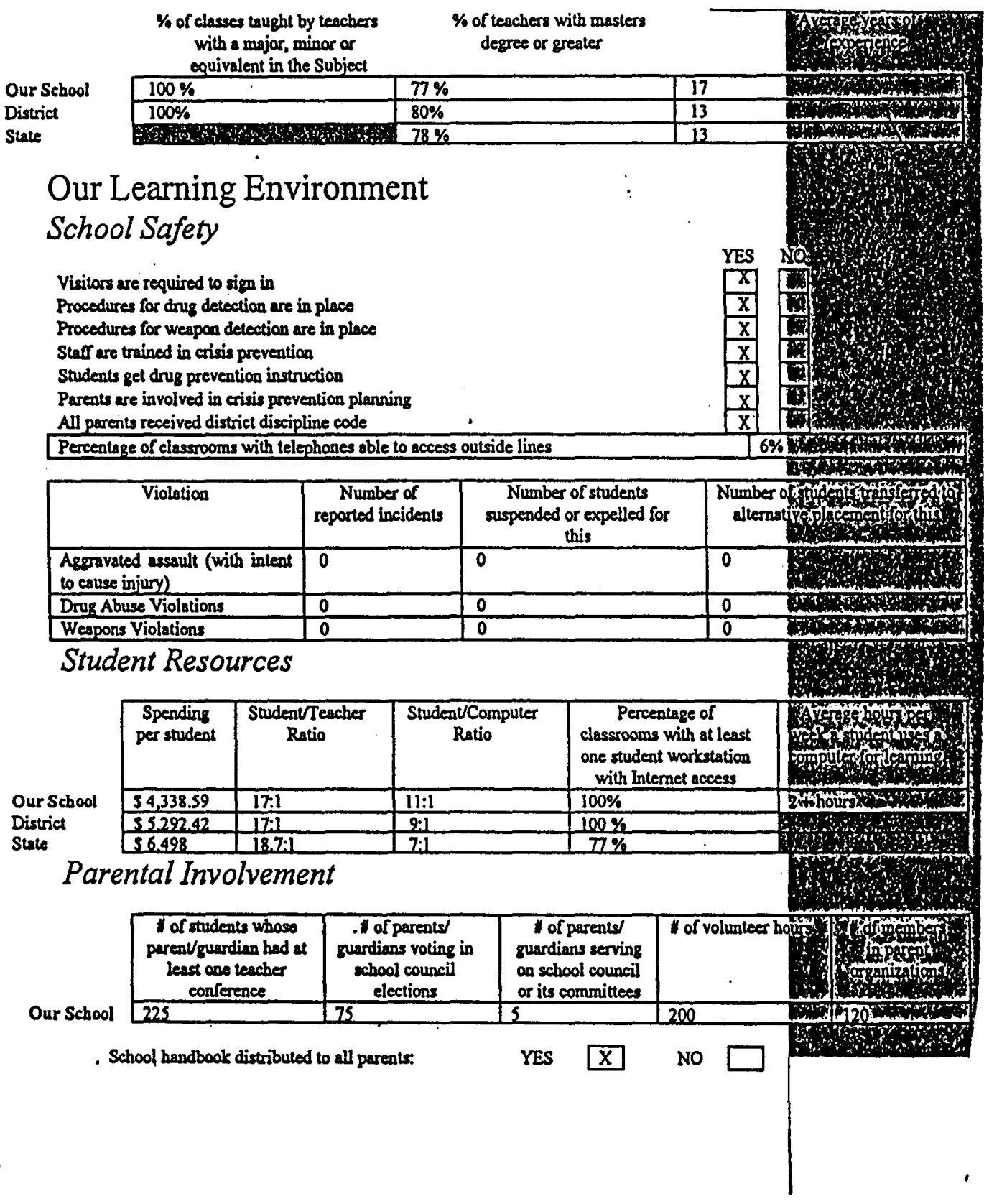




\title{
APPENDIX C
}

\author{
1 \\ PETITION \\ TO REPEAL KERNOAE \\ ANO \\ RESCINO AHENOMENTS
}

WE THE PEORLE OF THE COMMONWEALTH OF KENTUCKY DO AETTION THE GENERAL ASSEMBLY OF ELECTEO REPRESENTA TNES ANO ELECTED SENATORS AND THE GOVERNOR OF THE COMHOMWEALTH OF XENTUCKY TO REPEAL THE XEVTUCXY EDUCA TION REFORM ACT ALSO KNOWN AS KERA.

We ine Arople of the Commonwe dth of Kenlucky ere concemed citizans end perma. We ere grieved by the trpe of education relem mendeled by che Kenrucky Educeoion Reform AeC Wo ere grioved by die aroirude chenges in our children. by the sugnmicant chenges in the chereom, oy the experinente nerur of the rotorm, end by the deremphexis

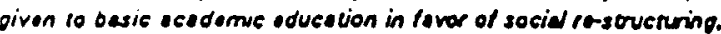

We che Prople of the Commonwedth of Kentuck thersater roforrod to as "Wh" I recognize che system or mpe of - ducacion reform driving KERA and We objece to the inclusion of enY oducedion rotom that b Lased on, bortomed, or driven

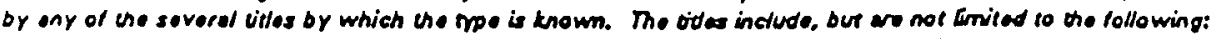
PERFORMANCERASEO EOUCATION, OUTCOMEISJ-RASEO EDUCA HON, OUTCOMESALUNEO EDUCA TION, NEW AMEAICAN SCHOOLS, SCHOOLS FOR THE 21 ST CENTURY, VALUES CLAAIFCA NON, MASTERY LEARNINO, GOALS

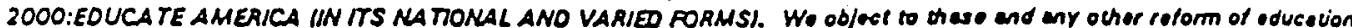
chet wes, as its driving principle, the phibsophlas of the proponeno of THE NEW WORLO OROER, SOCLAL

RESTRUCTURING, MULTICULTURALSH, WOALO CTTEENSHIP. THE NEW AOE MOVEMENT, and similar philasophiss UAt DIOMOI - RESTRUCTURINO OF OUA SOCIETY IN ECONOMY, ROUTICS, RELGION, ANO FAMILY SOVEREIGNTY.

We recognize the entempt to implement the forommoioned phibephlas a RESTRUCTURINO TOOLS appliod chrough KENTUCKY EOUCA TION REFORM ACT and twough other moese in Kentuatr and ather States in the Union embrecing part or all of the principles of this philasophy of aducedion reform summariad in ohis documene as OUTCOMES. BASEO EOUCATION.

Wo PETITION Che General Assembly and Governor to RESTORE the eCquisioion of ACAOEHIC KNOWLOGE and SKILLS as the primer puppose of educabion.

Wo PETITION the AEHOVAL of anY and allogislevian thet hinders or mil hinder che implementebion of Acedemit

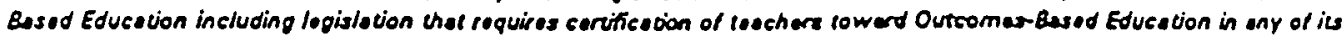
several forms.

Wo PETITION the General Assembly and Governor to RESTORE the Office of Superintendent of Fublic hutruction w en ELECTEO OFACE by introduction of such legislobion as wouddroped the removal of thet Office by Amendment.

Wo PETITION the Generd Assumbly and the Governor to REPEAl the Amendment ormeing che Governor power to sppoint a Commissioner of Educetion not alected by the Praple end to immediatety devolve the offtee of Cammiesioner of Educaition and RESTORE the Fower of Choice of Educaoin to the Proplat.

W. RESERVE THE RIGHT TO PRNACY, as indfiduab, as panna, as studeno, and a fanilles.

We RESERVE THE RIGHT to dLAllow experimented educeoion programs and/or Deschobgice lesting endlor oretment of our children by oovernment or educesion as tablithmente

We RESERVE. THE RIOHT to educete our chidden as permts, es temillas, and as toc.V communities eccording to the dicrectes of Conscionce.

We RESERVE THE RIGHT to bed school boerd olecoions wiotout mandete tram the Stafe or Fiderd Government

WO RESERVE THE RIGHT TO RESTORE End MAINTAW E EN REPAESEMTATVE OEHOCRACY h the Commonweelth of Kentucky and as Citcens of the United Statas of Americe, to zuppart end defend the Canstituton of use United Slefes of Americe end The Constitution of the Commonwedch of Kentuck

We the Poople of the Commonwealth of Kentucky do present ents AETTION and iffix our nemes in the hope of ussurance that our Eected Representecives will comph in like mind

I am in full agreement with the Petition to Repeal KERA/OBE and Rescind Amendments. I afflrm that I have read or heard the contents of the petition in its entirety. My signature affirms my support of the Petition.

COMMENTS (IF DESIRED): 


\section{APPENDIX D}

\section{EDUCATING OUR CHILDREN}

\section{THIS IS A MAJOR EMERGENCY}

We believe Kentucky Citizens armed with the Truths about Kentucky's Education System will rise up to the aid of a Precious Child's Education and voice their concerns to our elected officials by demanding change for the betterment of Children and Teachers.

EDUCATION IS ON THE BRINK OF DISASTER IN KENTUCKY. THE KENTUCKY EDUCATION REFORM ACT (KERA) MUST BE REPEALED FOR THE FOLLOWING REASONS:

1. KERA has been presented in difficult to understand language that is an intentional plan to misinform parents and voters. Kentucky legislators no longer in office have admitted they did not understand it when they voted for it. Your CURRENT State legislators admit that they don't understand it either...therefore they will not oppose it.

2. KERA is using Kentucky students as guinea pigs in a major social experiment that is failing miserably.

3. KERA has no objective standards of achievement. If it is allowed to continue, your children will be uneducated and millions of dollars will be wasted.

4. KERA is marketed as "el iminating student failure" but in reality is designed to remediate children until they succumb to the social outcomes that are parallel with the United Nations World Curriculum.

KERA will produce followers not leaders. The outcome requirements are designed to create an economical "workforce" that doesn't argue, question, or think for itself but will be efficient at reporting and data collection.

6. KFRA is intended to shape all children's values to conform to those planned by the state. PARENT'S VALUES DON'T COUNT. Do you want your children remediated over and over with a "one world value system"?

7. KERA is networking computer systems now to track your child and the entire family. Privacy is no longer your right. Non-conforming households could be labeled as having children "at risk" and the state could take control to mandate how you raise your children. You may be considered to have an "at risk" child if you are overweight. smoke. on government assistance. if your child missed breakfast. or any other ridiculous reason the state may decicle to use.

8. KERA is intentionally not using intense phonics to teach your child to read. Intense phonics is the ONLY method of teaching reading that has documentation to PROVE it produces good readers.

9. KERA lies when it says you have local control in the school. What you do locally MUST meet STATE and FEDERAL directives.

10. KERA prevents teachers from opposing or objecting to the reforms. Most teachers are opposed to KERA but are afraid to voice it for fear of losing their job. Is this Kentucky or Communist China? 


\section{WHAT CAN YOU DO?}

1. CALL OR WRITE YOUR STATE REPRESENTATIVES TOOAY. DEMANO THAT KERA BE REPEALED. IT'S YOUR HONEY THEY'RE SPENDING.

Don't let Kentucky children be used in this experiment that was designed and directed by behavioral psychologists, not educators. These people equate your children and grandchildren with rats in a maze. You've heard of Pavlov's slobbering dogs. Now find out the truth about KERA and OUTCOME BASED EDUCATION.

2. CALL OR WRITE YOUR SENATORS AND REPRESENTATIVES IN WASHINGTON, D.C. AND TELL THEM TO VOTE "NO" ON "H.R. 1804. GOALS 2000: EDUCATE AMERICA ACT" AS IT IS HORE OF THE SAME PROBLEM AS IN KENTUCKY, ONLY WORSE.

KERA is the reform act that implements OUTCOHE BASED EDUCATION in Kentucky. "GOALS 2000" affects us nationally and will reach much farther into our lives to control YouR children and grandchildren. OUTCOHE BASED EDUCATION is in different stages of implementation across oul country and is aimost iuentical in goais and outcomes in each state. "GoALS $2000^{\circ}$ will "cement it in" and speed up the implementation process, casting a dark cloud over the future of your children and grandchildren.

3. GET INYOLVED LOCALLY.

Find out what is going on in your child's classroom. You will be surprised to find out what is and what is not being taught. Voice your opposition to OBE proponents. Attend school meetings, Board of Education meetings and the local T.E.A.C.H. meetings. Inform others about OUTCOME BASED EDUCATION. People are winning this battle in other states because they are organizing and letting the politicians know how they will vote.

\section{T. E. A. C. H.}

The name of the group is T.E.A.C.H. which stands for Taking Education and Children Higher.

We are a group of citizens made up of parents, grandparents. educators and others concerned with the education process of the Commonwealth of Kentucky and more specifically Henderson County. T.E.A.C.H. is committed to taking education and children to a higher level. Having this commitment: Our purpose is to educate citizens to those aspects of the Kentucky Education Reform Act of 1990 which we believe are not to the benefit of good education and to advocate change necessary for the betterment of education in Henderson and the Commonwealth.

\section{$\frac{\text { FOR HORE INFORMATION }}{\text { (CHECK AND MAIL) }}$} I would like more information on "OUTCOME BASED EDUCATION" in Kentucky - KERA and the
United States - Goals 2000 . Please notify me of your next meeting. I would like to hear more about outcome Based
Education and how it affects children. 


\section{APPENDIX E}

$>$ THE 2000 FEDERAL CENSUS FOR THE SOUTH

$>$

$>$ Last name:

$>$

$>$ First name: (Check appropriate box)

$>\quad($ Billy-Bob

$>($ Billy-Joe

$>\quad$ Billy-Ray

$>($ Billy-Sue

$>()$ Billy-Mae

$>$ ( Billy-Jack

$>$

$>$ What does everyone call you?

$>\bigcup$ Booger

$>\circlearrowleft$ Bubba

$>\circlearrowleft$ Junior

$>\circlearrowleft$ Sissy

$>\quad$ Other

$>$

$>$ Age:

(if unsure, guess)

$>$

$>$ Sex:

M $\mathrm{F}$ Not sure

$>$

$>$ Shoe Size: Left Right

$>$

$>$ Occupation: (Check appropriate box)

$>\bigcup$ Farmer

$>($ Mechanic

$>\quad \bigcup$ Hair Dresser

$>\bigcup$ Unemployed

$>\bigcup$ Dirty Politician 


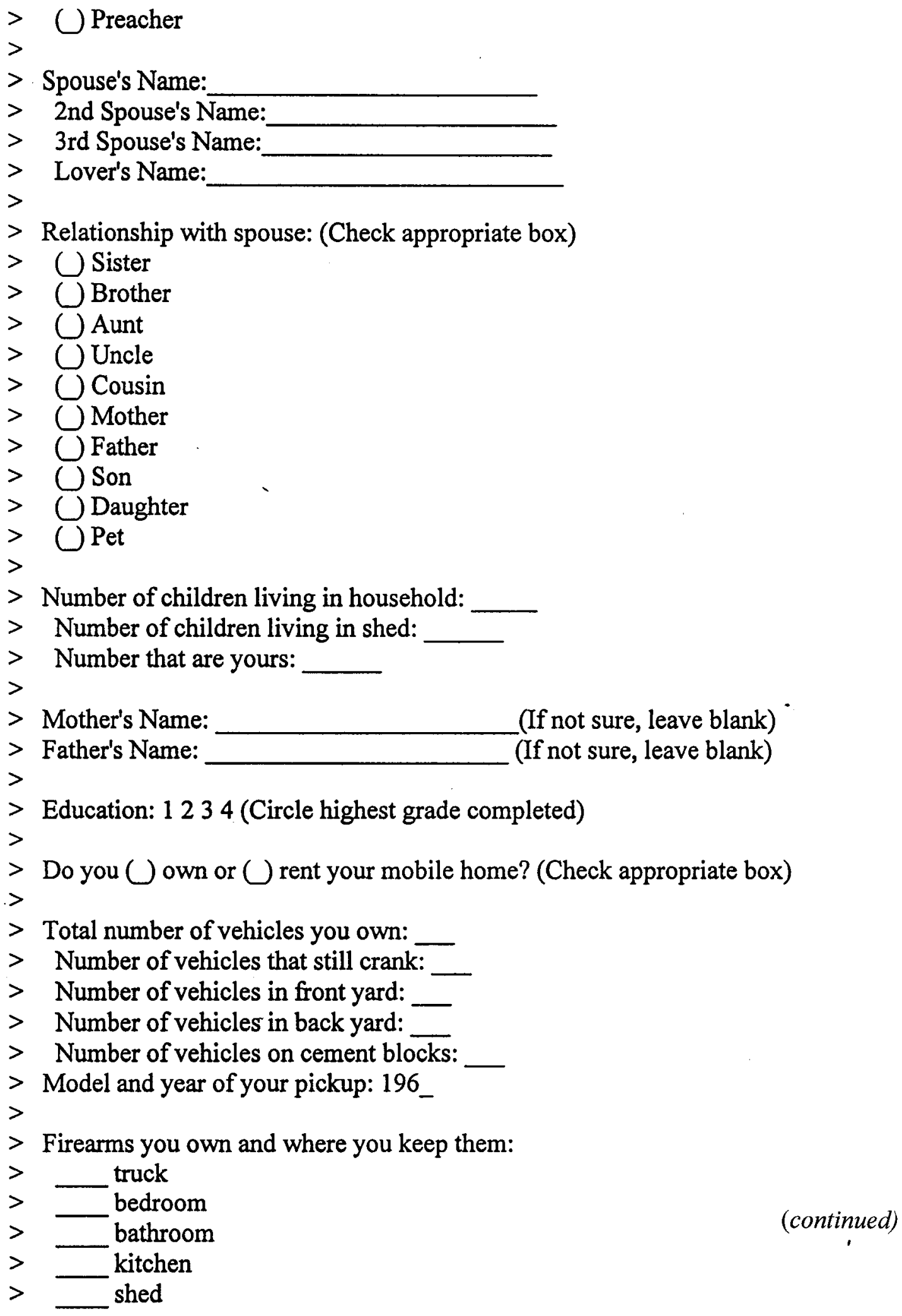




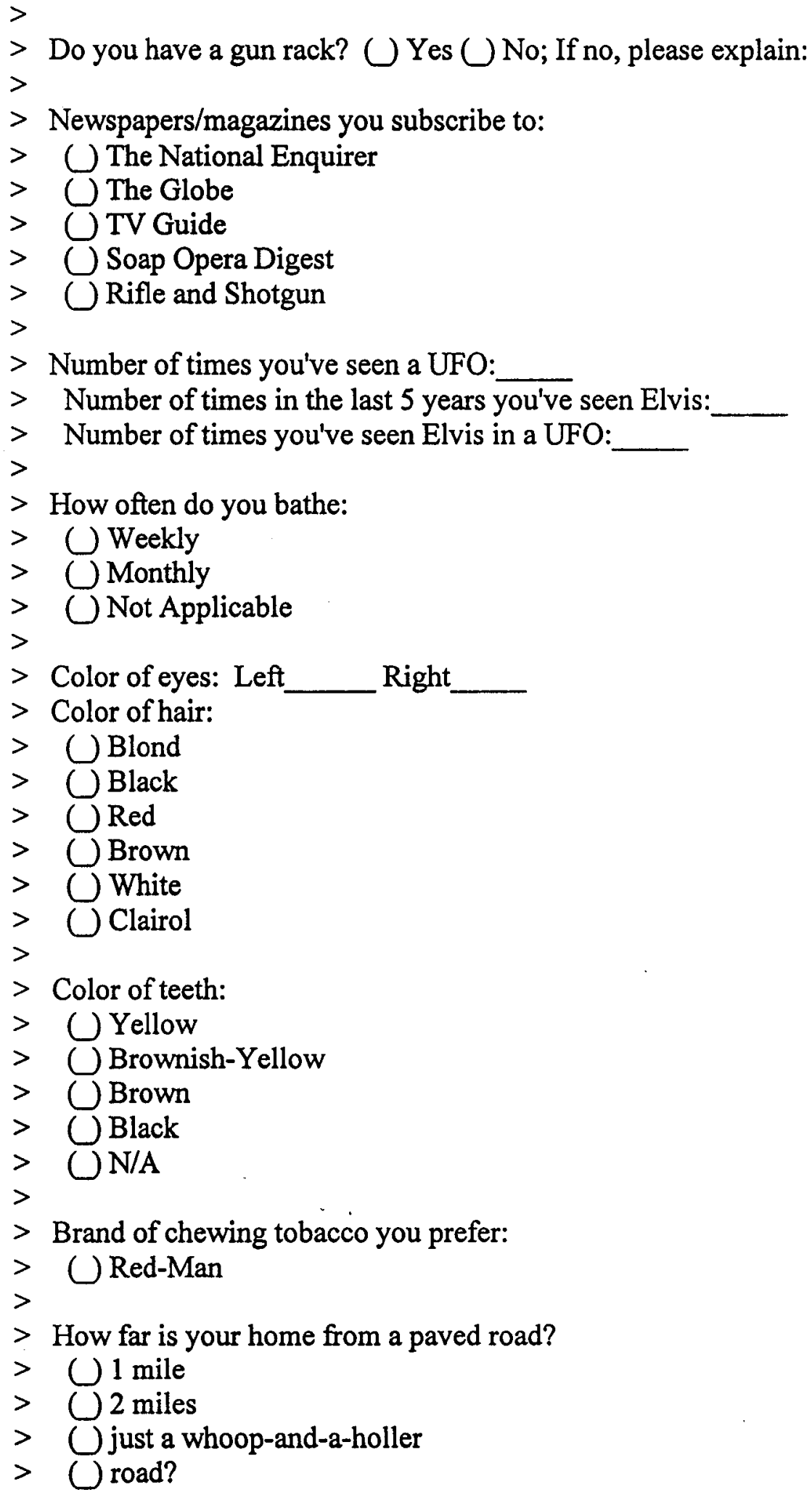




\section{CHARACTERISTICS OF RURAL GIFTED STUDENTS}

Advantaged Rural Gifted Students middle class children whose behaviors reflect the traditional values of the dominant culture
Disadvantaged Rural Gifted Students economically disadvantaged and/or geographically isolated children whose behaviors reflect traditional Anglo-

Appalachian cultural values
1. Speak standard English

2. Are verbal and have good communication skills

3. Are active particpants in classroom activities

4. Perform tasks within time limitations

5. Complete classroom assignments and homework

6. Perform well on standardized tests

7. Perform well in all subjects

8. Produce written work in proper grammatical form with good spelling and legible handwriting

9. Demonstrate their strengths within the academic classroom

10. Usually perform equally well on verbal and non-verbal tests.
1. Speak a non-standard regional dialect

2. Are less verbal in oral communication skills

3. Tend to be passive participants in classroom activities

4. Are relatively unaffected by time pressures; work slowly but meticulously

5. Are likely to be lax in completing assignments and homework

6. Are not likely to perform well on standardized tests

7. May show exceptional ability in one subject and average to below average in others

8. Have written products that may be of high quality in content but of poor quality in grammatical form, spelling and handwriting

9. More likely to demonstrate their strengths outside the classroom, i.e., auto and tractor repair, knowledge specific to their rural environment, creativity related to $4-\mathrm{H}$ projects, talent in music and the performing arts

10. Are likely to perform better on nonverbal than verbal tests.

O Projar S.P.R.N.G., Inciane U.iversity 


\title{
APPENDIX G
}

\author{
$>$ Sent: Tuesday, February 19, 2002 9:12 AM \\ $>>$ \\ $>>>$ Dear Consumers: \\ $>>>$ It has come to our attention that a few copies of the WINDOWS 98 \\ $>>>$ SOUTHERN EDITION may have accidentally been shipped outside the South.
}

$>>>$

$>>>$ If you have one of these, you may need some help understanding the

$>>>$ commands. The Southern edition may be recognized by the unique opening

$>>>$ screen.

$>>>$

$>>>$ It reads: WINDERS 98 , with a background picture of General Robert $E$.

$>$ Lee

$>>>$ superimposed on a Confederate flag. It is shipped with a Dukes of Hazzard

$>>>$ screen saver.

$>>>$

$>>>$ Please also note:

$>>>$ The Recycle Bin is labeled "Outhouse"

$>>>$ My Computer is called "This Dern Contraption"

$>>>$ Dial up Networking is called "Good Ol' Boys"

$>>>$ Control Panel is known as the "The Dashboard"

$>>>$ Hard Drive is referred to as " 4 Wheel Drive"

$>>>$ Floppies are "them little ol plastic disc thangs"

$>>>$ Instead of an error message a "garbage bag and roll of duct tape" pops

$>>$ up.

$>>>$

$>>>$ CHANGES IN TERMINOLOGY IN SOUTHERN EDITION:

$>>>$ OK......... ats aw-right

$>>>$ Cancel ....... stopdat

$>>>$ Reset........ try er agin

$>>>$ Yes......... yep

$>>>$ No ......... noop

$>>>$ Find ........ hunt fer it

$>>>$ Go to. . . . . . . over yonder

$>>>$ Back ....... back yonder

$>>>$ Help . . . . . . hep me out here

$>>>$ Stop ......... kwitit

$>>>$ Start . . . . . . . crank er up

$>>>$ Settings ...... settins

$>>>$ Programs ..... stuff at duz stuff

$>>$ Documents ... stuff ah done did

$>>>$

$>>$ Also note that SOUTHERN EDITION does not recognize capital letters or

$>>>$ punctuation marks.

$>>>$

$>>$ some programs that are exclusive to Winders 98 :

$>>>$ Tiperiter . . . . . . . A word processing program

$>>>$ colerin book . . . . . a graphics program

$>>>$ cyferin mersheen. calculator

$>>>$ outhouse paper . . notepad

$>>>$ iner-net ........ Microsoft Explorer 4.0

$>>>$ pichers . . . . . . . . A graphics viewer

$>>$ We regret any inconvenience it may have caused if you received a copy or $>>>$ the SOUTHERN EDITION. You may return it to Microsoft for a replacement $>>>$ version.

$>>>$

$>>>$ I hope this helps all $y^{\prime}$ all!

$>>$ Billy Bob Gates

... 


\section{APPENDIX H}

Sent: Tuesday, May 08, 2001 6:30 PM

$>>>$ Things a True Southerner Knows:

$>>>$

$>>>$ The difference between a hissie fit and a

$>>>$ conniption fit.

$>>>$ Pretty much how many fish make up a mess.

$>>>>$

$>>>$ What general direction cattywumpus is.

$>>>>$

$>>>$ That "gimme sugar" don't mean pass the sugar.

$>>>$

$>>>>$ When somebody's "fixin" to do something, it won't be long.

$>>>$

$>>>$ The difference between Yankees and damn Yankees.

$>>>>$

$>>>$ How good a cold grape Nehi and cheese crackers are at a country

$>>>$ store.

$>>>>$

$>>>$ Knows what "Well I Suwannee !!" means.

$>>>$

$>>>$ Ain't nobody's biscuits like Grandma's biscuits !!

$>>>$

$>>>A$ good dog is worth its weight in gold.

$>>>$

$>>>$ Real gravy don't come from the store.

$>>>$

$>>>$ When "by and by" is.

$>>>$

$>>>$ How to handle their "pot likker".

$>>>$

$>>>>$ The difference between "pert' near" and "a

$>>>$ right far piece".

$>>>$

$>>>$ The differences between a redneck, a good ol'

$>>>$ boy, and trailer trash.

$>>>$

$>>>>$ Never to go snipe hunting twice.

$>>>$

$>>>$ At one point learned what happens when you swallow tobacco juice. 
$>>>>$ Never to assume that the other car with the

$>>>>$ flashing turn signal is actually going to make a turn.

$>>>$

$>>>>$ You may wear long sleeves, but you should

$>>>>$ always roll 'em up past the elbows.

$>>>>$

$>>>>$ You should never loan your tools, pick-up, or

$>>>>$ gun to nobody.

$>>>>$

$>>>>$ A belt serves a greater purpose than holding Daddy's pants up.

$>>>$

$>>>>$ Rocking chairs and swings are guaranteed stress relievers.

$>>>>$

$>>>>$ Rocking chairs and swings with an old person in them are history $>>>$ lessons. 


\section{APPENDIX I}

Parent Involvement Interview Selection Matrix

\begin{tabular}{|l|l|}
\hline \multicolumn{1}{|c|}{ Point Values } & Range \\
\hline Presence at $10 / 21 / 99$ P/T Conference $=1$ & $0-1$ \\
\hline Presence on any Field Trip $=2$ & $0-2$ \\
\hline Additional Researcher Field Notes Activities $=1$ & $1-3$ \\
\hline
\end{tabular}

\begin{tabular}{|l|}
\hline $\begin{array}{c}\mathrm{H} / \mathrm{M} / \mathrm{L} \\
\text { Categories }\end{array}$ \\
\hline $\mathrm{H}=5-6$ \\
$\mathrm{M}=3-4$ \\
$\mathrm{~L}=1-2$ \\
\hline
\end{tabular}

Student Achievement Interview Selection Matrix

\begin{tabular}{|lc|}
\hline \multicolumn{1}{|c|}{ Point Values } & Range \\
\hline $75+$ percentile any KIRIS/KCCT Score $=1$ & $0-4$ \\
$80 \%-90 \%$ GPA in any subject $=1$ & $0-4$ \\
& \\
Activities and Leadership listed on Survey & \\
& \\
$1-2$ Activities an/or Leaderships $=1$ & \\
$3-4$ Activities and/or Leaderships $=2$ & \\
$5-6$ Activities and/or Leaderships $=3$ \\
7 or more Activities and/or Leaderships $=4$ & $0-4$ \\
Participation at School based on RFN $=1$ & $1-6$ \\
\hline
\end{tabular}

\begin{tabular}{|l|}
\hline $\begin{array}{c}\mathrm{H} / \mathrm{M} / \mathrm{L} \\
\text { Categories }\end{array}$ \\
$\mathrm{H}=14--18$ \\
$\mathrm{M}=7-13$ \\
$\mathrm{~L}=1-6$ \\
\hline
\end{tabular}




\section{APPENDIX J}

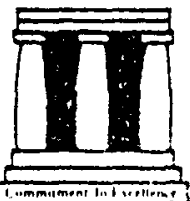

WEBSTER COUNTY SCHOOLS

MICHAEL K. FARRIS, SUPERINTENDENT

Box 420 - Diron, KY 42409 • 270-639-5083

Fax Number 270-639-5086

Lesie Hudson

Chairperson

July 26,2000

Ms. Carolyn Tucker

429 Burnt Mill Road

James Nance

Dixon, KY 42409

Vice Chairman

Dear Ms. Tucker:

Per your request, I have no problem with you conducting surveys and interviews of students and parents of Dixon's $7^{\text {th }}$ and $8^{\text {th }}$ grades on a voluntary basis. I would ask

Lisa Presion Boara Member that this process be conducted in a manner not to interfere with instructional time.

Good luck to you as you strive to achieve your goal.

Luther Willson Board Mernber

Sincerely,

mpickections

Michael K. Farris

Superintendent

Mike Putman

Rachel Yartrough
Director of
Oistrict What Semicas

Webster Ceunty Schools are providers of Equal Educational and Employment opportunities 


\section{APPENDIX K}

\section{ADULT CONSENT FORM}

UofL 555-01/WKU HS 02-053: The Interrelationships of Protestant Religiosity, Rural Values, Parental Involvement, and Student Outcomes in a Small, Rural, Southern, Public, Middle School

I have read the letter of request for my participation in the above study. I understand the purposes of the study to be for the doctoral dissertation of E Carolyn Tucker; to find out how responsive people feel the school system is to family morals and values; and to find out how family beliefs and attitudes relate to how well students do in school.

I realize I am invited, not required, to participate in the survey and/or the interview.

I also understand that there are no foreseeable risks in my participation and that confidentiality will be protected to the extent permitted by law. I do realize that University personnel will see the information as they review this work.

I know that my participation is voluntary and that I may refuse to participate, or to withdraw from participation at any time, without any penalty or loss of benefits. I know that I may speak confidentially to a member of the University of Louisville Human Studies Committee; at 502-852-5188, if I have any questions about my rights as a participant.

My questions about this study have been answered in language I can understand and I know that any future questions I have will be answered in the same manner.

Also, as parent or legal guardian of hereby give my permission for $\mathrm{E}$ Carolyn Tucker to review my child's education records stored at the Webster County Board of Education. I understand that information from achievement test scores is to be used in this doctoral study only. I know that my child's name and scores will never be published. 


\section{PARENTAL CONSENT FORM FOR CHILD SURVEY PARTICIPATION}

UofL 555-01/WKU HS 02-053: The Interrelationships of Protestant Religiosity, Rural Values, Parental Involvement, and Student Outcomes in a Small, Rural, Southern, Public, Middle School

I have read the letter of request for my child's participation in the above study. I understand the purposes of the study to be for the doctoral dissertation of E Carolyn Tucker; to find out how responsive people feel the school system is to family morals and values; and to find out how family beliefs and attitudes relate to how well students do in school.

I realize my child is invited, not required, to participate in the survey.

I also understand that there are no foreseeable risks in my child's participation and that confidentiality will be protected to the extent permitted by law. I do realize that University personnel will see the information as they review this work.

I know that my child's participation is voluntary and that I may refuse to allow him/her to participate without any penalty or loss of benefits. I know that I may speak confidentially to a member of the University of Louisville Human Studies Committee, at 502-852-5188, if I have any questions about my child's rights as a participant and that I may withdraw my child from the study at any time without penalty of any kind.

My questions about this study have been answered in language I can understand and I know that any future questions. I have will be answered in the same manner.

Child's Name

Parent Signature

Date 


\section{APPENDIX M}

\section{PARENTAL CONSENT FORM FOR CHILD INTERVIEW}

\section{UofL 555-01/WKU HS 02-053: The Interrelationships of Protestant}

Religiosity, Rural Values, Parental Involvement, and Student Outcomes in a Small, Rural, Southern, Public, Middle School

I have read the letter of request for my child's participation in the above study. I understand the purposes of the study to be for the doctoral dissertation of E Carolyn Tucker; to find out how responsive people feel the school system is to family morals and values; and to find out how family beliefs and attitudes relate to how well students do in school.

I realize my child is invited, not required, to participate in the interview.

I also understand that there are no foreseeable risks in my child's participation and that confidentiality will be protected to the extent permitted by law. I do realize that University personnel will see the information as they review this work.

I know that my child's participation is voluntary and that I may refuse to allow him/her to participate without any penalty or loss of benefits. I know that I may speak confidentially to a member of the University of Louisville Human Studies Committee, at 502-852-5188, if I have any questions about my child's rights as a participant and that I may withdraw my child from the study at any time without penalty of any kind.

My questions about this study have been answered in language I can understand and I know that any future questions I have will be answered in the same manner.

Child's Name

Parent Signature 


\section{APPENDIX N}

[University Letterhead]

[Date]

"The Interrelationships of Protestant Religiosity, Rural Values, Parental Involvement, and Student Outcomes in a Small, Rural, Southern, Public, Middle School"

Dear Parents/Students,

I am seeking your input into a research study I am conducting of parent/student feelings about the Dixon School community. The purpose of this study is to find out how responsive you feel the school system is to family morals and values and how family beliefs and attitudes relate to how well students do in school.

I must complete a study to finish my doctoral work. I want my study to be a useful document for may school community, rather than a simple academic exercise. Please help me to find the information I need to complete my dissertation. To let you know how important it is, I have attached $\mathbf{\$ 1 . 0 0}$ to this letter.

All parents/students of the (2000-2001) $7^{\text {th }}$ and $8^{\text {th }}$ grade classes at Dixon School have been invited to participate in this study. The enclosed survey will take about 15 minutes to complete. Please fill out the parent survey as honestly as you can. Have your $7^{\text {th }}$ or $8^{\text {th }}$ grader from the 2000-2001 school term complete the student survey (no fair sharing answers!). Return the completed surveys in the envelope provided by [completion date]. Later, some parents may be invited to participate in interviews to provide further descriptions of beliefs and attitudes.

There are no foreseeable risks in your participating in my study. But there are some benefits. People who are involved in this study will help identify attitudes and beliefs that influence parent involvement and student outcomes in school. Identifying those beliefs and attitudes could help improve the school's parent involvement programs.

Don't put your name on the survey. I don't want names, but I have coded the survey so that I can connect a parent survey to his/her student survey, and so that I will know who has not returned surveys (for the purpose of follow-up reminders). I will protect your identity as well as I possible can, to the extent permitted by law. University personnel will see the information I collect as they review my work. But, if the study is ever used in any other way, I will change even the name of the school and school district.

Your participation is voluntary and you may refuse to participate without any penalty or loss of benefits that you are entitled to. You have the RIGHT to understand and ask 
questions about this project. You will be able to talk to the Principal Investigator (my Dissertation Advisor), Dr. Stephen Miller, at 502-852-5188, if you have any questions about your rights as a participant. All present questions have been answered in language you can understand and future questions will be treated in the same manner.

Please note PURPOSE, PROCEDURES, POTENTIAL RISKS, and BENEFITS of this study as explained earlier. Although $\mathbf{\$ 1 . 0 0}$ is attached to show how important your response is, there is no obligation for this COMPENSATION. You are INVITED to participate but your decision is VOLUNTARY. CONFIDENTIALITY as discussed is consistent with Federal Human Subjects regulations.

I look forward to hearing from you. If I haven't heard from you in the next few days, I'll call to remind you. If you have any questions, you may call me at 639-5107 or e-mail me at ectucker@kih.net.

Please sign the consent from for YOU and YOUR CHILD.

Thank you so much for your help.

E Carolyn Tucker 


\section{APPENDIX 0}

\section{Parent Involvement}

I A. We know that parents want the best education possible for children. Because Parent Involvement is important for student achievement, it would help us to know how you feel about some of the opportunities for parent involvement at your school.

Please rate the following statements on a scale of 1 to 5 with 1 being "strongly disagree" and 5 being "strongly agree." CIRCLE the number you choose.

1. The school values my participation in my child's Strongly school activities.

2. The staff at school welcomes me when I visit.

$\begin{array}{lllll}\begin{array}{l}\text { Strongly } \\ \text { Disagree } \\ 1\end{array} & 2 & 3 & 4 & \begin{array}{c}\text { Strongly } \\ \text { Agree } \\ 1\end{array} \\ & & & & \end{array}$

3. My child's teacher(s) considers me important in my child's $1 \quad 3 \quad 2 \quad 3 \quad 4 \quad 5$ education.

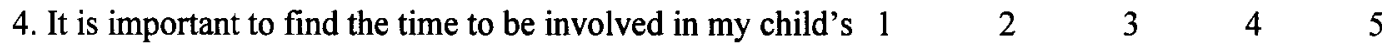
school.

5. My child learns more when I am involved with his/her $\quad \begin{array}{lllllll}1 & 2 & 3 & 4 & 5\end{array}$ schooling.

I B. Please CIRCLE the number that reflects MOST CLOSELY how often you are able to do these activities:

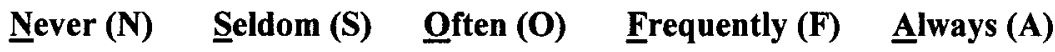

6. I help my child with homework.

7. I read to my child (or with my child).

$\begin{array}{lllll}\mathrm{N} & \mathrm{S} & \mathrm{O} & \mathrm{F} & \mathrm{A}\end{array}$

8. I attend my child's after-school activities.

$\begin{array}{lllll}\mathrm{N} & \mathrm{S} & \mathrm{O} & \mathrm{F} & \mathrm{A}\end{array}$

9. I communicate with my child's teachers by telephone.

N $\quad \mathrm{S} \quad \mathrm{O} \quad \mathrm{F} \quad \mathrm{A}$

10. I communicate with my child's teachers with notes.

11. I take my child to educational events other than school.

12. I attend the Parent Teacher Organization meetings.

13. I help with parties for children at school.

14. I help provide classroom needs for school projects.

15. I provide a study area with needed supplies at home. N

16. I visit the school to let my child know I am interested.

17. I attend parent/teacher conferences.

18. I have been involved at school since my first child started school.

N $\quad \mathrm{S} \quad \mathrm{O} \quad \mathrm{F} \quad \mathrm{A}$

N $\quad \mathrm{S} \quad \mathrm{O} \quad \mathrm{F} \quad \mathrm{A}$

$\mathrm{N} \quad \mathrm{S} \quad \mathrm{O} \quad \mathrm{F} \quad \mathrm{A}$

$\mathrm{N} \quad \mathrm{S} \quad \mathrm{O} \quad \mathrm{F} \quad \mathrm{A}$

N $\quad \mathrm{S} \quad \mathrm{O} \quad \mathrm{F} \quad \mathrm{A}$

N $\quad \mathrm{S} \quad \mathrm{O} \quad \mathrm{F} \quad \mathrm{A}$

$\begin{array}{lllll}\mathrm{N} & \mathrm{S} & \mathrm{O} & \mathrm{F} & \mathrm{A}\end{array}$

$\begin{array}{lllll}\mathrm{N} & \mathrm{S} & \mathrm{O} & \mathrm{F} & \mathrm{A}\end{array}$

$\begin{array}{lllll}\text { N } & \text { S } & \text { O } & \text { F } & \text { A }\end{array}$

$\begin{array}{lllll}\mathrm{N} & \mathrm{S} & \mathrm{O} & \mathrm{F} & \mathrm{A}\end{array}$


I C. Please answer the following question in the blanks provided.

19. In what ways are you involved in your child's education, either at home or at school? Name all that you can think of. Include school groups you are involved in and indicate any leadership positions you hold in those groups.

\section{Parent Rural Values}

II. Living in a rural area we know you are aware of what a rural community has to offer, but it would help us to know about rural values reflected in our schools.

Please rate the following statements on a scale of 1 to 5 with 1 being "strongly disagree" and 5 being "strongly agree."

1. Rural students need to be taught foreign languages.

2. Rural schools need strong arts, literature, and music programs.

3. Rural schools need strong sports programs.

4. "Book learning" is more important than "life learning."

5. A principal must be able to take control if a problem arises.

6. I'll support the principal's decision even if I don't agree with it.

7. Behavior at school would improve if we brought back paddling.

8. Reading, writing, and math should be the main subjects in school.

9. Changing books every few years is a waste of tax dollars.

10. The school should reflect our rural community standards and morals.

11. Students should be taught to be proud of their rural backgrounds.

12. "Country music" should be included in the school music program.

13. Teachers should emphasize what Kentucky has to offer.

14. Teachers should teach care of the land for future generations.

15. Vocational agriculture classes are important to our students.

16. "City" teachers who come here don't understand country people.

17. Protection of the land is an important value to teach.

18. Family farms should be protected.

Strongly

Agree

Strongly

Disagree

$\begin{array}{lllll}1 & 2 & 3 & 4 & 5 \\ 1 & 2 & 3 & 4 & 5 \\ 1 & 2 & 3 & 4 & 5\end{array}$

(n)

$\begin{array}{lllll}1 & 2 & 3 & 4 & 5 \\ 1 & 2 & 3 & 4 & 5\end{array}$


19. The country is a better place than the city to raise kids.

20. Because the family unit is in danger in America, females should be encouraged to stay at home when they marry and have children of their own.

21. A rural family's finances depend mostly on the male's income.

22. Male students should be encouraged more than female students to further their education.

23. Government attempts to control guns are against our basic rights.

24. Gun control could endanger a man's right to protect himself and his family.

25. A pick-up truck, a good hunting gun, and a nice hunting dog are status symbols in our community.

26. Most people around here who get into trouble get off with very little punishment because this is a small, country community, and everybody knows everybody

27. It's OK for a kid to miss school every once in a while to haul hay or strip tobacco.

28. It's OK for a kid to miss school every once in a while go hunting with his dad.

29. The school calendar should be shorter so kids can work on the farm.

30. A farmer's right to farm his own land can be taken away by environmentalists who promote "wetland" protection.

\section{Parent Religiosity}

III A. Often our schools teach morals and values. It would help us to know how you feel about the following issues. Please rate the following statements on a scale of 1 to 5 with 1 being "strongly disagree" and 5 being "strongly agree."

1. School takes too much time away from a child's family time.

2. School threatens the values I want my child to have.

3. Children learn most of their negative behaviors at school. Strongly
Agree Strongly Disagree

4. Issues like pre-marital sex, abortion, and homosexuality should be talked about at home, not at school.

5. Public education leads to a loss of religious faith.

6. God's creation should be taught as the beginning of the universe.

7. Bible reading at school should be an option to fiction reading.

8. We should return prayer to the classrooms.

9. We should put the Ten Commandments back in the classrooms. 
10. The Bible is without error or contradiction.

11. The only way to get to achieve eternal life is through Jesus.

12. There is a real place called heaven.

13. Hell is a place filled with unquenchable fire.

14. I know and understand God's direction for my life.

15. I can sense God's presence in my life.

16. I have heard God's "voice" or "calling."

17. I have seen or experienced personal miracles.

18. I have been "filled" with the "power" of the Holy Spirit.

19. I have received the gift of tongues, slaying in the spirit, or healing.

20. The teaching of evolution is the work of the Devil.

21. Knowledge of the Bible is a true indicator of a person's Christianity.

22. A Christian "club" should be established at school for kids.

\section{Parent Religiosity}

III B. Church is very important to some families. We would like to know how busy you are with church activities. Please answer by circling the number that MOST CLOSELY reflects how often you do the activities below.

$$
\begin{aligned}
& 1=\text { seldom (S) } \quad 2 \text { = monthly (M) } \quad 3=2 \text { or } 3 \text { times a month }(2-3 \mathrm{M}) \quad 4 \text { = weekly (W) } \\
& 5=2 \text { or } 3 \text { times a week (2-3 W) } \quad 6=\text { daily (D) } \quad 7 \text { = more than once daily (D+) }
\end{aligned}
$$

1. Attend Church

2. Attend Bible Study

3. Participate in Church related activities

4. Read the Bible

5. Study scriptures or religious material

6. Say a blessing before a meal

7. Pray aloud

8. Pray silently

9. Give money to the Church

10. Watch religious TV

11. Lead religious activities

$\begin{array}{ccrrrrl}\mathbf{S} & \mathbf{M} & \mathbf{3} \mathbf{M} & \mathbf{W} & \mathbf{2 - 3} \mathbf{W} & \mathbf{D} & \mathbf{D}+ \\ \mathbf{1} & \mathbf{2} & \mathbf{3} & \mathbf{4} & \mathbf{5} & \mathbf{6} & \mathbf{7} \\ \mathbf{1} & \mathbf{2} & \mathbf{3} & \mathbf{4} & \mathbf{5} & \mathbf{6} & \mathbf{7} \\ \mathbf{1} & \mathbf{2} & \mathbf{3} & \mathbf{4} & \mathbf{5} & \mathbf{6} & \mathbf{7} \\ \mathbf{1} & \mathbf{2} & \mathbf{3} & \mathbf{4} & \mathbf{5} & \mathbf{6} & \mathbf{7} \\ \mathbf{1} & \mathbf{2} & \mathbf{3} & \mathbf{4} & \mathbf{5} & \mathbf{6} & \mathbf{7} \\ \mathbf{1} & \mathbf{2} & \mathbf{3} & \mathbf{4} & \mathbf{5} & \mathbf{6} & \mathbf{7} \\ \mathbf{1} & \mathbf{2} & \mathbf{3} & \mathbf{4} & \mathbf{5} & \mathbf{6} & \mathbf{7} \\ \mathbf{1} & \mathbf{2} & \mathbf{3} & \mathbf{4} & \mathbf{5} & \mathbf{6} & \mathbf{7} \\ \mathbf{1} & \mathbf{2} & \mathbf{3} & \mathbf{4} & \mathbf{5} & \mathbf{6} & \mathbf{7} \\ \mathbf{1} & \mathbf{2} & \mathbf{3} & \mathbf{4} & \mathbf{5} & \mathbf{6} & \mathbf{7} \\ \mathbf{1} & \mathbf{2} & \mathbf{3} & \mathbf{4} & \mathbf{5} & \mathbf{6} & \mathbf{7}\end{array}$




\section{Parent Primary Affiliation}

IV A. Our friends can come from anywhere. We may choose them from work, church, school, civic organizations, or any other community group. Please tell us for which situations you would most likely choose friends. This information will help us to know if school has been successful in creating a friendly environment.

Below you will see a LIST of situations for which you might need a friend. In the COLUMNS you will see groups that might offer friends to you.

CIRCLE a number for each situation from all three groups that BEST reveals your feelings about choosing a friend from each group for the 17 situations listed.

Choose a number from each group.

1. Situations Church

School

Other (civic, social)

2. Being best friend

$\begin{array}{lllllllllllllll}1 & 2 & 3 & 4 & 5 & 1 & 2 & 3 & 4 & 5 & 1 & 2 & 3 & 4 & 5\end{array}$

3. Helping move

$\begin{array}{lllllllllllllll}1 & 2 & 3 & 4 & 5 & 1 & 2 & 3 & 4 & 5 & 1 & 2 & 3 & 4 & 5\end{array}$

4. Chaperoning a party

$\begin{array}{lllllllllllllll}1 & 2 & 3 & 4 & 5 & 1 & 2 & 3 & 4 & 5 & 1 & 2 & 3 & 4 & 5\end{array}$

5. Shopping for clothes

$\begin{array}{lllllllllllllll}1 & 2 & 3 & 4 & 5 & 1 & 2 & 3 & 4 & 5 & 1 & 2 & 3 & 4 & 5\end{array}$

6. Attending a funeral

$\begin{array}{lllllllllllllll}1 & 2 & 3 & 4 & 5 & 1 & 2 & 3 & 4 & 5 & 1 & 2 & 3 & 4 & 5\end{array}$

7. Vacationing

$\begin{array}{lllllllllllllll}1 & 2 & 3 & 4 & 5 & 1 & 2 & 3 & 4 & 5 & 1 & 2 & 3 & 4 & 5\end{array}$

8. Playing ball

9. Meeting for lunch

$\begin{array}{lllllllllllllll}1 & 2 & 3 & 4 & 5 & 1 & 2 & 3 & 4 & 5 & 1 & 2 & 3 & 4 & 5\end{array}$

10. Talking on the phone

$\begin{array}{lllllllllllllll}1 & 2 & 3 & 4 & 5 & 1 & 2 & 3 & 4 & 5 & 1 & 2 & 3 & 4 & 5\end{array}$

11. Visiting hospital

$\begin{array}{lllllllllllllll}1 & 2 & 3 & 4 & 5 & 1 & 2 & 3 & 4 & 5 & 1 & 2 & 3 & 4 & 5\end{array}$

12. Surviving crisis

$\begin{array}{lllllllllllllll}1 & 2 & 3 & 4 & 5 & 1 & 2 & 3 & 4 & 5 & 1 & 2 & 3 & 4 & 5\end{array}$

13. Exercising

$\begin{array}{lllllllllllllll}1 & 2 & 3 & 4 & 5 & 1 & 2 & 3 & 4 & 5 & 1 & 2 & 3 & 4 & 5\end{array}$

14. Naming child after

$\begin{array}{lllllllllllllll}1 & 2 & 3 & 4 & 5 & 1 & 2 & 3 & 4 & 5 & 1 & 2 & 3 & 4 & 5\end{array}$

15. Borrowing a car

$\begin{array}{lllllllllllllll}1 & 2 & 3 & 4 & 5 & 1 & 2 & 3 & 4 & 5 & 1 & 2 & 3 & 4 & 5\end{array}$

16. Grieving death

$\begin{array}{lllllllllllllll}1 & 2 & 3 & 4 & 5 & 1 & 2 & 3 & 4 & 5 & 1 & 2 & 3 & 4 & 5\end{array}$

17. Celebration an event

$\begin{array}{lllllllllllllll}1 & 2 & 3 & 4 & 5 & 1 & 2 & 3 & 4 & 5 & 1 & 2 & 3 & 4 & 5\end{array}$

18. Making a decision

$\begin{array}{lllllllllllllll}1 & 2 & 3 & 4 & 5 & 1 & 2 & 3 & 4 & 5 & 1 & 2 & 3 & 4 & 5\end{array}$

$1=$ NO CHANCE OF CHOOSING FROM THIS GROUP

2 = SOME CHANCE OF CHOOSING FROM THIS GROUP

$3=$ NEUTRAL

$4=$ GOOD CHANCE OF CHOOSING FROM THIS GROUP

$5=$ EXCELLENT CHANCE OF CHOOSING FROM THIS GROUP

(continued) 


\section{Parent Affiliation Bond}

IV B. How you feel about your participation in activities is important in planning for school activities. We would like to know how you feel about your involvement in church, school, social (informal get-togethers), and civic (Lions Club, Women's Club, etc.) activities. Please, CIRCLE the number on the scale that BEST reflects how you feel. There is no right or wrong, correct or incorrect answer. Your answer will tell us only whether you feel more like an "observer" or more like a "player."

The number 7 is as far as you can go towards either end of the line, and the number 3 is neutral.

1. At church I am the

2. In school activities I am the

3. In social groups I am the

4. In civic groups I am the

5. At church I am the

6. At school I am the

7. In social groups I am the

8. In civic groups I am the

9. Church people are

10. School people are

11. Social group people are

12. Civic group people are

13. At church I am

14. At school I am

15. In social groups I am

16. In civic groups I am

17. At church I know more

18. At school I know more

19. In social groups I know more

20. In civic groups I know more

$\begin{array}{rllllllllll}\text { audience } & 7 & 6 & 5 & 4 & 3 & 4 & 5 & 6 & 7 & \text { participant } \\ \text { audience } & 7 & 6 & 5 & 4 & 3 & 4 & 5 & 6 & 7 & \text { participant } \\ & & & & & & & & & & \\ \text { audience } & 7 & 6 & 5 & 4 & 3 & 4 & 5 & 6 & 7 & \text { participant } \\ \text { audience } & 7 & 6 & 5 & 4 & 3 & 4 & 5 & 6 & 7 & \text { participant } \\ \text { outsider } & 7 & 6 & 5 & 4 & 3 & 4 & 5 & 6 & 7 & \text { insider } \\ \text { outsider } & 7 & 6 & 5 & 4 & 3 & 4 & 5 & 6 & 7 & \text { insider } \\ \text { outsider } & 7 & 6 & 5 & 4 & 3 & 4 & 5 & 6 & 7 & \text { insider } \\ \text { outsider } & 7 & 6 & 5 & 4 & 3 & 4 & 5 & 6 & 7 & \text { insider } \\ \text { like me } & 7 & 6 & 5 & 4 & 3 & 4 & 5 & 6 & 7 & \text { like me } \\ \text { like me } & 7 & 6 & 5 & 4 & 3 & 4 & 5 & 6 & 7 & \text { like me } \\ \text { like me } & 7 & 6 & 5 & 4 & 3 & 4 & 5 & 6 & 7 & \text { like me } \\ \text { like me } & 7 & 6 & 5 & 4 & 3 & 4 & 5 & 6 & 7 & \text { like me } \\ \text { unneeded } & 7 & 6 & 5 & 4 & 3 & 4 & 5 & 6 & 7 & \text { needed } \\ \text { unneeded } & 7 & 6 & 5 & 4 & 3 & 4 & 5 & 6 & 7 & \text { needed } \\ \text { unneeded } & 7 & 6 & 5 & 4 & 3 & 4 & 5 & 6 & 7 & \text { needed } \\ \text { unneeded } & 7 & 6 & 5 & 4 & 3 & 4 & 5 & 6 & 7 & \text { needed } \\ \text { close friends } & 7 & 6 & 5 & 4 & 3 & 4 & 5 & 6 & 7 & \text { close friend } \\ \text { close friends } & 7 & 6 & 5 & 4 & 3 & 4 & 5 & 6 & 7 & \text { close friend } \\ \text { close friends } & 7 & 6 & 5 & 4 & 3 & 4 & 5 & 6 & 7 & \text { close friend } \\ & & & & & & & & & \\ \text { close friends } & 7 & 6 & 5 & 4 & 3 & 4 & 5 & 6 & 7 & \text { close friend }\end{array}$

\section{Parent Demographics}

A. It is important for us to include as many people and as many different kinds of families as possible in our survey. Please help us to know what kinds of families we are surveying by giving BEST answer for each question below:

1. Are you male or female?

2. What is your age?

3. How many children are in the family?

4. How many children are in school (P-12)?

5. How many pre-school children live in your home?

6. How many adult parents or other "caretakers" live in your home?

7. How many adults who are disabled or unable to care for themselves live in your home?

8. Is the head of the house male or female, or are the duties shared? (Please CHECK the best answer.) M Shared 
9. What is the highest education level you have completed? (Please CHECK the best answer.)

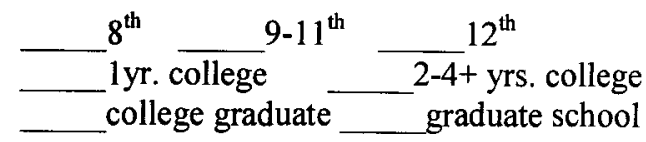

V B. Many of the families we serve cannot be as involved in schools as they would like because of family structures. Please check all that apply to your family.

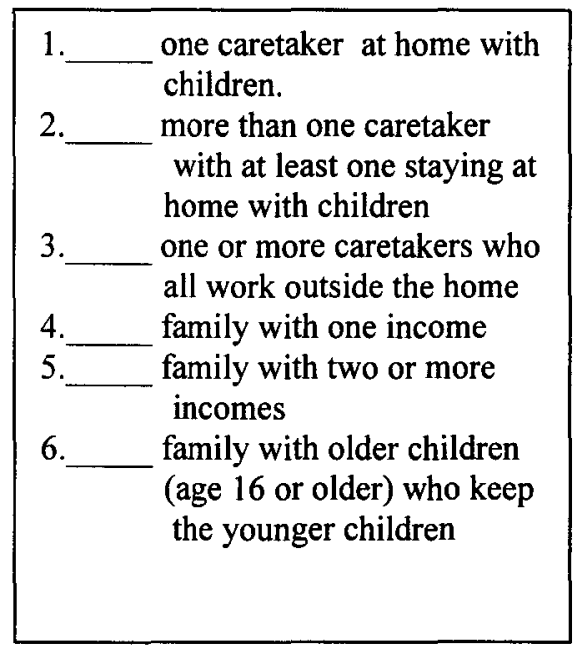

$\begin{array}{|ll|}7 . \quad \text { family with transportation only } & \text { for the working caretaker } \\ 8 . \quad \begin{array}{l}\text { family with senior citizen as head } \\ \text { of house } \\ \text { family representing two } \\ \text { generations (parent/child) } \\ \text { family representing more than } \\ \text { two generations } \\ \text { (parent/child/grandchild) } \\ \text { family with at least one absent } \\ \text { parent }\end{array} \\ \end{array}$

V C. What is an estimate of your family income (CHECK one)?

$$
\begin{aligned}
& 1 . \quad \$ 0-15,000 \\
& 2 . \quad \$ 15,001--30,000 \\
& \text { 3.__ } \$ 30,001-45,000
\end{aligned}
$$

$$
\begin{aligned}
& \text { 4.___ } \$ 45,001--60,000 \\
& \text { 5.___ } \$ 60,001--75,000 \\
& 6 . ـ \$ 75,001--90,000+
\end{aligned}
$$

You have finished the survey! Thank you so much for helping! If you have any questions and/or comments, you may contact E Carolyn Tucker at ectucker@kih.net or 639-5107. 


\section{APPENDIX P}

\section{Student View of Parent Involvement}

I A. We know that parents want the best education possible for children. Because Parent Involvement is important for student achievement, it would help us to know how you feel about some of the opportunities for parent involvement at your school. Please rate the following statements on a scale of 1 to 5 with 1 being "strongly disagree" and 5 being "strongly agree." CIRCLE the number you choose.

1. The school values my parent's participation in my school activities.

2. The staff at school welcomes my parents when they visit.

$\begin{array}{lllll}1 & 2 & 3 & 4 & 5 \\ 1 & 2 & 3 & 4 & 5\end{array}$

3. My teacher(s) considers my parents important in my education. $\quad \begin{array}{llllll}1 & 2 & 3 & 4 & 5\end{array}$

4. It is important to find the time to be involved in my child's school. $\begin{array}{lllllll}1 & 2 & 3 & 4 & 5\end{array}$

$\begin{array}{llllllll}\text { 5. I learn more when my parents are involved with my schooling. } & 1 & 2 & 3 & 4 & 5\end{array}$

I B. Please CIRCLE the number that reflects MOST CLOSELY how often your parents are able to do these activities:

$\underline{\text { Never (N) }} \quad \underline{\text { Seldom (S) }}$ Often (O) Frequently (F) Always (A)

6. I help my child with homework.

$\begin{array}{lllll}\mathrm{N} & \mathrm{S} & \mathrm{O} & \mathrm{F} & \mathrm{A}\end{array}$

7. I read to my child (or with my child).

$\begin{array}{lllll}\mathrm{N} & \mathrm{S} & \mathrm{O} & \mathrm{F} & \mathrm{A}\end{array}$

8. I attend my child's after-school activities.

9. I communicate with my child's teachers by telephone.

10. I communicate with my child's teachers with notes.

11. I take my child to educational events other than school.

12. I attend the Parent Teacher Organization meetings.

$\mathrm{N}$

13. I help with parties for children at school.

N $\quad \mathrm{S} \quad \mathrm{O} \quad \mathrm{F} \quad \mathrm{A}$

14. I help provide classroom needs for school projects.

$\mathrm{N}$

$\mathrm{S}$

O F A

15. I provide a study area with needed supplies at home. N

16. I visit the school to let my child know I am interested.

17. I attend parent/teacher conferences.

18. I have been involved at school since my first child started school.

N $\quad \mathrm{S} \quad \mathrm{O} \quad \mathrm{F} \quad \mathrm{A}$

$\begin{array}{lllll}\mathrm{N} & \mathrm{S} & \mathrm{O} & \mathrm{F} & \mathrm{A}\end{array}$

N $\quad \mathrm{S} \quad \mathrm{O} \quad \mathrm{F} \quad \mathrm{A}$

$\begin{array}{lllll}\mathrm{N} & \mathrm{S} & \mathrm{O} & \mathrm{F} & \mathrm{A}\end{array}$

(continued) 
I C. Please answer the following question in the blanks provided.

19. In what ways are your parents involved in your education, either at home or at school? Name all that you can think of. Include school groups they are involved in and indicate any leadership positions they hold in those groups.

\section{Student Rural Values}

II. Living in a rural area we know you are aware of what a rural community has to offer, but it would help us to know about rural values reflected in our schools. Please rate the following statements on a scale of 1 to 5 with 1 being "strongly disagree" and 5 being "strongly agree."

1. Rural students need to be taught foreign languages.

$\begin{array}{ll}\text { Strongly } & \text { Strongly } \\ \text { Disagree } & \text { Agree }\end{array}$

2. Rural schools need strong arts, literature, and music programs.

3. Rural schools need strong sports programs.

4. "Book learning" is more important than "life learning."

5. A principal must be able to take control if a problem arises.

6. I'll support the principal's decision even if I don't agree with it.

$\begin{array}{lllll}1 & 2 & 3 & 4 & 5 \\ 1 & 2 & 3 & 4 & 5\end{array}$

7. Behavior at school would improve if we brought back paddling.

8. Reading, writing, and math should be the main subjects in school.

9. Changing books every few years is a waste of tax dollars.

10. The school should reflect our rural community standards and morals.

11. Students should be taught to be proud of their rural backgrounds.

12. "Country music" should be included in the school music program.

13. Teachers should emphasize what Kentucky has to offer.

14. Teachers should teach care of the land for future generations.

15. Vocational agriculture classes are important to our students.

16. "City" teachers who come here don't understand country people.

17. Protection of the land is an important value to teach.

18. Family farms should be protected.

19. The country is a better place than the city to raise kids.

\begin{tabular}{|c|c|c|c|}
\hline 1 & 2 & 3 & 4 \\
\hline 1 & 2 & 3 & 4 \\
\hline 1 & 2 & 3 & 4 \\
\hline 1 & 2 & 3 & 4 \\
\hline 1 & 2 & 3 & 4 \\
\hline 1 & 2 & 3 & 4 \\
\hline 1 & 2 & 3 & 4 \\
\hline 1 & 2 & 3 & 4 \\
\hline 1 & 2 & 3 & 4 \\
\hline 1 & 2 & 3 & 4 \\
\hline 1 & 2 & 3 & 4 \\
\hline 1 & 2 & 3 & 4 \\
\hline 1 & 2 & 3 & 4 \\
\hline 1 & 2 & 3 & 4 \\
\hline 1 & 2 & 3 & 4 \\
\hline & 2 & 3 & 4 \\
\hline 1 & 2 & 3 & 4 \\
\hline
\end{tabular}


20. Because the family unit is in danger in America, females should be encouraged to stay at home when they marry and have children of their own.

21. A rural family's finances depend mostly on the male's income.

22. Male students should be encouraged more than female students to further their education.

23. Government attempts to control guns are against our basic rights.

24. Gun control could endanger a man's right to protect himself and his family.

25. A pick-up truck, a good hunting gun, and a nice hunting dog are status symbols in our community.

26. Most people around here who get into trouble get off with very little punishment because this is a small, country community, and everybody knows everybody.

27. It's OK for a kid to miss school every once in a while to haul hay or strip tobacco.

28. It's OK for a kid to miss school every once in a while to go hunting with his dad.

29. The school calendar should be shorter so kids can work on the farm.

30. A farmer's right to farm his own land can be taken away by environmentalists who promote "wetland" protection.

$\begin{array}{lllll}1 & 2 & 3 & 4 & 5 \\ 1 & 2 & 3 & 4 & 5 \\ 1 & 2 & 3 & 4 & 5 \\ 1 & 2 & 3 & 4 & 5 \\ 1 & 2 & 3 & 4 & 5 \\ 1 & 2 & 3 & 4 & 5 \\ 1 & 2 & 3 & 4 & 5 \\ 1 & 2 & 3 & 4 & 5 \\ 1 & 2 & 3 & 4 & 5 \\ 1 & 2 & 3 & 4 & 5 \\ 1 & 2 & 3 & 4 & 5\end{array}$

\section{Student Religiosity}

III A. Often our schools teach morals and values. It would help us to know how you feel about the following issues. Please rate the following statements on a scale of 1 to 5 with 1 being "strongly disagree" and 5 being "strongly agree."

1. School takes too much time away from a child's family time.

2. School threatens the values I want my child to have.

3. Children learn most of their negative behaviors at school.

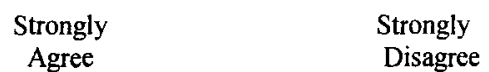

4. Issues like pre-marital sex, abortion, and homosexuality should be talked about at home, not at school.

5. Public education leads to a loss of religious faith.

6. God's creation should be taught as the beginning of the universe.

7. Bible reading at school should be an option to fiction reading.

8. We should return prayer to the classrooms.

9. We should put the Ten Commandments back in the Agree Disagree classrooms. 
10. The Bible is without error or contradiction.

11. The only way to get to achieve eternal life is through Jesus.

12. There is a real place called heaven.

13. Hell is a place filled with unquenchable fire.

14. I know and understand God's direction for my life.

15. I can sense God's presence in my life.

16. I have heard God's "voice" or "calling."

17. I have seen or experienced personal miracles.

18. I have been "filled" with the "power" of the Holy Spirit.

19. I have received the gift of tongues, slaying in the spirit, or healing.

20. The teaching of evolution is the work of the Devil.

21. Knowledge of the Bible is a true indicator of a person's Christianity.

22. A Christian "club" should be established at school for kids. $\begin{array}{lllll}1 & 2 & 3 & 4 & 5\end{array}$

$\begin{array}{lllll}1 & 2 & 3 & 4 & 5\end{array}$

$\begin{array}{lllll}1 & 2 & 3 & 4 & 5\end{array}$

$\begin{array}{lllll}1 & 2 & 3 & 4 & 5\end{array}$

$\begin{array}{lllll}1 & 2 & 3 & 4 & 5\end{array}$

$\begin{array}{lllll}1 & 2 & 3 & 4 & 5\end{array}$

$\begin{array}{lllll}1 & 2 & 3 & 4 & 5\end{array}$

$\begin{array}{lllll}1 & 2 & 3 & 4 & 5\end{array}$

$\begin{array}{lllll}1 & 2 & 3 & 4 & 5\end{array}$

$\begin{array}{lllll}1 & 2 & 3 & 4 & 5\end{array}$

$\begin{array}{lllll}1 & 2 & 3 & 4 & 5\end{array}$

$\begin{array}{lllll}1 & 2 & 3 & 4 & 5\end{array}$

$\begin{array}{lllll}1 & 2 & 3 & 4 & 5\end{array}$ 


\section{Student Primary Affiliation}

IV A. Our friends can come from anywhere. We may choose them from work, church, school, civic organizations, or any other community group. Please tell us for which situations you would most likely choose friends. This information will help us to know if school has been successful in creating a friendly environment. Below you will see a LIST of situations for which you might need a friend. In the COLUMNS you will see groups that might offer friends to you. CIRCLE a number for each situation from all three groups that BEST reveals your feelings about choosing a friend from each group for the 17 situations listed.

Choose a number from each group.

1. Situations Church School Other (civic, social)

2. Being best friend $\begin{array}{lllllllllllllll}1 & 2 & 3 & 4 & 5 & 1 & 2 & 3 & 4 & 5 & 1 & 2 & 3 & 4 & 5\end{array}$

3. Helping move $\begin{array}{lllllllllllllll}1 & 2 & 3 & 4 & 5 & 1 & 2 & 3 & 4 & 5 & 1 & 2 & 3 & 4 & 5\end{array}$

4. Chaperoning a party $\begin{array}{lllllllllllllll}1 & 2 & 3 & 4 & 5 & 1 & 2 & 3 & 4 & 5 & 1 & 2 & 3 & 4 & 5\end{array}$

5. Shopping for clothes

$\begin{array}{lllllllllllllll}1 & 2 & 3 & 4 & 5 & 1 & 2 & 3 & 4 & 5 & 1 & 2 & 3 & 4 & 5\end{array}$

6. Attending a funeral

$\begin{array}{lllllllllllllll}1 & 2 & 3 & 4 & 5 & 1 & 2 & 3 & 4 & 5 & 1 & 2 & 3 & 4 & 5\end{array}$

7. Vacationing

$\begin{array}{lllllllllllllll}1 & 2 & 3 & 4 & 5 & 1 & 2 & 3 & 4 & 5 & 1 & 2 & 3 & 4 & 5\end{array}$

8. Playing ball

9. Meeting for lunch

$\begin{array}{lllllllllllllll}1 & 2 & 3 & 4 & 5 & 1 & 2 & 3 & 4 & 5 & 1 & 2 & 3 & 4 & 5\end{array}$

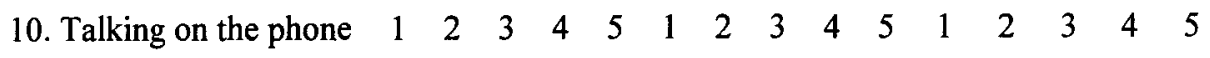

11. Visiting hospital $\quad \begin{array}{llllllllllllllll} & 1 & 2 & 3 & 4 & 5 & 1 & 2 & 3 & 4 & 5 & 1 & 2 & 3 & 4 & 5\end{array}$

$\begin{array}{llllllllllllllll}\text { 12. Surviving crisis } & 1 & 2 & 3 & 4 & 5 & 1 & 2 & 3 & 4 & 5 & 1 & 2 & 3 & 4 & 5\end{array}$

$\begin{array}{llllllllllllllll}\text { 13. Exercising } & 1 & 2 & 3 & 4 & 5 & 1 & 2 & 3 & 4 & 5 & 1 & 2 & 3 & 4 & 5\end{array}$

$\begin{array}{llllllllllllllll}\text { 14. Naming child after } & 1 & 2 & 3 & 4 & 5 & 1 & 2 & 3 & 4 & 5 & 1 & 2 & 3 & 4 & 5\end{array}$

$\begin{array}{llllllllllllllll}\text { 15. Borrowing a car } & 1 & 2 & 3 & 4 & 5 & 1 & 2 & 3 & 4 & 5 & 1 & 2 & 3 & 4 & 5\end{array}$

$\begin{array}{llllllllllllllll}\text { 16. Grieving death } & 1 & 2 & 3 & 4 & 5 & 1 & 2 & 3 & 4 & 5 & 1 & 2 & 3 & 4 & 5\end{array}$

$\begin{array}{llllllllllllllll}\text { 17. Celebration an event } & 1 & 2 & 3 & 4 & 5 & 1 & 2 & 3 & 4 & 5 & 1 & 2 & 3 & 4 & 5\end{array}$

$\begin{array}{llllllllllllllll}\text { 18. Making a decision } & 1 & 2 & 3 & 4 & 5 & 1 & 2 & 3 & 4 & 5 & 1 & 2 & 3 & 4 & 5\end{array}$

$$
\begin{aligned}
& 1=\text { NO CHANCE OF CHOOSING FROM THIS GROUP } \\
& 2=\text { SOME CHANCE OF CHOOSING FROM THIS GROUP } \\
& 3=\text { NEUTRAL } \\
& 4=\text { GOOD CHANCE OF CHOOSING FROM THIS GROUP } \\
& 5=\text { EXCELLENT CHANCE OF CHOOSING FROM THIS GROUP (continued) }
\end{aligned}
$$




\section{Student Affiliation Bond}

IV B. How you feel about your participation in activities is important in planning for school activities. We would like to know how you feel about your involvement in church, school, social (informal get-togethers), and civic (Lions Club, Women's Club, etc.) activities. Please, CIRCLE the number on the scale that BEST reflects how you feel. There is no right or wrong, correct or incorrect answer. Your answer will tell us only whether you feel more like an "observer" or more like a "player."

The number 7 is as far as you can go towards either end of the line, and the number 3 is neutral.

1. At church I am the

2. In school activities I am the

3. In social groups I am the

4. In civic groups I am the

5. At church I am the

6. At school I am the

7. In social groups I am the

8. In civic groups I am the

9. Church people are

10. School people are

11. Social group people are

12. Civic group people are

13. At church I am

14. At school I am

15. In social groups I am

16. In civic groups I am

17. At church I know more

18. At school I know more

19. In social groups I know more

20. In civic groups I know more

$\begin{array}{rllllllllll}\text { audience } & 7 & 6 & 5 & 4 & 3 & 4 & 5 & 6 & 7 & \text { participant } \\ \text { audience } & 7 & 6 & 5 & 4 & 3 & 4 & 5 & 6 & 7 & \text { participant } \\ & & & & & & & & & & \\ \text { audience } & 7 & 6 & 5 & 4 & 3 & 4 & 5 & 6 & 7 & \text { participant } \\ \text { audience } & 7 & 6 & 5 & 4 & 3 & 4 & 5 & 6 & 7 & \text { participant } \\ \text { outsider } & 7 & 6 & 5 & 4 & 3 & 4 & 5 & 6 & 7 & \text { insider } \\ \text { outsider } & 7 & 6 & 5 & 4 & 3 & 4 & 5 & 6 & 7 & \text { insider } \\ \text { outsider } & 7 & 6 & 5 & 4 & 3 & 4 & 5 & 6 & 7 & \text { insider } \\ \text { outsider } & 7 & 6 & 5 & 4 & 3 & 4 & 5 & 6 & 7 & \text { insider } \\ \text { like me } & 7 & 6 & 5 & 4 & 3 & 4 & 5 & 6 & 7 & \text { like me } \\ \text { like me } & 7 & 6 & 5 & 4 & 3 & 4 & 5 & 6 & 7 & \text { like me } \\ \text { like me } & 7 & 6 & 5 & 4 & 3 & 4 & 5 & 6 & 7 & \text { like me } \\ \text { like me } & 7 & 6 & 5 & 4 & 3 & 4 & 5 & 6 & 7 & \text { like me } \\ \text { unneeded } & 7 & 6 & 5 & 4 & 3 & 4 & 5 & 6 & 7 & \text { needed } \\ \text { unneeded } & 7 & 6 & 5 & 4 & 3 & 4 & 5 & 6 & 7 & \text { needed } \\ \text { unneeded } & 7 & 6 & 5 & 4 & 3 & 4 & 5 & 6 & 7 & \text { needed } \\ \text { unneeded } & 7 & 6 & 5 & 4 & 3 & 4 & 5 & 6 & 7 & \text { needed } \\ \text { close friends } & 7 & 6 & 5 & 4 & 3 & 4 & 5 & 6 & 7 & \text { close friend } \\ \text { close friends } & 7 & 6 & 5 & 4 & 3 & 4 & 5 & 6 & 7 & \text { close friend } \\ \text { close friends } & 7 & 6 & 5 & 4 & 3 & 4 & 5 & 6 & 7 & \text { close friend } \\ & & & & & & & & & & \\ \text { close friends } & 7 & 6 & 5 & 4 & 3 & 4 & 5 & 6 & 7 & \text { close friend }\end{array}$

\section{Student Activities}

V. We would like to know what activities you are involved in. Please CHECK any activity listed below that you participate in.

1. What activities (other than class work) are you involved in at school? (Check ALL that apply.)

baseball
basketball
fostball
track
cross country
softball
tennis
golf

soccer
academics
speech
math team
apprenticeship
ESS
cheerleading
flag team

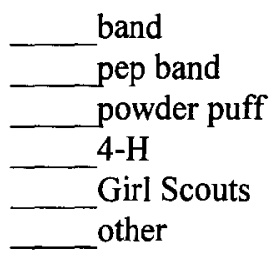

(continued) 
2. What other organized activities are you involved in after school? (Check ALL that apply.)

motor cross

archery club

Rainbows

hunt/fish club

work at home

w__ work for money
horse riding
missions
church youth
pony pull

martial arts club dance team gymnastics other

3. What leadership roles (president, treasurer, team leader, etc.) do you hold in any of the activities you do? (Name any leadership role and the organization, at school or after school.)

4. Please list any activities you are involved in that are not covered by the answers above.

You have finished the survey! Thank you so much for helping! If you have any questions and/or comments, you may contact $E$ Carolyn Tucker at ectucker@kih.net or 639-5107.

The following is for researcher use only.

GPA

KIRIS

SDI

CATS 


\section{APPENDIX Q}

\section{Parent Survey Scoring Protocol}

Demographics (FS)

Section V A.
\# $\quad$ Statement
Response Points
3 How many children are in the family?
1 per
6 How many adult parents or other "caretakers"
1 per
live in your home?
7 How many adults who are disabled or unable to care for themselves live in your home?

Family Structure Score derived from scores of \#3, \#6, and \#7 above: $\frac{3+7}{6}=$ FS Note. This ratio is negatively associated with family resources, such that a larger number of dependents yields a higher ration. It is not possible to invert the measures because a couple or single with no dependents would result in an undefined function ( 0 in denominator). 
Demographics (SES)

Section V A.

\# Statement

9 What is the highest education level you have completed?
Response

Points

\begin{tabular}{ll}
$8^{\text {th }}$ & 1 \\
$-9-11$ & 2 \\
$-12^{\text {th }}$ & 3 \\
$-\quad$ some college & 4 \\
\hline college graduate & 5 \\
\hline graduate school + & 6
\end{tabular}

Section V C.
Response

Points

What is an estimate of your family income?

$\begin{array}{ll}1 & \$ 0-15,000 \\ 2 & \$ 15,001-30,000 \\ 3 & \$ 30,001-45,000 \\ 4 & \$ 45,001-60,000 \\ 5 & \$ 60,001-75,000 \\ 6 & \$ 75,001-90,000+\end{array}$

Check

Check

Check

Check

Check

Check

Socioeconomic Status (SES) is computed for each family; for each parent, the point values for education level are added to the point value for family income. The resulting scale ranges from 2-12.

$\mathrm{H} / \mathrm{M} / \mathrm{L}$ Categories

$$
\begin{gathered}
H=9-11 \\
M=6-8 \\
L=3-5
\end{gathered}
$$




\section{APPENDIX R}

\section{Parent/Student Survey Scoring Protocol}

Note. For all scales in this appendix, the parent version and student version of the construct are identical. The only difference is who filled out the survey: parent or student.

\section{Parent Rural Values: Anti-Intellectualism (RV-AI) Section II}

\#

1 Rural students need to be taught foreign languages.

2 Rural students need strong arts, literature, and music programs.

3 Rural schools need strong sports programs.

4 "Book learning" is more important than "life learning."

8 Reading, writing, and math should be the main subjects in school.

9 Changing books every few years is a waste of tax dollars.

29 The school calendar should be shorter so kids can work on the farm.
Response

$1-5$

Reverse Score

$1-5$

Reverse Score

$1-5$

$1-5$

Reverse Score

$1-5$

$1-5$

$1-5$

Likert Scale: $1-5 /$ Strongly Disagree to Strongly Agree

Parent Rural Values: Social Conservatism/Traditionalism (RV-SC/T)

\#

5 A principal must be able to take control if a problem arises.

6 I'll support the principal's decision even if I don't agree with it.

7 Behavior at school would improve if we brought back paddling.

20 Because the family unit is in danger in America, females should be encouraged to stay at home when they marry and have children of their own.

21 A rural family's finances depend mostly on the male's income

22 Male students should be encouraged more than female students to further their education.

Likert Scale: $1-5 /$ Strongly Disagree to Strongly Agree
Response

$1-5$

$1-5$

$1-5$

$1-5$

$1-5$

$1-5$

(continued) 
Parent Rural Values: Ethnocentricity (RV-E)

10 The school should reflect our rural community standards and morals. $1-5$

11 Students should be taught to be proud of their rural backgrounds. $1-5$

12 "Country music" should be included in the school music program. $1-5$

13 Teachers should emphasize what Kentucky has to offer. $1-5$

16 "City" teachers who come here don't understand country people. $1-5$

19 The country is a better place than the city to raise kids. $1-5$

Likert Scale: 1-5/Strongly Disagree to Strongly Agree

Parent Rural Values: Stewardship of Earth (RV-SE)

\# $\quad$ Statement

Response

14 Teachers should teach care of the land for future generations.

$1-5$

15 Vocational agriculture classes are important to our students.

$1-5$

17 Protection of the land is an important value to teach.

$1-5$

18 Family farms should be protected.

$1-5$

30 A farmer's right to farm his own land can be taken away by $1-5$ environmentalists who promote "wetland" protection.

Likert Scale: $1-5 /$ Strongly Disagree to Strongly Agree 
23 Government attempts to control guns are against our basic rights.

24 Gun control could endanger a man's right to protect himself and his family.

25 A pick-up truck, a good hunting gun, and a nice hunting dog are status symbols in our community.

26 Most people around here who get into trouble get off with very little punishment because this is a small, country community, and everybody knows everybody.

27 It's OK for a kid to miss school every once in a while to haul hay or $1-5$ strip tobacco.

28 It's OK for a kid to miss school every once in a while to go hunting $1-5$ with his dad.

Likert Scale: $1-5 /$ Strongly Disagree to Strongly Agree

\section{Parent Religiosity: Belief (PR-BEL) Sub-construct School as Threat}

\section{Section III A.}

\# Statement

1 School takes too much time away from a child's family time. $1-5$

2 School threatens the values I want my child to have. $1-5$

3 Children learn most of their negative behaviors at school. $1-5$

4 Issues like pre-marital sex, abortion, and homosexuality should be $1-5$ talked about at home, not at school.

5 Public education leads to a loss of religious faith. $1-5$

Likert Scale: 1-5/Strongly Disagree to Strongly Agree 
Parent Religiosity: Belief (PR-BEL)

Sub-construct: Integration of Religion into School

\# Statement

Response

6 God's creation should be taught as the beginning of the universe. $1-5$

7 Bible reading at school should be an option to fiction reading. $\quad 1-5$

8 We should return prayer to the classrooms. $1-5$

9 We should put the Ten Commandments back in the classrooms. $1-5$

22 A Christian "club" should be established at school for kids. $1-5$

Likert Scale: $1-5 /$ Strongly Disagree to Strongly Agree

Parent Religiosity: Belief (PR-BEL)

Sub-construct: Literalist Belief

\# $\quad$ Statement

Response

10 The Bible is without error or contradiction.

$1-5$

11 The only way to get to achieve eternal life is through Jesus. $1-5$

12 There is a real place called heaven. $1-5$

13 Hell is a place filled with unquenchable fire. $1-5$

20 The teaching of evolution is the work of the Devil. $1-5$

21 Knowledge of the Bible is a true indicator of a person's Christianity. $1-5$ Likert Scale: $1-5 /$ Strongly Disagree to Strongly Agree 
Parent Religiosity: Experience (PR-EXP)

\# Statement

Response

14 I know and understand God's direction for my life

$1-5$

15 I can sense God's presence in my life.

$1-5$

16 I have heard God's "voice" or "calling."

$1-5$

17 I have seen or experienced personal miracles.

$1-5$

18 I have been "filled" with the "power" of the Holy Spirit.

$1-5$

19 I have received the gift of tongues, slaying in the spirit, or healing.

1-5

Likert Scale: $1-5 /$ Strongly Disagree to Strongly Agree 


\section{Section III B}

\section{Parent Religiosity: Ritual (PR-RIT)}

$1=$ seldom (S) $2=$ monthly (M) $3=2$ or 3 times a month $(2-3 \mathrm{M}) \quad 4=$ weekly $(\mathrm{W})$ $5=2$ or 3 times a week $(2-3 \mathrm{~W}) \quad 6=-$ daily (D) $\quad 7=$ more than once daily (D+)

1 Attend Church

2 Attend Bible Study

3 Participate in Church related activities

4 Read the Bible

5 Study scriptures or religious material

6 Say a blessing before a meal

7 Pray aloud

8 Pray silently

9 Give money to the church

10 Watch religious TV

11 Lead religious activities

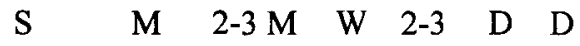

$\begin{array}{lllllll}1 & 2 & 3 & 4 & 5 & 6 & 7\end{array}$

$\begin{array}{lllllll}1 & 2 & 3 & 4 & 5 & 6 & 7\end{array}$

$\begin{array}{llllllll}1 & 2 & 3 & 4 & 5 & 6 & 7\end{array}$

$\begin{array}{lllllll}1 & 2 & 3 & 4 & 5 & 6 & 7\end{array}$

$\begin{array}{lllllll}1 & 2 & 3 & 4 & 5 & 6 & 7\end{array}$

$\begin{array}{lllllll}1 & 2 & 3 & 4 & 5 & 6 & 7\end{array}$

$\begin{array}{lllllll}1 & 2 & 3 & 4 & 5 & 6 & 7\end{array}$

$\begin{array}{lllllll}1 & 2 & 3 & 4 & 5 & 6 & 7\end{array}$

$\begin{array}{lllllll}1 & 2 & 3 & 4 & 5 & 6 & 7\end{array}$

$\begin{array}{lllllll}1 & 2 & 3 & 4 & 5 & 6 & 7\end{array}$

$\begin{array}{lllllll}1 & 2 & 3 & 4 & 5 & 6 & 7\end{array}$

Behavioral Frequency Scale
1 = Seldom $(\mathrm{S})$
$5=2$ or 3 times a week $(2-3 \mathrm{~W})$
2 = Monthly (M)
$6=$ Daily $(\mathrm{D})$
$3=2$ or 3 times a month (2-3 M)
$7=$ More than once daily $(\mathrm{D}+)$
$4=$ Weekly $(W)$ 


\section{Parent Religiosity: Affiliation Bond (PR-ABOND)}

Sub-construct: Church

Section IV B.

\begin{tabular}{|c|c|c|c|}
\hline$\#$ & Statement & Response & \\
\hline 1 & At church I am the & $\begin{array}{l}\text { Audience } \\
\text { Coding Value }\end{array}$ & $\begin{array}{llllllllll}7 & 6 & 5 & 4 & 3 & 4 & 5 & 6 & 7 \\
1 & 2 & 3 & 4 & 5 & 6 & 7 & 8 & 9\end{array}$ \\
\hline 5 & At church I am the & $\begin{array}{l}\text { Outsider } \\
\text { Coding Value }\end{array}$ & $\begin{array}{lllllllll}7 & 6 & 5 & 4 & 3 & 4 & 5 & 6 & 7 \\
1 & 2 & 3 & 4 & 5 & 6 & 7 & 8 & 9\end{array}$ \\
\hline 9 & Church people are & $\begin{array}{l}\text { Not like me } \\
\text { Coding Value }\end{array}$ & $\begin{array}{llllllllll}7 & 6 & 5 & 4 & 3 & 4 & 5 & 6 & 7 \\
1 & 2 & 3 & 4 & 5 & 6 & 7 & 8 & 9\end{array}$ \\
\hline 13 & At church I am & $\begin{array}{l}\text { Unneeded } \\
\text { Coding Value }\end{array}$ & $\begin{array}{llllllllll}7 & 6 & 5 & 4 & 3 & 4 & 5 & 6 & 7 \\
1 & 2 & 3 & 4 & 5 & 6 & 7 & 8 & 9\end{array}$ \\
\hline 17 & At church I know more & $\begin{array}{l}\text { Acquaintances } \\
\text { Coding Value }\end{array}$ & $\begin{array}{llllllllll}7 & 6 & 5 & 4 & 3 & 4 & 5 & 6 & 7 \\
1 & 2 & 3 & 4 & 5 & 6 & 7 & 8 & 9\end{array}$ \\
\hline
\end{tabular}

Sub-construct: School

Section IV B.

\# Statement Response

2 In school activities I Audience 76654344567 Participant am the Coding Value 1234566789

6 In school activities I Outsider $\quad \begin{array}{lllllllll}7 & 6 & 5 & 4 & 3 & 4 & 5 & 6 & 7\end{array}$ Insider am the Coding Value 1234566789

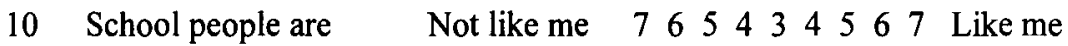
Coding Value 12234456788

14 In school activities I Unneeded 76654434567 Needed

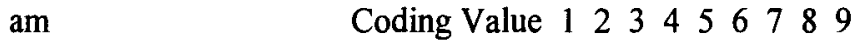

18 At school I know Acquaintances 765434567 Close Friends more Coding Value 1234456789

Note. The numeric codes for each continuum statement are listed directly below the numbered response, from $7=1$ to $7=9$. 


\section{Parent Religiosity: Affiliation Bond (PR-ABOND)}

\section{Sub-construct: Social Groups}

Section IV A.

\# $\quad$ Statement

3 In social groups I am the

7 In social groups I am the

11 Social group people are

15 In social groups I am

19 In social groups I know more
Response

Audience $\quad 765434567$ Participant Coding Value 12234556789

Outsider $\quad 765434567$ Insider Coding Value 11233456767

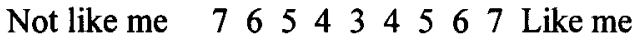
Coding Value 12234456789

Unneeded $\quad 765434567$ Needed Coding Value 11234456787

Acquaintances 7665434567 Close Friends Coding Value 11234556789

\section{Sub-construct: Civic Groups Section IV B.}

\# Statement

$4 \quad$ In civic groups I am the

8 In civic groups I am the

12 Civic group people are

16 In civic groups I am

20 In civic groups I know more
Response:

Audience $\quad \begin{array}{lllllllll}7 & 6543456 & & \\ & \end{array}$ Coding Value 122345567889

Outsider $\quad 765434567$ Insider Coding Value 12234456789

Not like me $\begin{array}{llllllllll}7 & 6 & 5 & 4 & 4 & 5 & 6 & 7 \text { Like me }\end{array}$ Coding Value 112344567889

Unneeded $\quad 7655434567$ Needed Coding Value 12234456789

Acquaintances $76 \begin{array}{llllllll}6 & 4 & 3 & 4 & 5 & 7 & \text { Close Friends }\end{array}$ Coding Value 12234556789

Note. The numeric codes for each continuum statement are listed directly below the numbered response, from $7=1$ to $7=9$. 


\section{APPENDIX S}

Table S1

Variable Operational Definitions by Type of Variable

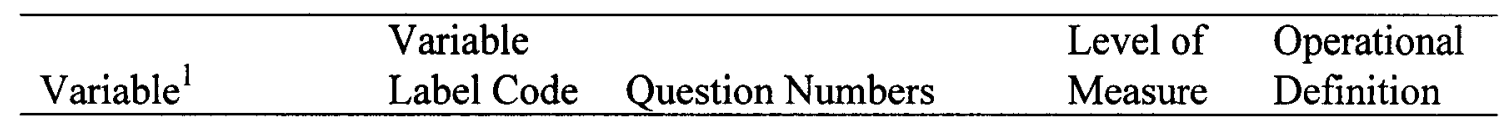

Demographic Controls

\begin{tabular}{|c|c|c|c|}
\hline $\begin{array}{l}\text { Socioeconomic } \\
\text { Status }\end{array}$ & SES & $\begin{array}{l}\text { Derived from } \\
\text { Parent V A } 9 \text { and V } \\
\text { C. } 1-6\end{array}$ & Ratio \\
\hline
\end{tabular}

Family Structure FS Derived from $\quad$ Ratio In Appendix

Parent V A. 3, 6, 7 T, Reverse

score

Special Education $\quad$ SDI $\quad$ Student V.5 Nominal $1=$ not SDI

$0=\mathrm{SDI}$

\section{Parent Rural Values}

\begin{tabular}{|c|c|c|c|c|}
\hline $\begin{array}{l}\text { Anti- } \\
\text { Intellectualism }\end{array}$ & RV-AI & $\begin{array}{l}\text { II. } 1,2,4 \text { (Reversed) } \\
\text { II. } 3,8,9,29\end{array}$ & Interval & $\begin{aligned} 5= & \text { Strongly } \\
& \text { Agree } \\
4= & \text { Agree } \\
3= & \text { Neutral } \\
2= & \text { Disagree } \\
1= & \text { Strongly } \\
& \text { Disagree }\end{aligned}$ \\
\hline
\end{tabular}

Social

RV-SC/T II. 5-7, 20-22
Interval $\quad 5=$ Strongly
Agree
$4=$ Agree
$3=$ Neutral
$2=$ Disagree
$1=$ Strongly
Disagree

(continued) 


\begin{tabular}{|c|c|c|c|c|}
\hline Variable $^{1}$ & $\begin{array}{l}\text { Variable } \\
\text { Label Code }\end{array}$ & Question Numbers & $\begin{array}{l}\text { Level of } \\
\text { Measure }\end{array}$ & $\begin{array}{l}\text { Operational } \\
\text { Definition }\end{array}$ \\
\hline
\end{tabular}

$\begin{array}{ccc}\text { Ethnocentricity } & \text { RV-E II. 10-13,16,19 Interval } & 5=\text { Strongly } \\ & \text { Agree } \\ 4 & =\text { Agree } \\ 3 & =\text { Neutral } \\ 2 & =\text { Disagree } \\ 1 & =\text { Strongly } \\ & \text { Disagree }\end{array}$

Stewardship of RV-SE Earth

$$
\text { Earth }
$$

Good Old Boy RV-GOB
II. $14,15,17,18, \quad$ Interval 30

$$
\begin{aligned}
5= & \text { Strongly } \\
& \text { Agree } \\
4= & \text { Agree } \\
3= & \text { Neutral } \\
2= & \text { Disagree } \\
1= & \text { Strongly } \\
& \text { Disagree }
\end{aligned}
$$

Interval $\quad 5=$ Strongly

Agree

$4=$ Agree

$3=$ Neutral

$2=$ Disagree

$1=$ Strongly

Disagree

Parent Religiosity Values

$\begin{array}{rcrl}\text { Religious Belief PR-BEL III A. 1-13, 20-22 Interval } & 5=\text { Strongly } \\ & \text { Agree } \\ 4 & =\text { Agree } \\ 3 & =\text { Neutral } \\ 2 & =\text { Disagree } \\ 1 & =\text { Strongly } \\ & \text { Disagree }\end{array}$




\begin{tabular}{|c|c|c|c|c|}
\hline Variable $^{1}$ & $\begin{array}{l}\text { Variable } \\
\text { Label Code }\end{array}$ & Question Numbers & $\begin{array}{l}\text { Level of } \\
\text { Measure }\end{array}$ & $\begin{array}{l}\text { Operational } \\
\text { Definition }\end{array}$ \\
\hline
\end{tabular}

Religious Ritual PR-RIT

III B. 1-11

$$
\text { Interval } \quad \begin{aligned}
1= & \text { seldom } \\
2 & =\text { monthly } \\
3= & 2 \text { or } 3 \\
& \text { times month } \\
4= & \text { weekly } \\
5= & 2 \text { or } 3 \text { times } \\
& \text { week } \\
6= & \text { daily } \\
7= & \text { more than } \\
& \quad \text { once daily }
\end{aligned}
$$

Religious

Experience

PR-EXP

III A. 14-19, 20-22

Interval

$5=$ Strongly

Agree

$4=$ Agree

$3=$ Neutral

$2=$ Disagree

1 = Strongly

Disagree

Primary

Affiliation

PR-PAFFCH IV 1A-17A

Church

Interval $\quad \begin{aligned} 1= & \text { no chance } \\ & \text { choosing } \\ & \text { this group } \\ 2= & \text { some chance } \\ & \text { choosing } \\ & \text { this group } \\ 3= & \text { neutral } \\ 4= & \text { good chance } \\ & \text { choosing } \\ & \text { this group } \\ 5= & \text { excellent } \\ & \text { chance } \\ & \text { choosing } \\ & \text { this group }\end{aligned}$




\begin{tabular}{|c|c|c|c|c|}
\hline Variable $^{1}$ & $\begin{array}{l}\text { Variable } \\
\text { Label Code }\end{array}$ & Question Numbers & $\begin{array}{l}\text { Level of } \\
\text { Measure }\end{array}$ & $\begin{array}{l}\text { Operational } \\
\text { Definition }\end{array}$ \\
\hline $\begin{array}{l}\text { Primary } \\
\text { Affiliation } \\
\text { School }\end{array}$ & PR-PAFFSC & IV. $1 \mathrm{C}-17 \mathrm{C}$ & Interval & 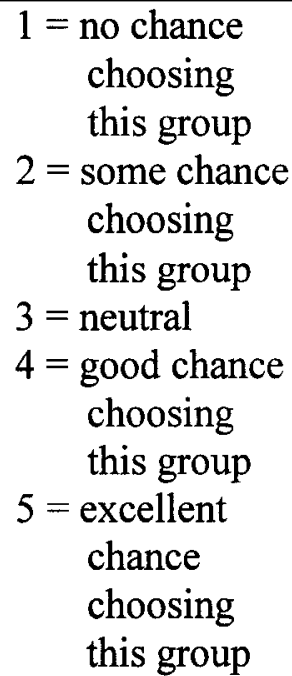 \\
\hline
\end{tabular}

Primary

Affiliation

PR-PAFFO IV. 1B-17B

Interval $1=$ no chance choosing

Other this group

$2=$ some chance choosing this group

$3=$ neutral

$4=$ good chance choosing this group

$5=$ excellent chance choosing this group

Affiliation Bond PRChurch ABONDC

IV B. $1,5,9,13,17$ Interval $1=$ Audience 7

$2=$ Audience 6

3 = Audience 5

$4=$ Audience 4

$5=$ Neutral

$6=$ Participant 4

$7=$ Participant 5

$8=$ Participant 6

$9=$ Participant 7

(continued) 


\begin{tabular}{|c|c|c|c|c|}
\hline Variable $^{1}$ & $\begin{array}{l}\text { Variable } \\
\text { Label Code }\end{array}$ & Question Numbers & $\begin{array}{l}\text { Level of } \\
\text { Measure }\end{array}$ & $\begin{array}{l}\text { Operational } \\
\text { Definition }\end{array}$ \\
\hline
\end{tabular}

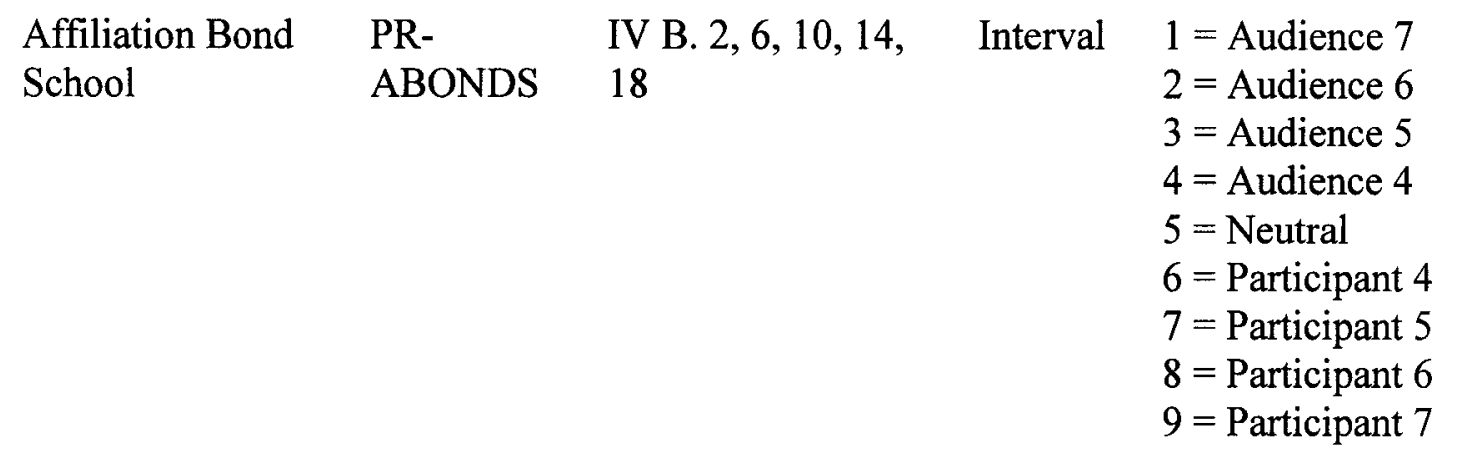

Affiliation Bond Civic

PRABONDCI

IV B. $4,8,12,16$, 20
$1=$ Audience 7

$2=$ Audience 6

$3=$ Audience 5

$4=$ Audience 4

$5=$ Neutral

$6=$ Participant 4

$7=$ Participant 5

$8=$ Participant 6

$9=$ Participant 7
Affiliation Bond Social
PR-

ABONDSO
IV B. $3,7,11,15$,

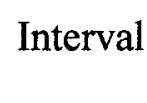

$1=$ Audience 7

$2=$ Audience 6

$3=$ Audience 5

$4=$ Audience 4

$5=$ Neutral

$6=$ Participant 4

$7=$ Participant 5

$8=$ Participant 6

$9=$ Participant 7 


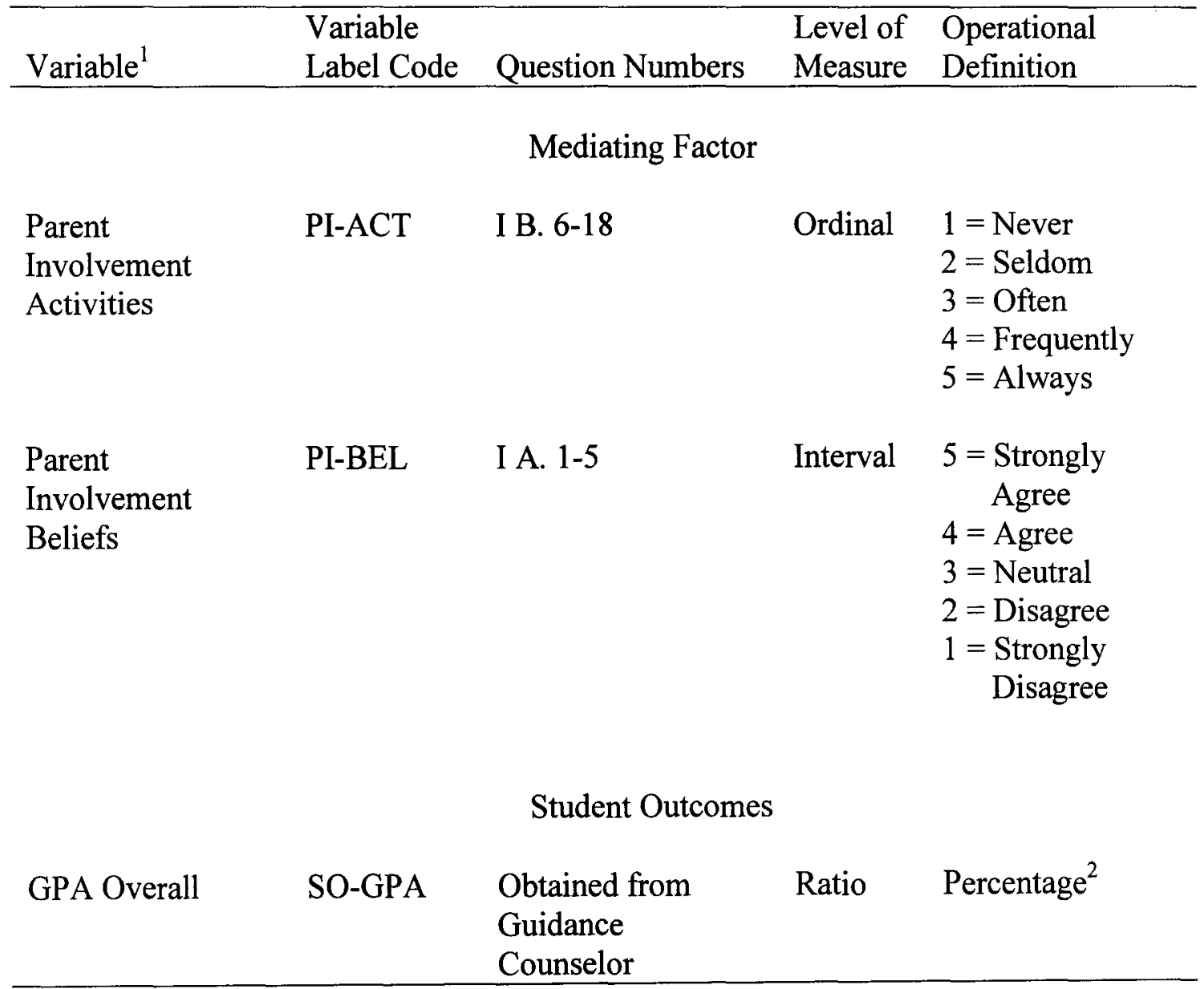

${ }^{1}$ For all scales in this appendix, the parent version and student version of the construct are identical. The only difference is who filled out the survey: parent or student.

${ }^{2}$ Each of the four subjects (English, Math, Science, Social Studies) is graded on a percentage scale. The overall GPA is the mean across the four subjects. 
Table T1

Reliability Analysis for Independent Variable: Rural Values-Anti-Intellectualism $(N=38)$

\begin{tabular}{|c|c|c|c|c|c|c|c|c|c|}
\hline \multirow[b]{2}{*}{$\begin{array}{l}\text { Survey } \\
\text { item }\end{array}$} & \multicolumn{3}{|c|}{ Parent (alpha $=.424)$} & \multicolumn{3}{|c|}{ Student (alpha $=.630$ ) } & \multicolumn{3}{|c|}{ Combined (alpha $=.635)$} \\
\hline & $M$ & $S D$ & $\begin{array}{l}\text { alpha if } \\
\text { deleted }\end{array}$ & $M$ & $S D$ & $\begin{array}{l}\text { alpha if } \\
\text { deleted }\end{array}$ & $M$ & $S D$ & $\begin{array}{l}\text { alpha if } \\
\text { deleted }\end{array}$ \\
\hline II. 1 & 2.18 & .95 & .373 & 2.76 & 1.36 & .555 & 2.47 & .90 & .561 \\
\hline II. 2 & 1.66 & .75 & .441 & 2.74 & 1.35 & .528 & 2.20 & .86 & .588 \\
\hline II. 3 & 3.63 & 1.22 & .443 & 3.92 & 1.02 & .725 & 3.78 & .89 & .714 \\
\hline II. 4 & 2.92 & .91 & .374 & 3.32 & 1.07 & .585 & 3.12 & .72 & .620 \\
\hline II. 8 & 3.97 & 1.13 & .300 & 3.29 & 1.29 & .674 & 3.63 & .93 & .605 \\
\hline II. 9 & 2.68 & 1.45 & .416 & 2.97 & 1.42 & .554 & 2.83 & 1.18 & .560 \\
\hline II. 29 & 1.87 & 1.02 & .345 & 3.32 & 1.36 & .419 & 2.59 & 1.00 & .478 \\
\hline Total & 2.70 & .87 & & 3.19 & .41 & & 2.95 & .59 & \\
\hline
\end{tabular}


Table T2

Reliability Analysis for Independent Variable: Rural Values-Social Conservatism/Traditionalism $(N=38)$

\begin{tabular}{|c|c|c|c|c|c|c|c|c|c|}
\hline \multirow[b]{2}{*}{$\begin{array}{l}\text { Survey } \\
\text { item }\end{array}$} & \multicolumn{3}{|c|}{ Parent (alpha $=.655)$} & \multicolumn{3}{|c|}{ Student (alpha $=.072)$} & \multicolumn{3}{|c|}{ Combined (alpha $=.527)$} \\
\hline & $M$ & $S D$ & $\begin{array}{l}\text { alpha if } \\
\text { deleted }\end{array}$ & $M$ & $S D$ & $\begin{array}{l}\text { alpha if } \\
\text { deleted }\end{array}$ & $M$ & $S D$ & $\begin{array}{l}\text { alpha if } \\
\text { deleted }\end{array}$ \\
\hline II. 5 & 4.32 & 1.07 & .651 & 4.34 & .94 & -.015 & 4.33 & .72 & .488 \\
\hline II. 6 & 2.71 & 1.31 & .582 & 2.05 & .98 & .172 & 2.38 & .87 & .482 \\
\hline II. 7 & 3.58 & 1.54 & .615 & 2.45 & 1.31 & .018 & 3.01 & 1.18 & .296 \\
\hline II. 20 & 2.03 & 1.22 & .600 & 2.00 & 1.14 & .053 & 3.13 & .78 & .471 \\
\hline II. 21 & 2.76 & 1.17 & .551 & 2.89 & 1.25 & .017 & 2.83 & .97 & .497 \\
\hline II. 22 & 1.26 & .75 & .659 & 1.84 & 1.22 & .097 & 1.55 & .80 & .578 \\
\hline Total & 2.78 & 1.08 & & 2.60 & .94 & & 2.87 & .92 & \\
\hline
\end{tabular}


Table T3

Reliability Analysis for Independent Variable: Rural Values-Ethnocentricity $(N=38)$

\begin{tabular}{|c|c|c|c|c|c|c|c|c|c|}
\hline \multirow[b]{2}{*}{$\begin{array}{l}\text { Survey } \\
\text { item }\end{array}$} & \multicolumn{3}{|c|}{ Parent (alpha $=.635$ ) } & \multicolumn{3}{|c|}{ Student (alpha $=.438)$} & \multicolumn{3}{|c|}{ Combined (alpha $=.571)$} \\
\hline & $M$ & $S D$ & $\begin{array}{l}\text { alpha if } \\
\text { deleted }\end{array}$ & $M$ & $S D$ & $\begin{array}{l}\text { alpha if } \\
\text { deleted }\end{array}$ & $M$ & $S D$ & $\begin{array}{l}\text { alpha if } \\
\text { deleted }\end{array}$ \\
\hline II. 10 & 4.03 & .97 & .557 & 3.63 & .97 & .422 & 3.83 & .69 & .521 \\
\hline II. 11 & 4.68 & .62 & .547 & 3.87 & 1.04 & .321 & 4.28 & .63 & .471 \\
\hline II. 12 & 2.89 & 1.09 & .593 & 3.32 & 1.23 & .335 & 3.11 & .96 & .491 \\
\hline II. 13 & 4.05 & .90 & .582 & 3.74 & .98 & .409 & 3.89 & .67 & .517 \\
\hline II. 16 & 3.11 & 1.03 & .662 & 3.37 & 1.38 & .494 & 3.24 & 1.03 & .619 \\
\hline II. 19 & 4.11 & 1.03 & .602 & 3.71 & 1.04 & .368 & 3.91 & .81 & .531 \\
\hline Total & 3.81 & .68 & & 3.61 & .44 & & 3.71 & .45 & \\
\hline
\end{tabular}


Table T4

Reliability Analysis for Independeni Variable: Rural Values-Stewardship of Earth $(N=38)$

\begin{tabular}{|c|c|c|c|c|c|c|c|c|c|}
\hline \multirow[b]{2}{*}{$\begin{array}{l}\text { Survey } \\
\text { item }\end{array}$} & \multicolumn{3}{|c|}{ Parent (alpha $=.647)$} & \multicolumn{3}{|c|}{ Student (alpha $=.606)$} & \multicolumn{3}{|c|}{ Combined (alpha $=.622)$} \\
\hline & $M$ & $S D$ & $\begin{array}{l}\text { alpha if } \\
\text { deleted }\end{array}$ & $M$ & $S D$ & $\begin{array}{l}\text { alpha if } \\
\text { deleted }\end{array}$ & $M$ & $S D$ & $\begin{array}{l}\text { alpha if } \\
\text { deleted }\end{array}$ \\
\hline II. 14 & 4.34 & .87 & .510 & 4.05 & .90 & .471 & 4.20 & .55 & .497 \\
\hline II. 15 & 4.11 & .76 & .620 & 4.16 & .75 & .470 & 4.13 & .59 & .575 \\
\hline II. 17 & 4.29 & .73 & .517 & 3.89 & 1.06 & .313 & 4.09 & .71 & .411 \\
\hline II. 18 & 4.26 & 1.06 & .481 & 4.16 & 1.03 & .508 & 4.21 & .77 & .422 \\
\hline II. 30 & 2.29 & 1.45 & .815 & 2.26 & 1.11 & .816 & 2.28 & 1.03 & .837 \\
\hline Total & 3.86 & .88 & & 3.71 & .81 & & 3.78 & .84 & \\
\hline
\end{tabular}


Table T5

Reliability Analysis for Independent Variable: Rural Values-Good Old Boy $(N=38)$

\begin{tabular}{|c|c|c|c|c|c|c|c|c|c|}
\hline \multirow[b]{2}{*}{$\begin{array}{l}\text { Survey } \\
\text { item }\end{array}$} & \multicolumn{3}{|c|}{ Parent (alpha $=.376)$} & \multicolumn{3}{|c|}{ Student (alpha $=.789)$} & \multicolumn{3}{|c|}{ Combined (alpha $=.620)$} \\
\hline & $M$ & $S D$ & $\begin{array}{l}\text { alpha if } \\
\text { deleted }\end{array}$ & $M$ & $S D$ & $\begin{array}{l}\text { alpha if } \\
\text { deleted }\end{array}$ & $M$ & $S D$ & $\begin{array}{l}\text { alpha if } \\
\text { deleted }\end{array}$ \\
\hline II. 23 & 3.13 & 1.32 & .299 & 3.08 & 1.28 & .784 & 3.11 & .95 & .610 \\
\hline II. 24 & 3.32 & 1.23 & .306 & 3.61 & 1.22 & .736 & 3.46 & .86 & .570 \\
\hline II. 25 & 2.26 & 1.06 & .295 & 3.32 & 1.34 & .704 & 2.79 & 1.00 & .471 \\
\hline II. 26 & 3.42 & 1.24 & .367 & 3.21 & 1.26 & .818 & 3.32 & .96 & .681 \\
\hline II. 27 & 1.97 & 1.20 & .326 & 3.13 & 1.44 & .737 & 2.55 & .94 & .521 \\
\hline II. 28 & 1.84 & 1.15 & .403 & 2.82 & 1.50 & .744 & 2.33 & .96 & .565 \\
\hline Totals & 2.66 & .71 & & 3.19 & .26 & & 2.93 & .44 & \\
\hline
\end{tabular}


Table T6

Reliability Analysis for Independent Variable: Parent Religiosity-Belief $(N=38)$

\begin{tabular}{|c|c|c|c|c|c|c|c|c|c|}
\hline \multirow[b]{2}{*}{$\begin{array}{l}\text { Survey } \\
\text { item }\end{array}$} & \multicolumn{3}{|c|}{ Parent (alpha $=.762$ ) } & \multicolumn{3}{|c|}{ Student (alpha $=.865$ ) } & \multicolumn{3}{|c|}{ Combined (alpha $=.854)$} \\
\hline & $M$ & $S D$ & $\begin{array}{l}\text { alpha if } \\
\text { deleted }\end{array}$ & $M$ & $S D$ & $\begin{array}{l}\text { alpha if } \\
\text { deleted }\end{array}$ & $M$ & $S D$ & $\begin{array}{l}\text { alpha if } \\
\text { deleted }\end{array}$ \\
\hline IIIA. 1 & 2.47 & 1.41 & .751 & 3.39 & 1.08 & .870 & 2.93 & .90 & .854 \\
\hline IIIA. 2 & 2.47 & .86 & .759 & 2.92 & .88 & .872 & 2.70 & .54 & .860 \\
\hline IIIA. 3 & 3.26 & 1.06 & .756 & 3.18 & 1.04 & .871 & 3.22 & .72 & .855 \\
\hline IIIA. 4 & 3.06 & 1.42 & .771 & 2.34 & 1.17 & .873 & 2.68 & .92 & .855 \\
\hline IIIA. 5 & 2.29 & 1.14 & .764 & 2.63 & 1.10 & .867 & 2.46 & .76 & .862 \\
\hline IIIA. 6 & 4.34 & 1.10 & .738 & 4.13 & 1.09 & .845 & 4.24 & .88 & .831 \\
\hline IIIA. 7 & 4.18 & 1.11 & .759 & 3.58 & 1.29 & .864 & 3.88 & .83 & .853 \\
\hline IIIA. 8 & 4.53 & .83 & .737 & 3.98 & 1.13 & .847 & 4.25 & .80 & .836 \\
\hline IIIA. 9 & 4.66 & .78 & .733 & 4.08 & 1.12 & .845 & 4.37 & .83 & .829 \\
\hline IIIA. 10 & 4.58 & 1.08 & .748 & 4.63 & .88 & .849 & 4.61 & .84 & .839 \\
\hline
\end{tabular}


Table T6 (continued)

Reliability Analysis for Independent Variable: Parent Religiosity-Belief $(N=38)$

\begin{tabular}{|c|c|c|c|c|c|c|c|c|c|}
\hline \multirow[b]{2}{*}{$\begin{array}{l}\text { Survey } \\
\text { item }\end{array}$} & \multicolumn{3}{|c|}{ Parent (alpha $=.762$ ) } & \multicolumn{3}{|c|}{ Student (alpha $=.865$ ) } & \multicolumn{3}{|c|}{ Combined (alpha $=.854)$} \\
\hline & $M$ & $S D$ & $\begin{array}{l}\text { alpha if } \\
\text { deleted }\end{array}$ & $M$ & $S D$ & $\begin{array}{l}\text { alpha if } \\
\text { deleted }\end{array}$ & $M$ & $S D$ & $\begin{array}{l}\text { alpha if } \\
\text { deleted }\end{array}$ \\
\hline IIIA. 11 & 4.47 & 1.18 & .766 & 4.58 & .92 & .845 & 4.53 & .83 & .844 \\
\hline IIIA. 12 & 4.37 & 1.02 & .743 & 4.34 & 1.21 & .841 & 4.36 & .92 & .832 \\
\hline IIIA. 13 & 3.68 & 1.42 & .732 & 3.37 & 1.15 & .851 & 3.53 & 1.10 & .841 \\
\hline IIIA. 20 & 2.47 & 1.43 & .773 & 3.34 & 1.38 & .869 & 2.91 & 1.22 & .866 \\
\hline IIIA. 21 & 4.03 & 1.24 & .750 & 4.24 & 1.24 & .849 & 4.13 & .97 & .839 \\
\hline IIIA. 22 & 4.29 & 1.14 & .713 & 4.18 & 1.22 & .841 & 4.18 & .97 & .826 \\
\hline Totals & 3.70 & .88 & & 3.68 & .69 & & 3.69 & .75 & \\
\hline
\end{tabular}


Table T7

Reliability Analysis for Independent Variable: Parent Religiosity-Ritual $(N=38)$

\begin{tabular}{|c|c|c|c|c|c|c|c|c|c|}
\hline \multirow[b]{2}{*}{$\begin{array}{l}\text { Survey } \\
\text { item }\end{array}$} & \multicolumn{3}{|c|}{ Parent (alpha $=.869)$} & \multicolumn{3}{|c|}{ Student (alpha $=.909)$} & \multicolumn{3}{|c|}{ Combined (alpha $=.912)$} \\
\hline & $M$ & $S D$ & $\begin{array}{l}\text { alpha if } \\
\text { deleted }\end{array}$ & $M$ & $S D$ & $\begin{array}{l}\text { alpha if } \\
\text { deleted }\end{array}$ & $M$ & $S D$ & $\begin{array}{l}\text { alpha if } \\
\text { deleted }\end{array}$ \\
\hline IIIB. 1 & 3.37 & 1.70 & .845 & 3.51 & 1.74 & .897 & 3.41 & 1.54 & .899 \\
\hline IIIB. 2 & 2.66 & 1.76 & .857 & 3.16 & 1.76 & .896 & 2.88 & 1.34 & .900 \\
\hline IIIB. 3 & 3.18 & 1.63 & .848 & 2.95 & 1.72 & .896 & 3.04 & 1.44 & .899 \\
\hline IIIB. 4 & 3.95 & 1.99 & .848 & 3.60 & 2.14 & .902 & 3.76 & 1.55 & .901 \\
\hline IIIB. 5 & 3.95 & 1.82 & .846 & 5.49 & 1.98 & .898 & 3.68 & 1.51 & .898 \\
\hline IIIB. 6 & 4.87 & 1.96 & .853 & 4.97 & 2.23 & .898 & 4.86 & 1.74 & .902 \\
\hline IIIB. 7 & 4.00 & 2.34 & .895 & 3.41 & 2.35 & .902 & 3.70 & 1.59 & .911 \\
\hline IIIB. 8 & 5.71 & 1.68 & .848 & 5.57 & 1.92 & .898 & 5.66 & 1.50 & .900 \\
\hline IIIB. 9 & 3.34 & 1.28 & .857 & 3.03 & 1.59 & .903 & 3.20 & 1.17 & .905 \\
\hline IIIB. 10 & 2.21 & 1.73 & .873 & 1.84 & 1.46 & .909 & 2.03 & 1.35 & .914 \\
\hline
\end{tabular}


Table T7 (continued)

Reliability Analysis for Independent Variable: Parent Religiosity-Ritual $(N=38)$

\begin{tabular}{|c|c|c|c|c|c|c|c|c|c|}
\hline \multirow[b]{2}{*}{$\begin{array}{l}\text { Survey } \\
\text { item }\end{array}$} & \multicolumn{3}{|c|}{ Parent (alpha $=.869)$} & \multicolumn{3}{|c|}{ Student (alpha $=.909)$} & \multicolumn{3}{|c|}{ Combined (alpha $=.912)$} \\
\hline & $M$ & $S D$ & $\begin{array}{l}\text { alpha if } \\
\text { deleted }\end{array}$ & $M$ & $S D$ & $\begin{array}{l}\text { alpha if } \\
\text { deleted }\end{array}$ & $M$ & $S D$ & $\begin{array}{l}\text { alpha if } \\
\text { deleted }\end{array}$ \\
\hline IIIB. 11 & 1.84 & 1.33 & .865 & 1.70 & 1.20 & .913 & 1.76 & 1.00 & .911 \\
\hline Totals & 3.55 & 1.12 & & 3.38 & 1.14 & & 3.45 & 1.12 & \\
\hline
\end{tabular}

Note. Responses are based on a 7-point Likert-type scale. 
Table T8

Reliability Analysis for Independent Variable: Parent Religiosity-Experience $(N=38)$

\begin{tabular}{|c|c|c|c|c|c|c|c|c|c|}
\hline \multirow[b]{2}{*}{$\begin{array}{l}\text { Survey } \\
\text { item }\end{array}$} & \multicolumn{3}{|c|}{ Parent (alpha $=.679)$} & \multicolumn{3}{|c|}{ Student (alpha $=.819)$} & \multicolumn{3}{|c|}{ Combined (alpha $=.801)$} \\
\hline & $M$ & $S D$ & $\begin{array}{l}\text { alpha if } \\
\text { deleted }\end{array}$ & $M$ & $S D$ & $\begin{array}{l}\text { alpha if } \\
\text { deleted }\end{array}$ & $M$ & $S D$ & $\begin{array}{l}\text { alpha if } \\
\text { deleted }\end{array}$ \\
\hline IIIA. 14 & 4.16 & 1.24 & .686 & 4.13 & 1.70 & .786 & 4.14 & .92 & .786 \\
\hline IIIA. 15 & 4.37 & 1.17 & .648 & 4.35 & .99 & .775 & 4.34 & .83 & .748 \\
\hline IIIA. 16 & 4.00 & 1.12 & .522 & 3.66 & 1.15 & .771 & 3.83 & .86 & .725 \\
\hline IIIA. 17 & 4.03 & 1.22 & .551 & 4.00 & 1.21 & .804 & 4.01 & .96 & .752 \\
\hline IIIA. 18 & 4.11 & 1.13 & .566 & 3.68 & 1.23 & .765 & 3.89 & .90 & .734 \\
\hline IIIA. 19 & 2.47 & 1.57 & .779 & 2.68 & 1.51 & .837 & 2.58 & 1.23 & .863 \\
\hline Totals & 3.85 & .69 & & 3.75 & .58 & & 3.80 & .63 & \\
\hline
\end{tabular}


Table T9

Reliability Analysis for Independent Variable: Parent Religiosity-Primary Affiliation $(N=38)$

\begin{tabular}{|c|c|c|c|c|c|c|c|c|c|}
\hline \multirow[b]{2}{*}{$\begin{array}{l}\text { Survey } \\
\text { item }\end{array}$} & \multicolumn{3}{|c|}{ Parent (alpha $=.925)$} & \multicolumn{3}{|c|}{ Student (alpha $=.964)$} & \multicolumn{3}{|c|}{ Combined $($ alpha $=.962)$} \\
\hline & $M$ & $S D$ & $\begin{array}{l}\text { alpha if } \\
\text { deleted }\end{array}$ & $M$ & $S D$ & $\begin{array}{l}\text { alpha if } \\
\text { deleted }\end{array}$ & $M$ & $S D$ & $\begin{array}{l}\text { alpha if } \\
\text { deleted }\end{array}$ \\
\hline IVA. 1 & 4.11 & 1.01 & .922 & 3.47 & 1.45 & .966 & 3.79 & 1.02 & .961 \\
\hline IVA. 2 & 3.95 & 1.11 & .923 & 3.29 & 1.35 & .967 & 3.62 & .88 & .961 \\
\hline IVA. 3 & 4.08 & 1.05 & .923 & 3.16 & 1.15 & .967 & 3.62 & .88 & .961 \\
\hline IVA. 4 & 3.50 & 1.20 & .921 & 2.92 & 1.32 & .966 & 3.21 & .86 & .961 \\
\hline IVA. 5 & 4.42 & .83 & .923 & 4.03 & 1.24 & .966 & 4.22 & .79 & .961 \\
\hline IVA. 6 & 3.18 & 1.37 & .921 & 2.82 & 1.31 & .967 & 3.00 & 1.03 & .962 \\
\hline IVA. 7 & 3.34 & 1.44 & .922 & 3.47 & 1.25 & .967 & 3.41 & 1.08 & .962 \\
\hline IVA. 8 & 3.50 & 1.37 & .923 & 2.95 & 1.41 & .966 & 3.22 & 1.04 & .962 \\
\hline IVA. 9 & 3.79 & .99 & .923 & 2.84 & 1.48 & .966 & 3.32 & .92 & .962 \\
\hline IVA. 10 & 4.18 & 1.04 & .923 & 3.84 & 1.33 & .966 & 4.01 & .93 & .961 \\
\hline
\end{tabular}


Table T9 (continued)

Reliability Analysis for Independent Variable: Parent Religiosity-Primary Affiliation $(N=38)$

\begin{tabular}{|c|c|c|c|c|c|c|c|c|c|}
\hline \multirow[b]{2}{*}{$\begin{array}{l}\text { Survey } \\
\text { item }\end{array}$} & \multicolumn{3}{|c|}{ Parent (alpha $=.925)$} & \multicolumn{3}{|c|}{ Student (alpha $=.964)$} & \multicolumn{3}{|c|}{ Combined (alpha $=.962)$} \\
\hline & $M$ & $S D$ & $\begin{array}{l}\text { alpha if } \\
\text { deleted }\end{array}$ & $M$ & $S D$ & $\begin{array}{l}\text { alpha if } \\
\text { deleted }\end{array}$ & $M$ & $S D$ & $\begin{array}{l}\text { alpha if } \\
\text { deleted }\end{array}$ \\
\hline IVA. 11 & 4.39 & 1.03 & .923 & 4.21 & 1.32 & .966 & 4.30 & .843 & .962 \\
\hline IVA. 12 & 3.11 & 1.39 & .921 & 2.53 & 1.37 & .966 & 2.82 & 1.07 & .961 \\
\hline IVA. 13 & 3.00 & 1.54 & .921 & 2.79 & 1.44 & .966 & 2.89 & 1.12 & .961 \\
\hline IVA. 14 & 3.45 & 1.22 & .924 & 2.89 & 1.52 & 967 & 3.17 & 1.06 & .962 \\
\hline IVA. 15 & 4.16 & .97 & .923 & 4.26 & 1.11 & .966 & 4.21 & .79 & .961 \\
\hline IVA. 16 & 4.08 & .94 & .923 & 4.03 & 1.17 & .966 & 4.05 & .79 & .961 \\
\hline IVA. 17 & 4.16 & 1.08 & .922 & 4.00 & 1.23 & .967 & 4.08 & .89 & .962 \\
\hline IVB. 1 & 3.63 & 1.10 & .923 & 4.32 & .90 & .968 & 3.88 & .77 & .963 \\
\hline IVB. 2 & 2.16 & 1.24 & .922 & 3.16 & 1.24 & .967 & 3.16 & .91 & .962 \\
\hline IVB. 3 & 3.97 & 1.08 & .924 & 4.00 & 1.01 & .967 & 4.00 & .89 & .962 \\
\hline IVB. 4 & 3.24 & 1.22 & .922 & 3.61 & 1.55 & 9.66 & 3.42 & .90 & $\begin{array}{c}.961 \\
\text { continues }\end{array}$ \\
\hline
\end{tabular}


Table T9 (continued)

Reliability Analysis for Independent Variable: Parent Religiosity-Primary Affiliation $(N=38)$

\begin{tabular}{|c|c|c|c|c|c|c|c|c|c|}
\hline \multirow[b]{2}{*}{$\begin{array}{l}\text { Survey } \\
\text { item }\end{array}$} & \multicolumn{3}{|c|}{ Parent (alpha $=.925)$} & \multicolumn{3}{|c|}{ Student (alpha $=.964)$} & \multicolumn{3}{|c|}{ Combined (alpha $=.962)$} \\
\hline & $M$ & $S D$ & $\begin{array}{l}\text { alpha if } \\
\text { deleted }\end{array}$ & $M$ & $S D$ & $\begin{array}{l}\text { alpha if } \\
\text { deleted }\end{array}$ & $M$ & $S D$ & $\begin{array}{l}\text { alpha if } \\
\text { deleted }\end{array}$ \\
\hline IVB. 5 & 3.16 & 1.05 & .923 & 3.16 & 1.37 & .966 & 3.16 & .96 & .962 \\
\hline IVB.6 & 2.95 & 1.29 & .934 & 3.89 & 1.18 & .967 & 3.42 & .90 & .962 \\
\hline IVB. 7 & 3.50 & 1.48 & .923 & 4.26 & 1.00 & .967 & 3.88 & 1.00 & .961 \\
\hline IVB. 8 & 3.61 & 1.10 & .921 & 4.08 & 1.17 & .966 & 3.84 & .89 & .961 \\
\hline IVB. 9 & 3.53 & .98 & .925 & 4.13 & 1.32 & .966 & 3.83 & .97 & .961 \\
\hline IVB. 10 & 3.32 & 1.12 & .925 & 3.34 & 1.46 & .966 & 3.33 & .92 & .961 \\
\hline IVB. 11 & 3.32 & 1.04 & .923 & 3.92 & 1.17 & .966 & 3.62 & .87 & .961 \\
\hline IVB. 12 & 2.97 & 1.33 & .923 & 3.61 & 1.33 & .966 & 3.30 & 1.09 & .962 \\
\hline IVB. 13 & 2.58 & 1.31 & .922 & 3.03 & 1.46 & .966 & 2.81 & 1.05 & .961 \\
\hline IVB. 14 & 2.71 & 1.31 & .924 & 4.05 & 1.25 & .966 & 3.38 & .91 & .962 \\
\hline IVB. 15 & 3.55 & 1.13 & .925 & 3.71 & 1.39 & .966 & 3.63 & .96 & .961 \\
\hline
\end{tabular}


Table T9 (continued)

Reliability Analysis for Independent Variable: Parent Religiosity-Primary Affiliation $(N=38)$

\begin{tabular}{|c|c|c|c|c|c|c|c|c|c|}
\hline \multirow[b]{2}{*}{$\begin{array}{l}\text { Survey } \\
\text { item }\end{array}$} & \multicolumn{3}{|c|}{ Parent (alpha $=.925)$} & \multicolumn{3}{|c|}{ Student (alpha $=.964)$} & \multicolumn{3}{|c|}{ Combined (alpha $=.962)$} \\
\hline & $M$ & $S D$ & $\begin{array}{l}\text { alpha if } \\
\text { deleted }\end{array}$ & $M$ & $S D$ & $\begin{array}{l}\text { alpha if } \\
\text { deleted }\end{array}$ & $M$ & $S D$ & $\begin{array}{l}\text { alpha if } \\
\text { deleted }\end{array}$ \\
\hline IVB. 16 & 3.42 & 1.00 & .923 & 4.18 & 1.06 & .966 & 3.80 & .79 & .961 \\
\hline IVB. 17 & 3.13 & 1.12 & .922 & 3.63 & 1.20 & .967 & 3.38 & 1.00 & .961 \\
\hline IVC. 1 & 3.39 & 1.17 & .925 & 3.66 & 1.21 & .967 & 3.53 & .94 & .962 \\
\hline IVC. 2 & 3.55 & 1.18 & .926 & 3.34 & 1.02 & .967 & 3.45 & .78 & .963 \\
\hline IVC. 3 & 3.29 & 1.18 & .922 & 3.47 & 1.31 & .967 & 3.38 & .96 & .961 \\
\hline IVC. 4 & 3.34 & 1.21 & .926 & 3.16 & 1.42 & .966 & 3.25 & .84 & .962 \\
\hline IVC. 5 & 3.13 & 1.17 & .924 & 3.13 & 1.32 & .966 & 4.91 & 1.32 & .962 \\
\hline IVC. 6 & 3.05 & 1.29 & .924 & 3.37 & 1.40 & .967 & 3.21 & 1.01 & .963 \\
\hline IVC. 7 & 3.42 & 1.39 & .923 & 3.84 & 1.22 & .967 & 3.63 & .96 & .962 \\
\hline IVC. 8 & 3.39 & 1.42 & .923 & 3.42 & 1.41 & .966 & 3.41 & 1.18 & .961 \\
\hline IVC. 9 & 3.42 & 1.18 & .924 & 3.55 & 1.33 & .966 & 3.49 & .89 & .961 \\
\hline
\end{tabular}


Table T9 (continued)

Reliability Analysis for Independent Variable: Parent Religiosity-Primary Affiliation $(N=38)$

\begin{tabular}{|c|c|c|c|c|c|c|c|c|c|}
\hline \multirow[b]{2}{*}{$\begin{array}{l}\text { Survey } \\
\text { item }\end{array}$} & \multicolumn{3}{|c|}{ Parent (alpha $=.925)$} & \multicolumn{3}{|c|}{ Student (alpha $=.964)$} & \multicolumn{3}{|c|}{ Combined (alpha $=.962)$} \\
\hline & $M$ & $S D$ & $\begin{array}{l}\text { alpha if } \\
\text { deleted }\end{array}$ & $M$ & $S D$ & $\begin{array}{l}\text { alpha if } \\
\text { deleted }\end{array}$ & $M$ & $S D$ & $\begin{array}{l}\text { alpha if } \\
\text { deleted }\end{array}$ \\
\hline IVC. 10 & 3.34 & 1.15 & .924 & 3.42 & 1.43 & .966 & 3.38 & 1.02 & .962 \\
\hline IVC. 11 & 2.95 & 1.23 & .921 & 3.47 & 1.50 & .966 & 3.21 & 1.09 & .961 \\
\hline IVC. 12 & 3.00 & 1.39 & .923 & 2.95 & 1.31 & .967 & 2.97 & .91 & .962 \\
\hline IVC. 13 & 2.74 & 1.37 & .921 & 2.61 & 1.41 & .967 & 2.67 & .91 & .962 \\
\hline IVC. 14 & 3.26 & 1.13 & .925 & 3.26 & 1.35 & .967 & 3.26 & .82 & .962 \\
\hline IVC. 15 & 3.71 & 1.04 & .925 & 3.39 & 1.41 & .967 & 3.55 & .86 & .962 \\
\hline IVC. 16 & 3.34 & 1.24 & .923 & 3.74 & 1.35 & .966 & 3.54 & 1.05 & .962 \\
\hline IVC. 17 & 3.26 & 1.11 & .923 & 3.26 & 1.43 & .968 & 3.26 & 1.02 & .963 \\
\hline Totals & 3.45 & .43 & & 3.50 & .47 & & 3.51 & .43 & \\
\hline
\end{tabular}


Table T10

Reliability Analysis for Independent Variable: Parent Religiosity-Affiliation Bond $(N=38)$

\begin{tabular}{|c|c|c|c|c|c|c|c|c|c|}
\hline \multirow[b]{2}{*}{$\begin{array}{l}\text { Survey } \\
\text { item }\end{array}$} & \multicolumn{3}{|c|}{ Parent (alpha $=.908)$} & \multicolumn{3}{|c|}{ Student (alpha $=.916$ ) } & \multicolumn{3}{|c|}{ Combined (alpha $=.921)$} \\
\hline & $M$ & $S D$ & $\begin{array}{l}\text { alpha if } \\
\text { deleted }\end{array}$ & $M$ & $S D$ & $\begin{array}{l}\text { alpha if } \\
\text { deleted }\end{array}$ & $M$ & $S D$ & $\begin{array}{l}\text { alpha if } \\
\text { deleted }\end{array}$ \\
\hline IVB. 1 & 5.79 & 3.30 & .98 & 5.69 & 2.56 & .919 & .574 & 2.15 & .922 \\
\hline IVB. 5 & 5.79 & 2.68 & .901 & 5.74 & 2.74 & .913 & 5.76 & 2.15 & .917 \\
\hline IVB. 9 & 6.21 & 2.83 & .900 & 6.00 & 2.31 & .916 & 6.11 & 2.11 & .920 \\
\hline IVB. 13 & 6.58 & 2.61 & .899 & 6.50 & 2.39 & .914 & 6.54 & 1.94 & .917 \\
\hline IVB. 17 & 5.66 & 3.01 & .906 & 5.32 & 2.60 & .916 & 5.49 & 2.26 & .920 \\
\hline IVB. 2 & 5.97 & 2.53 & .910 & 7.05 & 1.77 & .911 & 6.51 & 1.60 & .921 \\
\hline IVB. 6 & 5.03 & 2.52 & .904 & 6.74 & 2.25 & .909 & 5.88 & 1.64 & .915 \\
\hline IVB. 10 & 5.29 & 2.42 & .901 & 5.98 & 2.69 & .912 & 5.63 & 1.88 & .915 \\
\hline IVB. 14 & 6.05 & 2.58 & .900 & 6.79 & 2.11 & .911 & 6.42 & 1.58 & .915 \\
\hline IVB. 18 & 5.16 & 2.68 & .906 & 6.71 & 2.47 & .912 & 5.93 & 1.88 & .917 \\
\hline IVB. 3 & 5.29 & 2.48 & .902 & 6.45 & 2.14 & .911 & 4.88 & 1.80 & .917 \\
\hline
\end{tabular}


Table T10 (continued)

Reliability Analysis for Independent Variable: Parent Religiosity-Affiliation Bond $(N=38)$

\begin{tabular}{|c|c|c|c|c|c|c|c|c|c|}
\hline \multirow[b]{2}{*}{$\begin{array}{l}\text { Survey } \\
\text { item }\end{array}$} & \multicolumn{3}{|c|}{ Parent (alpha $=.908)$} & \multicolumn{3}{|c|}{ Student (alpha $=.916)$} & \multicolumn{3}{|c|}{ Combined (alpha $=.921)$} \\
\hline & $M$ & $S D$ & $\begin{array}{l}\text { alpha if } \\
\text { deleted }\end{array}$ & $M$ & $S D$ & $\begin{array}{l}\text { alpha if } \\
\text { deleted }\end{array}$ & $M$ & $S D$ & $\begin{array}{l}\text { alpha if } \\
\text { deleted }\end{array}$ \\
\hline IVB. 7 & 5.68 & 2.05 & .903 & 6.68 & 2.22 & .913 & 5.39 & 1.73 & .915 \\
\hline IVB. 1 & 5.68 & 2.44 & .903 & 5.55 & 2.30 & .911 & 5.22 & 1.71 & .915 \\
\hline IVB. 15 & 5.61 & 2.18 & .899 & 5.53 & 2.23 & .909 & 5.26 & 1.79 & .913 \\
\hline IVB. 19 & 5.37 & 2.49 & .910 & 5.18 & 2.23 & .909 & 4.86 & 1.90 & .923 \\
\hline IVB. 4 & 4.21 & 2.42 & .903 & 5.71 & 2.07 & .907 & 5.87 & 1.72 & .916 \\
\hline IVB. 8 & 4.50 & 2.12 & .900 & 5.45 & 2.23 & .916 & 5.93 & 1.80 & .915 \\
\hline IVB. 12 & 5.26 & 2.19 & .902 & 6.18 & 2.37 & .908 & 5.70 & 1.93 & .916 \\
\hline IVB. 16 & 4.83 & 2.40 & .899 & 5.71 & 2.56 & .908 & 5.80 & 1.94 & .912 \\
\hline IVB. 20 & 4.26 & 2.38 & .908 & 6.00 & 2.34 & .907 & 4.86 & 1.90 & .923 \\
\hline Totals & 5.41 & .63 & & 6.05 & .56 & & 5.69 & .51 & \\
\hline
\end{tabular}

Note. Responses are based on a 9-point "Dilemma" scale. 
Table T11

Reliability Analysis for Independent Variable: Parent Involvement-Belief $(N=38)$

\begin{tabular}{|c|c|c|c|c|c|c|c|c|c|}
\hline \multirow[b]{2}{*}{$\begin{array}{l}\text { Survey } \\
\text { item }\end{array}$} & \multicolumn{3}{|c|}{ Parent (alpha $=.675)$} & \multicolumn{3}{|c|}{ Student (alpha $=.752)$} & \multicolumn{3}{|c|}{ Combined (alpha $=.735)$} \\
\hline & $M$ & $S D$ & $\begin{array}{l}\text { alpha if } \\
\text { deleted }\end{array}$ & $M$ & $S D$ & $\begin{array}{l}\text { alpha if } \\
\text { deleted }\end{array}$ & $M$ & $S D$ & $\begin{array}{l}\text { alpha if } \\
\text { deleted }\end{array}$ \\
\hline IA. 1 & 3.58 & .83 & .592 & 3.37 & 1.13 & .701 & 3.47 & .79 & .655 \\
\hline IA. 2 & 3.69 & 1.07 & .552 & 3.55 & 1.08 & .654 & 3.62 & .85 & .643 \\
\hline IA. 3 & 3.97 & 1.00 & .723 & 3.71 & 1.09 & .729 & 3.84 & .81 & .751 \\
\hline IA. 4 & 4.55 & .83 & .621 & 3.71 & 1.04 & .722 & 4.13 & .69 & .687 \\
\hline IA. 5 & 4.05 & 1.06 & .607 & 3.00 & 1.14 & .727 & 3.53 & .94 & .698 \\
\hline Totals & 3.97 & .38 & & 3.47 & .30 & & 3.72 & .27 & \\
\hline
\end{tabular}


Table T12

Reliability Analysis for Independent Variable: Parent Involvement-Action $(N=38)$

\begin{tabular}{|c|c|c|c|c|c|c|c|c|c|}
\hline \multirow[b]{2}{*}{$\begin{array}{l}\text { Survey } \\
\text { item }\end{array}$} & \multicolumn{3}{|c|}{ Parent (alpha $=.878)$} & \multicolumn{3}{|c|}{ Student $($ alpha $=.837)$} & \multicolumn{3}{|c|}{ Combined $($ alpha $=.867)$} \\
\hline & $M$ & $S D$ & $\begin{array}{l}\text { alpha if } \\
\text { deleted }\end{array}$ & $M$ & $S D$ & $\begin{array}{l}\text { alpha if } \\
\text { deleted }\end{array}$ & $M$ & $S D$ & $\begin{array}{l}\text { alpha if } \\
\text { deleted }\end{array}$ \\
\hline IB. 6 & 3.16 & 1.17 & .879 & 2.50 & .98 & .836 & 2.83 & .83 & .867 \\
\hline IB. 7 & 2.61 & 1.31 & .875 & 2.08 & 1.28 & .843 & 2.34 & .84 & .870 \\
\hline IVB. 8 & 4.21 & .99 & .875 & 3.45 & 1.52 & .828 & 3.83 & 1.03 & .870 \\
\hline IVB. 9 & 2.71 & 1.16 & .875 & 2.37 & 1.02 & .842 & 2.54 & .86 & .866 \\
\hline IVB. 10 & 2.63 & 1.17 & .861 & 2.11 & 1.01 & .837 & 2.37 & .75 & .858 \\
\hline IVB. 11 & 2.92 & 1.00 & .876 & 2.34 & 1.10 & .812 & 2.63 & .81 & .858 \\
\hline IVB. 12 & 2.47 & 1.16 & .868 & 2.42 & 1.27 & .813 & 2.45 & .95 & .847 \\
\hline IVB. 13 & 2.76 & 1.53 & .876 & 2.53 & 1.25 & .818 & 2.64 & 1.06 & .860 \\
\hline IVB. 14 & 3.42 & 1.48 & .867 & 3.37 & 1.20 & .817 & 3.29 & 1.10 & .848 \\
\hline IVB. 15 & 3.97 & 1.38 & .859 & 3.45 & 1.45 & .823 & 3.71 & 1.11 & .852 \\
\hline IVB. 16 & 3.26 & 1.25 & .861 & 3.11 & 1.25 & .820 & 3.84 & 1.03 & .844 \\
\hline
\end{tabular}


Table T12 (continued)

Reliability Analysis for Independent Variable: Parent Involvement-Action $(N=38)$

\begin{tabular}{|c|c|c|c|c|c|c|c|c|c|}
\hline \multirow[b]{2}{*}{$\begin{array}{l}\text { Survey } \\
\text { item }\end{array}$} & \multicolumn{3}{|c|}{ Parent (alpha $=.878)$} & \multicolumn{3}{|c|}{ Student (alpha $=.837$ ) } & \multicolumn{3}{|c|}{ Combined (alpha $=.867)$} \\
\hline & $M$ & $S D$ & $\begin{array}{l}\text { alpha if } \\
\text { deleted }\end{array}$ & $M$ & $S D$ & $\begin{array}{l}\text { alpha if } \\
\text { deleted }\end{array}$ & $M$ & $S D$ & $\begin{array}{l}\text { alpha if } \\
\text { deleted }\end{array}$ \\
\hline IVB. 17 & 3.58 & 1.33 & .855 & 3.16 & 1.55 & .818 & 3.37 & 1.16 & .848 \\
\hline IVB. 18 & 4.03 & 1.13 & .861 & 3.32 & 1.38 & .821 & 3.67 & .93 & .851 \\
\hline Totals & 3.21 & .59 & & 2.78 & .53 & & 3.00 & .55 & \\
\hline
\end{tabular}


Table T13

Secondary Reliability Aralysis for Independent Variable: Rural Values-Anti-Intellectualism $(N=38)$

\begin{tabular}{|c|c|c|c|c|c|c|c|c|c|}
\hline \multirow[b]{2}{*}{$\begin{array}{l}\text { Survey } \\
\text { item }\end{array}$} & \multicolumn{3}{|c|}{ Parent (alpha $=.443)$} & \multicolumn{3}{|c|}{ Student $($ alpha $=.725)$} & \multicolumn{3}{|c|}{ Combined (alpha $=.714)$} \\
\hline & $M$ & $S D$ & $\begin{array}{l}\text { alpha if } \\
\text { deleted }\end{array}$ & $M$ & $S D$ & $\begin{array}{l}\text { alpha if } \\
\text { deleted }\end{array}$ & $M$ & $S D$ & $\begin{array}{l}\text { alpha if } \\
\text { deleted }\end{array}$ \\
\hline II. 1 & 2.18 & .95 & .383 & 2.76 & 1.36 & .677 & 2.47 & .90 & .673 \\
\hline II. 2 & 1.66 & .75 & .419 & 2.74 & 1.35 & .645 & 2.20 & .86 & .667 \\
\hline II. 4 & 2.92 & .91 & .369 & 3.32 & 1.07 & .701 & 3.12 & .72 & .704 \\
\hline II. 8 & 3.97 & 1.13 & .382 & 3.29 & 1.29 & .791 & 3.63 & .93 & .726 \\
\hline II. 9 & 2.68 & 1.45 & .434 & 2.97 & 1.42 & .679 & 2.83 & 1.18 & .652 \\
\hline II. 29 & 1.87 & 1.02 & .406 & 3.32 & 1.36 & .585 & 2.59 & 1.00 & .611 \\
\hline Total & 2.55 & .85 & & 3.07 & .28 & & 2.81 & .51 & \\
\hline
\end{tabular}


Table T14

Secondary Reliability Analysis for Independent Variable: Rural Values-Ethnocentricity $(N=38)$

\begin{tabular}{|c|c|c|c|c|c|c|c|c|c|}
\hline \multirow[b]{2}{*}{$\begin{array}{l}\text { Survey } \\
\text { item }\end{array}$} & \multicolumn{3}{|c|}{ Parent (alpha $=.662)$} & \multicolumn{3}{|c|}{ Student (alpha $=.494)$} & \multicolumn{3}{|c|}{ Combined (alpha $=.619)$} \\
\hline & $M$ & $S D$ & $\begin{array}{l}\text { alpha if } \\
\text { deleted }\end{array}$ & $M$ & $S D$ & $\begin{array}{l}\text { alpha if } \\
\text { deleted }\end{array}$ & $M$ & $S D$ & $\begin{array}{l}\text { alpha if } \\
\text { deleted }\end{array}$ \\
\hline II. 10 & 4.03 & .97 & .560 & 3.63 & .97 & .462 & 3.83 & .69 & .538 \\
\hline II. 11 & 4.68 & .62 & .571 & 3.87 & 1.04 & .312 & 4.28 & .63 & .506 \\
\hline II. 12 & 2.89 & 1.09 & .658 & 3.32 & 1.23 & .438 & 3.11 & .96 & .567 \\
\hline II. 13 & 4.05 & .90 & .579 & 3.74 & .98 & .480 & 3.89 & .67 & .554 \\
\hline II. 19 & 4.11 & 1.03 & .681 & 3.71 & 1.04 & .476 & 3.91 & .81 & .656 \\
\hline Total & 4.00 & .65 & & 3.65 & .21 & & 3.80 & .43 & \\
\hline
\end{tabular}


Table T15

Secondary Reliability Analysis for Independent Variable: Rural Values-Stewardship of Earth $(N=38)$

\begin{tabular}{|c|c|c|c|c|c|c|c|c|c|}
\hline \multirow[b]{2}{*}{$\begin{array}{l}\text { Survey } \\
\text { item }\end{array}$} & \multicolumn{3}{|c|}{ Parent (alpha $=.811)$} & \multicolumn{3}{|c|}{ Student (alpha $=.816)$} & \multicolumn{3}{|c|}{ Combined (alpha $=.837)$} \\
\hline & $M$ & $S D$ & $\begin{array}{l}\text { alpha if } \\
\text { deleted }\end{array}$ & $M$ & $S D$ & $\begin{array}{l}\text { alpha if } \\
\text { deleted }\end{array}$ & $M$ & $S D$ & $\begin{array}{l}\text { alpha if } \\
\text { deleted }\end{array}$ \\
\hline II. 14 & 4.34 & .87 & 699 & 4.05 & .90 & .755 & 4.20 & .55 & .770 \\
\hline II. 15 & 4.11 & .76 & .883 & 4.16 & .75 & .778 & 4.13 & .59 & .837 \\
\hline II. 17 & 4.29 & .73 & .718 & 3.89 & 1.06 & .691 & 4.09 & .71 & .736 \\
\hline II. 18 & 4.26 & 1.06 & .704 & 4.16 & 1.03 & .818 & 4.21 & .77 & .823 \\
\hline Total & 4.07 & .12 & & 4.23 & .10 & & 4.16 & .05 & \\
\hline
\end{tabular}


Table T16

Reliability Analysis for Overall Parent Values and Parent Involvement Constructs $(N=$ 38).

Scale $\quad M \quad S D \quad$ Alpha if item deleted

$$
\text { Rural Values (alpha }=.472)
$$

Anti-Intellectualism

2.93

.46

Social Conservatism/Traditionalism

2.78

.73

.362

Ethnocentricity

3.95

.61

.482

Stewardship of Earth

4.15

.56

.336

Good Old Boy

3.19

.937

.567

Parent Religiosity (alpha $=.777$ )

\begin{tabular}{lccc} 
Belief & 3.61 & .49 & .750 \\
Ritual & 3.45 & 1.05 & .736 \\
Experience & 3.80 & .68 & .701 \\
Primary Affiliation & 3.51 & .56 & .721 \\
Affiliation Bond & 5.69 & 1.19 & .777 \\
\hline & & & (table continues)
\end{tabular}


Table 16 (continued)

Reliability Analysis for Overall Parent Values and Parent Involvement Constructs $(N=$ 38).

Scale $M$ $S D$ Alpha if item deleted

$$
\text { Parent Involvement }(\text { alpha }=.751)
$$

Belief

3.72

.57

Action

3.00

.60

Note. Under Rural Values, Social Conservatism/Traditionalism and Ethnocentricity are

Parent data; Good Old Boy is Student data. All others are Combined data.

${ }^{a}$ Ritual is based on a 7-point scale.

${ }^{\mathrm{b}}$ Affiliation Bond is based on a 9-point scale. 


\section{APPENDIX U}

\section{Parent Interview Questions}

1. Tell me about DEMS.

2. Describe the teachers and the principal at DEMS.

3. Tell me about the curricula.

4. Describe extra-curricular activities.

5. How do parents, school, and/or community interact?

6. How would you describe the atmosphere at DEMS?

7. How would you describe the quality of education at DEMS?

8. Talk about how students are treated at DEMS.

9. How do you feel your child is treated?

10. Tell me about how you feel when you visit the school.

11. How do you feel about religion and school?

12 . How would you describe your community?

13. Tell me about your activities outside school.

Researcher Checklist

1. How tolerant/supportive is DEMS of religious beliefs and values?

2. Describe how the school has responded to requests for returning prayer and the Ten Commandments to the school.

3. How does the school view/handle the teaching of evolution?

4. How does the school view/handle the teaching of Creationism?

5. In what ways have you been involved as a parent at the school?

6. In what ways have you been involved as a parent in your child's education at home?

7. In what ways do you think rural curriculum should differ from urban curriculum?

8. Describe any awareness you have of the "good old boy culture" in the DEMS system.

9. Describe any effect you or your child has had as a result of the "good old boy culture" at DEMS.

10. How does the quality of the rural education at DEMS differ from the education received by a child in a large city school?

11. How well do the elements of parent, school, community, and student overlap in the DEMS system.

12. To what degree are parents and students involved in designing the educational opportunities afforded by DEMS?

13. Describe how welcoming the school is when a parent visits.

14. Describe how highly you feel the school staff values your child.

15. Describe how concerned you feel the school staff is with your child's success.

16. Evaluate or describe your own interaction with DEMS staff/personnel.

17. Evaluate or describe your own interaction with the current principal of DEMS.

18. In what ways should DEMS honor a child's rural heritage?

19. Compare the emphasis on academics to the emphasis on sports at DEMS.

20. Describe some of the best elements of DEMS. 
21. Describe some of the elements at DEMS that you would like to see improved.

22. Describe your participation in civic and/or social organizations.

23. Which organization, from school groups, civic groups, social groups, and church groups, offers you the greatest opportunity for personal satisfaction?

24 . With which group do you find yourself spending more time?

25 . From which group do you seem to select your closest friends?

\section{DEMS Student Interview Questions}

1. Tell me about DEMS.

2. Describe the teachers and the principal at DEMS.

3. Tell me about the curricula.

4. Describe extra-curricular activities.

5. How do parents, school, and/or community interact?

6. How would you describe the atmosphere at DEMS?

7. Tell me about how would you feel if your parent visited the school.

8. Talk about how students are treated at DEMS.

9. Tell me about you and school.

10. How do you feel about religion and school?

11 . How would you describe your community?

12. Describe similarities or differences between your school and your community.

13. Tell me about your parents' activities outside school.

14. What people, other than family, do your parents' seem to spend the most time with?

\section{Researcher Checklist}

1. How well do the teachers and staff at DEMS support your religious beliefs and values?

2. Describe how the school has responded to student suggestions for returning prayer and the Ten Commandments to the school.

3. How does the school view/handle the teaching of evolution?

4. How does the school view/handle the teaching of Creationism?

5. In what ways have your parents been involved at the school?

6. In what ways have your parents been involved in your education at home?

7. In what ways do you think rural schools should differ from urban schools?

8. Describe any awareness you have of the boys being treated better than girls in the DEMS system.

9. Describe any time you have felt like you were treated as less because you were a boy or girl at DEMS.

10. How does the quality of the rural education at DEMS differ from the education received by a child in a large city school?

11. How well do parents, school, community, and students communicate with each other in the DEMS system?

12. To what degree are parents and students involved in designing the educational opportunities at DEMS?

13. Describe how welcoming the school is when a parent visits. 
14. Describe how highly valued by the school you feel.

15. Describe how concerned the school staff is with your success.

16. Evaluate or describe how the DEMS teachers treat you compared to how they treat other students.

17. Evaluate or describe how you get along with the current principal of DEMS.

18. In what ways should DEMS teach things that honor your rural background?

19. Compare the emphasis on academics to the emphasis on sports at DEMS.

20. Describe some of the best things about DEMS.

21. Describe some of the things at DEMS that you would like to see changed.

22. Describe your parents' participation in civic and/or social organizations.

23. Which organization, from school groups, civic groups, social groups, and church groups, causes your parents' to feel more like they have accomplished something?

24 . Which group do your parents spend more time with?

25 . From which group do your parents seem to select their closest friends?

\section{Faculty Interview Questions}

1. Describe your position with DEMS.

2. How do you feel about religion and school?

3. Tell me about teacher and parent interaction at DEMS.

4. Tell me about parent involvement at DEMS.

5. Describe the environment at DEMS.

\section{Researcher Checklist}

1. Describe the relationships between faculty, staff, and principal.

2. How do you feel about religious issues ate DEMS?

3. How does DEMS encourage parents to be involved?

4. Describe student attitudes toward school here at DEMS.

5. Describe parent attitudes about school at DEMS.

6. Describe the interaction between the principal and the staff at DEMS.

7. Describe how the staff of DEMS feel about changes of education reform.

8. Explain the best part of working at DEMS.

9. Explain the worst part of working at DEMS.

\section{Withdrawn Student Interview Questions}

1. Why do you think your parents withdrew you from DEMS?

2. Why do you think they brought you back to DEMS?

3. How would you compare the two schools?

4. How do you think your parents compare the two schools?

5. What do you think about prayer in schools?

6. What do you think about the Ten Commandments posted in the classroom?

Researcher Checklist

1. Why did you choose to leave Dixon Elementary School?

2. Where did you attend school upon leaving DEMS?

3. How does the academic program there compare to the one at DEMS?

(continued) 
4. How does parent involvement there compare to that at DEMS?

5. How does the quality of education there compare to that of DEMS?

6. How did your religious values influence the decision to leave DEMS?

7. What caused you to decide to return to DEMS?

8. In what ways are your parents involved in your education?

9. In what ways do you think DEMS supports or threatens your values?

10. What is your position on returning the Ten Commandments and prayer to the classroom?

\section{Support Staff Interview Questions}

1. Describe the Dixon community.

2. Tell me about the differences and/or similarities of your congregation in Louisville and the congregation here.

3. Describe your involvement with the Webster County School System.

4. How do you feel about religion and school?

5. Talk about parent, school, and community interaction.

6. Tell me about your congregation's involvement with the community.

7. What "signs" of the presence of the Holy Spirit have you noticed in your congregation?

\section{Researcher Checklist}

1. When you came here from Louisville, 7 years ago, what was your first impression of the Dixon community? How has that impression changed?

2. How would you compare the small community values to those of your urban community?

3. How would you compare the congregations of your church there to the one of Dixon First Baptist Church?

4. Describe your position with Webster County Board of Education.

5. Based on your interaction with the DEMS staff, what impressions have you of their receptiveness to parent/community involvement in the school?

6. Describe your evaluation of the Middle School.

7. Describe your involvement with DEMS students.

8. How do parents in your church, who have children in the DEMS middle school, seem to feel about the school as a support or threat to their family and religious values?

9. How traditional are the members of your church?

10. In what kinds of activities are most of your members involved within the church community?

11. Describe how involved they are in addressing the social ills of the community.

12. In what ways have you sensed the presence of the Holy Spirit in people's lives in your church?

13. Have you witnessed speaking in tongues, slaying in the spirit, jerking, "Holy" Laughter, etc.? 
14. In what ways have you witnessed the presence of the Spirit in other churches in the Dixon Community?

15. What is the size of your membership and what percent of the members seem to have a close bond with other members of the church?

16. In what ways do church members mingle and interact with each other during nonchurch time?

17. What community civic, social, or school groups have large memberships in the Dixon Community?

18. How do those organizations support or interfere with the bonding of church members or with church growth?

19. What do you see as the church's role in educating community children?

20. How has DEMS supported or interfered with church activities or church values?

\section{Custodian Interview Questions}

1. Describe your position with DEMS.

2. How do you feel about religion and schools?

3. Tell me about school and parent interaction at DEMS.

4. Tell me about parent involvement at DEMS.

5. Describe your feelings about DEMS when your children were students here.

6. Describe the environment at DEMS.

Researcher Checklist

1. Describe the relationships between faculty, staff, and principal.

2. How do you feel about religious issues at DEMS?

3. How does DEMS encourage parents to be involved?

4. Describe student attitudes toward school here at DEMS.

5. Describe parent attitudes about school at DEMS.

6. Describe the interaction between the principal and staff at DEMS.

7. Describe how the staff of DEMS feel about changes of education reform.

8. Explain the best part of working at DEMS.

9. Explain the worst part of working at DEMS. 
Sent: Friday, June 08, 2001 11:46 AM

Subject: Arkansas Love Poem

\section{AN ARKANSAS LUV POEM}

Suzy Lee fell in love.

She planned to marry Joe.

She was so happy about it all, She told her pappy so.

Pappy told her, "Suzy Gal"

You'll have to find another.

Id just as soon yo maw donut know, But Joe is yo half brother.

So Suzy forgot about her Joe And planned to marry Will, But after telling pappy this, He said, "There's trouble still."

You cant marry Will, my gal And please donut tell yo mother, 'cause Will and Joe and several mo I know is yo half-brother."

But mama knew and said, "Honey chile, Do what makes yo happy. Marry Will or marry Joe You ain't no kin to pappy!" 


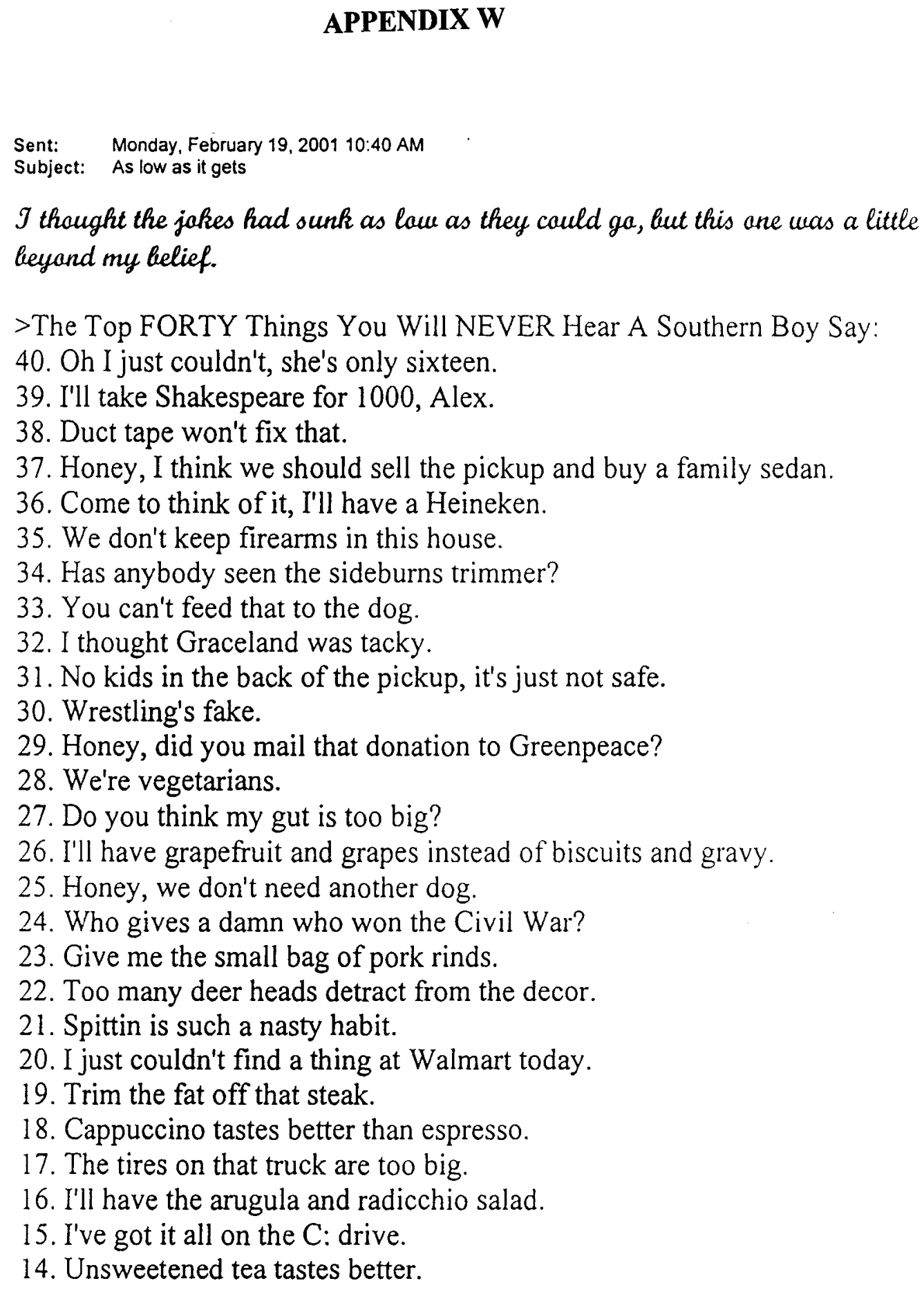

I thought the jakes had sunk as lau as they cauld ga, but this ane was a little beyand my belief.

>The Top FORTY Things You Will NEVER Hear A Southern Boy Say: 40. Oh I just couldn't, she's only sixteen.

39. I'll take Shakespeare for 1000, Alex.

38. Duct tape won't fix that.

37. Honey, I think we should sell the pickup and buy a family sedan.

36. Come to think of it, I'll have a Heineken.

35. We don't keep firearms in this house.

34. Has anybody seen the sideburns trimmer?

33. You can't feed that to the dog.

32. I thought Graceland was tacky.

31. No kids in the back of the pickup, it's just not safe.

30. Wrestling's fake.

29. Honey, did you mail that donation to Greenpeace?

28. We're vegetarians.

27. Do you think my gut is too big?

26. I'll have grapefruit and grapes instead of biscuits and gravy.

25. Honey, we don't need another dog.

24. Who gives a damn who won the Civil War?

23. Give me the small bag of pork rinds.

22. Too many deer heads detract from the decor.

21. Spittin is such a nasty habit.

20. I just couldn't find a thing at Walmart today.

19. Trim the fat off that steak.

18. Cappuccino tastes better than espresso.

17. The tires on that truck are too big.

16. I'll have the arugula and radicchio salad.

15. I've got it all on the $\mathrm{C}$ : drive.

14. Unsweetened tea tastes better. 
13. Would you like your fish poached or broiled?

12. My fiance, Bobbie Jo, is registered at Tiffany's.

11. I've got two cases of Zima for the Super Bowl.

10. Little Debbie snack cakes have too many fat grams.

9. Checkmate.

8. She's too young to be wearing a bikini.

7. Doesthe salad bar have bean sprouts?

6. Hey, here's an episode of "Hee Haw" that we haven't seen.

5. I don't have a favorite college team.

4. Be sure to bring my salad dressing on the side.

3. You All.

2. Those shorts ought to be a little longer, Darla.

And, Number ONE is:

1. Nope, no more for me. I'm drivin tonight 


\begin{abstract}
APPENDIX X
Sent: Friday, July 28, 2000 11:46 AM

Subject: Fw: Just Suppose

JUST SUPPOSE that at every ball game, graduation, prom, etc., someone who has had enough of stupid, anti-historical court decisions had the guts to start reciting the Lord's Prayer loudly, and others joined in, then more, until hundreds participated.
\end{abstract}

And JUST SUPPOSE this spread all over our land until this became standard practice in hundreds of schools-then thousands then tens of thousands.

Just what would local school boards do? Expel half the student body? (They need their jobs and federal funding far too much to do that.) What would a Federal district court do? Order hundreds of nonviolent, decent minors jailed? Or thousands?

Just what would the Supreme Court do about it - issue more edicts? What if millions decided the Supreme Court was out of its league and said "So what?"

JUST SUPPOSE hundreds of brave school board members, local judges, prosecutors, and others, each in their own area of authority, refused to intervene, realizing that we have already tried it the other way, and the result was a modern-day Sodom and Gomorra of immorality and senseless violence.

JUST SUPPOSE this then spread next into classroom after classroom, and either a brave student or a brave teacher started the Lord's Prayer (or Psalm 100, or a Bible reading, etc.) each morning, until hundreds of thousands stood up and did their part and stopped cowering before the destructive, God-hating secular attitudes of the pagan minority who parrot the media line.

JUST SUPPOSE every God-fearing Christian

(continued) 
participated-peacefully, nonviolently, but firmly and continually. Where would they get enough jails to hold us all? How would they prosecute hundreds of thousands?

It is far more than the government can do to even stop the flow of illegal drugs, despite their best efforts, and overcrowded jails. Hardened criminals walk after doing only half of their sentence to ease jail crowding.

Even if they try to pick on a handful to make examples of them, what if hundreds of thousands stood up to the tyranny of the minority and demanded their freedom of religion back? They can't build enough jails or courts to begin to deal with such a movement.

During the Civil Rights battles of the 60's, some of the black people decided they had enough and stood up to the system-a few dozen here, a few hundred there-and eventually the whole country heard, and repented, and changed. Racial repression was an evil whose time was over.

But now, there is another battle-and the stakes are even higher. The future of all children, white, black, and otherwise, is at stake. No other country on earth allows a tiny minority of impractical anti-religious bigots to censor their people's right to free religious expression. Even Russian public schools show videos of the life and teachings of Jesus now. We tend to get exactly the kind of government we deserve.

If you make a time line or a graph, you will see that the exponential increase in public school violence, pregnancy, and foul language all started at exactly the time the Supreme Court threw prayer and Bible reading out of the schools. We told God we didn't need ' Him, and the results speak for themselves.

JUST SUPPOSE hundreds of Christians passed this message on to all their friends, leaders, and contacts.

JUST SUPPOSE hundreds of churches, organizations and

(continued) 
ministries passed this message on to their constituencies with a request to reprint, re-post, and repeat it wherever possible.

Once upon a time the Church was the moral conscience and spiritual lighthouse of the nation. Now, most congregations are impotent, pusillanimous minor-league social welfare agencies or mutual comfort societies with no impact on the world around their little enclave.

JUST SUPPOSE-What do you suppose you should do?

YOU MAY NOT KNOW WHAT THE FUTURE HOLDS, BUT KNOWING WHO HOLDS THE FUTURE MAKES ALL THE DIFFERENCE. 


\title{
APPENDIX Y
}

Sent: $\quad$ Monday, August 27, 2001 7:14 PM

Subject: redneck family tree

\section{REDNECK FAMILY TREE}

\author{
Many, many years ago \\ When I was twenty three \\ I got married to a widow \\ Pretty as could be.
}

This widow had a grown up daughter

With flowing hair of red, My father fell in love with her And soon the two were wed

This made my dad my son-in-law

And changed my very life. Now my daughter was my mother, For she was my father's wife.

To complicate the matters worse Although it brought me joy, I soon became the father of a bouncing baby boy.

My little baby then became 


\section{A brother-in-law to dad, And so became my uncle, Though it made me very sad.}

For if he was my uncle, Then that also made him brother To the widow's grown up daughter, Who of course was my step-mother.

Father's wife then had a son Who kept them on the run, And he became my grandson For he was my daughter's son.

My wife is now my mother's mother And it makes me blue. Because although she is my wife, She's now my grandma too.

If my wife is my grandmother, Then I am her grandchild. And every time I think of it It simply drives me wild.

For now I have become The strangest case you ever saw, As the husband of my grandmother, I am my own grandpa! 


\section{APPENDIX $\mathrm{Z}$}

Sent: Wednesday, November 14, 2001 6:31 PM

Attach: rednec motor home.jpg; redneck high rise.jpg; Redr Swimming Pool.jpg; redneck yacht.JPG; capb.jpg Subject: FW: Redneck Luxuries

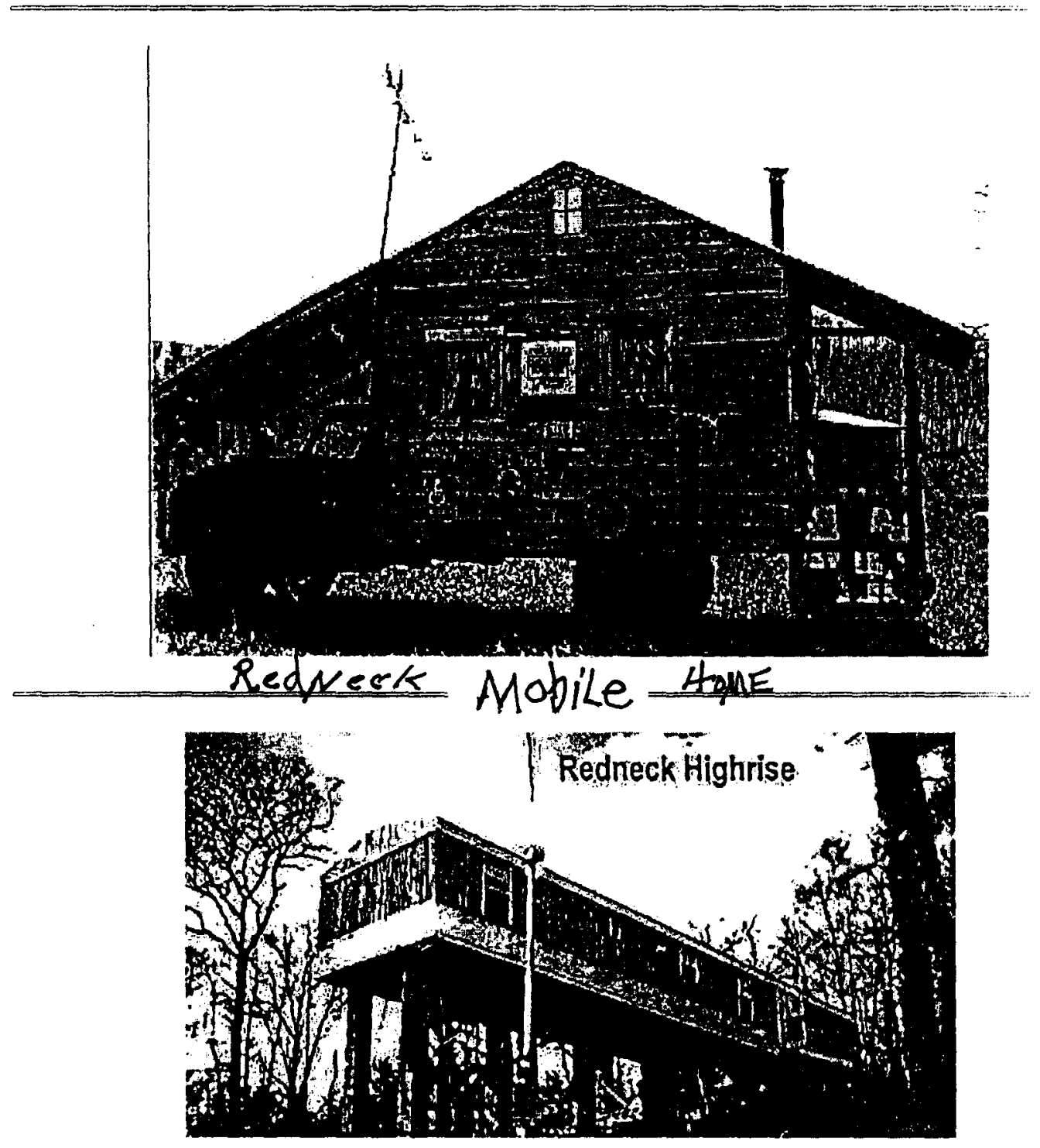

(continued) 


\section{The Pedruck Yacht}

$\div 4 y^{4}$
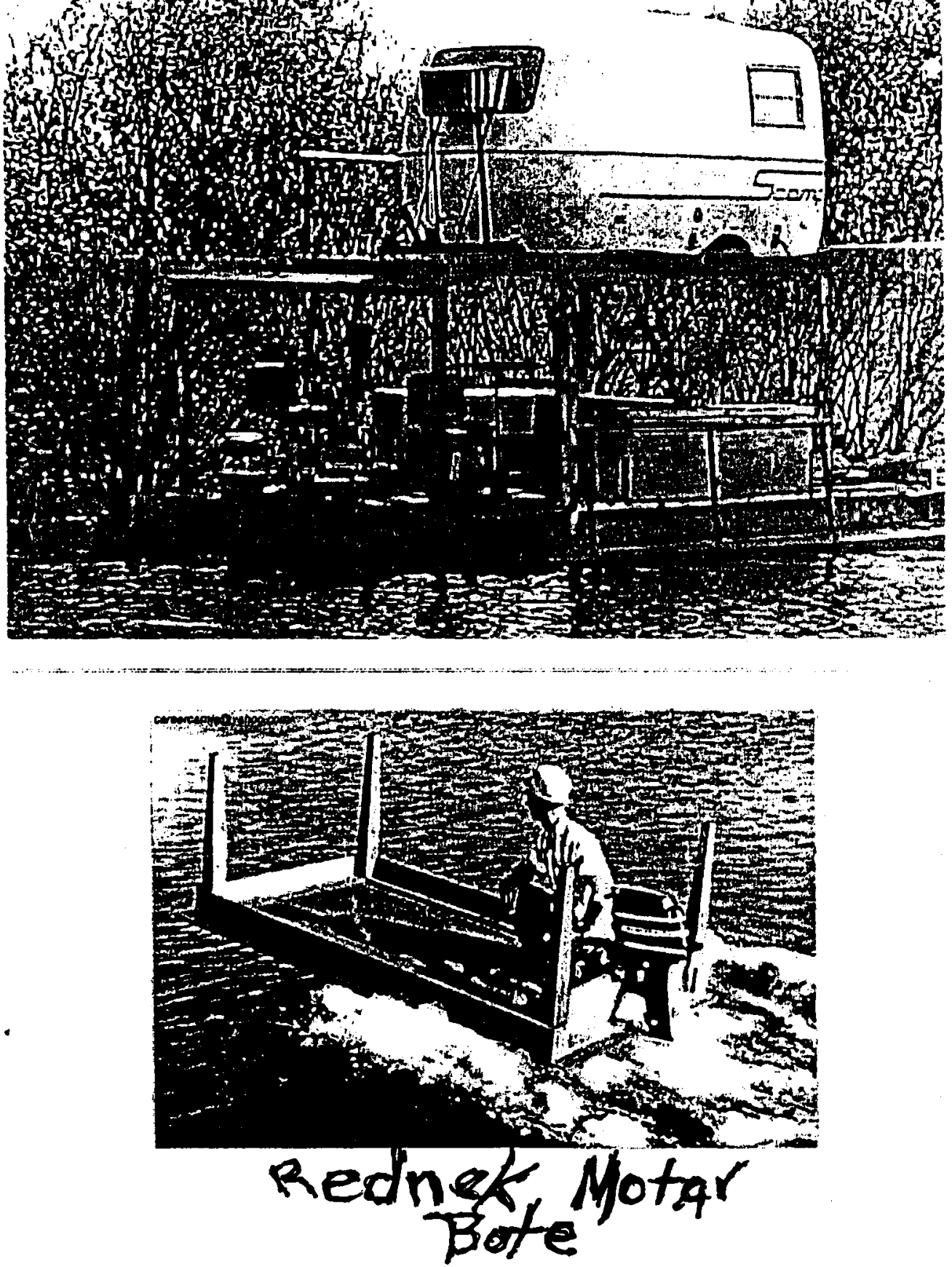


\section{APPENDIX AA}

Sent: Wednesday, June 13, 2001 6:19 PM

$>$ NOAH AND TODAY'S ARK:

$>$

$>$ The Lord spoke to Noah and said, "Noah, in six months I am going

$>$ to make it rain until the whole world is covered with water and

$>$ all the evil things are destroyed. But, I want to save a few good

$>$ people and two of every living thing on the planet. I am ordering

$>$ you to build an ark." And, in a flash of lightning, he delivered

$>$ the specifications for the ark.

$>$

$>$ "OK," Noah said, trembling with fear and fumbling with the

$>$ blueprints, "I'm your man."

$>$

$>$ "Six months and it starts to rain," thundered the Lord. "You

$>$ better have my ark completed or learn to swim for a long, long

$>$ time!"

$>$

$>$ Six months passed, the sky began to cloud up, and the rain began

$>$ to fall in torrents. The Lord looked down and saw Noah sitting in

$>$ his yard, weeping, and there was no ark.

$>$

$>$ "Noah!" shouted the Lord, "where is My ark?" A lightning bolt

$>$ crashed into the ground right beside Noah.

$>$

$>$ "Lord, please forgive me!" begged Noah. "I did my best, but there

$>$ were some big problems. First, I had to get a building permit for

$>$ the ark's construction, but your plans did not meet their code.

$>$ So, I had to hire an engineer to redo the plans, only to get into

$>$ a long argument with him about whether to include a

. > fire-sprinkler system."

$>$

$>$ "My neighbors objected, claiming that I was violating zoning

$>$ ordinances by building the ark in my front yard, so I had to get

$>$ a variance from the city planning board."

$$
>
$$


$>$ "Then, I had a big problem getting enough wood for the ark, $>$ because there was a ban on cutting trees to save the spotted owl. $>$ I tried to convince the environmentalists and the U.S. Fish and $>$ Wildlife Service that I needed the wood to save the owls, but $>$ they wouldn't let me catch them, so no owls."

$>$

$>$ "Next, I started gathering up the animals but got sued by an

$>$ animal rights group that objected to me taking along only two of $>$ each kind."

$>$ "Just when the suit got dismissed, the EPA notntied me that II

$>$ couldn't complete the ark without filing an envirommental impact

$>$ statement on your proposed flood. They didn't take kindly to the

$>$ idea that they had no jurisdiction over the conduct of a Supreme

$>$ Being."

$>$

$>$ "Then, the Corps of Engineers wanted a map of the proposed flood $>$ plan. I sent them a globe!"

$>$

$>$ "Right now, I'm still trying to resolve a complaint with the

$>$ Equal Opportunities Commission over how many minorities I'm

$>$ supposed to hire."

$>$

$>$ "The IRS has seized all my assets claiming that I am trying to

$>$ leave the country, and I just got a notice from the state that I

$>$ owe some kind of use tax. Really, I don't think I can finish the

$>$ ark in less than five years."

$>$

$>$ With that, the sky cleared, the sun began to shine, and a rainbow

$>$ arched across the sky.

$>$

$>$ Noah looked up and smiled. "You mean you are not going to destroy

$>$ the world?" he asked hopefully.

$>$

$>$ "No," said the Lord, "the government already has." 


\section{APPENDIX BB}

Life on the Farm

J. C. Wise 1929

I wrote this poem I want to say

While plowing corn the other day

Except for a little while you see

When I tarried beneath a nice shade tree

and the ice cold water was sweet I declare ..

as I lay there breathing the pure fresh air

The very air seemed filled with joy

To fire the blood of a Country Boy

The difference in the country and the city you see

Its the same as between a post and a tree

The things of your city are the products of Man

While the things of the country are made by Gods hand

Yes Boys! Life on the farm is the life for me

Where the hen lays eggs and the milk runs free

The little calf grows to be a cow, the little pig to be a sow

The fish and the rabbits are there to eat-

The blackberries grow nice and sweet

You can lay down at night on the grass nice and clean

Smoke and chew your old long green

The music of the night is the bark of a dog,

The hoot of an owl, the croak of a frog.

Boys! The things of the city have their worth-

But life on the farm is Heaven on Earth

Jack Wise - Clark County Farm Bureau

7245 Ecton Road

Winchester, Kenrucky 40391
This Poem was written by my dad more than 65 years ago. It was a big part of his heritage. It is a big part of my family heritage - our birhright. 


\section{APPENDIX CC}

\section{The ACLU Finally Meets Its Match}

\section{Legal Group Founded by James Dobson, Larry Burkett, Bill Bright, and Other Christian Leaders Emerges as a Major Contender in Contest over Values}
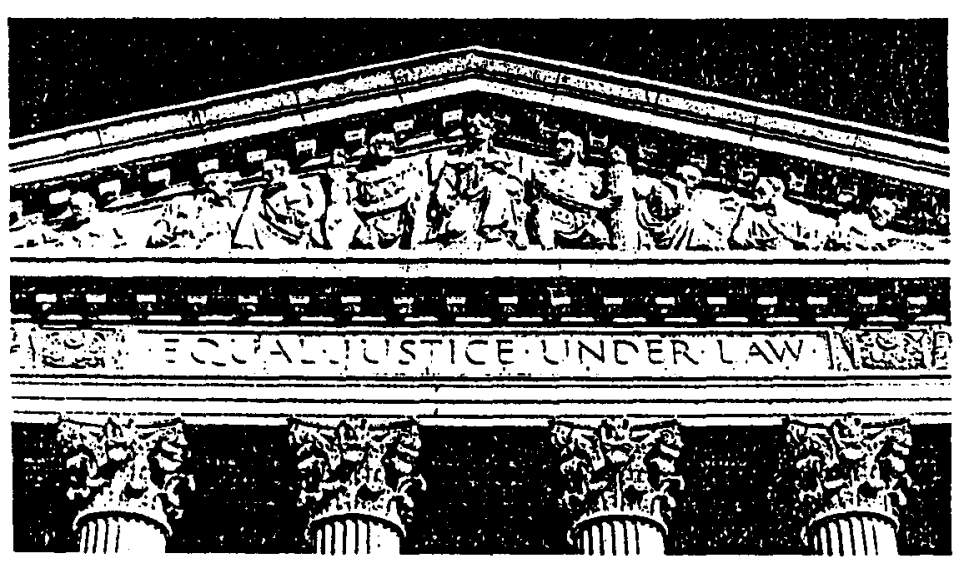

The U.S. Supreme Court has ruled on 22 major cases supported in various ways by the Alliance Defense Fund.

Tor nearly a generation, Christians have 4 laken a beating in America's legal system. 1 From the Supreme Courr's 1963 ruling that effectively banned public prayer in schools 10 its landmark Roe $v$. Wade decision that legalized aborion, Christians have soen society and is laws move sharply away from any basis in biblical principles.

From the beginning, the American Civil Liberies Union (ACLU) has led the drive lo use cour rulings to limil Christian influence in Anericin public life.

It hass' t hurn that the ACLU has been wided by hollywoud, the mainstream media, and most recently, the Clinion White House.

Where were the Christians while their rights were being taken away?

For years, they barely showed up for the batle. When the ACLU would file a suit to curb religious freedom or break some moral taboo, Christians rarely mounted a competent and effective legal challenge. As a resulh we lost. Repeatedly.

In 1994, thar picnure began to change. Top evangelical Christian leaders Bill Bright, Larry Burkert. James Dobson. D. James Kennedy, Marlin Maddoux, and Don Wildmon gathered to discuss their grave concerns over the loss of religious liberty and slipping moral standards due to negative court precedents over the preceding 30 years. They were convinced that if the trend continued, it might actually become illegal for Christians to evangelize and freely exercise their faith in America.
These leaders determined it was time for their ministries to pool their resources to begin vigorously asserting the constitutional rights of Christians.

The Alliance Defense Fund (ADF) was formed to stand against the ACLU in three critical areas: religious freedom, the sanctity of human life, and family values.

When asked why he supponed the concept of ADF, Larty Burkell, president of Christian Financial Concepts, stated, "The legal anticks on our religious treedom are escalating it an alarming rate. Through ADF, we cun unitc, pool resources, and help defend our rights."

Alan Sears. ADF's president and general counsel, explains, "We weren't formed to duplicate the work of any other group. ADFs founders wanted to provide the muscle behind these groups by developing an effective strategy, carefully selecting cases that would have the greatest long-term impact, and equipping and supporting the atomeys fighting the frondine batules in court."

He continues, "Our goal is to establish new legal precedents to reclaim the ground Christians have lost to groups like the ACLU."

It was a sensible plan. As ADF founder D. James Kennedy says, "Through ADF's crucial work, we have a fighting chance to preserve America's true heritage and value system."

7 ut in 1994, few expected that ADF would 8 have an immediate and noticeable impact. In fact, the founders anticipated years of painstaking work and some setbacks before being able to slow the ACLU juggernaut, much less stop or reverse it.

But that's not how the story has played out. In fact, ADF's presence on the legal scene began to be felt almost innediately as ADF-backed antomeys became involved in one coun victory after another.

The most notable was the U.S. Suprente Courn nuling in the case of Rusentierger to the University of Virginia.

Ron Rosenberger was a junior at the University of Virginia. A committed Christian, Rosenberger was concerned that none of the 15 student-run publications on campus offered a forum for Christian expression.

So he and some of his schoolnates decided to publish their own publication. and they applied for student activity funds.

It seemed only fair because 118 olker sludent groups-including Students for Animbal Rights, the Lesbiant and Gay Student Union, and the Muslim Students Association-aiready received school funding.

Yet Rosenberger's request for funding w'Ls denied because his group was Christian.

When he appealed to the university, Rosenberger was told that his magivine "went

"I truly believe we are fighting for the very survival of the Gospel and of evangelism in America loday!"

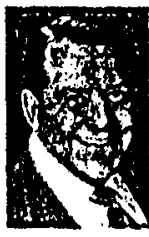

Bill Bright

over the line in saying 'we want people to live as Christians." " It was an interesting double standard because another studentfunded publication, called the Yellow Jounal, openly mocked Christianity in its pages.

Rosenberger decided to take his case to court. A group of volunteer lawyers, some of America's brightest, look his case.

Nonetheless, the judge ruled for the university on the grounds that (10tint the magazine would violate the Estublishment 
Clause of the US. Constitution; bowever, the judge gave no legal reasoning for why be believed this. After appealing the decision io the U.S. Ith Circuil Court of Appeals, the trial court ruling was affirmed.

Inter ADR. Rocenberger's lawyers spplied

$\gamma$ to the Alliance Defense Fund for $a$ Lgrant to appeal the case to the U.S. Supreme Court.

$A D F$ bas stringent standards for granting awands and a crack team of attomeys who review each case on is merits. Grants are only awanded for those cases that have the potential to establish an impartant new legal procedent. The Rosenberger case met every criteria The grant was awardod.

The US. Supreme Court agneed to hear the ADF-funded arguments and nulod S-4 in favor of the Christian students.

Writing for the majority, Justice Anthony Kennedy reasoned. "Vital First Amendment speech principles are at stake bere. [W] bold that the regulation invoked to deny fsudent fund] support, boch in ins terms and in its application to these [Christian tudents], is a denial of their right of free specech guaranteed by the First Amendment"

If was a soinning victory that put the ACLU and other liberal legal groups on notice: There's a new player on the block that can win

Alan Sears says, "Rasenberzer nox only set 2 precodent others could build on in similar cases but also establishod the ADP alliance as a major force in the battle over religious liberty and traditional values"

Sears, who was a top federal prosecutor with the US. Department of Justice before joining ADE, knows the odds apainst an upesart

\section{"The legal attacks on our religious freedom are escalating at an alarming rate. Through $A D F$, we can unite, pool resources, and help defend our rights."

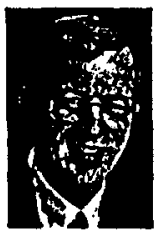 \\ Larry Burkett}

legal group having a major national impactmuch lese having the Supreme Court bear cases it belps fund.

"In a typical year, there can be more than 7,000 petitions to the US. Supreme Court"
Sears explains. "Usually fewer than 150 are acoepted for full review. That makes the odds of having a case beard about 46:1, 10 say nothing of winning"

"I urge every Christian to stand with me in support of $A D F$ 's critical case-funding work Already, $A D F$ is having a powerful impact in the nation's courts."

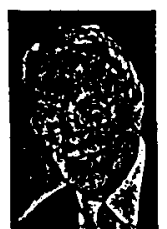

Dr. James Dobson

And yet, since 1994, the Alliance Defense Fund bas supported in various ways 22 Supreme Court cases. Remarkably, the court's action in 16 of those cases resulted in victories for religious freedom, family values, or the sanctity of life.

At the same time, ADF has been involved in nearly 130 precedent-setting lower court victories.

Each of these nulings becomes in important part of case law that is used by attomeys in arguing future, similar cases. The more positive nulings in the areas of ADFe concem, the better.

"Each new favorable nuling is like a brick" explains Sears. "One by one you put them in plece. Over the years we bope to erect a wall of precedent that can't easily be breached by those who oppose Christian values."

It's the eame strategic approach, but with far different objectives, used by the ACLU to wall of Christian influence from society. For ADF, it's a strategy that's working.

But the effect of ADFs wark is also felt outside the courtroom. New, hard-woa precedents are being used to stop the violation of Christians' rights without resarting to costly litigation.

Foc example, a group of homosexuals in Florida planned to force its way into a parade sponsored by a group of Crristian ministers.

The pastors pointed out that the Supreme Court's 90 decision in an ADR becked case, Hurley $x$ Irishnmerican Cax Lesbian and Bisexual Group of Barton prohibiled forced speoch and expression in the private aress. The bomosexuals, sware that they would lose in court, backed off, and the Christians' parade went forward undisturbed.
"Without the Hurley precedent," says Sears, "those Christians in Florida either would have bad to let the homosexuals in or cancel their parade. Had that happened, bomosexual sctivists would have been emboldened to shut down any public gathering of Christians they wanted across the country."

Despite impressive victories like Rasenberger and Hurloy, bowever, Sears is cancerned about the future.

"There are some major threats to the family that aren't going away soon" says Sears. "In fact, they're likely to get worse. We've gol a loc of wark to do."

He refers to effarts by homosexual activists to use the courts and legal precedent to redefine the family in their pursuit of legalized same-sex "mariage" and ocher special privileges.

"If the homosexuals achieve their goals, you and I won'I recognize America" explains Searc. "The homosexusal sqenda affects every ares of ADF's miscion-religious freedom. family values, and the sanctity of life. Thar's why $A D F$ is committed to fighting it tooth and nsil."

$A D F$ has already funded 42 family redefinition cases and beld a National Litigation Academy, Defining the American Fanily and Its Legal Future.

Mare than 40 Christian attorneys received advanced legal training that they can use in the courtroom to defeat bomosexual activists who want to redefine and destroy the family.

"ADF has proven it has the right stralegy 10 attack this problem," says Sears "What we need are more financial resources to wage a nuccessful Gight against the homocexual agenda. I bope that as Christians hear abour ADF and recognize the strategic importance of our wark, they will give to help us. The future of our country and our frodoms as Christians are at stake"

Sears pauses before adding "These battles will be waged in the nation's courts whether or nox Christians show up to fight We lost by defaule for 30 years. Now, with ADP on the scese, it is our goal not to let that happen again" AP was established as a nonprofit organization funded entirely by the taxdeductible contributions of people who share the vision of ADF's founders

It also establisbed a firm no-debt policy. ADP only financially backs new cases and invests in training and straticgic planning as funds are available. And according to Sears, more funds are urgently needed-especially to fight the rapidly escalating bomosexual threat in courtrooms across the country

To make a contribution to the Alliance Defense Pund, chocks can be sent to ADF, 7819 E Greenway Road, Suive 8, Scottsdale. AZ 85260. 


\section{CURRICULUM VITAE}

NAME: E Carolyn Tucker
ADDRESS:
429 Burnt Mill Road
Dixon, KY 42409
DOB: $\quad$ Henderson, Kentucky - May 2, 1949

\section{EDUCATION}

\& TRAINING:

$1967-1972$

B.S., Political Science, Geography, English, Secondary Ed.

$1978-1980$ Murray State University, Murray, Kentucky

$1993-1995$

M.A., Secondary Education/English

$1992-1994$

Murray State University, Murray, Kentucky

1995

1994, 1998

1994

Kentucky Distinguished Educator

$1990-1991$

Kentucky Education Reform Act Fellow

Regional Service Center Associate

Kentucky Teacher Intern Program (KTIP)

Partnership Missionary to Russia

Assistant Director of Purchase Area Writing Project (PAWP)

AWARDS: $\quad$ Who's Who In American Education 1989

Who's Who in the South and Southwest 1991, 1992, 1995

Who's Who Among American Teachers 1990, 1992, 1994, 2004

International Who's Who 1993

Who's Who in the World 1994

Kentucky Distinguished Educator

PROFESSIONAL SOCIETIES: Association for the Sociology of Religion (ASR)

National Association of Secondary School Principals (NASSP)

Phi Delta Kappa (PDK)

Association for Supervision and Curriculum Development (ASCD)

National Rural Education Association (NREA)

Society for the Scientific Study of Religion (SSSR)

Webster County Retired Teachers Association

Kentucky Retired Teachers Association

American Association of Religion (AAR)

PUBLISHED IN:

Un-covering the Curriculum

Event

Encounter

Contempo

Kentucky English Bulletin

The Link

Council on English Leadership

Essential Connection 